\title{
Recrutement et consentement à la recherche : réalités et défis éthiques
}

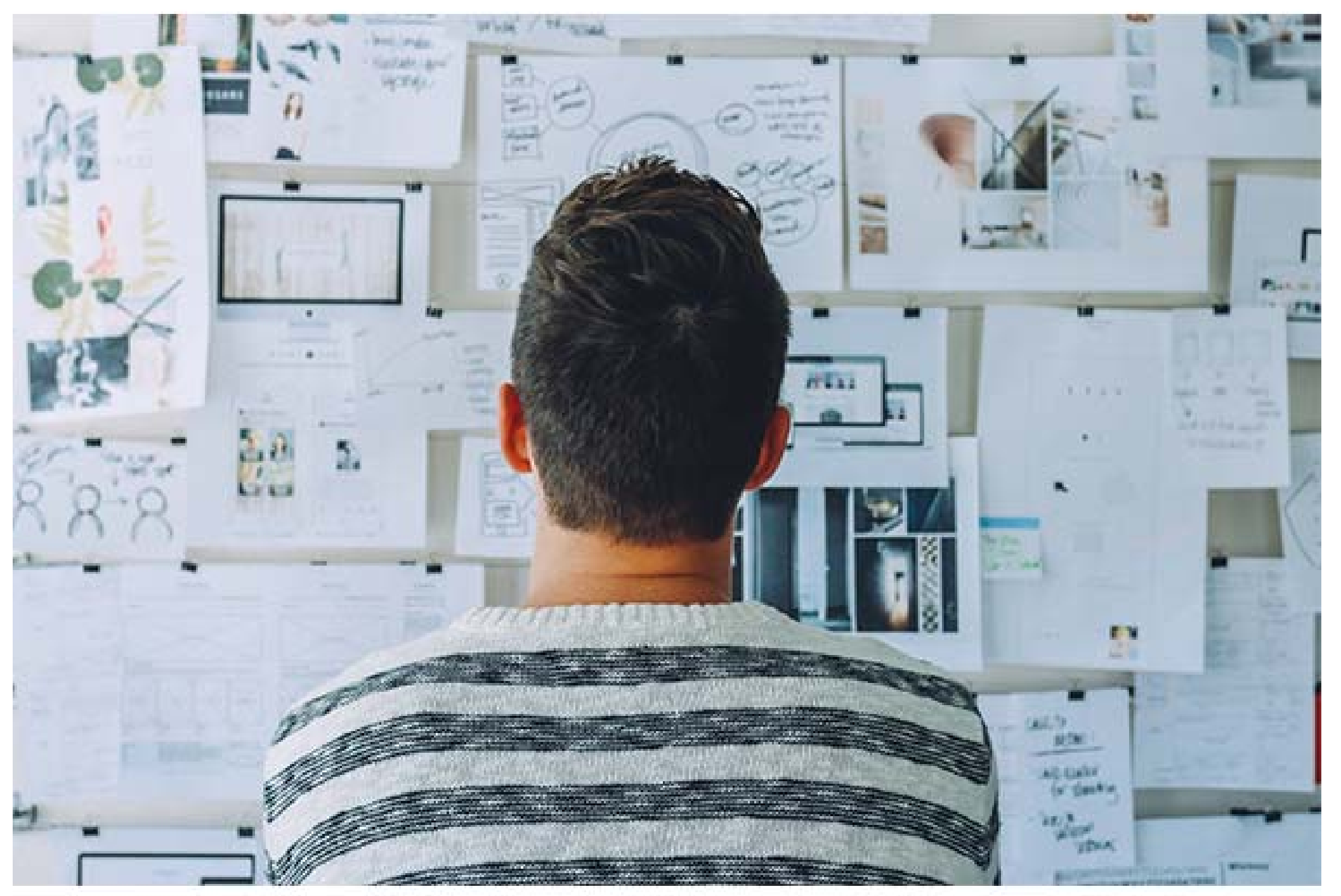

Sous la direction de Ana Marin, Béatrice Eysermann et Michel T. Giroux 
PHOTO PAR : STARTUP STOCK PHOTOS VIA LE SITE WWW.PEXELS.COM

SOURCE : HTTPS://WWW.PEXELS.COM/PHOTO/MAN-WEARING-BLACK-AND-WHITE-STRIPE-SHIRTLOOKING-AT-WHITE-PRINTER-PAPERS-ON-THE-WALL-212286/

Licence CReative Commons : CCO 
Publication: ReCRUtemEnt ET CONSENTEMENT À LA RECHERCHE

PAGES: $1-209$

ISBN: 978-2-7622-0357-8

Directeurs: AnA MARIN, BÉATrice Eysermann et Michel T. GIROUX

URI: HTTP://HDL.HANDLE.NET/11143/14103

DOI: HTTPS://DOI.ORG/10.17118/11143/14103 


\section{Remerciements}

Nous tenons à remercier pour leur contribution financière:

- les Instituts de recherche en santé du Canada (IRSC, \#142187),

- le CHU de Québec - Université Laval,

- la Direction de la recherche du CISSS de Chaudière-Appalaches

- le CISSS du Bas-Saint-Laurent

- la Direction de la recherche du CIUSSS de la Capitale Nationale

Nous remercions Chantal Bouffard pour son appui et sa confiance pour l'organisation du colloque «Consentez-vous (toujours) à participer à ma recherche? » Processus de demande et de renouvellement de consentement/d'assentiment à des personnes vulnérables» (Colloque 29, Acfas 2015).

Nous remercions aussi l'Acfas pour nous permettre d'organiser des activités pluridisciplinaires, tels nos deux colloques qui ont été à la source de ce collectif.

Et finalement, un grand merci à Jonathan Mc Lellan pour son travail de révision et d'édition des textes publiés dans cet ouvrage. 


\section{Table des matières}

Remerciements

Ana Marin, Béatrice Eysermann et Michel T. Giroux

Introduction générale

Ana Marin, Béatrice Eysermann et Michel T. Giroux

Objectivité et bienveillance du chercheur à l'égard du sujet de recherche 8

Michel T. Giroux

Littératie et consentement éclairé à la recherche : état des lieux et pistes de solution. 28

Ana Marin et Michel T. Giroux

Les projets cliniques à la frontière de la recherche expérimentale et du traitement innovateur:

Quel consentement?

Johane Patenaude

L'incertitude au cœur de la tension éthique du recrutement effectué

par le clinicien-chercheur

Bruno Leclerc et Alexandra Dubé-Loubert

Recrutement en phase 1 chez des participants atteints d'une maladie grave 66

François Pouliot

À quoi une personne souffrant d'un trouble mental consent-elle dans un contexte de recherche?

Jacques Quintin

Recruter des «travailleurs de l'ombre» : le défi de la participation

chez les préposés aux bénéficiaires

Émilie Allaire et Nathalie Jauvin

Comprendre la marginalité ou renforcer la marginalisation?

Réflexions éthiques autour d'une recherche.

Éric Gagnon

Recherche sur la spiritualité : les défis du recrutement

Bruno Bélanger et Line Beauregard

Les problématiques du recrutement auprès des patients atteints de traumatismes craniocérébraux (TCC) et d'Alzheimer.

Kim Charest et Julie Bouchard 
«Veux-tu participer à ma recherche?» : principes, enjeux et stratégies concernant l'assentiment des enfants dans le processus de recherche.

Isabel Côté, Renée-Pier Trottier Cyr, Kévin Lavoie et Geneviève Pagé

Relation de confiance, un ancrage incontournable en contexte de recherche autochtone: témoignages de recrutement de participants atikamekws, inuit, anicinapek et innus en éducation

Yvonne da Silveira, Elisabeth Jacob, Glorya Pellerin et Véronique Paul

Navigateurs, milieu de la finance et bénévolat : souvenirs de terrain et réflexions transversales sur le recrutement.

Béatrice Eysermann

Analyse rétrospective de l'utilisation des réseaux sociaux pour le recrutement d'adolescent(e)s atteints de NF1, de femmes enceintes, de chercheurs/euses fondamentalistes et de médecins. 178

Frédérique Duplain-Laferrière, Gabrielle Lapointe, Véronique Noël, Gaël Bouffard, Chantal Bouffard

Conclusion .204

Béatrice Eysermann, Michel T. Giroux et Ana Marin 
TITRE: INTRODUCTION GÉNÉRALE

Auteur(s): Ana Marin, Béatrice Eysermann et Michel T. Giroux

Publication: RECRUTEMENT ET CONSENTEMENT À LA RECHERCHE : RÉALITÉS ET DÉFIS ÉTHIQUES

PAgES: $1-6$

ISBN: 978-2-7622-0357-8

Directeurs: ANA MARIN, BÉATRICE EYSERMANN ET MICHEL T. GIROUX 


\section{Introduction générale}

Ana Marin, professeure associée Université de Sherbrooke, membre des Comités d'éthique de la recherche du CHU de QuébecUniversité Laval et du CISSS Chaudière-Appalaches, conseillère en éthique CISSS Chaudière-Appalaches, membre de l'Institut de consultation et de recherche en éthique et en droit (ICRED) et membre associée de l'IDEA.

Béatrice Eysermann, Ph. D., coordonnatrice du Comité d'éthique de la recherche sectoriel en santé des populations et première ligne, CIUSSS de la Capitale-Nationale.

Michel T. Giroux, avocat et docteur en philosophie, directeur de l'Institut de consultation et de recherche en éthique et en droit (ICRED), président du Comité d'éthique de la recherche des établissements du Centre de recherche interdisciplinaire en réadaptation du Montréal métropolitain (CRIR), président du Comité d'éthique de la recherche Biobanque Genizon chez Génome Québec, professeur associé au département de médecine sociale et préventive à la Faculté de médecine de l'Université Laval.

L'éthique de la recherche reste encore un domaine peu propice à la réflexion éthique des principaux concernés, à savoir les chercheurs, leurs équipes et les étudiants. Et ce, pour deux raisons. La première : L'éthique de la recherche reste dans bien des cas une chasse gardée des comités d'éthique de la recherche (CER), de par leur mandat et leur fonctionnement, certes, mais aussi de par la chronologie d'un projet de recherche en lui-même; les chercheurs préparent leurs projets et, ensuite, les soumettent au CER pour approbation éthique. On peut alors déjà se questionner sur la teneur de la formation en éthique et sur la réflexion éthique suscitée chez les chercheurs rendus à cette étape. La deuxième raison est liée à la publication scientifique : Si les chercheurs se sentent encouragés à publier des résultats de recherche, ce n’est guère le cas lorsque cela concerne la réflexion éthique des étapes de leurs recherches sur le terrain. Autrement dit, si on trouve si peu de publications sur les enjeux éthiques du recrutement ou du processus de demande de consentement à la recherche, on peut en déduire que ce champ est moins prisé ou moins essentiel aux yeux tant des chercheurs que des revues scientifiques.

Face à ce manque d'espace de réflexion éthique partagé avec ceux qui font de la recherche, il nous est venu l'idée d'organiser une rencontre sous forme de colloque dans le cadre du Congrès annuel de l'Acfas 2015'. Le sujet nous est venu spontanément à l'esprit, puisqu'il est au centre de plusieurs discussions en au sein des CER, à savoir : le consentement - ou, plus précisément, les processus de demande et de renouvellement du consentement et de l'assentiment - dans les projets de recherche avec des personnes vulnérables, aptes ou inaptes, ou avec des enfants. La vulnérabilité a été ici prise au sens large. Il s’agit alors de personnes éprouvées de diverses façons, atteintes ou non de maladie, mais souffrant dans leur vie personnelle et dans leur environnement social. Nous avons ciblé les personnes vulnérables, car nous savions que l'étape de la demande du consentement serait aussi cruciale que délicate. Participer à un projet de recherche implique préalablement de prendre conscience des critères de sélection de ladite recherche, ce qui peut mener à une prise de conscience de sa propre situation : violence conjugale, fragilité, maladie, etc. C'est un premier dévoilement avant de donner son consentement pour en dire davantage, pour révéler des détails sur les aspects les plus difficiles ou intimes de sa vie, ou pour livrer son corps pour l'avancement de la recherche.

1. «Consentez-vous (toujours) à participer à ma recherche? » Processus de demande et de renouvellement de consentement/ d'assentiment à des personnes vulnérables (http://www.acfas.ca/evenements/congres/programme/83/enjeux-recherche/29/c) 
Notre objectifétait de mettre de l'avant ce qui se passe sur le terrain lors du processus de demande de consentement ou d'assentiment, et lors du renouvellement de ces derniers. Que ce soit lors d'essais cliniques ou de recherche à caractère plus social, le consentement, tout en étant la pierre angulaire de la participation du sujet au projet de recherche, peut représenter un véritable défi. Si l'éthique de la recherche a créé un outil sous forme de formulaire de consentement - lequel ne cesse de soulever les passions sur sa véritable vocation - il n'est bien souvent que la dernière étape à franchir avant la collecte des données. Et, comme il existe bien des façons de demander ce consentement, notre objectif n'était pas de débattre des controverses concernant les seuls formulaires de consentement; ce qui nous intéressait, c'étaient les réflexions autour du processus qui conduit au consentement. Entre autres, comment s'y prend-on face à des personnes vulnérables? Combien de temps prend-on? Est-ce délicat, difficile, intimidant pour les parties impliquées? Comment s'assure-t-on de leur consentement tout au long de la recherche? Quels en sont les défis, les ratés, les succès? Ultimement, l'objectif du colloque était de partager les expériences vécues sur le terrain et d'échanger sur les obstacles et les pistes de solutions possibles.

Ce colloque, à notre grande et agréable surprise, a eu un succès remarquable, démontrant que ce type d'activité répondait effectivement à un besoin pour les protagonistes de la recherche. Ils ont répondu à l'appel, ont participé activement au débat et ont montré leur intérêt pour une autre activité de ce genre. L'année suivante, nous récidivions avec un deuxième colloque 2 . Cette fois, le but était de se pencher sur le recrutement des participants à la recherche, un processus délicat à la genèse de tout projet de recherche. Le recrutement était défini dans ce contexte comme un processus qui va de pair avec le consentement et qui vise à approcher les sujets potentiels afin de susciter leur intérêt pour une recherche.

On s'est ainsi proposé de débattre du processus de recrutement des participants à la recherche, de ses enjeux éthiques, des défis rencontrés et des stratégies développées par les chercheurs et leurs équipes sur leur terrain de recherche. Le recrutement soulève beaucoup de questions éthiques, scientifiques et pratiques. Les balises éthiques qu'on se donne reposent notamment sur les principes de respect de la personne, de bienveillance et de justice... Mais, autour de la nature et du contexte spécifique de chaque projet de recherche, comment cela s'articule-t-il? Et autour de la vulnérabilité de la population à l'étude et du lien que le chercheur entretient avec les participants potentiels? Pourquoi les patients hospitalisés en CHSLD ou les proches aidants semblent-ils plus difficiles à approcher? Sans s'y restreindre, fait-on le même constat en contexte de maltraitance, de violence conjugale et lorsqu'il s'agit de mineurs ou de personnes marginalisées? La pratique largement répandue et «éthiquement correcte» pour les CER de placer systématiquement un intervenant comme intermédiaire entre le chercheur et le participant potentiel est-elle vraiment la meilleure approche de recrutement? Quelles stratégies fonctionneraient au mieux et dans quels contextes? À l'heure du 2.0, le recrutement en ligne serait-il une solution? Quels sont les enjeux liés à l'approche directe des personnes? Comment faire face aux pressions externes des patients et des promoteurs, notamment dans le cas des essais cliniques? Ces réalités de terrain où s'entremêlent pouvoir, biais de désirabilité ou concurrence pourraient-elles aller jusqu'à compromettre la validité scientifique des données recueillies? Autant de questions qui, et ce fut notre objectif, permettraient de débattre des enjeux du recrutement tels qu'ils sont vécus sur le terrain. Nous y tenions.

2. "Seriez-vous intéressé(e) par mon projet de recherche? » Sortir des sentiers battus : les défis du recrutement à la recherche, (http://www.acfas.ca/evenements/congres/programme/84/enjeux-recherche/13/c) 
Cette fois encore, nous avons invité les chercheurs, leurs équipes et les étudiants à contribuer à la réflexion dans le but de mieux cerner les défis du recrutement des participants à la recherche. Nous espérions en apprendre davantage en mettant en évidence les expériences, les écueils, les stratégies, les bons coups, les doutes, bref, tout ce qui permettrait d'amorcer des débats sur cette dimension de la recherche qui, selon notre constat, n'était que très peu discutée. Nous espérions pouvoir alors nous glisser hors des sentiers battus pour discuter des enjeux de ce processus. À noter que le politiquement correct n'avait pas sa place dans nos échanges, car nous voulions entendre parler le terrain.

Devant l'intérêt manifesté pour ces deux activités, l'idée de la diffusion du fruit de ces travaux est survenue tout naturellement, comme une évidence. La plupart des conférenciers ont répondu à l'appel et c'est ce qui a donné corps à cet ouvrage, qui présente des textes inédits, exclusivement ancrés dans la pratique sur le terrain.

L'ouvrage débute par l'article de Michel T. Giroux, qui introduit la réflexion éthique sur le recrutement et le consentement. Partant de trois événements réels en recherche, l'auteur fait ressortir les éléments suivants : l'énergie du désespoir, qui incite nombre de personnes à participer à des projets de recherche; la difficile distinction entre les finalités thérapeutiques et celles de la recherche; et, enfin, la méprise thérapeutique qui s'ensuit - tous des éléments qui reviennent dans d'autres articles du présent collectif. Deux qualités du chercheur et de son équipe contribuent, selon l'auteur, à la possibilité, pour le sujet potentiel, de participer à la recherche en exprimant une volonté libre et éclairée : l'objectivité et la bienveillance. L'objectivité est présentée ici comme une préoccupation du chercheur de faire abstraction de ses intérêts et de ses opinions personnelles. La bienveillance représente l'attitude de la personne qui montre de bonnes dispositions à l'égard de quelqu'un. La nature éclairée du consentement est ensuite interrogée, par Ana Marin et Michel T. Giroux, en lien avec le bas niveau de littératie en santé pour la majorité de la population, une difficulté à laquelle s'ajoute celle du niveau de complexité de la recherche. Partant de la définition de la littératie en santé issue des travaux de l'ICRED ${ }^{3}$ et d'une revue de littérature sur la compréhension des sujets à la recherche, les auteurs proposent une réflexion sur les principes éthiques en jeu : l'autonomie et la justice. Des pistes de solution sont ensuite proposées pour améliorer le caractère éclairé du consentement.

Les articles qui suivent abordent des enjeux éthiques en lien avec les processus de recrutement et de consentement dans des contextes particuliers utilisant diverses méthodes auprès de populations différentes. Si les auteurs des premiers articles nous amènent dans le domaine de la recherche en santé, la recherche en sciences de l'éducation et en sciences sociales a aussi une grande part dans ce recueil, permettant ainsi une contribution tout à fait originale et innovante.

Ainsi, Bruno Leclerc et Alexandra Dubé-Loubert se questionnent sur la place de l'incertitude clinique dans le processus de recrutement lorsque le chercheur est aussi le médecin traitant du participant potentiel à la recherche. La relation entre la clinique et la recherche clinique mérite réflexion. En effet, quel type de consentement doit-on privilégier lorsqu'une activité médicale standard devient un traitement novateur ou, même, une expérimentation? Johanne Patenaude traite de ce sujet dans son article, notamment, dans un contexte où le concept de traitement novateur est disparu du Code civil lors de sa dernière modification de 2013. L'auteure fait ressortir quelques lieux de confusion quant à l'opérationnalisation en recherche et en clinique de ce concept et propose quelques pistes de solution pour améliorer la qualité du consentement. François Pouliot examine quant à lui les enjeux éthiques du consentement dans le contexte des essais cliniques de phase 1 avec, comme recrues, de plus en plus de personnes atteintes, contrairement à un essai de phase 1 classique, qui s'effectue davantage avec la collaboration de personnes saines. Voilà quelques-unes des pistes explorées

3. Institut de consultation et de recherche en éthique et en droit 
par ces auteurs dans le volet de recherche clinique de ce collectif. La nature du consentement, questionnée par François Pouliot dans les recherches de phase 1, on la retrouve ensuite dans la réflexion de Jacques Quintin, qui amène le lecteur dans le champ de la recherche en santé mentale. Ce dernier montre que la personne appuie sa décision sur ce qui fait sens pour elle et que le sens émerge du dialogue. D'où la démarche dialogique proposée par l'auteur comme moyen de répondre aux défis du processus de consentement des personnes atteintes de problèmes de santé mentale.

Mais la vulnérabilité des sujets potentiels est aussi réfléchie sous l'angle de la marginalisation. Comment faiton pour s'adresser à des personnes qui sont marginalisées, soit dans l'espace public, comme le met en lumière Éric Gagnon, soit dans un système professionnel, comme c'est le cas des préposés aux bénéficiaires présentés par Émilie Allaire et Nathalie Jauvin? Ces auteurs contribuent à la réflexion sur le recrutement en nous amenant vers la signification du regard que le chercheur porte sur les membres de la population à l'étude, et réciproquement sur le regard que ces personnes ont sur elles-mêmes. Comment faire pour que la recherche ne stigmatise pas davantage les personnes déjà marginalisées par la société, comme les sans-abri, les prostituées, etc. ? Car l'utilisation de certains mots et le sens que les participants leur donnent peuvent jouer des tours aux chercheurs, comme le présentent Bruno Bélanger et Line Beauregard. Faisant alors une recherche sur la spiritualité - recherche pour laquelle le CER a exigé de faire passer le recrutement par un intermédiaire, ici un intervenant - les auteurs se sont vite rendu compte que leurs pivots de recrutement ne présentaient la recherche qu'aux personnes qui avaient chez elles des items religieux, notamment chrétiens, ce qui posait en soi un biais important dans l'échantillonnage désiré. En effet, cette présélection pouvait nuire à la recherche, car elle maintenait l'association culturellement admise entre la spiritualité et la présence de signes religieux aux dépens de toute autre forme de spiritualité.

On peut ainsi se poser la question de la nécessité ou du bien-fondé de la sollicitation d'un intermédiaire, surtout lorsque le recrutement semble poser encore plus de défis, comme c'est le cas pour les populations souffrant de TCC ou d'Alzheimer ou bien encore avec les enfants, comme en témoignent le texte de Kim Charest et coll. de même que celui d'Isabelle Côté et coll. Comment approcher ces populations? Quel langage utiliser pour bien se faire comprendre? Quelles sont les barrières et les pistes à envisager? En réponse à ces questionnements, le collectif met en lumière la créativité des chercheurs sur leur terrain, en présence de leurs participants potentiels. En étant attentifs aux défis que pose la participation à la recherche pour les patients souffrant de TCC ou d'Alzheimer, Kim Charest et coll. proposent des ajustements pour bien se faire comprendre par les participants potentiels, pour les rassurer et pour leur faciliter la tâche (par l'éducation à la technologie, par exemple). On note aussi la stratégie utilisée par l'équipe d'Isabelle Côté, qui met en place toute une méthodologie de co-construction d'une Lettre de permission, alias un formulaire d'assentiment, directement avec les enfants participants, dans un souci évident du respect de la volonté, et de l'imagination, de ces derniers.

Les auteurs des trois derniers textes nous amènent sur le terrain de la recherche qualitative - en éducation, en santé et en anthropologie - auprès de populations pour le moins très différentes : des autochtones pour Da Silveira et coll. ; des navigateurs, de grands financiers et des bénévoles chez Béatrice Eysermann; et des adolescents atteints de NF1, de femmes enceintes, de chercheur(euse)s fondamentalistes et de médecins, pour Frédérique Duplain-Laferrière et coll. Le recrutement s'est fait directement sur le terrain dans les deux premiers textes, et par le biais de réseaux sociaux dans le troisième. La recherche en contexte autochtone, sous forme, ici, de recherche-action ou de recherche collaborative, répondait à un besoin d'établir un certain équilibre entre les chercheurs et les représentants de ces nations. Si la confiance a une importance significative dans la recherche en général, elle devient une véritable nécessité dans ce contexte où les rapports avec les blancs sont fragilisés par l'histoire du Canada et des Peuples autochtones. Établir, et maintenir, le lien de confiance, 
c'est ce qui permet à ce type de recherche de pouvoir ultimement répondre aux besoins de ces populations. Da Silveira et coll. illustrent ces enjeux en partant de trois projets de recherche en éducation réalisés chez des Atikamekws, des Inuit et des Anicinapek et Innus. Réfléchissant sur son thème fétiche du temps et cherchant à comprendre le temps social dominant de sa société, Béatrice Eysermann nous fait plonger dans le processus de recrutement de participants issus de trois univers complètement différents : celui des navigateurs solitaires, celui des grands financiers et enfin celui des bénévoles d'une association caritative. Elle met ainsi en lumière tant la nécessité de connaître son terrain que l'aspect caméléon du chercheur pour parvenir à approcher ses participants et à les faire adhérer à la recherche. On retrouve par là même la notion qu'a évoquée Michel T. Giroux lors du colloque, à savoir celle, peu flatteuse, peut-être, mais ô combien vraie, de démon séducteur.

Enfin, les limites du recrutement classique ont amené Frédérique Duplain-Laferrière et coll. vers le recrutement et le consentement en ligne, faisant appel pour cela à des réseaux sociaux comme Facebook, Linkedın, ResearchGate. Partant de trois projets de recherche, l'équipe décortique les dimensions éthiques du recrutement au sein des réseaux sociaux. Les auteures en illustrent les défis d'équité, de représentativité et d'accès aux bénéfices de recherche, de même que les défis de confidentialité - si chère à tous les chercheurs - et de protection de la vie privée, et ce, dans le respect de la personne et de l'intégrité de ses identités virtuelles. Par ailleurs, elles soulèvent l'importance d'une approche collaborative et co-constructive entre les chercheurs et les comités d'éthique de la recherche.

Nous pensons que ce collectif va contribuer de manière significative à la recherche sur le terrain et qu'il représente un espace de réflexion fertile pour les recherches présentes et futures. Nous espérons que vous aurez autant de plaisir à lire les recherches et découvertes des auteurs que nous en avons eus à les entendre lors de nos deux colloques.

Bonne lecture!

Ana, Béatrice, Michel 
TITRE: OBJeCtIVITÉ ET BIENVEILLANCE DU CHERCHEUR À L'ÉGARD DU SUJET DE RECHERCHE Auteur(s): Michel T. GIROUX

Publication: ReCRUtement et CONSENTEMENT À LA RECHERCHE : RÉALITÉS ET DÉFIS ÉTHIQUeS

PAGES: $7-26$

ISBN: 978-2-7622-0357-8

Directeurs: AnA MARIN, BÉATRICE EYSERMANN ET MICHEL T. GIROUX

URI: HTTP://HDL.HANDLE.NET/11143/14104

DOI: HTTPS://DOI.ORG/10.17118/11143/14104 


\section{Objectivité et bienveillance du chercheur à l'égard du sujet de recherche}

Michel T. Giroux, avocat et docteur en philosophie, directeur de l'Institut de consultation et de recherche en éthique et en droit (ICRED), président du Comité d'éthique de la recherche des établissements du Centre de recherche interdisciplinaire en réadaptation du Montréal métropolitain (CRIR), président du Comité d'éthique de la recherche Biobanque Genizon chez Génome Québec, professeur associé au département de médecine sociale et préventive à la Faculté de médecine de l'Université Laval.

Résumé : Celui qui accepte de contribuer à un projet de recherche est fréquemment appelé participant. À partir d'une comparaison entre les mots participant et sujet, voyons lequel désigne le plus adéquatement la personne qui contribue à un projet de recherche. Examinons aussi lequel de ces termes est le plus susceptible d'inspirer au chercheur le respect à l'égard de la personne qui contribue à son projet de recherche. L'enfance et l'inaptitude sont deux conditions inexorables de vulnérabilité. II faut y ajouter le désespoir, qui compromet l'aptitude à décider de façon libre et éclairée. Le phénomène de la méprise thérapeutique résulte d'une incompréhension de la différence entre la finalité de la recherche et la finalité de la clinique. Le chercheur soucieux de recruter des sujets qui se représentent adéquatement son projet de recherche, et qui y consentent véritablement devrait adopter deux attitudes à leur égard : l'objectivité et la bienveillance.

Mots-clés : participant, sujet, méprise thérapeutique, objectivité, bienveillance.

Abstract: The one who agrees to contribute to a research project is frequently called participant. From a comparison between the words participant and subject, let us see which one designates most adequately the person who contributes to a research project. Also let us examine which of these words is the most susceptible of inspiring in the researcher respect towards the person who contributes to his research project. Childhood and inaptitude are two inexorable conditions of vulnerability. It is necessary to add despair, which compromises the capacity to decide in a free and enlightened way. The phenomenon of therapeutic misconception results from the incomprehension of the difference between the purpose of research and the purpose of clinical treatment. The researcher worried of recruiting subjects who understand his research project adequately, and which really agree to it should adopt two attitudes towards them: objectivity and benevolence.

Keywords: participant, subject, therapeutic misconception, objectivity, benevolence. 


\section{Introduction}

La contribution d'un sujet à un projet de recherche peut être motivée par les raisons les plus diverses, qu'elles soient réalistes ou chimériques: contribuer au progrès scientifique, obtenir une attention médicale particulière, profiter d'une meilleure chance de guérison, s'arracher à la détresse, renouveler une espérance amoindrie, rencontrer de nouvelles personnes, se sentir utile à ses concitoyens, etc. De son côté, le chercheur a besoin de personnes qui contribuent à son projet. Ses objectifs peuvent être aussi divers que ceux du sujet. Le microcosme que constitue l'univers de la recherche en santé illustre exactement la nature sociale de l'être humain, en ce que chacun a besoin d'autrui pour se réaliser ${ }^{1}$.

Celui qui accepte de contribuer à un projet de recherche en y investissant sa personne est fréquemment appelé participant. L'appellation de participant peut être appropriée dans le cas d'une personne majeure et apte qui joue un rôle actif dans la réalisation de la recherche. Par contre, convient-elle vraiment à un bébé, à une personne qui a fourni des échantillons ou à une personne devenue inapte? À partir d'une comparaison entre les termes participant et sujet, voyons lequel désigne le plus adéquatement celui qui contribue de sa personne à un projet de recherche. Examinons aussi lequel de ces termes est le plus susceptible d'inspirer au chercheur le respect à l'égard de celui qui contribue de sa personne à son projet de recherche.

Chaque acteur de l'univers de la recherche agit en fonction de sa propre perspective, suivant certaines motivations dont il informe d'autres personnes, mais aussi suivant d'autres motivations qu'il souhaite garder pour lui-même, dans le domaine du non-dit de sa vie personnelle ou professionnelle. Cet état de fait est considéré comme moralement acceptable tant que la relation entre le chercheur et le sujet se conforme aux règles applicables, qu'elles soient légales ou éthiques. En effet, la relation entre le chercheur et le sujet apparaît comme relativement égale ou proportionnée si les mécanismes de protection des sujets sont respectés.

Toutefois, l'égalité formelle relative entre le chercheur et le sujet devient plus difficile à réaliser lorsque le sujet se trouve en situation ou en état de vulnérabilité. L'origine de cette vulnérabilité peut concerner la santé physique ou mentale, la littératie, la relation de couple, l'éducation des enfants, un revers financier, une perte d'emploi, le décès d'une personne chère, l'inadaptation scolaire ou sociale, etc. La condition de vulnérabilité chez une personne peut mettre en péril son intégrité, sa sécurité, son bien-être et l'exercice de ses droits. Nous devons pouvoir compter sur le chercheur et le comité d'éthique de la recherche (CER) pour repérer les personnes en situation de vulnérabilité et leur procurer la protection appropriée.

L'Énoncé de politique des trois Conseils (EPTC2) identifie des personnes qui ont historiquement connu la vulnérabilité : «Les enfants, les personnes âgées, les femmes, les détenus, les personnes souffrant de problèmes de santé mentale et les personnes dont l'aptitude à décider pour elles-mêmes est diminuée» (EPTC2, 2014 : 9). Le dénuement socioéconomique offre aussi un terreau fertile aux abus à l'égard de populations vulnérables. Un exemple classique de ce type d'abus est celui de l'Étude de Tuskegee sur la syphilis².

1. Le philosophe Martin Blais décrit ce besoin que nous avons de nos semblables: «Dire de l'être humain qu'il est social, c'est dire qu'il est fait pour la vie en société en tant qu'il a besoin des autres pour se réaliser, pour s'épanouir ou pour se développer selon toutes ses dimensions». (Blais, 1984, 153).

2. "A classic example of an unjustified offer occurred during the Tuskegee syphilis study. Researchers used various offers to stimulate and sustain the subjects' interest in continued participation ; these offers included free burial assistance and insurance, free transportation to and from the examinations, and a free stop in town on the return trip. Subjects also received free medicines and free hot meals on the days of the examination. The subjects' socioeconomic deprivation made them vulnerable to these overt and unjustified forms of manipulation.» (Beauchamp et Childress, 2013, 139-140). 
Aucune énumération des conditions de vulnérabilité ne peut être exhaustive. Par contre, il est possible d'identifier des conditions inexorables de vulnérabilité, peu importe de qui il s'agit : l'enfance et l'inaptitude. Cette réflexion propose d'ajouter une troisième condition inexorable de vulnérabilité : le désespoir. Notre système juridique vient au secours des enfants et des personnes inaptes en établissant des régimes de protection qui imposent certaines balises aux acteurs chargés de prendre des décisions les concernant. Nos lois prévoient l'intervention de ces régimes de protection dans l'univers de la recherche. Fors les circonstances dans lesquelles leur vie ou leur intégrité est menacée, il n'existe aucune protection juridique pour les personnes acculées au désespoir. Cependant, l'expression de ce constat ne doit pas être interprétée comme un appel pour l'adoption d’une protection légale de toutes les personnes désespérées.

Un événement survenu dans la vie professionnelle du médecin Alexis Carrel montre comment des parents affligés, en désespoir de cause, consentent à une intervention extravagante sur leur bébé. Cette aventure nous conduit à examiner certaines conditions d'existence du consentement libre et éclairé en recherche.

La méprise thérapeutique peut être induite par la manière dont l'équipe de recherche présente son projet, incluant la description du projet contenue dans le formulaire de consentement, ou être le fruit des espoirs du sujet. En fait, le phénomène de la méprise thérapeutique ne provient pas nécessairement du comportement du chercheur. Il est bien souvent le résultat d'une incompréhension de la différence entre la finalité de la recherche et la finalité de la clinique. II arrive aussi que le sujet développe une pensée magique ou exagérément optimiste. Est-il envisageable que le chercheur adopte des attitudes qui réduisent les possibilités de méprise thérapeutique?

Le chercheur soucieux de recruter des sujets qui se représentent adéquatement son projet de recherche et qui y consentent véritablement devrait adopter deux attitudes à leur égard : l'objectivité et la bienveillance. L'ambition de cette réflexion est de montrer que les attitudes d'objectivité et de bienveillance chez le chercheur représentent une solution propre à relever les défis de l'énergie du désespoir et de la méprise thérapeutique.

La teneur de ce propos s'articule autour de quatre événements réels dont les protagonistes essentiels sont des personnes qui se trouvent en situation ou en état de vulnérabilité. Dans la section intitulée «Énergie du désespoir», il est question d'une aventure audacieuse en transfusion sanguine. Dans la section portant sur la méprise thérapeutique, nous rappelons les leçons apprises d'un essai clinique en psychiatrie. Dans la section portant sur l'objectivité, les parents d'un enfant très malade se font offrir la participation à un essai clinique comme représentant la dernière chance pour le jeune patient. Dans la section suivante, une recherche psychosociale auprès de jeunes contrevenants illustre la pertinence de la bienveillance en recherche.

La première partie du développement de ce texte consiste dans un exposé sur la distinction conceptuelle entre la finalité de la recherche et la finalité thérapeutique. La connaissance de cette distinction sera très utile à titre d'entrée en matière et de référence pour la compréhension de la suite du texte, puisque la recherche et la thérapeutique s'y côtoient fréquemment. 


\section{Finalité de la recherche et finalité thérapeutique : distinction conceptuelle}

Du point de vue conceptuel, une intervention à finalité thérapeutique diffère grandement d'une intervention appartenant à la recherche. Le professionnel de la santé qui intervient auprès de son patient dans l'intention de le guérir ou de lui apporter un réconfort agit dans la sphère thérapeutique. Son énergie et sa compétence sont exclusivement tendues vers le bien-être de son patient ou de son client. De son côté, le chercheur qui conçoit ou met en œuvre un projet de recherche a pour premier objectif l'amélioration des connaissances. Toutefois, son attitude tendue vers l'amélioration des connaissances ne signifie pas que le chercheur puisse se montrer indifférent à propos de la condition du sujet de recherche. Au contraire, l'éthique de la recherche contemporaine établit clairement que le respect de l'intégrité et du bien-être du sujet est un impératif incontournable qui a préséance sur les intérêts de la recherche et de la société ${ }^{3}$. C'est pourquoi les bénéfices pour les sujets de recherche doivent être maximisés, et les effets nocifs, réduits au minimum, nous dit l'Organisation des Nations Unies pour l'éducation, la science et la culture (UNESCO)4.

Rappelons toutefois que, dans les faits, certaines initiatives de recherche sont ajoutées aux soins thérapeutiques à titre de voies inédites qui offrent une possibilité de plus longue survie, d'amélioration de la santé, peut-être même de guérison. Des patients acceptent de devenir sujets de recherche parce qu'ils sont porteurs d’un tel espoir. Dans ces cas, la conduite du clinicien embrasse une finalité thérapeutique.

\section{Participant ou sujet de recherche?}

L'EPTC2 pose la question de savoir comment il convient de nommer quelqu'un qui contribue de sa personne au projet de recherche. Participant ou sujet de recherche? Le fait de poser cette question nous rappelle la nécessité de choisir les mots justes ou les plus appropriés dans le contexte de leur emploi. Nous devons les considérer chacun soigneusement dans la visée d'une argumentation limpide qui ne confond pas les termes.

À la question de savoir si une personne doit être appelée participant ou sujet, l'EPTC2 répond qu'il convient de privilégier participant. Voici comment l'EPTC2 définit les participants :

Dans le contexte de la présente politique, les «participants humains» (appelés «participants») sont les personnes dont les données ou les réponses à des interventions, à des stimuli ou à des questions de la part du chercheur ont une incidence sur la question de recherche (EPTC2, 2014 : 14).

L'EPTC2 justifie ainsi sa préférence : participant «reflète mieux l'esprit qui sous-tend les principes directeurs : que les personnes qui choisissent de participer à un projet de recherche y jouent un rôle plus actif que celui qu'évoque le terme "sujet" ». Un autre passage de l'EPTC2 vient préciser, dans le contexte des données et du matériel biologique humain, la notion de participant : «une personne dont les données ou le matériel biologique sont utilisés dans la recherche devient un participant»(EPTC2, 2014 : 195). Ici, la personne concernée est

3. «Researchers should ensure the wishes and interests of the donor, where known, are respected at all times and the welfare of research participants should always take precedence over the interests of science and society.» (MRC, 2014, 5).

4. «Dans l'application et l'avancement des connaissances scientifiques, de la pratique médicale et des technologies qui leur sont associées, les effets bénéfiques directs et indirects pour les patients, les participants à des recherches et les autres individus concernés, devraient être maximisés et tout effet nocif susceptible d'affecter ces individus devrait être réduit au minimum.» (UNESCO, 2005, art. 4). 
un participant même si ce que le chercheur utilise est détaché de sa personne, comme des données ou du matériel biologique.

Comment utilise-t-on le terme participant dans le langage quotidien? Le dictionnaire nous renvoie au verbe participer, pour lequel on trouve les définitions suivantes: «1. Avoir part à quelque chose: Participer aux profits d'une entreprise. - 2. Prendre part à une action, à un sentiment, etc. : Participer aux délibérations. Participer à la joie générale» (Larousse, 1997, 5504). Il s’agit d'avoir part, de prendre part.

Selon la nature de la recherche, l'intensité de la contribution du sujet peut varier considérablement : recevoir un médicament dans le cadre d'un essai clinique, utiliser une nouvelle aide technique en réadaptation, prendre part à une entrevue sur les habitudes de vie ou bien confier un prélèvement sanguin et autoriser l'accès à son dossier médical. Contribuer à un essai clinique engage la personne d'une manière plus active et plus entière que de confier du matériel biologique à une biobanque. Dans le cas de la biobanque, il est fort probable que la personne n'entende jamais parler des utilisations qui seront faites de son matériel biologique. Elle ne tient alors aucun rôle actif. II se peut aussi qu'une biobanque offre à ses sujets de leur transmettre des informations générales sur ses activités. Même dans ce cas, le rôle du sujet demeure essentiellement passif. Quelquefois, des chercheurs souhaitent accéder à des renseignements recueillis par diverses organisations pour d'autres finalités que celles de la recherche. Ces renseignements peuvent concerner la conduite automobile, les accidents du travail, les caractéristiques d'un milieu environnemental, le dossier académique, les victimes d'actes criminels, le recours à l'assistance sociale, les déplacements internationaux, etc. De tels projets sont réalisés dans l'ignorance totale des personnes que ces renseignements concernent. Il est alors difficile de qualifier ces personnes de participantes.

Dans le réseau de la santé et des services sociaux, il arrive fréquemment que la mise en œuvre d'un projet ne nécessite aucun contact avec des personnes, mais suppose la consultation du dossier de l'usager. L'équipe de recherche y a accès avec l'autorisation du directeur des services professionnels de l'établissement ${ }^{5}$. L'usager dont on aura consulté le dossier ne le saura jamais. Ainsi, appeler cette personne un participant semble une exagération de son rôle factuel. Par ailleurs, l'EPTC2 reconnaît clairement cette distinction entre une personne qui participe directement à une recherche et celle qui y participe parce qu'un chercheur utilise ses données ou son matériel biologique (EPTC2, 2014, 6). Rappelons néanmoins que toutes les personnes qui contribuent à la recherche, quelle que soit l'intensité de leur contribution, méritent qu'on respecte leurs droits, comme le droit à la vie privéé.

Voyons maintenant ce qu'est un sujet. La signification d'un mot peut varier suivant le contexte de son emploi, on dit alors que ce mot est équivoque. Toutefois, il est rare que deux définitions d'un mot décrivent des réalités incompatibles, voire contraires. C'est pourtant le cas du mot sujet, nous dit un dictionnaire philosophique :

Selon l'étymologie latine, le sujet réunit deux significations contraires. D'une part est sujet celui qui est assujetti à un pouvoir, pouvoir du père, du roi, du président, de la loi, de l'ordre, des supérieurs hiérarchiques, etc. D'autre part, le sujet est l'être autonome et conscient, souverain dans la mesure où il peut affirmer sa liberté et endosser la responsabilité de ses actes, quoi qu'il sache, ou ignore, des déterminations naturelles, psychologiques, socio-historiques, politiques, qui constituent sa situation singulière, mais ne le conditionnent pourtant pas de manière définitive (Larousse, 2003 : 995).

5. «Le directeur des services professionnels d'un établissement ou, à défaut d'un tel directeur, le directeur général peut autoriser un professionnel à prendre connaissance du dossier d'un usager, à des fins d'étude, d'enseignement ou de recherche.» (LSSS, art 19.1)

6. «Toute personne a droit au respect de sa vie privée.» (Charte, art 5) 
Un dictionnaire historique de la langue française relève les deux mêmes significations. Considérons d'abord la signification de l'être assujetti. Le mot sujet constitue un «emprunt au latin subjectus «soumis», "assujetti», «exposé» et «voisin, proche», participe passé passif de subjicere «placer dessous», «amener à proximité de», «soumettre, subordonner»» (Le Robert, 1998, 3686). Maintenant, soulignons le sens de l'être autonome et conscient: «Le mot entre dans le vocabulaire philosophique au début du XIXe s. d'après l'allemand Subjekt (Kant), de même origine, désignant l'être pensant, considéré comme le siège de la connaissance, par opposition à un objet» (Le Robert, 1998, 3686). Envisagé au sens de l'être soumis, sujet prend l'une des significations du mot objet: «Chose inerte, sans pensée, sans volonté et sans droits, par opposition à l'être humain : On nous traite comme des objets» (Larousse, 1997, 5236). L'usage des mots dans le langage quotidien finit par produire une assimilation entre sujet au sens de l'être soumis et objet à titre de chose inerte. Personne ne soutiendrait qu'en recherche sur la santé humaine, il est admissible de considérer la personne comme si elle était un objet. Il est compréhensible que l'on préfère participant à sujet pour désigner la personne qui accepte de contribuer à la recherche si la signification donnée au mot sujet évoque la soumission ou l'idée d'une personne réduite au statut d'objet.

Les dictionnaires contiennent des définitions de sujet qui renvoient à la recherche scientifique. Un dictionnaire historique montre les différents emplois du terme sujet : un être vivant dont les caractéristiques permettent qu'on réalise sur lui des expériences, un être vivant soumis à l'observation, un cadavre utilisé en anatomie, un être vivant que l'on soigne ${ }^{7}$. Dans ce sens du terme sujet, une personne peut être un sujet d'étude ou d'expérimentation. Un dictionnaire encyclopédique propose de définir sujet comme un «être vivant soumis à l'observation» (Larousse, 1997, 6993). La personne humaine devient sujet de recherche lorsque son être physique, psychique ou social est étudié dans le contexte d'une initiative ayant pour finalité le progrès des connaissances. Suivant cette dernière définition de dictionnaire, le terme sujet s’applique à tous les cas de figure, indépendamment du caractère plus ou moins actif de la contribution de la personne concernée.

L'EPTC2 comporte deux versions officielles : l'une est française, et l'autre, anglaise. Les dernières remarques à propos de l'appellation de participant ou de sujet sont fondées sur les définitions de la langue française. Qu'en est-il de l'anglais? Voyons l'équivalent anglais de chaque mot-clé français employé : participant - participant; sujet - subject; objet - object. Ces dyades ne forment pas de faux amis ${ }^{8}$, puisque les diverses significations de ces termes sont similaires d'une langue à l'autre, comme nous le constatons ci-dessous.

Un participant prend part à quelque chose. En anglais, le participant est «A person who takes part in something» (Oxford, en ligne).

Le terme subject prend les trois significations suivantes, parmi d'autres:

- «Under the authority of» (adjectif);

- «A thinking or feeling entity; the conscious mind; the ego, especially as opposed to anything external to the mind» (nom);

- A person who is the focus of scientific or medical attention or experiment» (nom) (Oxford, en ligne).

\footnotetext{
7. «Parallèlement, sujet désigne un être vivant dont les caractéristiques conviennent pour réaliser certaines expériences (mil. XVIe s.), plus généralement un être vivant soumis à l'observation (v. 1560), spécialement un cadavre utilisé pour l'étude de l'anatomie, et un être vivant que l'on soigne (1778).» (Le Robert, 1998, 3687-3688).

8. Les faux amis anglais-français sont des mots de la langue anglaise qui, par leur forme, en rappellent d'autres dans la langue française, mais dont les significations diffèrent. Par exemple, librairie et library sont de faux amis.
} 
La notion d'objet manifeste, en français, la chose inerte. Selon le contexte, le mot anglais object prend ce sens: «A thing external to the thinking mind or subject». (Oxford, en ligne)

La discussion afin de déterminer s'il faut employer participant ou sujet se pose dans les mêmes termes en anglais et en français. Les éléments conceptuels qui organisent la pensée sont virtuellement identiques.

Appliquant au terme sujet les définitions selon lesquelles il est un être pensant, autonome et conscient, l'utilisation de ce terme ne mutile en rien la dignité de la personne humaine. Le statut de sujet introduit une insistance concernant le devoir de respecter la capacité de décider de la personne, exactement dans l'esprit de l'EPTC2:

Respecter les personnes, c'est reconnaître la valeur intrinsèque de tous les êtres humains; c'est aussi reconnaître que chacun a ainsi droit au respect et à tous les égards qui lui sont dus [...]. L'autonomie comprend la capacité de délibérer au sujet d'une décision et d'agir en conséquence. Respecter l'autonomie, c'est reconnaître la capacité de jugement d'une personne et faire en sorte que la personne soit libre de choisir sans ingérence (EPTC2, 2014, 6-7).

Employé au sens de l'être autonome et conscient, le terme sujet affirme immédiatement la dignité de toute personne et rappelle au milieu de la recherche que chacun mérite d'être respecté en considération de cette dignité inhérente.

\section{Énergie du désespoir}

Un dictionnaire encyclopédique propose cette définition du désespoir : «Perte de toute espérance, abattement total de quelqu'un qui a cessé d'espérer; affliction profonde, détresse, désespérance : Sombrer dans le désespoir» (Larousse, 1997, 2188). Si nous donnons créance à ce dictionnaire, il ne faut pas imaginer que le chagrin du désespoir engendre uniquement un état de torpeur ou d'inaction. L'expression en désespoir de cause signifie «faute de pouvoir recourir à un autre moyen» (Larousse, 1997, 2189). Énergie du désespoir dépeint la «résolution violente qu'inspire une situation désespérée» (Larousse, 1997, 2189). Le désespoir peut faire tendre quelqu'un vers une action énergique et obstinée, même si le moyen retenu n'est pas idéal. C'est ce que montre le récit qui suit.

Alexis Carrel, chirurgien et biologiste français, a vécu de 1873 à 1944. Il fut un pionnier de la chirurgie vasculaire. Il a imaginé de nouvelles techniques chirurgicales et réalisé la mise au point d'aiguilles très délicates pour améliorer la qualité des sutures. Afin de développer son habileté manuelle, il devint apprenti auprès de celle qui avait la réputation d'être la meilleure brodeuse artistique à Lyon, madame Leroudier. Le Nobel de médecine fut décerné au Dr Carrel en 1912. Ses travaux l'ont conduit à Montréal, Chicago, puis au Rockefeller Institute for Medical Research à New York.

En 1908, alors qu'Alexis Carrel se trouve à New York, le Dr Adrian Lambert et ses deux frères chirurgiens lui demandent de sauver un bébé. La femme d’Adrian Lambert vient de mettre au monde une petite fille qui perd du sang par le nez et la bouche depuis plusieurs jours. Le Dr Carrel répond qu'il n'est pas autorisé à pratiquer la chirurgie dans l'État de New York: «l have no license to practice surgery in the state of New York. My patients are only my dogs and cats» (Douglas, 2002, 31). Le Dr Lambert s'engage à prendre l'entière responsabilité légale à ce propos. Il insiste pour que le Dr Carrel le suive immédiatement: «Lambert promised that he would take full legal responsibility, if only Carrel would please come-at once» (Douglas, 2002, 31). Le Dr Carrel pratique une transfusion sanguine directe d'une artère du poignet gauche du père dans la veine poplitée du bébé, derrière le genou, par une suture artère-veine. La petite fille prend aussitôt du mieux. 
Le caractère tout à fait exceptionnel de cet événement saute aux yeux. Nous sommes en 1908 et nous pouvons supposer que les protagonistes se sont comportés au meilleur de leurs capacités à ce moment de leur vie. L'intervention envisagée ne fait pas l'objet d'un projet de recherche qui aurait été soigneusement réfléchi et qui interviendrait comme aboutissement à des travaux antérieurs; nous assistons plutôt à une entreprise humanitaire désespérée.

Pour quelles raisons le Dr Adrian Lambert insiste-t-il tellement afin qu'une intervention aussi hasardeuse soit mise en œuvre? Le bébé est sa fille, et il tient à tout faire pour lui sauver la vie, puisque l'alternative semble être d'attendre son décès. Nous pouvons aussi penser que la perte de cette enfant serait désastreuse pour sa mère. L'état des connaissances ne permet pas d'envisager les séquelles possibles de cette intervention. En outre, la condition future du bébé, sa qualité de vie, ne peut pas entrer dans les considérations entre les bénéfices et les inconvénients.

Du point de vue du Dr Carrel, une intervention, même au bénéfice très incertain, offre une réponse immédiate au désespoir des parents. Si le bébé survit, l'intervention aura épargné aux parents le drame de perdre leur enfant, du moins à court terme. De plus, la situation offre au Dr Carrel la possibilité d'oser une intervention sans précédent, et qui lui permet de satisfaire sa curiosité scientifique. Enfin, cette transfusion sanguine innovatrice pourrait contribuer au progrès des connaissances.

Il ne saurait être question d'évaluer la conduite des quatre médecins concernés selon les normes déontologiques actuelles. Cependant, considérer cet événement sous l'angle de la déontologie applicable maintenant met en relief les normes qui nous semblent les plus fondamentales. Bien que les faits se soient déroulés à New York, nous utiliserons la Loi médicale et le Code de déontologie des médecins du Québec, car nous relevons de la juridiction québécoise. Tenons-nous-en uniquement à quelques-unes de leurs dispositions, puisque la considération des autres règles applicables serait interminable.

Premièrement, son statut professionnel ne permettait pas au Dr Carrel d'exercer la médecine à New York. À cette époque, comme aujourd'hui, le Dr Carrel risquait de se faire reprocher l'exercice illégal de la médecine. Dans le contexte québécois actuel, l'exercice illégal de la médecine fait l'objet de l'article 43 de la Loi médicale. L'engagement du Dr Lambert d'en prendre l'entière responsabilité légale ne se substitue pas au permis d'exercer la médecine. La demande empressée du patient ou celle de son représentant légal dans le but d'obtenir une intervention n'autorise personne à exercer la médecine.

Deuxièmement, le médecin doit «engager pleinement sa responsabilité civile» dans sa pratique professionnelle. L'engagement du père du bébé à prendre la responsabilité légale pour la suite des événements ne permet pas au médecin d'éluder sa responsabilité pour les actes professionnels qu'il posera?.

Troisièmement, l'article 28 du Code de déontologie des médecins requiert que le médecin obtienne un consentement libre et éclairé avant de dispenser un soin :

Le médecin doit, sauf urgence, avant d'entreprendre un examen, une investigation, un traitement ou une recherche, obtenir du patient ou de son représentant légal, un consentement libre et éclairé.

9. «Le médecin doit, dans l'exercice de sa profession, engager pleinement sa responsabilité civile. Il ne peut l'éluder ou tenter de l'éluder, ni requérir d'un patient ou d'une personne une renonciation à ses recours en cas de faute professionnelle de sa part.» (Code de déontologie des médecins, art 11) 
La transfusion sanguine effectuée sur le bébé se présente comme une terra incognita pour les quatre médecins présents. Au-delà de l'hémorragie dont souffrait l'enfant, il devait leur être très difficile de s'avancer pour constater un diagnostic ou envisager un pronostic. La conduite d’Adrian Lambert nous invite à penser que le consentement des parents a été libre, néanmoins faiblement éclairé en raison de la réalité des connaissances médicales à cette époque. Le contexte humain dans lequel se trouvait le couple désespéré d'Adrian Lambert consistait dans un choix entre le décès certain de leur petite fille ou la tentative de la sauver au moyen d'une intervention cliniquement hasardeuse. Ainsi formulée, la question impose de choisir entre la vie et la mort, et elle s'adresse à quelqu'un qui considère n'avoir rien à perdre en osant une intervention. Le problème posé serait passablement différent s'il était possible de tenir compte de la qualité de vie future de l'enfant dans l'éventualité de sa survie.

Si nous considérons cette transfusion sanguine d'après les règles applicables en matière de recherche, une disposition de la déontologie médicale attire forcément notre attention : celle qui requiert l'approbation préalable d'un comité d'éthique de la recherche qui requiert l'approbation préalable d'un CER, étant entendu que celui-ci fonctionne dans le respect des normes en vigueur ${ }^{10}$. Cette exigence du respect des normes en vigueur opère un renvoi à des documents normatifs internationaux, canadiens et québécois ${ }^{11}$. Partant, les comportements exigés des médecins en matière de recherche ne se limitent pas aux obligations que contient le Code de déontologie des médecins. Il va de soi que l'intervention du Dr Carrel n'ait pas fait l'objet d'un examen par un CER. Cette intervention n'a donc pas été évaluée par une instance indépendante en fonction de sa validité scientifique, des bénéfices espérés et des risques anticipés. De nos jours, un médecin dont la conduite s'apparenterait à celle du Dr Carrel durant ces événements serait perçu comme ayant démontré une audace et une insouciance inacceptables.

L'histoire du Dr Carrel, du couple Lambert et de la petite fille met en relief certaines questions que pose l'obtention d'un consentement libre et éclairé lorsque l'interlocuteur du chercheur se trouve dans un état de désespoir. Le patient est-il en mesure de retenir et de comprendre correctement les informations reçues? Perçoit-il la réalité de ce que lui propose le chercheur ou se trouve-t-il dans un état psychique qui exagère le bénéfice possible de la recherche? L'équipe de recherche présente-t-elle le projet comme elle le devrait? Ces questions nous conduisent à discuter de la méprise thérapeutique et de la possibilité que cette méprise invalide le consentement. Quel type de conduite le chercheur peut-il adopter pour réduire l'incidence et l'intensité de la méprise thérapeutique?

\section{Méprise thérapeutique}

Le professeur américain de psychiatrie Paul S. Appelbaum a publié de nombreux articles sur la notion de méprise thérapeutique (therapeutic misconception). Une de ces publications, datant de 1987, décrit comment un patient perçoit sa contribution à un projet de recherche. II s'agit d'un jeune homme qui vient de tenter de se suicider, qui a une longue histoire de relations tumultueuses et qui éprouve des difficultés à maîtriser ses impulsions (impulses). Un psychiatre lui offre de participer à un projet de recherche ayant pour objectif de

10. «Le médecin doit, avant d'entreprendre sa recherche sur des êtres humains, obtenir l'approbation du projet par un comité d'éthique de la recherche qui respecte les normes en vigueur, notamment dans sa composition et dans ses modalités de fonctionnement. Il doit également s'assurer que tous ceux qui collaborent avec lui à la recherche soient informés de ses obligations déontologiques.» (Code de déontologie des médecins, art 31).

11. «L'éthique de la recherche en santé humaine est institutionnalisée, elle comporte des instances présentes notamment dans les universités et dans les établissements de santé et de services sociaux. Au cœur de ces instances, nous trouvons les comités d'éthique de la recherche (CÉR) et les comités scientifiques.» (Giroux, 2010, 114) 
constater si des médicaments peuvent aider au traitement de sa condition. Le patient accepte d'y participer : «Yes, I'm willing to do anything that might help me» (Appelbaum et coll., 1987, 20). Quelques jours plus tard, le psychiatre rencontre à nouveau le jeune homme afin de lui donner plus d'informations sur le projet de recherche. Le psychiatre expose que deux médicaments et un placebo seront utilisés. Les médicaments et le placebo seront attribués au hasard, et même le chercheur ne saura pas ce que chacun reçoit. Le patient écoute les informations et signe le formulaire. Selon toute apparence, le consentement exprimé est libre et éclairé. Cependant, quand on demande au patient pourquoi il a consenti à la recherche, il répond que la médication qu'il recevra sera celle qui peut le mieux améliorer sa condition. Il se figure que le projet de recherche est conçu pour lui venir en aide directement.

Cette description des pensées du jeune homme montre que le phénomène de la méprise thérapeutique ne pourrait pas survenir dans le cadre d'un projet de recherche qui utilise des sujets en bonne santé. La méprise thérapeutique est uniquement possible lorsque la recherche recrute des personnes atteintes d'une pathologie.

La représentation que le jeune homme se fait du projet de recherche ne correspond pas à la réalité. La croyance erronée de la personne affectée par la méprise thérapeutique veut que le projet de recherche ait été conçu pour son bien-être à elle et nie la possibilité que sa contribution à la recherche puisse comporter des inconvénients majeurs ${ }^{12}$. La méprise thérapeutique désigne un état d'esprit présent chez la personne qui accepte de contribuer à un projet de recherche. II s'agit d'une incompréhension de sa contribution à la recherche, puisque la personne voit son bien particulier comme un facteur central dans l'intention du chercheur et qu'elle se trouve en déni face aux inconvénients possibles.

Il importe de rappeler que, dans ce cas, la méprise du patient ne provient pas d'indications floues ou mensongères de la part du psychiatre. C'est le patient lui-même qui teinte sa compréhension des tournures de son espérance. Or, il n'existe aucun moyen qui permette à qui que ce soit d'exercer une emprise sur les représentations qu'entretient cette personne. Toutefois, l'équipe de recherche peut vérifier la compréhension du sujet et fournir des explications de manière à contourner la nervosité ou la faible littératie de cette personne.

Le philosophe Alain nous parle du recrutement militaire à une certaine époque. Beaucoup de jeunes hommes pauvres, mais énergiques semblaient se vendre pour une solde et un uniforme, alors qu'ils s'engageaient d'abord pour des motifs de gloire:

Les recruteurs d'autrefois étaient des marchands d'esclaves. Voltaire nous conte dans Candide l'aventure d'un de ces grands imbéciles qui se vendaient pour un bon déjeuner et une petite masse de monnaie, avec la promesse d'une solde et d'un uniforme. En réalité, ces hommes vigoureux, à l'âge où on s'ennuie, avaient envie de gloire et de voir du pays; sans compter que souvent ils étaient bien pauvres et mangeaient trop pour des pauvres. Je crois pourtant que les motifs de gloire étaient les plus forts, comme ils sont aujourd'hui (Alain, 1970, 1057).

Le jeune homme pauvre ne s'engage pas chez les militaires dans le but de se transformer en chair à canon. Il rêve du mieux : voir du pays et rentrer chez lui glorieux. La même espérance pour le mieux semble entraîner le

12. «Yet when the patient is asked why he agreed to be in the study, he offers some disquieting information. The medication that he will receive, he believes, will be the one most likely to help him. He ruled out the possibility that he might receive a placebo, because that would not be likely to do him much good. In short, this man, now both a patient and a subject, has interpreted, even distorted, the information he received to maintain the view - obviously based on his wishes - that every aspect of the research project to which he had consented was designed to benefit him directly. This belief, which is far from uncommon, we call the 'therapeutic misconception.' To maintain a therapeutic misconception is to deny the possibility that there may be major disadvantages to participating in clinical research that stem from the nature of the research process itself.» (Appelbaum et coll., 1987, 20). 
sujet à croire que la recherche lui offrira, à lui, les meilleurs soins, en dépit des explications très différentes qui lui sont communiquées. Le phénomène de la méprise thérapeutique se produit lorsque la personne concernée ne fait pas la distinction entre la finalité thérapeutique et la finalité de la recherche. Le résultat de cette confusion est de percevoir la contribution à une recherche comme étant de nature thérapeutique.

Une interprétation rigoureuse du critère de la compréhension appropriée a pour effet d'invalider le consentement exprimé, puisque, dans son for intérieur, la personne ne consent pas véritablement à une recherche ${ }^{13}$. Comme l'équipe de recherche n'est pas toujours en mesure de constater cette incompréhension du sujet, nous pouvons supposer que des projets de recherche sont réalisés avec un certain nombre de personnes qui n'ont pas compris ce dont il s'agit. Par ailleurs, quelle devrait être la conduite de l'équipe de recherche qui constate l'absence du caractère éclairé d'un consentement? Serait-il paternaliste d'annoncer au sujet qu'il ne participera pas à la recherche en raison de l'absence du caractère éclairé de son consentement?

L'EPTC2 propose une approche susceptible de limiter le nombre de circonstances dans lesquelles survient la méprise thérapeutique. Il s'agit par exemple de faire en sorte que l'équipe soignante œuvrant auprès de la personne n'intervienne pas ou intervienne aussi peu que possible dans la conduite de la recherche. Lorsque le clinicien traitant participe à la recherche, les responsables de ce projet doivent indiquer quelles mesures seront mises en œuvre pour réduire la possibilité de méprise thérapeutique ${ }^{14}$.

\section{Objectivité et bienveillance chez le chercheur}

Deux attitudes du chercheur et de son équipe contribuent à la possibilité, pour le sujet potentiel, de comprendre adéquatement la recherche et d'y participer en exprimant une volonté libre et éclairée : l'objectivité et la bienveillance. Au sens de son emploi dans ce texte, une attitude consiste dans une «manière d'être qui manifeste certains sentiments» (Larousse, 1997, 539). L'attitude de l'objectivité manifeste l'objectivité, l'attitude de la bienveillance manifeste la bienveillance.

\section{Objectivité}

Voyons d'abord l'objectivité. D'entrée de jeu, précisons que l'objectivité dont il est ici question ne constitue pas une qualité de la connaissance, mais une attitude du chercheur à l'égard de celui qui contribue de sa personne à la recherche, le sujet.

La clarté de ce propos requiert une définition précisant la signification du mot objectivité dans le présent contexte. La notion d'objectivité prend diverses significations, relativement similaires. Une première signification se rapporte à l'indispensable collaboration entre les êtres humains. La simple satisfaction de nos besoins

13. "The 'therapeutic misconception' is a widely discussed problem of informed consent that must be addressed in research, where subjects may fail to distinguish between clinical care and research and may fail to understand the purpose and aim of research, thereby misconceiving their participation as therapeutic in nature. In a stringent interpretation of the standard of adequate understanding, the therapeutic misconception invalidates a subject's consent because he or she is not truly consenting to participation in research.» (Beauchamps et Childress, 2013, 133).

14. «En règle générale, on peut réduire la possibilité que les participants ne se méprennent sur l'aspect thérapeutique d'un essai et éviter ainsi de créer un malentendu ou de fausses attentes, si les cliniciens qui dispensent des soins de santé au patient interviennent aussi peu que possible dans le recrutement et le processus de consentement. Idéalement, les fonctions de traitement et de recherche seront assumées par des personnes différentes, mais dans certains cas, il sera dans l'intérêt des participants d'impliquer leur clinicien traitant au processus de recrutement et de consentement. Dans ces cas, le dossier du projet de recherche proposé doit indiquer les mesures supplémentaires qui seront prises pour réduire la possibilité de méprise thérapeutique.» (EPTC2, 2014, 183). 
vitaux nous impose de nous adapter à la réalité, de communiquer avec d'autres personnes et de coopérer avec elles sur le plan pratique, nous dit le philosophe José Antonio Marina. La mise en œuvre de toutes ces composantes requiert la configuration, dans la conscience du sujet, d'un espace objectif, commun, interpersonnel et durable. Le dialogue, par exemple, est possible seulement si on peut sortir, au moins de façon partielle, de son monde privé pour accéder à l'objectivité, un espace interpersonnel que personne ne possède, et accessible à tous ${ }^{15}$. Prise dans ce sens, l'objectivité résulte de la sortie de soi-même. II s'agit d'adopter une posture psychique qui renonce au confort de la subjectivité et qui s'efforce d'entrer en contact avec l'autre au moyen d'un langage commun.

Un dictionnaire terminologique propose une définition de l'objectivité qui la considère comme une préoccupation : «Souci constant de faire abstraction des mobiles et des opinions personnelles dans le jugement» (OLF, en ligne). Cette définition met en évidence l'intention de la personne pour laquelle il importe de faire abstraction de ses opinions personnelles dans le jugement qu'elle pose. Il s'agit d'un «souci», d'une préoccupation, plutôt que d'un accomplissement. L'objectivité ainsi définie est l'affaire de toutes les activités professionnelles : de la pratique médicale à la sociologie, de la pratique du droit à l'ingénierie. Définie comme une préoccupation, l'objectivité ne présente point le caractère de la certitude mathématique ni son auréole d'inaccessibilité pour les chercheurs des autres sciences. À titre de préoccupation, l'objectivité est universellement réalisable, au prix d'une attention soutenue.

Un autre dictionnaire formule une définition qui exprime un niveau d'exigence plus élevé que celui de la préoccupation, en ce que la personne concernée porte effectivement un jugement sans qu'interviennent ses opinions ou ses préférences personnelles : «Qualité de quelqu'un, d’un esprit, d'un groupe qui porte un jugement sans faire intervenir des préférences personnelles: Diriger avec objectivité des débats.» (Larousse, 1997, 5236). Nous trouvons des illustrations de cette objectivité dans la décision juste d'un tribunal et dans l'impartialité du président d'une assemblée.

Enfin, l'objectivité peut nommer une caractéristique désirable de l'activité intellectuelle. Il s'agit alors de la «Qualité de ce qui est conforme à la réalité, d'un jugement qui décrit les faits avec exactitude : L'objectivité d'un récit» (Larousse, 1997, 5236). Parvenir à une description exacte du réel constitue un défi considérable pour tous les domaines de connaissance. De l'astrophysique à l'anthropologie, les chercheurs observent et tentent d'expliquer divers phénomènes. Les abonnés à la littérature scientifique constatent rapidement que telle description d’un phénomène céleste peut être améliorée, que tel médicament provoque des réactions positives imprévues ou que telle intervention de la psychologie se révélerait peut-être plus efficace si elle était modifiée en fonction d'une approche innovatrice. La conquête de nouveaux savoirs se révèle difficile dans un contexte où les chercheurs conduisent leurs travaux en tâchant de décrire les faits avec exactitude. Néanmoins, l'évolution du savoir serait un projet absurde si chacun y allait de sa subjectivité particulière.

Maintenant, illustrons l'objectivité comme attitude du chercheur à l'égard du sujet.

Afin de complexifier un petit peu notre enjeu, situons-le en pédiatrie, à propos d'un enfant de deux ou trois ans. La décision de participer ou non à une recherche appartient aux parents, bien que le sujet éventuel ne soit aucun d'eux, mais leur enfant. Les parents d'un enfant atteint d'une maladie grave à qui on offre de participer à un essai clinique peuvent être vivement tentés d'y consentir si cet essai clinique leur est présenté comme l'inter-

15. «Las necesidades vitales imponen una adecuación a la realidad, una comunicación con otros seres y una cooperación con ellos en el plano práctico. Todas estas cosas exigen la configuración en la conciencia del sujeto de un espacio objetivo, común, interpersonal y firme. El diálogo, por ejemplo, sólo es posible cuando puede salirse, aunque sea fragmentariamente, del mundo privado para acceder a la objetividad, una tierra de nadie utilizable por todos.» (Marina, 2004, 52). 
vention de la dernière chance. Ajoutons à cette justification le postulat selon lequel des parents qui veulent le mieux pour leur enfant devraient évidemment y consentir. Les subtilités des explications données aux parents exercent une influence déterminante sur leur décision, certes, de même que sur la qualité de leur décision. Dans les pires cas, le chercheur qui présente son projet de recherche de manière à obtenir un consentement subordonnera, tôt ou tard, son objectivité à son intérêt. Face à des parents très inquiets, voire désespérés, l'objectivité du chercheur qui leur transmet les renseignements leur permet de consentir à une intervention ou de la refuser en sachant de quoi il retourne.

Comme de raison, les parents auront tendance à rechercher l'opinion du médecin traitant, car celui-ci connaît leur enfant et détient des connaissances scientifiques de même qu'une expérience clinique. De plus, même si son activité le rapproche des parents, ce médecin n’est pas engagé personnellement dans la situation familiale. Il lui est plus facile qu'à un proche d'exprimer un avis à la fois détaché et conforme à ce que la réalité scientifique rend possible. Le rôle du médecin traitant est d'utiliser sa connaissance au service de son petit patient et des parents : «Dans une situation aussi difficile, le médecin spécialiste jouit d'un grand avantage sur les parents : lui, il sait. Certes, il ne sait pas tout, mais il détient des connaissances» (Dorval et Hébért, 2005, 59) sur les jeunes patients en général et sur les particularités de l'enfant dont il s'agit. Les parents lui font spontanément confiance.

Le souci constant de faire abstraction de ses opinions et de ses mobiles personnels requiert du chercheur qu'il s'efforce de ne pas se laisser influencer par son a priori intellectuel ou son intérêt. Par exemple, il peut être tentant pour un chercheur dans le champ biomédical d'appliquer une interprétation créative des critères d'inclusion à un projet de recherche. C'est la raison pour laquelle l'Association médicale canadienne estime que la rémunération en conséquence du recrutement n'est pas éthique. Il en va de même pour l'établissement de quotas en rapport avec la rémunération ${ }^{16}$. Dans le même esprit, l'EPTC2 demande au CER d'examiner les modalités de paiement afin d'éviter un recrutement inapproprié ${ }^{17}$. Il reste que les instruments les plus efficaces pour prévenir de tels abus demeurent l'objectivité et l'honnêteté du chercheur qui s'emploie à mettre de côté son intérêt pécuniaire.

Maintenant, supposons qu'un chercheur d'une discipline des sciences humaines ait développé la conviction que, dans leur propre intérêt, les itinérants devraient faire l'objet d'interventions psychosociales beaucoup plus autoritaires et contraignantes. Pour convaincre son milieu professionnel de la justesse de sa conviction, le chercheur élabore un projet de recherche dont les instruments de collecte de données et les outils d'analyse comportent des biais destinés à confirmer son approche de l'itinérance. Dans sa description du projet aux sujets potentiels, ce chercheur procédera sans objectivité et, de ce fait, il obtiendra des consentements non éclairés.

La nécessité morale de l'objectivité au sens d'une préoccupation face aux préférences et aux opinions personnelles permet au chercheur de repousser la tentation de l'avidité et l'appel de la vanité. Une carrière scienti-

16. «Are there financial incentives for researchers to recruit participants? Finders' fees and referral fees should not be accepted; they are unethical. Are there rewards for meeting quotas? This may unduly influence recruitment.» (CMA, 2008, 17).

17. «Le CER examinera les budgets pour vérifier qu'aucun paiement inapproprié ou que d'autres dépenses inexpliquées ne permettent de soupçonner l'existence de conflits d'intérêts. En outre, il étudiera les dispositions de paiement pour veiller à ce qu'elles n'entraînent aucun incitatif de recrutement rapide, inacceptable sur le plan éthique, au détriment d'une étude minutieuse des caractéristiques recherchées chez les participants éventuels. Dans le cas de paiements déraisonnables ou d'incitations indues, le chercheur, et parfois son établissement d'attache, pourrait se trouver dans une situation de conflit entre la recherche d'une rémunération financière maximale, d'une part, et la protection des participants ainsi que le respect des objectifs et exigences scientifiques du projet de recherche, d'autre part.» (EPTC2, 2014, 105). 
fique peut être aussi politique, compétitive et féroce que toute autre carrière. Elle est parsemée d'invitations à utiliser des expédients faciles ${ }^{18}$. Nous rejoignons ici notre point de départ en compagnie du philosophe Marina: L'accès à l'objectivité requiert l'adoption de la posture psychique de celui qui sort de lui-même pour accéder au monde extérieur, à des relations interpersonnelles et à des pensées autres qu'égocentriques.

\section{Bienveillance}

Passons maintenant à la bienveillance. Quiconque s'intéresse à la bioéthique rencontre naturellement quatre principes largement connus : respect de l'autonomie, bienfaisance, non-malfaisance et justice. Il est régulièrement question du principe de bienfaisance au cours de lectures ou lors de conversations à propos de la relation thérapeutique. Quant à elle, la bienfaisance doit être distinguée de la bienveillance, car il existe une différence appréciable entre ces deux termes, que l'on risque de confondre par mégarde. L'étymologie nous renseigne adéquatement sur la distinction à établir entre eux. La bienfaisance (bien et faire) consiste dans l'action de faire du bien, alors que la bienveillance (bien et vouloir) (Larousse, 2003, 392-393) est l'attitude de la personne qui montre «de bonnes dispositions à l'égard de quelqu'un» (Larousse, 1997, 826). Comme il a déjà été expliqué, à la différence du contexte thérapeutique dont la visée est de procurer un bien au patient, le contexte de la recherche a pour objectif l'amélioration des connaissances. Il se peut que sa contribution à une recherche améliore la condition d'une personne, mais ce résultat n'est pas le premier objectif de la démarche du chercheur. Conséquemment, on ne peut exiger du chercheur qu'il contribue au bien-être des sujets, qu'il soit bienfaisant, dans la réalisation de son projet:

Il arrive souvent qu'un projet de recherche n'offre guère ou n'offre pas de bénéfices directs aux sujets. En fait, les principaux bénéfices qui découlent de la plupart des projets de recherche concernent la société et l'avancement des connaissances (EPTC2, 2014, 22)

Jusqu'où la bienveillance du chercheur est-elle susceptible de se manifester? Se trouve-t-elle adéquatement inspirée lorsqu'elle tient compte de la perspective ou de la personnalité du sujet? La personnalisation d'une activité consiste dans l'«adaptation d'un produit, d'un service, d'un logement, etc., à la personnalité de celui à qui il est destiné» (Larousse, 1997, 5623). Est-il vraisemblable que, dans l'élaboration et la réalisation d'un projet de recherche, le chercheur tienne compte de la personnalité du sujet, de son point de vue? Le défi de la bienveillance en recherche apparaît au premier chapitre de l'EPTC2, lorsque ce document normatif affirme que les chercheurs et les CER ont l'obligation de tenir compte de la perspective du participant:

Dans l'élaboration et la réalisation d'une recherche ou lors de son évaluation éthique, les chercheurs et les CER ont l'obligation de tenir compte de la perspective du participant. En conséquence, il pourrait être nécessaire de se pencher sur divers contextes (par exemple social, économique, culturel) qui façonnent la vie du participant pour évaluer convenablement les implications de la recherche au regard des principes directeurs (EPTC2, 2014, 11).

Ce défi de la bienveillance apparaît une seconde fois dans l'EPTC2, concernant les avantages, les risques ou les inconvénients que comporte un projet. L'EPTC2 recommande clairement aux chercheurs et aux CER d'adopter l'attitude appelée ici bienveillance:

18. «Science, I had come to learn, is as political, competitive, and fierce a career as you can find, full of the temptation to find easy paths.» (Kalanithi, 2016, 100). 
Les participants peuvent percevoir les préjudices d'une façon différente des chercheurs. Ils peuvent aussi réagir de façons différentes à la recherche. Les chercheurs et les CER devraient autant que possible tenter d'évaluer les préjudices du point de vue des participants (EPTC2, 2014, 22).

Le premier devoir de la bienveillance est certainement de protéger les sujets contre tout risque inutile ou évitable. Comme pour l'accès à l'objectivité, l'exercice de la bienveillance requiert l'adoption de la posture psychique de celui qui sort de lui-même. Ici, le chercheur sort de lui-même en s'enquérant du point de vue d'une autre personne, le sujet.

Il peut être aisé de saisir le point de vue du sujet, mais plus complexe d'en tenir compte avec justesse, même dans une intention bienveillante. Une illustration qui comporte sa part d'humour nous le montre bien. Un psychologue souhaite réaliser un projet de recherche auprès de jeunes contrevenants qui se trouvent en centre jeunesse. Sa recherche comporte un entretien avec chaque sujet. Pour inciter les jeunes à participer et en guise de reconnaissance pour leur contribution, le chercheur leur offre un sac de chips, une boisson gazeuse et une barre de chocolat. Il faut savoir que l'offre alimentaire du centre jeunesse ne comporte ni croustilles, ni boissons gazeuses, ni barres de chocolat. Il est manifeste que ces régals exerceront une puissante séduction chez les jeunes.

Durant la rencontre du CER qui évalue ce projet, les membres expriment divers points de vue. Certains rappellent que les croustilles, les boissons gazeuses et les barres de chocolat présentent des inconvénients démontrés par rapport à la santé. Des collègues répliquent en alléguant ce qui leur semble de sens commun : ce n'est pas une consommation aussi exceptionnelle de malbouffe ${ }^{19}$ qui risque de nuire à leur santé. Puis, l'argument de l'exemplarité est invoqué. Il ne faudrait pas qu'un chercheur se comporte de manière à confirmer que la malbouffe constitue un modèle dans l'alimentation. On réplique à cet argument que, pour la plupart, les jeunes qui participeront en ont vu d'autres et ne sont pas si crédules. Enfin, un membre du CER soutient qu'offrir ces aliments, c'est miser sur une certaine faiblesse de caractère chez les jeunes approchés, abuser d'eux par un appel à la gourmandise, plutôt que de stimuler leur altruisme et leur désir de vivre en santé. Il ajoute qu'il s'agit là d'une incitation trop séduisante pour ces jeunes et conclut son laïus en citant un passage de l'EPTC2 selon lequel on doit éviter «que les participants éventuels perçoivent les incitations comme une façon de gagner des faveurs ou d'améliorer leur situation» (EPTC2, 2014, 29). Un autre membre du CER souligne que la règle à respecter est de se limiter au versement d'une indemnité compensatoire, comme le prévoit le code civil du Québec ${ }^{20}$.

Les membres des CER sont susceptibles d'adopter des postures de départ infiniment variées, censément en fonction de leurs sensibilités, de leurs valeurs et de leurs expériences. Certains sont portés à faire volontiers confiance aux équipes de recherche, alors que d'autres ne dissimulent point leur forte inclination pour la pratique du materno-paternalisme à l'égard des sujets.

19. «Nourriture malsaine en raison de sa faible valeur nutritive et de sa teneur élevée en sucres ou en gras.» (OLF, en ligne).

20. La règle de l'indemnité compensatoire apparaît à l'article 25 du Code civil du Québec:

«L'aliénation que fait une personne d'une partie ou de produits de son corps doit être gratuite; elle ne peut être répétée si elle présente un risque pour la santé.

«La participation d'une personne à une recherche susceptible de porter atteinte à son intégrité ne peut donner lieu à aucune contrepartie financière hormis le versement d'une indemnité en compensation des pertes et des contraintes subies.» 
Dans le cas de notre illustration en centre jeunesse, un chercheur ou un membre de CER peut-il légitimement présumer de la perception des sujets potentiels? Comment un chercheur ou un membre de CER animé par la bienveillance doit-il aborder une telle question? Pour celui qui adopte de bonnes dispositions à l'égard des sujets potentiels, une démarche appropriée pourrait être de poser directement la question de son point de vue au sujet ou à des personnes appartenant au même groupe que le sujet. Passant ainsi d'un point de vue présumé à la connaissance d'un point de vue exprimé, la personne animée par la bienveillance accomplirait un progrès important pour atteindre l'objectif d'une application circonstanciée des normes congruentes.

Une illustration juste de l'intention derrière cette bienveillance concerne le don d'échantillons biologiques. Le Medical Research Council (conseil) affirme que la recherche portant sur de tels échantillons devrait être conduite avec respect et transparence à l'égard des donneurs. Le conseil propose qu'avant d'approcher des donneurs potentiels les chercheurs devraient être conscients qu'il puisse y avoir des différences individuelles, culturelles ou religieuses concernant la signification et l'importance du corps humain ou de certaines de ses parties $^{21}$.

\section{Conclusion}

L'EPTC2 pose la question de savoir comment il convient de nommer quelqu'un qui contribue de sa personne à un projet de recherche. Participant ou sujet de recherche? L'EPTC2 retient participant, puisque les personnes qui choisissent de contribuer à un projet de recherche y jouent un rôle plus actif que celui qu'évoque le terme sujet.

Selon la nature de la recherche, l'intensité de la contribution du sujet peut varier considérablement. Contribuer à un essai clinique engage la personne d'une manière plus active et plus entière que confier du matériel biologique à une biobanque. Fréquemment, des chercheurs souhaitent accéder à des renseignements recueillis par diverses organisations pour d'autres finalités que la recherche. De tels projets sont réalisés dans l'ignorance totale des personnes que ces renseignements concernent. Il est alors difficile de qualifier ces sujets de participants.

Dans tout projet, celui qui contribue de sa personne à une recherche doit être traité comme un sujet au sens de l'être autonome et conscient, porteur d'une dignité inhérente. Il faut préférer sujet à participant pour trois raisons:

21. "Samples of human biological material should be treated as donations and research involving these samples should be conducted with respect and transparency.The human body and its parts should be treated with respect, and before approaching potential donors, researchers should be aware there may be individual, cultural or religious differences in the meaning and significance attached to the body or specific parts of it. Researchers should aim to achieve an environment of trust and respect with participants, recognising the altruism of providing samples for use. This environment can be fostered by maintaining a high level of transparency in every area of research practice e.g. from consent to the potential future use of samples, and dissemination of research results.» (MRC, 2014, 6) 
- l'observateur ne peut que constater l'infinie diversité des initiatives de recherche, alors que la contribution attendue de la personne varie considérablement. Parfois, la personne concernée n'entendra jamais parler du projet, parfois sa contribution au projet présentera des risques significatifs qu'elle aura préalablement acceptés. Le terme participant est peu approprié pour identifier des contributions tellement différentes,

- la définition philosophique de sujet au sens de l'être autonome et conscient rappelle au chercheur la dignité intrinsèque de celui qui contribue directement de sa personne ou par l'entremise de renseignements le concernant. Le vocable participant ne comporte pas la richesse de signification du terme sujet,

- entre les termes participant et sujet, le terme sujet - au sens philosophique de l'être autonome et conscient - est le plus susceptible d'inspirer au chercheur le respect à l'égard de celui qui contribue de sa personne à son projet de recherche.

Une règle générale issue des normes éthiques et juridiques requiert que la contribution d'un sujet à un projet de recherche soit précédée par l'expression de son consentement libre et éclairé. Cette règle s'impose, sauf pour les exceptions que la loi et les normes éthiques identifient. Suivant l'expérience commune, nous pouvons supposer que la plupart des sujets potentiels sont en mesure de comprendre les explications du chercheur et de délibérer de façon libre pour décider d'accepter ou de refuser la contribution qui leur est proposée. Néanmoins, l'histoire de la recherche sur des sujets humains permet de tirer une leçon : certaines personnes se trouvent dans une position de vulnérabilité inexorable. Parmi elles se trouvent les enfants et les personnes inaptes. À ces deux groupes, il semble opportun d'ajouter la personne en proie au désespoir, celle qui estime ne plus avoir rien à perdre, qu'il s'agisse d'elle-même ou de quelqu'un qu'elle aime.

L'aventure new-yorkaise du Dr Carrel survenue en 1908 illustre parfaitement un consentement qu'expriment des parents habités par le désespoir. L'intervention tentée ne se situait pas dans l'environnement scientifique et éthique qui encadre aujourd'hui la recherche. Toutefois, cet événement révèle comment des personnes plus informées que la moyenne des gens osent envisager un recours dont la pulsion est l'énergie du désespoir. Si le bébé survit, l'intervention aura prolongé une existence humaine et épargné aux parents le drame de perdre leur enfant, du moins à court terme.

Partant de cette conscience du désespoir, observons comment d'autres contextes poignants nous renseignent sur les motivations des sujets de recherche : la littérature médicale fournit des témoignages à ce sujet. Le professeur américain de psychiatrie Paul S. Appelbaum a publié de nombreux articles sur la notion de méprise thérapeutique. Une de ces publications, datant de 1987, décrit comment un patient conçoit sa contribution à la recherche en psychiatrie. Selon toute apparence, le consentement exprimé est libre et éclairé. Cependant, quand on demande au patient pourquoi il a consenti à la recherche, il répond que la médication qu'il recevra sera celle qui peut le mieux améliorer sa condition, manifestant ainsi une méprise thérapeutique.

Deux attitudes du chercheur et de son équipe peuvent contribuer à la possibilité, pour le sujet potentiel, de participer à la recherche en exprimant une volonté libre et éclairée : l’objectivité et la bienveillance.

Le chercheur soucieux d'objectivité s'adresse au sujet potentiel en recourant à la persuasion plutôt qu'à la séduction. La persuasion sollicite l'intelligence d'une personne : elle utilise une argumentation rationnelle dans l'intention de la convaincre. La séduction lorgne du côté des émotions, de la vie psychique. Dans sa personnification la plus terrible, le séducteur n’hésite nullement à «exercer sur quelqu'un tous les moyens de plaire, en particulier pour l'abuser, faire illusion, le faire agir dans un sens bien précis» (Larousse, 1997, 6680). Au fil de la vie quotidienne, chacun constate que la beauté et l'argent exercent un pouvoir de séduction. Dans l'univers de 
la recherche, il est possible pour le chercheur d'employer un discours séducteur, un boniment, qui enchante par les nuances agréables et prometteuses, mais qui néglige de présenter avec objectivité ou franchise les risques et les inconvénients d'un projet.

La bienveillance est l'attitude de la personne qui montre de bonnes dispositions à l'égard de quelqu'un. Est-il possible, dans la réalisation d'un projet, de tenir compte de la personnalité du sujet, de son point de vue, concernant les avantages, les risques ou les inconvénients que comporte la recherche? Une première démarche appropriée pourrait être de poser directement la question de son point de vue au sujet.

L'objectivité et la bienveillance fournissent une inspiration éthique au chercheur, elles bonifient sa pratique professionnelle, et leur exercice constitue une sauvegarde pour les sujets de recherche. 


\section{Bibliographie}

Alain. (1970). Propos, Tome 2, Pléiade, no 619.

Appelbaum, P.S., Roth, L.H., Lidz, C.W., Benson, P. et Winslade, W. False Hopes. (1987). Best Data : Consent to Research and the Therapeutic Misconception. Hastings Center Report, 1987, 17(2) : 20-4.

Beauchamp T.L. et Childress J.F. (2103). Principles of Biomedical Ethics, $7^{e}$ éd., Oxford University Press.

Blais, M. (1984). Une morale de la responsabilité, Fides.

Canadian Medical association (CMA). (2008). Research Guidelines : a web-based decision guide for physicians. Repéré à https://www.cma.ca/Assets/assets-library/document/en/Final_Toolkit_Research-e.pdf

Charte des droits et libertés de la personne (Charte), RLRQ, c. C -12.

Code civil du Québec (CCQ), RLRQ, c. CCQ-1991.

Code de déontologie des médecins, RLRQ, c. M -9, r. 17.

Le Robert. (1998). Dictionnaire historique de la langue française, Paris.

Dorval J. et Hébert B. (2005). Quelques balises éthiques pour composer avec le sort incertain des extrêmes prématurés. Dans Michel T. Giroux et al., L'extrême prématurité. Les enjeux parentaux, éthiques et légaux, (59-68). PUQ.

Conseil de recherches en sciences humaines du Canada, Conseil de recherches en sciences naturelles et en génie du Canada, Instituts de recherche en santé du Canada (décembre 2014). Énoncé de politique des trois Conseils : Éthique de la recherche avec des êtres humains. (cité dans le texte comme EPTC2) http://www.ger.ethique.gc.ca/pdf/fra/eptc2-2014/EPTC_2_FINALE_Web.pdf.

Giroux, M.T. (2010). Cultiver des attentes pragmatiques à l'égard des comités d'éthique». Dans Trudel P. et S.Jean, M. (ed.) La malréglementation. Une éthique de la recherche est-elle possible et à quelles conditions? (113-128) PUM.

Larousse. (2003). Grand dictionnaire de la philosophie. Paris

Larousse. (1997). Grand Usuel Larousse, dictionnaire encyclopédique, Paris.

Kalanithi, P. (2016). When Breath Becomes Air, New York: Random House, New Yok.

Loi médicale, RLRQ, c. M-9.

Loi sur les servies des santé et les services sociaux (LSSSS), RLRQ, c. S-4.2.

Marina, J.A. (2004). La inteligencia fracasada. Teoria y práctica de la estupidez. Editorial Anagrama.

Medical Research Council (MRC). (2014, novembre). Human Tissue and Biological Samples for Use in Research: Operational and Ethical Guidelines, MRC Ethics Series.

Office de la langue française (OLF), Grand dictionnaire terminologique.

Organisation des Nations Unies pour l'éducation, la science et la culture (UNESCO). (2005). Déclaration universelle sur la bioéthique et les droits de l'homme.

Oxford. English Oxford Living Dictionaries. Repéré à https://en.oxforddictionaries.com/

Starr, D. (2002). Blood; An Epic History of Medicine and Commerce, Harper. 
TITRE: LITTÉRATIE ET CONSENTEMENT ÉCLAIRÉ À LA RECHERCHE : ÉTAT DES LIEUX ET PISTES DE SOLUTION

Auteur(s): Ana Marin et Michel T. Giroux

Publication: RECRUTEMENT ET CONSENTEMENT À LA RECHERCHE : RÉALITÉS ET DÉFIS ÉTHIQUES

PAGES: $27-38$

ISBN: 978-2-7622-0357-8

Directeurs: AnA MARIN, BÉATRICE EYSERMANN ET MiCHEL T. GIROUX

URI: HTTP://HDL.HANDLE.NET/11143/14105

DOI: HTTPS://DOI.ORG/10.17118/11143/14105 


\section{Littératie et consentement éclairé à la recherche : état des lieux et pistes de solution}

Ana Marin, professeure associée Université de Sherbrooke, membre des Comités d'éthique de la recherche du CHU de QuébecUniversité Laval et du CISSS Chaudière-Appalaches, membre de l'Institut de consultation et de recherche en éthique et en droit

(ICRED) et membre associée de l'IDEA.

Michel T. Giroux, avocat et docteur en philosophie, directeur de l'Institut de consultation et de recherche en éthique et en droit (ICRED), président du Comité d'éthique de la recherche des établissements du Centre de recherche interdisciplinaire en réadaptation du Montréal métropolitain (CRIR), président du Comité d’éthique de la recherche Biobanque Genizon chez Génome Québec, professeur associé au département de médecine sociale et préventive à la Faculté de médecine de l'Université Laval.

Résumé : Les statistiques montrent qu’environ la moitié de la population québécoise et canadienne a un niveau de littératie en santé qui ne lui permet pas toujours de formuler des décisions éclairées concernant sa propre santé. Qu'en est-il de la recherche et du caractère éclairé du consentement dans ces circonstances? La question est analysée à travers la définition de la littératie en santé développée par l'ICRED au cours de ses travaux des dernières années.

Mots-clés : littératie en santé, consentement éclairé, consentement à la recherche, éthique, ICRED. 


\section{Introduction}

Des données d'enquêtes internationales montrent que plus de la moitié des Québécois et des Canadiens ne disposent pas du niveau nécessaire de littératie en santé pour bénéficier pleinement du réseau de la santé et des services sociaux. Ce constat pose inévitablement plusieurs questions d'ordre éthique, notamment en lien avec l'exercice de l'autonomie et l'accès aux ressources disponibles. Et si nous considérons le niveau de littératie du sujet ${ }^{1}$ de recherche, comment devrions-nous nous y prendre pour que son consentement soit véritable, à savoir libre et éclairé?

Cet article débute par une présentation des enjeux de santé et des enjeux éthiques que pose la littératie en santé. Notre point de départ est la définition de la littératie en santé issue des travaux de l'Institut de consultation et de recherche en éthique et en droit (ICRED). Puisque le faible niveau de littératie en santé nous questionne sur la qualité du consentement à la recherche, nous poursuivons avec des données issues d'une revue de littérature au sujet de la compréhension de la recherche par les participants, notamment dans le processus d'obtention du consentement. Enfin, des pistes de solutions sont proposées en vue de favoriser le caractère éclairé du consentement à la recherche.

\section{Littératie en santé : définition de l'ICRED}

Les préoccupations concernant la littératie en santé sont issues d'une enquête internationale réalisée en 2003 et reprise en 2013 qui montrait qu'au Canada et au Québec les taux de littératie révèlent qu'environ la moitié de la population ne détient pas les compétences nécessaires pour fonctionner avec aisance dans la société actuelle. Au cours de leurs rencontres de travail avec diverses personnes préoccupées par la littératie, les membres de l'ICRED ont constaté que les résultats de ces deux enquêtes ont particulièrement attiré l'attention des milieux d'éducation, des pharmaciens et des professionnels de la santé publique.

Lorsqu'il est question de littératie en général, et de littératie en santé en particulier, plusieurs définitions sont vraisemblables. Afin que notre propos soit aussi clair que possible, il importe de commencer en proposant une définition de la littératie en santé. Évidemment, l'Agence de la santé publique du Canada (Agence) s'intéresse à littératie en santé, puisque ce facteur intervient de façon prépondérante dans l'état de santé des Canadiens. L'Agence retient cette définition : la «capacité de trouver, de comprendre, d'évaluer et de communiquer l'information de manière à promouvoir, à maintenir et à améliorer sa santé dans divers milieux au cours de sa vie» (2018). Selon cette définition, la littératie apparaît d’abord comme une capacité. Néanmoins, il nous semble qu'un élément important est absent de cette définition, soit la capacité de reconnaître l'existence d'un besoin d'information.

L'équipe de l'ICRED a travaillé de 2010 à 2014 sur la question de la littératie en santé dans une perspective éthique, notamment en lien avec l'exercice de l'autonomie et la pratique de la justice dans l'accès aux services². En effet, la problématique s'est posée ainsi : le système de santé est-il juste s'il y a une discordance entre le

1. Le terme «sujet de recherche» est utilisé dans cet article au lieu de «participant à la recherche», voir Giroux dans ce collectif.

2. Les travaux de l'ICRED ont pris la forme de revues de la littérature, de rencontres avec des experts, d'échanges et de réflexions en groupe. Ces démarches ont permis au groupe de mettre au point une définition de la littératie. À terme, les travaux de l'ICRED ont donné lieu à un colloque sur la littératie en santé où plusieurs conférenciers ont présenté une réflexion sur le sujet en présentant leur domaine de pratique (par exemple, en soins intensifs, en génétique, en santé publique) et en mettant en évidence l'élément ou les éléments de la littératie sur lesquels il est possible d'intervenir pour réduire l'écart entre le langage du système de la santé et les compétences des usagers. En ligne: www.instituticred.org 
langage utilisé par le système et le niveau de littératie de ses usagers, si pour certaines personnes l'accès aux soins et aux services est plus difficile que pour d'autres en raison de leur capacité moindre à en comprendre le fonctionnement? D’autre part, peut-on vraiment considérer que les usagers sont en mesure d'exercer leur autonomie étant donné les limites imposées par cette discordance?

La définition de la littératie que l'équipe de l'ICRED a retenue correspond au souci de formuler cette capacité d'une façon aussi complète que possible, depuis la perception d'un besoin d'information jusqu'à la prise d'une décision éclairée. Au terme de multiples échanges, l'ICRED a adhéré à cette définition de la littératie:

Capacité de reconnaître l'existence d'un besoin d'information en matière de santé ou de services de santé, de déterminer l'information nécessaire, de la trouver, de la comprendre et de la traiter en vue de prendre une décision éclairée.

Il faut réaliser que notre définition comporte un certain niveau d'exigence. La littératie est définie comme une capacité, c'est-à-dire une aptitude «dans tel ou tel domaine» (Larousse, 1997 : 1161), ici, celui de la santé. Cette capacité a pour objet une prise de conscience concernant un besoin d'information, une information qu'il s'agit de trouver, de traiter et de communiquer. L'objectif est de favoriser sa propre santé dans diverses circonstances, tout au long de sa vie. Si nous supposons que toute personne subira la maladie, de manière parfois sérieuse, de même que des revers et des déconvenues, il est probable qu'elle se trouvera parfois dans un état qui diminue plus ou moins considérablement son niveau de littératie en santé. On pense ici à la vulnérabilité que la maladie apporte dans la vie d'une personne, par exemple certaines maladies peuvent altérer le jugement de la personne, son état cognitif général. L'annonce, à elle seule, d'un diagnostic grave peut influencer l'état psychologique et émotif de la personne. Et même, le simple fait de se retrouver dans des milieux impersonnels, avec leurs règles de fonctionnement propres, comme les hôpitaux, les cliniques, peut causer des appréhensions.

\section{Littératie en santé et en recherche : enjeu quotidien}

La littératie en santé se présente comme un déterminant de la santé (Hoffman-Goetz et coll., 2014 : 61-78) au même titre que le revenu et le statut social, le réseau de soutien social, l'éducation et l'alphabétisme, l'emploi et les conditions de travail, l'environnement social, l'environnement physique, les pratiques de santé personnelles, le développement sain de l'enfant, le patrimoine biologique et génétique, les services de santé, le genre et la culture.

L'Agence publie certains chiffres concernant les aptitudes des adultes et des personnes âgées en relation avec la littératie en santé :

Les chiffres démontrent que $60 \%$ des adultes et $88 \%$ des personnes âgées au Canada n’ont pas les compétences nécessaires pour trouver, comprendre et utiliser l'information de manière à pouvoir prendre de bonnes décisions pour leur santé. Les personnes qui affichent un faible niveau de littératie en santé ont de la difficulté à comprendre et utiliser l'information sur la santé à laquelle ils sont exposés tous les jours, de l'information couramment disponible dans les établissements de soins de santé, les épiceries, les points de vente au détail, les écoles, les médias ainsi qu'au sein de leur communauté. (2018)

Les chiffres que présente l'Agence sont révélateurs d'importantes carences chez les adultes et les personnes âgées. Néanmoins, l'information portant sur la santé est disponible quotidiennement, et ce, dans plusieurs milieux, ajoute l'Agence. Cette observation suggère que la communication efficace de l'information en matière de santé nécessite un contact soutenu entre la personne qui communique l'information et celle qui la reçoit. 
La recherche en santé constitue un domaine différent de celui des services de santé et des services sociaux. Par contre, ces deux domaines ont pour caractéristique commune de se rapporter à la santé, donc de contenir inévitablement les éléments de complexité propres au monde de la santé. Le patient du système de santé devient, de par sa condition de santé, un sujet de recherche potentiel. À la complexité du monde de la santé s'ajoute aussi la complexité du monde de la recherche. Pensons seulement à tout le vocabulaire des essais cliniques (randomisation, double aveugle, placébo, etc.), à celui de la recherche en génétique ou encore en imagerie et autres technologies médicales. Et cette complexité reliée au monde de la recherche nous laisse croire que les enjeux en lien avec la littératie des sujets de recherche sont présents dans d'autres recherches que celles réalisées en santé. Pensons, par exemple, au langage utilisé pour présenter la confidentialité des données de recherche (codifiées, anonymes, rendues anonymes, clé du code, etc.)

Le sujet éventuel d'un projet de recherche reçoit des explications des membres de l'équipe de recherche et il dispose du formulaire d'information et de consentement (FIC) qui intervient à la fois comme document explicatif et comme contrat. Dans cette circonstance, le niveau de littératie intervient comme déterminant d'une participation plus ou moins renseignée et éclairée.

\section{Littératie, autonomie et justice}

Ce que nous constatons à propos de la littératie engendre des préoccupations en matière d'éthique. Deux principes bien connus de l'éthique médicale orientent cette réflexion : le respect de l'autonomie et la justice.

D’après un dictionnaire encyclopédique, une personne autonome est «quelqu'un qui a une certaine indépendance, qui est capable d'agir sans avoir recours à autrui» (Larousse, 1997 : 579). L'essence de cette définition n'exprime pas l'idée qu'une personne autonome est celle qui ne tient jamais compte de l'existence d'autrui, mais qu'elle peut prendre des décisions sans devoir suivre l'avis ou les préférences d'une autre personne. Nous ne déployons pas notre autonomie dans la vacuité; nous nous trouvons en relation avec de nombreuses personnes dont l'influence est plus ou moins marquée.

L'autonomie ne s'exerce pas dans le vide; au contraire, elle est soumise à l'influence des diverses relations entre la personne et sa famille, son milieu et les groupes auxquels elle appartient, que ces liens soient d'ordre culturel, social, linguistique, religieux ou autre. (EPTC2, 2014 : 7)

En accord avec cette description de la personne autonome, «l'autonomie désigne la capacité de se diriger d'après sa propre volonté. Ainsi, selon le principe d'autonomie, chaque personne est maîtresse d'elle-même et décide de ce qui lui convient» (Giroux, 2005 : 45). Appliquée au contexte de la relation entre un chercheur et un sujet éventuel, cette définition de l'autonomie suppose que les explications du chercheur permettent une décision éclairée, puisque le sujet éventuel détient alors l'information requise pour parvenir à une décision prise selon, justement, «ce qui lui convient».

L'autonomie sous-entend la capacité de définir ses objectifs personnels et la liberté d'agir en conséquence (Roy et coll, 1995 : 123). Une condition intrinsèque à l'exercice de l'autonomie est l'accès à l'information pertinente pour la prise de décision. Et, dans le cas d'un niveau bas de littératie, par rapport au niveau du langage des intervenants de la santé ainsi que du système de santé, l'exercice de l'autonomie ne devient-il pas un mirage? On ne peut plus tenir un discours sur l'importance du respect de l'autonomie des usagers du système de santé et des sujets de recherche sans se soucier des conditions dans lesquelles l'autonomie est censée s'exercer en 
réalité. Nous devons vérifier la réalisation concrète d'une des caractéristiques fondamentales d'un consentement véritable, à savoir son caractère éclairé.

Le terme justice comporte quant à lui de nombreuses définitions. Par exemple, la justice peut désigner l'ensemble des autorités chargées d'appliquer le droit. Dans le présent contexte, nous utilisons justice au sens du «principe moral qui exige le respect du droit et de l'équité» (Larousse, 1997 : 4072). Considérée comme un principe moral, la justice peut par exemple être générale ou particulière, commutative ou distributive. Puisque notre préoccupation consiste dans l'accès aux services de santé et à la recherche, la justice distributive fait l'objet de notre attention:

La justice appelée distributive a pour objet la répartition du bien commun. Elle fixe un partage proportionné et un usage adéquat des ressources. Elle traite ce qui est égal également et ce qui est inégal inégalement (EPTC2, 2014 : 7).

Lorsque la personne qui nécessite des ressources du système de santé comprend son besoin, est capable de l'exprimer et sait à qui s'adresser, elle peut recevoir les soins que nécessite son bien-être. Cette personne bénéficie de la justice distributive en matière de santé. Par contre, lorsque la personne qui requiert des soins évalue mal son besoin, est peu capable de l'exprimer et ne sait pas à qui s'adresser, il est probable qu'elle ne recevra pas les interventions appropriées. Cette personne ne bénéficie pas de la justice distributive en matière de santé.

Vue dans une perspective d'accès aux services de santé, la littératie apparaît comme une condition à l'exercice de ce droit (Larousse, 1997 : 1161). Reconnu par l'OMS comme un droit fondamental de chaque personne, le droit à la santé est inscrit dans la réglementation internationale. Notons que le droit à la santé ne veut pas dire que tout le monde doit être en santé (Hoffman-Goetz , 2014 : 11), mais que les systèmes de santé doivent être accessibles à tous, sans aucune discrimination. Ainsi, la loi du Québec n'affirme pas l'existence d'un droit à la santé, mais plutôt celle d'un droit d'accès aux services de santé : « Toute personne a le droit de recevoir des services de santé et des services sociaux adéquats sur les plans à la fois scientifique, humain et social, avec continuité et de façon personnalisée et sécuritaire » (LSSSS, art 5).

Rappelons que les enquêtes populationnelles observent des liens de plus en plus clairs entre le niveau de littératie et l'état de santé des personnes. Inévitablement, nous devons nous poser la question sur l'accès aux soins et aux services de santé en termes de justice. Pouvons-nous considérer que notre système de santé est juste à l'égard de tout citoyen? Ce système est-il véritablement accessible dans un contexte où plus de la moitié de la population éprouve de la difficulté à y accéder? Le niveau de langage des professionnels de la santé et des organisations est-il adapté au niveau de littératie des divers groupes de citoyens? Est-il acceptable qu'on adresse le même message à toute la population alors que les écarts au niveau de la capacité à comprendre et à agir dans le système de santé sont si considérables? 


\section{Dignité et consentement à la recherche}

À la lumière de ce qui vient d'être énoncé, on est légitimé de se questionner sur la qualité du consentement à la recherche, notamment sur le respect de l'exigence qu'il soit éclairé. Selon l'Énoncé de politique des trois Conseils (EPTC2), le caractère volontaire du consentement ne se trouve en relation avec rien de moins que la dignité humaine :

Le caractère volontaire du consentement est important. En effet, il s'accorde avec le respect de la dignité humaine et signifie que la personne a choisi de participer en fonction de ses valeurs, de ses préférences et de ses désirs (2014: 28).

Pour que la personne exerce un choix en fonction de ses valeurs, il est essentiel qu'elle comprenne au moins la généralité de ce qui lui est proposé. Il ne s'agit pas seulement de recevoir l'information, mais de la traiter et de l'évaluer en fonction de ses valeurs. Or, puisque l'équipe de recherche détient le savoir scientifique et que c'est elle qui sollicite la participation de sujets éventuels, il lui revient d'expliquer son projet de manière à générer la compréhension de la personne approchée. C'est pourquoi l'EPTC2 affirme :

Les chercheurs doivent divulguer aux participants éventuels ou aux tiers autorisés tous les renseignements pertinents leur permettant de prendre une décision éclairée relativement à leur participation au projet de recherche (2014: 30$)$.

Usuellement, la transmission de l'information du chercheur au sujet potentiel est réalisée par des échanges verbaux et par l'écrit, principalement le texte du formulaire de consentement. Toutefois, d'autres moyens de communication peuvent être utilisés : le dessin, le graphique, les instruments technologiques comme les moyens audio ou visuels (Côté et coll. 2018).

Pour qu'il y ait consentement éclairé, les participants éventuels doivent comprendre l'information que les chercheurs leur transmettent. Les chercheurs et les CÉR réfléchiront à la meilleure façon de communiquer cette information afin d'en faciliter la compréhension. Par exemple, la documentation écrite peut être accompagnée de moyens audio ou visuels ou de présentations vidéo (EPTC2, 2014 : 32).

La finalité de cette diversité dans les moyens est de communiquer efficacement avec le futur sujet de recherche.

\section{La compréhension des participants à la recherche : état des lieux}

Pour prendre connaissance de la situation quant au niveau de compréhension en contexte de recherche, les auteurs du texte ont procédé à une revue de littérature. Quatre types de documents ressortent:

- revues de littérature;

- réflexions théoriques;

- évaluations de processus de consentement, souvent par un questionnaire ou une entrevue tout de suite après l'obtention du consentement afin de valider la compréhension de l'information transmise par l'équipe de recherche;

- description d'outils d'évaluation du niveau de littératie (ex. : REALM (Nishimura et coll., 2013 : 28). 
Parmi les revues de littérature, deux de type systématique ont retenu notre attention par leur pertinence en lien avec le sujet discuté dans cet article.

La première avait recensé 54 interventions testées dans des essais randomisés. Leurs auteurs ont catégorisé trois sortes d'intervention : multimédias, formulaires de consentement améliorés (rendus plus lisibles ; brièveté, simplicité, apparence du formulaire, etc.) et discussions étendues («focus group», «standardized discussion», conversations téléphoniques ultérieures, etc.). Les résultats de ces études montrent que les formulaires de consentement améliorés et les discussions étendues sont les plus efficaces (Nishimura et coll., 2013 : 28).

La deuxième revue systématique a recensé des études qui visaient particulièrement les populations avec un faible niveau de littératie. Six études ont été retenues. Elles regroupent 1620 participants : personnes âgées, minorités ethniques et niveau de littératie correspondant à moins de 8 années de scolarité. La conclusion retenue est que la discussion un à un est la stratégie la plus efficace (Tamariz L et coll., 2013).

Une mauvaise compréhension ou une compréhension faible des renseignements concernant une recherche, dans le cas des essais cliniques notamment, produit ce qu'on appelle une méprise thérapeutique. Paul Appelbaum et son équipe ont beaucoup étudié ce phénomène. Lors d'une conférence en éthique de la recherche (PRIMW\&R, 2013), Appelbaum a rapporté l'exemple, issu de ses recherches, d'une participante à un essai clinique randomisé. Lors de son entrevue avec ce chercheur, elle montre avoir retenu qu'il s'agissait d'un essai clinique randomisé - elle a même su donner la définition du concept de randomisation! En même temps, cette personne croyait qu'elle recevrait le meilleur des deux traitements. C'est comme si le lien entre la connaissance (la définition de randomisation) et son espoir (avoir le meilleur traitement) ne s'était pas fait. Dans un autre cas, un sujet a compris qu'il aurait le traitement standard dans un bras et le traitement expérimental dans l'autre, alors que le concept de bras était utilisé par l'équipe de recherche pour distinguer les groupes en fonction de la répartition aléatoire des traitements... Un mot qui a une signification universellement connue au sens propre, le bras comme partie du corps, a été utilisé au figuré, bras de l'étude, et cette utilisation du mot a pu induire en erreur la dame concernée.

Selon Appelbaum, ce qui pose problème, entre autres, c'est le langage utilisé. On préfère les mots comme «étude», «nouveau médicament», «nouveau traitement» ou «nouvelle thérapie» au lieu, respectivement, de «recherche», «médicament expérimental» ou «traitement expérimental», «thérapie expérimentale». À cela s'ajoute le double chapeau du médecin chercheur : lorsque le médecin traitant propose une recherche à ses patients, il y a de fortes chances que ceux-ci supposent qu'il s'agit du meilleur traitement possible dans leur cas. Appelbaum attire aussi l'attention sur un autre phénomène qui peut influencer la prise de décision, à savoir les acronymes d'études ayant une connotation thérapeutique enthousiaste : BEST, MAGIC, MIRACL, PROVED, PROSPER...

La revue de littérature sur la compréhension dans le processus de consentement à la recherche nous a permis de mettre en évidence les éléments suivants comme barrières à une bonne compréhension :

- l'utilisation d'un jargon médical, d'un langage trop technique pour les sujets de recherche;

- l'abstraction des risques due à la manière dont ils sont présentés (\%, x sur 100, etc.) et donc la difficulté, pour un sujet de recherche, de bien se les représenter - il en découle une différence de perception notable entre lui et le professionnel;

- l'impact négatif des conditions de santé du sujet sur son état cognitif et donc sur son niveau de littératie - dans un tel cas, la compréhension n'est pas liée au seul niveau de scolarité; 
- la réticence plus grande des personnes ayant un faible niveau de littératie à poser des questions, ce qui les rend davantage malléables;

- la discordance entre la forme du message et le niveau de littératie du destinataire - les personnes ayant un faible niveau de littératie préfèrent l'information verbale, alors que les personnes ayant un niveau élevé de littératie optent pour l'information écrite;

- la confiance que le sujet éprouve envers le clinicien, le chercheur ou l'institution apparaît comme un élément déterminant dans sa tendance à ne pas souhaiter comprendre véritablement - plus on a confiance, plus on se fierait aux explications verbales.

De plus, la nature des recherches étant en constant changement, et les domaines étudiés se diversifiant continuellement, le consentement tel qu'il est conçu actuellement semble bien souvent inadéquat. C'est notamment le cas s'agissant de bases de données et des croisements possibles entre les données venant de différentes sources (FRSQ, 2006 : 62). Les participants consentent-ils de manière éclairée à la mise en banque de leurs données et de leur matériel biologique? Sont-ils suffisamment éclairés sur l'utilisation possible, technologiquement parlant, de leurs données provenant de différents moteurs de recherche, de logiciels ou des témoins?

\section{Améliorer le caractère éclairé du consentement}

Les travaux de l'ICRED ont également permis de constater qu'il y a deux façons d'envisager une amélioration du niveau de littératie. Premièrement, partant de la capacité de l'usager, la préoccupation à l'égard du niveau de littératie pose aux professionnels et aux établissements le défi de fournir une information accessible à l'usager ou au public visé. Un accès aux services respectueux de l'autonomie requiert que les professionnels adoptent des approches leur permettant d'être compris. Les demandes du système de santé, mais aussi sa complexité, doivent faire l'objet de changements «to better align care demands with the public's skills and abilities» (French et Hernandez, 2013: 1).

Deuxièmement, il s'agit de travailler au rehaussement du niveau de littératie des individus et de la collectivité, ce qui se travaille actuellement surtout du côté de la santé publique. Dans ce sens, le directeur de santé publique du CIUSSS du Centre-Sud-l'île-de-Montréal, M. Richard Massé, affirme en introduction d'un guide sur la littératie en santé :

Si des efforts doivent être entrepris pour améliorer le niveau de littératie de l'ensemble de la population - particulièrement les populations défavorisées - il importe également d'adapter les interventions existantes et futures pour répondre aux enjeux de faible littératie en santé (Lemieux, 2014 : iii).

Le Conseil canadien sur l'apprentissage indique à son tour :

Les praticiens et chercheurs s'entendent sur le fait que la littératie en santé est le fruit de l'interaction entre les capacités des personnes et les demandes du système de santé. Voilà pourquoi on cherche à améliorer la littératie en santé en haussant le niveau de littératie des adultes et en abaissant les demandes du système de santé en matière de littératie. (CCA, 2007 : 11)

Si on se tourne vers la recherche maintenant, est-il possible de travailler sur ces deux aspects? 
Il nous semble envisageable, et même nécessaire de travailler de façon plus globale pour rehausser le niveau de littératie des sujets de recherche, qu'ils soient actuels ou purement potentiels. Dans ce sens, des activités de vulgarisation, autant sur la science en général que plus spécifiquement sur la recherche scientifique, permettraient à la population en général d'avoir des connaissances de base dans ces domaines et ainsi d'être mieux prête à accueillir des informations plus détaillées sur un projet de recherche en particulier.

Cette responsabilité d'éduquer la population en matière scientifique revient aux instances gouvernementales, aux universités et aux autres institutions d'enseignement et de recherche. En plus de la vulgarisation de la recherche, comme cela se fait par exemple lors des congrès annuels de l'ACFAS, il faudrait vulgariser le processus de recherche, montrer ses possibilités, exposer les enjeux éthiques qu'il soulève et identifier ses limites, et ce, par des émissions de vulgarisation à la télé, à la radio, sur le web ou d'autres moyens. Reconnaissons cependant qu'il s'agit là d'un vaste chantier qui pourrait mettre des années à se réaliser.

D'un autre côté, il est nécessaire, plus particulièrement lors de la préparation d'un nouveau projet de recherche, de rédiger la documentation de manière à ce qu'elle soit facilement compréhensible pour l'ensemble des sujets éventuels, avec des outils d'adaptation permettant à tout un chacun de comprendre les implications de leur participation au projet de recherche proposé. Cette approche permettrait d'être plus équitable dans le recrutement et dans le processus d'obtention du consentement.

\section{Conclusion}

Après avoir précisé la définition du concept de littératie utilisée dans cet article, nous avons bâti notre réflexion à partir du constat suivant : il y a une discordance significative entre, d'un côté, le niveau de littératie en santé et en recherche de la population canadienne et québécoise et, d'un autre côté, la haute complexité du système de santé et celui de la recherche. Sur le plan éthique, cette discordance nous a amenés à questionner les limites que cela pose aux sujets de recherche potentiels dans l'exercice de leur autonomie et aussi dans le respect du principe de justice.

En effet, pour qu'une personne puisse prendre une décision selon « ce qui lui convient » - c'est-à-dire exercer son autonomie - elle doit avoir l'information pertinente. Il faut aussi que cette information soit claire et compréhensible pour elle. Pour que le processus de consentement soit une véritable expression du principe de respect de l'autonomie, il doit inévitablement tenir compte du niveau de littératie du sujet de recherche potentiel.

La justice est l'autre principe à considérer en relation avec un bas niveau de littératie chez le sujet de recherche. En effet, puisque la moitié de la population canadienne et québécoise ne semble pas avoir le niveau nécessaire de littératie - cette capacité de reconnaître l'existence d'un besoin d'information, de déterminer l'information nécessaire, de la trouver, de la comprendre et de la traiter - leur accès à la santé et à la recherche peut être limité. Cela impliquerait pour la recherche, entre autres, un déséquilibre entre ceux qui portent le poids des risques (pensons aux sujets qui acceptent la participation à un essai clinique par méprise thérapeutique) et ceux qui profitent des résultats de la recherche, ou encore, le défi de la représentativité dans les résultats (si des sujets potentiels n'y participent pas par méconnaissance de la recherche). Afin de respecter ce principe à la base de l'éthique de la recherche, le processus du consentement ne peut pas se faire sans souci pour la compréhension que la personne a de la recherche qu'on lui propose. 
Pour diminuer le plus possible l'écart entre le niveau de littératie de la personne approchée pour participer à une recherche et la complexité de la recherche elle-même, il est important d'envisager des actions bidirectionnelles. D'un côté simplifier le langage de la recherche et, de l'autre, travailler à augmenter le niveau de littératie de la population en matière de recherche.

Il ne faut pas oublier que dans le processus de consentement, le sujet de recherche potentiel est dépendant de l'équipe de recherche. Et ce facteur de dépendance devient plus déterminant lorsque le niveau de littératie de la personne ne lui permet pas de comprendre avec une relative facilité les explications qui lui sont données. Dans ces circonstances, le rôle pédagogique de l'équipe de recherche est amplifié par son obligation de fournir des informations que comprend ce sujet, cette personne en particulier, et de s'assurer de sa compréhension. Afin d'établir une communication aussi efficace que possible, l'équipe de recherche doit prendre les moyens pour connaître les sujets potentiels, possiblement lorsqu'elle les rencontre individuellement. Il arrive aussi que le thème de la recherche permette de repérer le terrain de réalisation du projet ou d'identifier une population précise. L'équipe de recherche peut alors connaître les grandes caractéristiques de la population visée et lui offrir une communication appropriée.

Le FIC est un des outils dans le processus de communication qui conduit au consentement. Celui-ci doit être adapté le plus possible au niveau de littératie du sujet. De plus, les données de la littérature montrent que d'autres moyens de communication et surtout les conversations en personne aident à augmenter la compréhension qu'a le sujet des implications de sa participation, de manière à ce que la volonté qu'il exprime soit véritablement libre et éclairée.

L'absence d'une attitude sensible aux sujets potentiels chez l'équipe de recherche situe obligatoirement les chercheurs dans une approche où l'information considérée nécessaire pour une décision éclairée est produite selon un modèle préétabli ne pouvant pas être adapté aux caractéristiques et aux besoins particuliers.

Le véritable processus de recrutement et de consentement s'accorde avec le respect de la dignité humaine. Pour que la personne puisse faire un choix en fonction de ses valeurs, de ses préférences et de ses désirs, il faut maintenir le cap vers la compréhension réelle, pleine et entière, par la personne sujette à la recherche, de toutes les implications de sa participation, et ce, au-delà de toutes les règles légales et des normes administratives. Cette compréhension réelle dépend largement du niveau de littératie du sujet. C'est pourquoi le langage utilisé auprès des sujets éventuels doit être accessible, voire adapté, à chaque personne approchée. 


\section{Bibliographie}

Agence de la santé publique du Canada (Agence). (2018). Littératie en santé. http://www.phac-aspc.gc.ca/cd$\mathrm{mc} / \mathrm{hl}$-ls/index-fra.php

Conseil canadien sur l'apprentissage (CCA). (2007). Littératie en santé au Canada : Résultats initiaux de l'Enquête internationale sur l'alphabétisation et les compétences des adultes. Ottawa.

Conseil de recherches en sciences humaines du Canada, Conseil de recherches en sciences naturelles et en génie du Canada, Instituts de recherche en santé du Canada (décembre 2014). Énoncé de politique des trois Conseils : Éthique de la recherche avec des êtres humains. (cité dans le texte comme EPTC2) http:// www.ger.ethique.gc.ca/pdf/fra/eptc2-2014/EPTC_2_FINALE_Web.pdf.

Côté, I., Trottier-Cyr, R-P., Lavoie, K. et Pagé G. (2018). «Veux-tu participer à ma recherche? : principes, enjeux et stratégies concernant l'assentiment des enfants dans le processus de recherche. Dans : Marin, A., Eysermann, B. et Giroux M.T. (2018). Recrutement et consentement en recherche: réalités et défis éthiques. Sherbrooke: EDUS.

Fonds de la recherche en santé du Québec (FRSQ). (2006). Rapport final du groupe-conseil sur l'encadrement des banques de données et des banques de matériel biologique à des fins de recherche en santé, Montréal.

French, M., et Hernandez, L. M. (2013). Introduction. Organizational change to improve health literacy: workshop summary. National Academies Press.

Giroux Michel T. (2005). Au cœur de la consultation médicale : la délibération sur un cas d’éthique clinique. Le Médecin du Québec, 40 (1).

Gouvernement du Québec. Loi sur les services de santé et les services sociaux. S-4.2 (cité dans le document comme LSSSS)

Larousse. (1997). Grand Usuel Larousse, dictionnaire encyclopédique, Paris.

Hoffman-Goetz, L., Donelle, L. et Rukhsana A. (2014). Health Literacy in Canada : A Primer for Students. Canadain Scholar's Press Inc.

Lemieux, V. (2014). Pour qu’on se comprenne. Guide de littératie en santé, Montréal : ASSSM.

Nishimura, A., Carey, J., Erwin, P.J., Thilburt J.C., Murad, M.H., McCormik J.B. (2013). Improving understanding in the research informed consent process: a systematic review of 54 interventions tested in randomized control trials. BMC Medical Ethics, 14: 28.

Roy, D.J., Williams, J.R., Dickens, B.M, et Baudoin, J-L. (1995). La bioéthique, ses fondements et ses controverses. ERPI.

Tamariz, L., Palacio, A., Robert, M. et Marcus E.N., (2013). Improving the informed consent process for research subjects with low literacy: a systematic review. J Gen Intern Med, 28(1): 121-6. 
TITRE: LES PROJETS CLINIQUES À LA FRONTIÈRE DE LA RECHERCHE EXPÉRIMENTALE ET DU TRAITEMENT INNOVATEUR: QUEL CONSENTEMENT?

Auteur(s): Johane PATENAUde

PublicATION: RECRUTEMENT ET CONSENTEMENT À LA RECHERCHE : RÉALITÉS ET DÉFIS ÉTHIQUES

PAGES: $39-53$

ISBN: 978-2-7622-0357-8

Directeurs: Ana Marin, BÉATRICE Eysermann et Michel T. GIROUX

URI: HTTP://HDL.HANDLE.NET/11143/14106

DOI: HTTPS://DOI.ORG/10.17118/11143/14106 


\section{Les projets cliniques à la frontière de la recherche expérimentale et du traitement innovateur : Quel consentement?}

Johane Patenaude, professeure titulaire et chercheure, Département de chirurgie, Faculté de médecine et des sciences de la santé, Université de Sherbrooke

Résumé : Le consentement à un traitement expérimental (activité de recherche) exige la prise en compte d'informations plus exhaustives que celles exigées pour consentir à un traitement innovateur (activité clinique). D'où l'importance de déterminer adéquatement le statut auquel correspond effectivement le traitement proposé. Or les définitions visant à départager la recherche de l'innovation sont reconnues depuis longtemps pour être peu opérationnelles, entraînant des risques de confusion. Cette confusion possible a un impact direct sur le consentement, puisque la nature du consentement requis dépend du statut qu'on accorde à l'intervention concernée.

Ce problème est décrié depuis plus de 15 ans par de nombreux chercheurs, cliniciens, gestionnaires et autres parties prenantes. Où en sommes-nous aujourd'hui? Pratiquement au même point. C'est ce dont il sera question ici. À partir des principaux jalons de l'itinéraire de la notion de traitement innovateur depuis sa survenue dans les documents normatifs québécois, nous dégagerons quelques lieux de confusion quant à son opérationnalisation en recherche et en clinique. Finalement, quelques pistes de solution seront proposées.

Mots clés : Éthique, soins innovateurs, évaluation éthique de l’innovation, consentement.

Abstract: Consent to an experimental treatment calls for a more exhaustive consideration of the relevant information than that is required to agree to an innovative treatment (clinical activity). It is thus important to adequately determine the status to which the proposed treatment effectively corresponds. However, the definitions aimed at defining the difference between research and innovation have been recognized to be minimally operational for a long time, which is causing some risks of confusion. Such possible confusion has a direct impact on consent, since the very nature of the required consent depends on the status given to the intervention at stake.

This problem has been decried by numerous researchers, clinicians, managers and other stakeholders for more than 15 years. Where are we at today? Practically at the same point. This is what will be discussed here. Starting with the principal itinerary milestones of the notion of innovative treatment presented at the beginning of its occurrence in normative Quebec documents, we will extract statements where the confusion has been documented with respect to the operationalization aspect in research as well as in the clinical field. Finally, a few paths of solutions will be proposed.

Keywords: Ethics, innovative care, ethical evaluation of innovation, consent 


\section{Introduction}

Les années 90 donnent lieu à un foisonnement d'écrits et de communications en éthique de la recherche, notamment dans le cadre de la recherche biomédicale. La médecine occidentale contemporaine privilégiait déjà les connaissances fondées sur des données probantes (Sackett, 1997 : 3-5). Parallèlement, c'est sur cette base que les écrits de l'époque relancent l'importance de la recherche biomédicale scientifique en vue de nouvelles interventions diagnostiques et thérapeutiques au grand bénéfice des patients (Wilfond, 1995). Bien que jugées nécessaires, ces activités de recherche issues de données probantes soulevaient déjà des critiques au plan éthique en raison des risques encourus pour le patient et du besoin de rappeler que ces activités ne peuvent se faire aux dépens de valeurs éthiques fondamentales de notre société (Kerridge et coll., 1998; Tonelli, 1998), dont celles liées au respect de l'autonomie et qui se traduisent de manière privilégiée dans l'obtention d'un consentement libre et éclairé. Le défi posé par la société dans le continuum des activités de recherche, de l'innovation et de l'exercice de la médecine devenait celui d'établir les limites de l'acceptable (Desjardins, 1997).

Au Canada comme dans de nombreux pays, lorsqu'une nouvelle intervention médicale est utilisée sur une base expérimentale chez des sujets humains, on exige qu'un protocole applicable à ces sujets soit d'abord soumis à l'examen d'un comité d'éthique de la recherche (CER) pour approbation. Le contrôle des CER ne s'exerce plus à partir du moment où un traitement a dépassé l'étape expérimentale; ce dernier relèvera dès lors de la pratique clinique. Il existe toutefois une zone d'ombre entre le statut expérimental d'un traitement, considéré comme de la recherche et encadré comme tel par les CER, et le statut d'un traitement pleinement reconnu en clinique. Cette zone d'ombre, c'est le traitement ou soin dit «innovateur». Ce type de soins ne s'inscrit pas dans un contexte de recherche et vise plutôt l'intérêt d'un patient. Selon Avard et coll. (2004), se référant à Deleury (2002 : 145-147) : «lls consistent en une initiative spontanée d'offrir un traitement inédit, non validé, non éprouvé et non approuvé par les pairs». Par exemple, «[l]es thérapies nouvelles peuvent être des techniques

d'intervention chirurgicale, l'utilisation de nouveaux appareils d'investigation ou de traitement, des greffes d’organes, l'administration de médicaments, etc.» (Kouri et Philips-Nootens, 1996-97 : 108).

Ce statut intermédiaire, nous le verrons, soulève d'importants défis sur plusieurs plans, notamment au regard des informations requises à transmettre aux éventuels patients à qui ces traitements innovateurs seraient proposés dans le cadre de leurs soins, de leur compréhension de celles-ci et conséquemment au regard du respect du caractère libre et éclairé de leur consentement.

En effet, puisqu'un traitement qualifié d'innovateur n'est pas soumis à une évaluation éthique comme c'est le cas en recherche (celui-ci relevant plutôt de la pratique clinique) la nature et la portée du consentement du participant pourraient alors être très différentes selon la qualification donnée à la procédure, expérimentale ou innovatrice. Plusieurs projets impliquant de nouveaux soins font en sorte que la question se pose encore aujourd'hui de savoir s'ils relèvent de l'expérimentation sur l'humain ou s'ils s'inscrivent plutôt parmi les pratiques cliniques. De fait, la mince frontière qui sépare l'expérimentation et l'innovation, dans le continuum de la recherche et la clinique, peut occasionner des difficultés pour les CER, les cliniciens ou toute autre partie prenante ayant à déterminer si une procédure relève ou non de leur champ de compétences. 
Les professionnels de la santé, et particulièrement les médecins, peuvent également rencontrer des difficultés à déterminer dans quelle mesure leurs activités relèvent des soins cliniques ou de la recherche sur l'humain. En effet, la recherche clinique et l'exercice de la médecine ne s'effectuent généralement pas dans des milieux étanches; ils cohabitent souvent dans un même établissement de santé et par les mêmes intervenants qui cumulent les rôles de clinicien et de chercheur. Ce rapprochement entre les activités de recherche et de soins pourrait augmenter la confusion à l'égard de la finalité des procédures : faire avancer les connaissances ou soigner (Edgar and Rothman, 1995)?

L'un des effets néfastes de cette confusion serait de laisser libre cours à des traitements expérimentaux sur des patients en clinique sous le couvert de soins estimés innovateurs, voire approuvés. En conséquence, l'encadrement éthique et scientifique requis pour de telles activités de recherche clinique pourrait s'en trouver sensiblement réduit et ainsi exposer les patients à des risques indus ou auxquels ils n'auraient pas consenti librement ou en toute connaissance de cause. Cette préoccupation génère la question suivante : À quel moment les nouvelles interventions en clinique devraient-elles s'astreindre aux mêmes normes de protection des sujets que celles qui s'imposent aux chercheurs (Moutel et coll., 1998)? Répondre à cette question implique la capacité des décideurs de maîtriser les caractéristiques propres aux différents types d'activités qui jalonnent, tels un continuum, la recherche biomédicale et l'exercice de la médecine, ainsi que les dispositifs normatifs propres à chacun de ces types d'activités comme peut l'être, par exemple, le Comité d'éthique de la recherche dans le cas d'une activité de recherche expérimentale impliquant le concours de sujets humains. D'autres questions sollicitent ces compétences, comme celle de déterminer à quel moment la modification d'une technique standardisée représente-t-elle plus qu'une modification et devient-elle, du coup, un traitement expérimental, c'est-à-dire de la recherche (Patenaude et Cabanac, 2000).

Ces enjeux, loin d'être nouveaux (Moore, 1969; Blancquaert, 2000; Blancquaert et coll., 2001), sont plus que jamais d'actualité en raison de plusieurs facteurs, dont le délai de plus en plus court exigé entre le développement des connaissances issues de la recherche fondamentale et leurs possibles applications (sur le marché?) en clinique par les organismes subventionnaires, les promoteurs, voire les centres de recherche et les universités elles-mêmes. Cette rapidité hautement valorisée pourrait contribuer à intensifier la tentation néfaste que soulevaient déjà Kouri et Philips-Nootens en 1997 (103):

La tentation pourrait être grande, pour certains, de qualifier un acte de «soin innovateur» plutôt que de «thérapie expérimentale», car il serait alors possible pour les chercheurs de procéder sans contrainte externe. Il y aurait ainsi rétrécissement ou compression de la phase expérimentale permettant d'aboutir plus rapidement à la phase innovatrice, peut-être au détriment de la sécurité des personnes.

Ces auteurs poursuivent, soulignant à juste titre la teneur d'ordre éthique - plutôt que juridique - de ces enjeux:

Des situations factuelles très similaires peuvent ainsi mener à des qualifications différentes. Est-il vraiment opportun d'attendre le développement d'une «jurisprudence éthique» sur la question afin de pouvoir préciser l'acception exacte des expressions utilisées par le législateur? 
Si les soins innovateurs génèrent beaucoup de méfiance et d'inquiétude, particulièrement chez les bioéthiciens, juristes et cliniciens en Amérique du Nord (Kouri et Philips-Nootens, 1996-97; Agich, 2001; Blancquaert et coll., 2001; Patenaude et Cabanac, 2000; Kouri, 2013) et en France (Moutel et coll., 1998), ceux-ci n'en reconnaissent pas moins les avantages indéniables et la pertinence. Là n'est pas la question, on l'aura compris. Du reste, il convient aussi de noter que la situation inverse, c'est-à-dire de qualifier d'expérimental un traitement qui serait bien plutôt innovateur, serait tout autant susceptible d'engendrer des préjudices envers les patients à qui ces soins innovateurs sont destinés, ne serait-ce qu'en raison des délais indus impliqués pour l'obtention de l'approbation du CER et la préparation conséquente du dossier exigé à cette fin par le clinicien.

Agich (2001 : 296) résume bien cet enjeu. Selon lui, la demande d'un essai clinique dans un domaine de la médecine en cours de développement rapide et dynamique peut entraver l'innovation. Dans certaines circonstances, écrit-il, le retard dans le développement et l'adoption de tout nouveau traitement est un prix à payer. Chaque fois qu'il existe d'autres traitements standards avec un degré de succès acceptable et qui ne sont pas lourds, les essais cliniques sont justifiés. Cependant, poursuit-il, il est moins clair que les retards soient éthiquement justifiés dans les domaines de la médecine ou des maladies où il n'y a pas de traitements satisfaisants ou lorsque les traitements sont encombrants ou problématiques. Agich reconnaît néanmoins du même souffle que les critères aussi vagues que lourds ou problématiques sont, bien sûr, empreints de controverses et de matière à débat (2001 : 296).

Qu'en est-il de ces enjeux aujourd'hui, sommes-nous mieux outillés pour y faire face? Où en sommes-nous? Un tel bilan suppose de revenir sur quelques-uns des principaux concepts proposés dès le début des années 90 par les instances normatives québécoises et dont les définitions visaient justement à faciliter la détermination des procédures concernées dans un souci de respect de l'autonomie des personnes, notamment des personnes vulnérables, en assurant un plein consentement à l'intervention qui leur serait proposée dans le cadre de leurs soins ${ }^{1}$. Il n'est donc pas étonnant que le souci de définir ces concepts se soit manifesté par les instances normatives visant à encadrer les activités de recherche, dont l'expérimentation sur l'humain fait partie. Voyons-les brièvement.

\section{Quelques concepts et définitions}

Le Ministère de la Santé et des Services sociaux (MSSS) du Québec proposait, en 1998, les trois définitions suivantes dans son Plan d'action ministériel (PAM) en éthique de la recherche et en intégrité scientifique (MSSS, $1999: 25-26)$ :

Acceptée: Une procédure dont l'efficacité clinique, les indications et les protocoles sont bien établis sera désignée comme acceptée.

Il s'agit de soins standards pour lesquels le consentement aux soins est requis. Le CER n'est pas requis.

\footnotetext{
1. Bien qu'il s'agisse d'une revue des définitions dans le contexte québécois, il convient de noter que ces définitions, dans un contexte de recherche français, ont démontré la même confusion chez les décideurs, ainsi que les mêmes limitations conséquentes au regard des informations à transmettre aux patients participants à ces traitements à la frontière de l'expérimentation et du traitement innovateur (Moutel et coll.,1998; Patenaude et Cabanac, 2000). Nous ignorons malheureusement ce qu'il advient aujourd'hui de cette situation en France. Notre bilan se bornera ici à l'évolution de cette problématique au Québec, ainsi qu'ailleurs au Canada en raison du partage de plusieurs cadres normatifs en recherche en matière de consentement.
} 
Expérimentale : Par contre, une procédure dont l'efficacité clinique n’a pas encore été reconnue est désignée comme expérimentale. Nous ne savons pas si elle produit les bénéfices escomptés. Donc, on ne s'attend pas à ce qu'une telle procédure soit acceptée par les services de santé, sauf dans un protocole de recherche.

Le consentement à la recherche est requis par la signature d'un formulaire détaillé sur l'objet du projet, les implications à y prendre part, etc. Le CER assure ainsi l'évaluation éthique et le suivi du projet.

Innovatrice: Il reste des procédures qui ont dépassé l'étape expérimentale. Leur efficacité a été établie, mais, vu le manque d'expérience, les modalités d'application et même les indications exactes pour ces interventions sont à préciser. On appelle une telle technologie innovatrice [...].

Les procédures qualifiées d'innovatrices ne requièrent pas de consentement à partir d'informations détaillées comme dans le cas de la procédure expérimentale 2 . Cette définition de la notion de procédure innovatrice du PAM, mentionnée ci-dessus, provient du Conseil d'évaluation des technologies de la santé (CETS, 1991 : 2-3) qui la proposait en 1991 à l'occasion d'un rapport sur les transplantations d'organes au Québec. Selon le PAM, cette définition, qu'il juge limitée, aurait toutefois «permis d'atteindre, dans des domaines similaires, un consensus important tout en conservant la flexibilité nécessaire pour s'adapter au développement de la recherche» (MSSS, 1998 : 25). Or, rien n'est moins sûr, comme on le verra dans la partie qui suit.

\section{Procédure innovatrice ou expérimentale : quels repères, selon qui?}

Tenter de définir rigoureusement et de manière opérationnelle la notion de procédure innovatrice fut sans doute une épine de taille dans la chair du MSSS, notamment au regard de la rédaction de son PAM en éthique de la recherche et en intégrité scientifique. Ce passage du PAM (MSSS, 1998 : 25) en témoigne de lui-même:

Pendant la période de consultation de la commission parlementaire chargée d'examiner l'avant-projet de loi modifiant le Code civil en matière de recherche biomédicale, plusieurs organismes ont mentionné la difficulté qu'ils avaient d'arriver à une définition entièrement satisfaisante du concept de soins innovateurs. Ce constat est important puisque définir une activité comme s'il s’agissait de soins innovateurs plutôt que d'une activité de recherche soustrait par la suite celle-ci au mécanisme d'examen éthique, alors que c'est le comité d'éthique de la recherche qui détermine ce statut.

Dans le cas où il appartient au comité d'éthique de la recherche (CER) d'accorder un tel statut, il n'existe cependant pas d'exigences explicites quant à la nature et à la portée de l'information qui doit lui être soumise pour ce faire. On peut alors se questionner sur les critères mobilisés par les CER pour procéder à cette évaluation (la littérature n'en rend pas compte). De plus, on ignore si ces évaluations sont partagées d'une instance à l'autre ou si, au contraire, elles sont divergentes.

Ces questions furent à la base de l'une de nos études empiriques, qui a clairement démontré la confusion tant chez les 27 CER que chez les 37 cliniciens biochimistes à qui l'on demandait de qualifier le type de procédure concerné face à un même projet sur la base des trois définitions du MSSS énoncées précédemment (Patenaude et coll., 2008). Sans revenir sur les détails de cette enquête pancanadienne, il convient néanmoins d'en

\footnotetext{
2. Dans le cas d'activités jugées innovatrices, le PAM précise dans la définition quelques consignes sur leur portée générale ainsi que sur leur cadre global de réalisation: «Afin d’augmenter le niveau de connaissance, il est important de recueillir toute expérience future des applications de cette technologie de façon systématique et de communiquer ces expériences au monde médical. Pour cette raison ces activités ne doivent se poursuivre que dans une institution universitaire autorisée où les ressources requises sont disponibles » (MSSS, $1999: 26)$.
} 
reprendre ci-dessous les résultats obtenus pour trois de ses questions. Les résultats surlignés dans les tableaux qui suivent méritent attention.

Question

Selon-vous, est-ce que ce projet constitue de la recherche?

\begin{tabular}{|c|c|c|c|}
\hline & Biochimistes & CÉR & N \\
\hline Oui & 30 & 14 & 44 \\
\hline Non & 7 & 13 & 20 \\
\hline $\mathbf{N}$ & 37 & 27 & 64 \\
\hline
\end{tabular}

Question

Selon-vous, quelle procédure fait l'objet de ce projet?

\begin{tabular}{|l|c|c|c|}
\hline & Biochimistes & CÉR & N \\
\hline Procédure expérimentale & 3 & 2 & 5 \\
\hline Procédure innovatrice & 25 & 10 & 35 \\
\hline Procédure acceptée & 4 & 5 & 9 \\
\hline Autre & 5 & 9 & 14 \\
\hline $\mathbf{N} \backslash \mathbf{R}$ & 0 & 1 & 1 \\
\hline $\mathbf{N}$ & 37 & 27 & 64 \\
\hline
\end{tabular}

\begin{tabular}{|l|c|c|c|}
\hline \multicolumn{3}{|c|}{ Question } \\
\hline \multicolumn{3}{|c|}{ Selon vous, quelle procédure fait l'objet de ce projet? } \\
\hline CÉR & Biochimistes & CÉR & N \\
\hline CÉC (comité éthique clinique) & 16 & 12 & 28 \\
\hline Investigateurs & 8 & 2 & 10 \\
\hline DSP ou CMDP & 7 & 2 & 9 \\
\hline CÉR et CÉC & 0 & 8 & 8 \\
\hline Investigateurs et cliniciens impliqués & 3 & 0 & 3 \\
\hline Autres & 1 & 0 & 1 \\
\hline N/R & 1 & 3 & 4 \\
\hline N & 1 & 0 & 1 \\
\hline
\end{tabular}

Comme le décriait un groupe d'experts de l'Agence en évaluation des technologies et des modes d'intervention en santé du Québec (AETMIS-INESSS) : «The collection of validity and utility data is essential not only to further scientific knowledge but also to be able to adequately inform affected individuals» (Blancquaert et coll., 2001).

Il importe donc plus que jamais d'identifier les lieux de confusion possibles dans la compréhension, l'application et l'opérationnalisation des distinctions entre les types de procédures. De nombreux travaux théoriques issus des années 90 ont porté sur l'importance de mieux définir cette zone d'ombre incontournable qu'est le soin innovateur dans le continuum liant la recherche aux interventions approuvées en clinique (Prentice et coll., 1997; Fost, 1998; Lind, 1992; Edgar et Rothman, 1995; ACP-ASIM, 1998, et de nombreux autres). Un tel foisonnement convergeant dans la littérature n'est pas le fait du hasard; cette décennie fut le théâtre de l'apparition massive de plusieurs technologies dites «de rupture» en santé (génétique, génomique, clonage 
animal/humain, imagerie, neurosciences, nanotechnologies, implants de tout ordre, incorporation d'organismes génétiquement modifiés, néonatalogie, etc.). Un espace plus considérable que jamais émergeait alors entre le laboratoire et le cabinet du médecin. Cet espace, qui n’est ni recherche ni soin standardisé, restait donc à circonscrire.

Au Québec, le Conseil d'évaluation des technologies de la santé (CETS, 1991) situait l'innovation dans le chevauchement des procédures acceptées et celles dites expérimentales. Cette définition, nous l'avons vu, fut reprise telle quelle par le MSSS en 1998. Cette définition s'inscrivait dans la foulée de l'article 21 du Code civil du Québec (CCQ), qui, jusqu'en 2013, précisait : «Ne constituent pas des expérimentations les soins qui, selon le comité d'éthique, sont des soins innovateurs requis par l'état de santé de la personne qui y est soumise.» En apparence clair, cet article 21 énonçant certaines règles concernant l'expérimentation médicale demeurait une source de confusion tant pour les CER que pour les investigateurs et les cliniciens, car il vise à la fois la réglementation des soins expérimentaux, de l'expérimentation purement scientifique et des soins innovateurs, sans toutefois définir ni préciser la portée de ces termes (Kouri et Philips-Nootens, 1996-97; Weisstub et Verdun-Jones, 1997).

Procédure innovatrice, soin ou traitement innovateur... Ce travail de définition, bien que très complexe, visait alors à répondre à un besoin. Qu'en est-il de ce besoin aujourd'hui et de quels moyens disposons-nous collectivement pour y répondre?

\section{Où en sommes-nous?}

Ce travail de définition de procédures (acceptées, expérimentales ou innovatrice) visait, dès le début des années 90, à répondre au besoin d'encadrer les procédures innovatrices en les distinguant des autres pour assurer dans ce cas l'obtention d'un consentement conséquent de patients ou participants éventuels. Le PAM ciblait clairement ce besoin en 1998, en précisant (26) : «Afin de guider les comités d'éthique de la recherche et de les doter de balises communes s'agissant de la notion de soins innovateurs - le ministère n'y était pas encore parvenu efficacement - , le Ministère de la Santé et des Services sociaux du Québec confie au FRSQ³, au CQRS ${ }^{4}$, au Collège des médecins du Québec ainsi qu'au CETS le mandat de préciser davantage le concept de soins innovateurs dans le cadre de l'application de l'article 21 du Code civil». Un appel à tous fut ainsi lancé.

Un long silence s'ensuivit. On ne s'étonne donc pas que Sonia Audy, mandatée pour revoir l'ensemble de la politique ministérielle (PAM), n'ait pu consacrer plus qu'une quinzaine de lignes dans son rapport sur cette question. On ne s'étonne pas non plus du titre choisi pour cette section du rapport, «Les soins innovateurs : un dossier dont personne ne veut...» (Audy, 2006 : 253).

Il faudra attendre les années 2008-2009, soit 10 ans, pour y trouver écho. C'est le FRSQ (aujourd'hui le FRQS) qui donna suite à cet appel dans son document intitulé Les standards du FRSQ sur l'éthique de la recherche en santé humaine et l'intégrité scientifique. Cet important document y consacrait une section d'une demi-page intitulée «L'expérimentation et les soins innovateurs» (FRSQ, 2008, Section 25 : 41), essentiellement calquée sur le CCQ:

3. FRSQ : Fonds de recherche en santé du Québec, devenu aujourd'hui le Fonds de recherche du Québec en santé (FRQ-S).

4. CQRS : Conseil québécois pour la recherche sociale 
L'article 21, al. 4 du Code civil du Québec traite les expérimentations et les soins innovateurs comme étant des interventions distinctes: «Ne constituent pas des expérimentations les soins qui, selon le comité d'éthique, sont des soins innovateurs requis par la personne qui y est soumise.»

L'importance de cette distinction tient au fait que les soins innovateurs peuvent être dispensés suivant les normes juridiques applicables aux soins requis par l'état de santé plutôt que d'après les normes qui concernent l'expérimentation.

Le Code civil ne définit pas ce que sont les soins innovateurs et l'expérimentation. Pour le moment, il revient au CER d'identifier ce qui caractérise chacun de ces deux types d'interventions lorsque cette distinction doit être faite.

Le FRSQ fut sans doute conscient du fait que son propos n'ajoutait pas d'eau au moulin, car il termine ainsi : "Le FRSQ produira un document qui interprète et clarifie les trois concepts suivants, que contient l'article 21 du Code civil : "projet de recherche", "expérimentation" et "soins innovateurs"».

Ce projet n'a pas eu de suite; il semble que le FRSQ (ou FRQS) ne produira pas ce document interprétant et clarifiant les concepts qui figuraient au quatrième et dernier alinéa du CCQ. En effet, parmi les modifications apportées au Code civil du Québec en juin 2013, on remarque que cet alinéa, qui portait sur les soins innovateurs, a été supprimé. Conséquemment, il ne revient plus aux CER de déterminer le statut, expérimental ou innovateur, d'une procédure. Les procédures innovatrices relevant juridiquement des soins dans le CCQ. depuis 2013, il revient donc désormais au clinicien, et non au CER, de déterminer ce statut. Si cette nouvelle posture du CCQ compense certaines faiblesses au plan juridique ${ }^{5}$, la zone d'ombre demeure entière au plan éthique. Comme le soulève Viens (2015: 16):

Tel qu'établi jusqu'en 2013 par le libellé de l'article 21 C.c.Q., le CER possédait une grande responsabilité quant à la détermination de la nature d'un acte thérapeutique donné. Lorsque ce dernier était considéré comme un soin innovateur requis par l'état de santé de l'individu, il était incertain si les règles relatives aux soins courants ou celles applicables à la recherche s'appliquaient. Par opposition, lorsque le comité d'éthique concluait à la recherche à visée thérapeutique, la protection de l'article 21 C.c.Q. était assurée. Maintenant que l'exception relative aux soins innovateurs n'est plus au Code civil, l'incertitude demeure tout de même quant aux règles applicables à ces derniers. Les soins innovateurs sont-ils assujettis aux règles relatives aux soins courants ou à la recherche?

Le problème, on le voit, reste entier au plan éthique : quel statut, donc quel consentement?

\section{Pistes de solution}

La littérature médicale internationale abonde de pistes de solution, au moins théoriquement, particulièrement dans les spécialités chirurgicales (Broekman et coll., 2016). Malheureusement, il existe peu ou pas de littérature ou de rapport rendant compte de leur mise en œuvre effective dans les milieux de pratique. La situation ne semble pas plus simple du côté de la gouvernance, comme en témoigne l'initiative d'un récent projet de loi au Royaume-Uni visant à encadrer les cliniciens dans la détermination du statut, innovateur ou non, accordé à une procédure innovatrice envisagée pour leurs patients. Selon Kouri (2013, note p. 875) :

5. Kouri (2013: 875$)$ précise : «Plus fondamentalement cependant, cet alinéa comportait une faiblesse évidente : comment un comité d'éthique pourrait-il déterminer d'avance si des soins sont innovateurs ou non ? Logiquement, une telle détermination en serait une de nature strictement scientifique. Qu'il s'agisse d'innovation ou d'expérimentation, dans un cas comme dans l'autre, l'on se retrouve dans le domaine de la recherche et l'on doit, de toute façon, se conformer aux exigences du Code civil ». 
[...] c'est en partie pour cette raison que le projet de loi intitulé «Medical Innovation Bill (N²), 2013-14 (Bill 107)», fut présenté en première lecture à la Chambre des Communes du Royaume (Parliament UK, 2013). On y proposait que tout soin innovateur doit être approuvé par une équipe multidisciplinaire de médecins («multidisciplinary team of doctors»).

Ce projet de loi a cependant été retiré et ne progressera plus, et le site internet dédié n'en donne malheureusement pas la raison. Une telle piste d'évaluation et de décision en comité de pairs semble néanmoins prometteuse si, bien sûr, les membres disposent d'un cadre de référence et de critères clairs et partagés pour guider leur jugement dans l'exercice de ce que les philosophes éthiciens nomment la pensée pratique. À défaut, nous pourrions voir se répéter des résultats semblables à ceux de notre enquête pancanadienne et dont nous faisions sommairement état plus tôt.

Des propositions de solutions furent aussi présentées dans le cadre de la psychopharmacologie. Le titre du principal article dans le domaine parle de lui-même : «The ethics of clinical innovation in psychopharmacology: Challenging traditional bioethics» (Ghaemi et Goodwin, 2007). À l'instar de plusieurs cliniciens, dont Agich (2001), les auteurs insistent sur le manque de reconnaissance des bioéthiciens à l'égard de la spécificité de cette zone d’ombre que sont les traitements innovateurs. Néanmoins conscients des enjeux éthiques liés à cette phase intermédiaire, ces auteurs déplorent les limites de la bioéthique traditionnelle - et, du coup, celle des bioéthiciens qui s’y réfèrent - pour répondre aux enjeux éthiques qui y sont soulevés. Selon Ghaemi et Goodwin (2007) (c'est nous qui traduisons) :

Une évaluation conceptuelle et historique de ce sujet souligne l'importance éthique et scientifique de l'innovation clinique. Éthiquement, cela touche une limite qui, à notre avis, n'est pas adéquatement encadrée dans la bioéthique traditionnelle contemporaine. À l'heure actuelle, la recherche est considérée comme ne bénéficiant pas du tout aux patients qui y participent, tandis que les soins cliniques sont considérés comme étant uniquement pour le bénéfice des patients. L'innovation clinique chevauche ces deux mondes, parfois malaisément. Bien que plusieurs soutiennent que l'innovation clinique doit être évitée ou s'inscrire dans des projets de recherche, nous soutenons que l'innovation clinique est nécessaire pour progresser dans la recherche en psychopharmacologie et qu'elle pourrait mieux prospérer mieux si elle était guidée par les principes éthiques suivants : 1) le traitement devrait porter sur la base d'une hypothèse viable; 2) dans la mesure du possible, les observations cliniques devraient être signalées afin qu'elles puissent être évaluées par la communauté scientifique; 3) on devrait être disposés à signaler des observations inattendues des effets sur les médicaments; 4) un niveau élevé de consentement éclairé devrait être maintenu.

Ghaemi et Goodwin (2007) insistent pour dire que cette proposition va à l'encontre de la vision qui prévaut chez les bioéthiciens et selon laquelle la recherche et les soins cliniques sont des activités catégoriquement opposées, comme l'explique l'un ou l'autre ou la dichotomie du rapport Belmont sur la bioéthique. En effet, selon ces auteurs, cette approche a tellement polarisé leur profession en tant que cliniciens par rapport aux chercheurs, que de nombreux cliniciens n'appliqueront pas les nouvelles connaissances produites par la recherche clinique jusqu'à ce qu'elle soit incorporée dans les lignes directrices formelles de traitement, alors que, d'autre part, les chercheurs ont peu d'éléments pour les guider au regard du type de nouvelles connaissances les plus importantes à fournir.

Cette proposition «hybride» de Ghaemi et Goodwin (2007), empruntant tant à la recherche qu'à la clinique, trouve un certain écho chez plusieurs auteurs, dont Blancquaert et coll. (2001 : 106-107). Pour ces derniers, une fois que le statut des soins innovants est accordé, le manque de suivi scientifique et éthique qui en résulte 
pourrait avoir de graves conséquences. Compte tenu des incertitudes entourant les soins innovants, le suivi des patients se soumettant à ces traitements devient essentiel. Cette période de suivi doit, selon eux, être utilisée pour en mesurer l'impact et pour mettre à jour les données requises pour un consentement éclairé. En effet, selon Blancquaert et coll. (2001), seule une boucle d'information récursive peut assurer un véritable consentement éclairé aux essais suite à la phase de transition. La proposition de Blancquaert et coll. (2001) semble cependant plus engageante au plan éthique que celle de Ghaemi et Goodwin (2007), en soutenant que le concept de soins innovants, par opposition à l'expérimentation, ne devrait être invoqué que si un suivi scientifique et éthique est assuré.

Si ces pistes de solutions sont intéressantes pour plusieurs, d'autres les jugent contre-productives, dont Agich (2001 : 296), qui estime que le problème est plus profond et qu'il ne saurait être résolu par de telles pistes hybrides. Selon lui, dans le soin innovant, on a recours à des protocoles cliniques traitant des préoccupations cliniques pratiques et des paramètres finaux plutôt qu'à des hypothèses ou des méthodes scientifiques. SeIon lui, ces protocoles articulent les objectifs cliniques et intègrent les processus complexes de découverte et d'expérience acquise avec une procédure de développement. Ils peuvent, dit-il, être remarquablement différents des protocoles scientifiques qui découlent d'une déclaration claire d'une question de recherche sous les contraintes relativement rigides de la méthodologie scientifique. L'écart entre les postures précédentes et celle d'Agich (2001) semble se creuser davantage quand il insiste à l'effet que l'innovation tolère, et prospère même, sur la base de l'intuition, de l'expérience et de l'incertitude, alors que la recherche scientifique se déroule sous les contraintes méthodologiques d'une base de connaissance établie vers son but de confirmation ou de rejet d'hypothèses. Ainsi, il semblerait que l'écart entre recherche clinique et innovation clinique qu'indique Agich (2001) soit d'ordre épistémologique.

Pour notre part, il semble clair qu'une solution à ces enjeux ne peut être que le fruit d'une collaboration forte et soutenue entre les diverses parties prenantes, par exemple au sein d'un comité d'éthique clinique de l'innovation (CECI) qui serait reconnu par les instances des établissements de santé et les institutions universitaires et qui serait composé d'acteurs issus de la recherche (centre de recherche hospitalier ainsi qu'universitaire, le cas échéant), de la pratique clinique, de la gestion des soins, d'associations des usagers et de toute autre partie prenante. La participation active à ce comité de l'Unité d'évaluation des technologies et des modes d'intervention en santé et services sociaux (UETMISS) de ces établissements ${ }^{6}$ serait également souhaitable. La seule mise en place d'une telle instance ne serait évidemment pas une garantie de succès; les compétences et l'expérience de chacun devront être mobilisées dans une entreprise de coapprentissages et de valeurs partagées au service d'une innovation responsable dans nos institutions.

La tâche est plus que jamais complexe dans le contexte où les collaborations de recherche université-industrie ont été essentielles à un haut degré d’innovation technologique en médecine. Récemment, cependant, des questions critiques ont été posées sur les aspects négatifs potentiels des moyens hautement productifs d'encourager l'innovation. Les préoccupations portent sur les rôles flous entre la recherche académique et le monde commercial et les implications de la volonté nouvelle des universités de bénéficier financièrement de leur propriété intellectuelle. Comme le soulèvent Gelijns et Thier (2002), les rôles des deux parties sont souvent insuffisamment pris en compte en considérant que les membres des facultés universitaires sont consacrés à l'avancement des connaissances fondamentales et des entreprises industrielles en tant que simples déve-

6. L'Unité d'évaluation des technologies et des modes d'intervention en santé et services sociaux (UÉTMISSS) aide les décideurs des établissements de santé en réalisant, en soutenant et en encadrant des projets d'évaluation de technologies et de façons de faire en santé et services sociaux. L'UETMISS évalue ou encadre tout projet d'évaluation qui touche les équipements de haute technologie, la dispensation et l'organisation des soins, les nouvelles pratiques, les interventions médicales et chirurgicales, les équipements, les systèmes de soutien, les diagnostics, les médicaments, etc. 
loppeurs de recherche universitaire. Au contraire, l'innovation médicale dépend d'interactions approfondies entre les universités et l'industrie, les connaissances et le transfert de technologie dans les deux sens. Ces interactions ont eu d'importants avantages pour la santé publique et l'économie.

Pourtant, il existe un risque pour la relation université-industrie si les principes culturels et éthiques d'un partenaire maîtrisent ceux de l'autre. Par conséquent, les universités, les centres de recherche hospitaliers et l'industrie doivent maximiser les retombées de la collaboration par un encadrement socialement responsable et minimiser les inconvénients au moyen d'un changement organisationnel interne.

Oeuvrant, chemin faisant, au cœur de ces enjeux complexes, un comité d'éthique clinique de l'innovation (CECI), multidisciplinaire et multicomposite par essence, ferait œuvre de bioéthique contemporaine; chacun devenant du coup bioéthicien de fait. Ce rôle est plus complexe que jamais et, plus que jamais, il ne peut pas être assumé que par un seul individu, aussi expert soit-il. Il est temps de relever le défi qu'indiquait Agich :

It is time for bioethicists to think innovatively about the ethical problems associated with innovation in patient care. To do so, bioethics will need to pay closer attention to the actual clinical, institutional, and professional processes that operate in the development of novel therapeutic interventions (Agich, 2001: 296).

Plus concrètement, une telle instance d'évaluateurs avisés, comme le serait un CECl par exemple, pourrait relever le défi que soulevait Blancquaert et ses collègues (2001: 107) à l'effet que «les décideurs devront se préparer au défi du transfert de technologie [...] et travailler à la construction d'un cadre pour superviser la mise en œuvre des tests et établir des modalités pour leur intégration dans les services cliniques». À cette fin, ajoutent-ils, les méthodes qui favorisent la collecte de données sur la validité analytique et clinique des tests et sur leurs avantages et risques associés devraient faire l'objet d'une évaluation indépendante et multidisciplinaire des données pertinentes, ces évaluations devant être intégrées au processus décisionnel.

Qu'on ne s'y trompe pas, ce défi d'évaluation de nouvelles procédures et de leurs conditions d'intégration dans le processus décisionnel que soulèvent Blancquaert et ses coll. (2001) vise un transfert des connaissances qui soit éthiquement responsable (Legault et coll., 2017).

\section{Conclusion et perspectives}

S'il est de bon ton de dire que l'éthique est une responsabilité partagée, il convient néanmoins d'en assumer les conséquences en outillant efficacement les parties prenantes de nos institutions de recherche et établissements de santé, dont les CER, en vue d'une évaluation éthique plus assurée de ces initiatives menées avec et auprès de populations souvent vulnérables. Ce souci éthique dans le développement et l'adoption de nouvelles pratiques en santé contribuerait à réduire l'un des effets néfastes de cette confusion sur le respect de l'autonomie des personnes et leur droit à consentir. Ces valeurs sociales seraient fragilisées du moment où il serait relativement facile de laisser libre cours à des traitements expérimentaux sur des patients en clinique sous le couvert de soins jugés innovateurs, voire approuvés, selon l'évaluation subjective de chacun. Cette solution, de juger du caractère «recherche» ou «innovateur» selon la seule intention du clinicien, à savoir s'il entend acquérir de nouvelles connaissances ou soigner, bien qu'intéressante, est nettement insuffisante, voire naïve, dans nos contextes de pratique qui valorisent le cumul des rôles professionnels ainsi que la rapidité des applications en clinique des connaissances issues de l'expérimentation humaine. 
Les conflits de rôles, loin d'être rares, sont souvent structurels (Ghaemi and Goodwin, 2007). Des pistes sont donc à explorer dans la foulée de celles proposées dans cet article. D'ici là, la qualité de l'encadrement éthique de certains projets visant de nouvelles pratiques cliniques pourrait s'en trouver sensiblement réduit et les patients pourraient ainsi être exposés à des risques auxquels ils n'auraient pas consenti librement et en toute connaissance de cause.

Plus encore, la notion de traitements, procédures ou soins innovateurs est plus orpheline que jamais, voire abandonnée, depuis la suppression du dernier alinéa de l'ancien article 21 du CCQ en juin 2013 la concernant spécifiquement. En effet, bien que ce retrait règle certains problèmes au plan juridique, il n'en va pas de même au plan éthique. En effet, sur ce dernier plan, le problème reste entier. Le statut innovateur d'une procédure, soin ou traitement, existe bel et bien; car il est une partie intégrante du continuum traversant le développement des connaissances au service des soins, de la recherche à la clinique, et il ne saurait en être autrement. La question de l'encadrement éthique de cette zone d'ombre subsiste néanmoins. Le but ultime de cet article est donc de sensibiliser les communautés concernées, en recherche comme en clinique, à l'importance, voire l'urgence, de répondre aux enjeux éthiques grandissants auxquels la notion de procédures ou soins innovateurs visait précisément à répondre et aux enjeux éthiques en découlant directement, dont ceux liés au respect de l'autonomie de la personne par l'obtention d'un consentement éthiquement valide ainsi que ceux liés à un transfert des connaissances socialement responsable.

\section{Remerciements}

Nous remercions chaleureusement les Instituts de recherche en santé du Canada (IRSC \# 142187) pour leur support à ces travaux, ainsi que Hubert Gagnon et Louise Corbeil pour leurs judicieux conseils. 


\section{Bibliographie}

American College of Physicians. (1998). ACP Ethics Manual (Fourth Edition). ISBN 0-943126-67.

Agich, GJ. (2001). Ethics and innovation in medicine, Journal of Medical Ethics, 27, 295-296

Audy, S. (2006). Le Plan d'action ministériel en éthique de la recherche et en intégrité scientifique : une entreprise insensée? Ministère de la Santé et des Services sociaux. Québec. Sect. 6.2.2. : 253.

Avard, D., Knoppers, M.B. et Lévesque, E. (2004). La génétique et le cadre juridique applicable au secteur de la santé; examens génétiques, recherche en génétique et soins innovateurs, 64 R. du B. 57.

Blancquaert, I. (2000). Availability of Genetic Services: Implementation and Policy Issues, Community Genetics, 3 (4), 179-183.

Blancquaert, I., Cleret de Langavant, G., Bouchard, L., Obadia, A., Chikhaoui, Y. and Battista, R.N. (2001). Oversight Mechanisms for Technology. Transfer in Molecular Genetics. Meeting the Challenge, Isuma, 103-108.

Broekman, M.L., Carrière, M.E. et Bredenoord, A.L. (2016). Surgical innovation: the ethical agenda. A systematic review. Medicine (Baltimore), Vijayaprasad Gopichandran (ed), 95 (25), 1-5.

Conseil d'évaluation des technologies de la santé (CETS). (1991). La transplantation au Québec. Rapport préliminaire sur l'efficacité, les coûts et les caractéristiques organisationnelles. Montréal.

Deleury, E. (2002). Le droit des personnes physiques, Cowansville, Éditions Yvon Blais.

Desjardins, L. (1997). Le consentement à l'expérimentation clinique : Quelles sont les limites de l'acceptable et de l'inacceptable? Journal du Barreau, 29 (4). Repéré à http://www.barreau.qc.ca/pdf/journal/vol29/ no4/consentement.html

Edgar, H. et Rothman, D.J. (1995). The institutional review board and beyond: future challenges to the ethics of human experimentation. Milbank Q., 73 (4), 489-506.

Fonds de la recherche en santé du Québec (FRSQ). (2008; Modifié par le conseil d'administration du FRSQ en décembre 2009). Les Standards du FRSQ sur l'éthique de la recherche en santé humaine et l'intégrité' scientifique. 51 pages.

Fost, N. (1998 Jun). Ethical dilemmas in medical innovation and research: distinguishing experimentation from practice, Semin Perinatol. 22 (3), 223-232.

Gelijns, AC. and Thier, SO. (2002). Medical Innovation and Institutional Interdependence Rethinking University-Industry Connections, Journal of the American Medical Association (JAMA), 287 (1), 72-77.

Ghaemi, S.N. et Goodwin, F.K. (2007). The ethics of clinical innovation in psychopharmacology: Challenging traditional bioethics», Philosophy, Ethics, and Humanities in Medicine, 2 (26) (Open access).

Kerridge, I., Lowe, M. and Henry, D. (1998 Apr). Ethics and evidence based medicine. BMJ., 11 (316[7138]), 1151-1153.

Kouri, R.P. (2013). Observations concernant certains changements apportés au code civil par la loi modifiant le code civil et d'autres dispositions législatives en matière de recherche. Revue de Droit Université de Sherbrooke (RDUS), 43, 867-886.

Kouri, R.P. et Philips-Nootens, S. (1996-97). L' «expérimentation» et les «soins innovateurs» : l'article 21 CCQ. et les affres de l'imprécision. Revue de droit de l'Université de Sherbrooke (R.D.U.S), 27, 89-137, 96. 
Legault, G-A., Verchère, C. and Patenaude, J. (2017 Apr.). Support for the Development of Technological Innovations: Promoting Responsible Social Uses, Science and Engineering Ethics, 1-21. DOI 10.1007/s11948017-9911-5. (Open Access).

Lind, S.E. (1992). Innovative medical therapies: between practice and research. Clinical Research. 36, 546-551.

Moore, F.D. (1969). Therapeutic Innovation: Ethical Boundaries in the Initial Clinical Trials of New Drugs and Surgical Procedures, Daedalus, The MIT Press on behalf of American Academy of Arts \& Sciences, 98 (2), 502522.

Moutel, G., Leroux, N. and Hervé, C. (1998 Apr.). Analysis of a survey of 36 French research committees on intracytoplasmic sperm injection. The Lancet, 351, 1121-1123.

MSSS. Ministère de la santé et des services sociaux. Direction générale de la planification et de l'évaluation. Gouvernement du Québec, (1998, Juin [Dernière modification : 14 avril 1999]).Plan d'action ministériel en éthique de la recherche et en intégrité scientifique. 40 pages.

Parliament UK., 2013. Medical Innovation (No.2) Bill 2013-14. Repéré à http://services. parliament.uk/ bills/2013-14/medicalinnovationno2.html

Patenaude, J. et Cabanac, J. (2000). Quelle recherche, quelle évaluation? Le cas de la distinction entre soin expérimental et soin innovateur. Éthique Publique, 2, 89-98.

Patenaude, J., Grant, A., Xhignesse, M., Leblanc, F. et Courteau, J., (2008 Jan). Evaluation of Clinical Innovation: A Gray Zone in the Ethics of Modern Clinical Practice? Journal of General Internal Medicine (JGIM), 23 (Suppl 1), 27-31.

Prentice, E.D., Gordon B.G. and Lin M.H. (1997). Determining when a clinical activity should be classified as research requiring institutional review board review. The Journal of Extra-Corporeal Technology, 29 (2), 88-91.

Sackett, D.L. (1997). Evidence-based medicine. Dans Zipursky, A. (dir.). Seminars in Perinatology. Fatal and Neonatal Hematology for the 21st Century. 21 (1), 3-5.

Tonelli, M.R., (Dec1998). The philosophical limits of evidence-based medicine. Academic Medicine, 73 (12), 1234-1240.

Viens, E-J. (2015). Le consentement des personnes vulnérables à la recherche: regard sur les amendements et les omissions de l'article 21 du code civil du Québec. Mémoire de maîtrise en droit. Faculté de droit. Université de Sherbrooke.

Wheeler, R. 2000, 3 June. «One person's innovation is another's experiment». BMJ. 320 : 1548.

Weisstub, D.N. et Verdun-Jones, S. (1996-97). «Pour une distinction entre l'expérimentation thérapeutique et l'expérimentation non thérapeutique». Revue de Droit Université de Sherbrooke (R.D.U.S.), 27, 49-87.

Wilfond, B.S. (1995 May-June). Screening policy for cystic fibrosis: the role of evidence. Hastings Center Report, S21-3. 
TITRE: L'INCERTITUDE AU CEUR DE LA TENSION ÉTHIQUE DU RECRUTEMENT EFFECTUÉ PAR LE CLINICIEN-CHERCHEUR

Auteur(s): Bruno LeClerC et AleXandra DubÉ-Loubert

PublicATION: RECRUTEMENT ET CONSENTEMENT À LA RECHERCHE : RÉALITÉS ET DÉFIS ÉTHIQUES

PAGES: $54-64$

ISBN: 978-2-7622-0357-8

Directeurs: Ana Marin, BÉatrice Eysermann et Michel T. Giroux

URI: HTTP://HDL.HANDLE.NET/11143/14108

DOI: HTTPS://DOI.ORG/10.17118/11143/14108 


\section{L'incertitude au cœur de la tension éthique du recrutement effectué par le clinicien-chercheur}

Bruno Leclerc, éthicien et président suppléant, CER, CISSS du Bas-Saint-Laurent Alexandra Dubé-Loubert, coordonnatrice, CER (2012 - 2016), CISSS du Bas-Saint-Laurent

Résumé: Pour assurer que le consentement aux essais cliniques soit donné volontairement quand le chercheur est le médecin traitant du patient, plusieurs CER conseillent de confier le recrutement à un tiers professionnel. Sachant que l'une des motivations de plusieurs cliniciens-chercheurs, notamment en oncologie, est de donner accès à leurs patients à des traitements expérimentaux prometteurs, ce conseil est-il toujours pertinent? En proposant une lecture critique des concepts de méprise thérapeutique et d'équilibre clinique, notre réflexion met en relief l'incertitude inhérente aux traitements dits standards aussi bien qu'aux essais cliniques, pour soutenir que le clinicien-chercheur est, sauf exception, le mieux placé pour procéder au recrutement. Le rôle du CER est alors d'entrer en dialogue avec le chercheur pour cerner les enjeux éthiques spécifiques au protocole et le guider en conséquence dans sa démarche de sollicitation des patients.

Mots-clés : recrutement, consentement, équilibre clinique, méprise thérapeutique, dialogue. 


\section{Introduction}

Notre présentation situe la problématique du recrutement dans le champ des essais cliniques de pharmacologie. Le recrutement s'y définit comme le premier contact entre un membre de l'équipe de recherche et le patient au cours duquel le patient est informé de l'existence de l'essai thérapeutique et de la possibilité d'y participer. II s'agit donc de recrutement direct. Nous n'abordons pas ici les enjeux du recrutement indirect par la diffusion d'affiches, d'encarts publicitaires ou, de manière plus personnalisée, par la transmission d'une lettre de sollicitation.

Selon l'Énoncé de politique des trois Conseils : Éthique de la recherche avec des êtres humains (EPTC 2), qui traite principalement du recrutement à l'article $3.1 \mathrm{~A}$, «le consentement doit être donné volontairement» (EPTC 2, 2014:28) et il doit être conçu comme le processus continu d'adhésion à une proposition de recherche qui débute avec le premier contact. Ce dernier peut être l'occasion d'initier involontairement une influence indue réduisant le caractère libre et volontaire du consentement. Cette qualité essentielle du consentement «s'accorde avec le respect de la dignité humaine et signifie que la personne a choisi de participer à la recherche en fonction de ses valeurs, de ses préférences et de ses désirs» (EPTC 2, 2014 : 28).

Notre question de recherche est la suivante : Les motivations et les valeurs du clinicien-chercheur, qui se situent en amont du recrutement, peuvent-elles introduire un biais mettant en cause le caractère libre du consentement, et ce, dès le premier contact? Selon certaines études, en effet, la moitié des oncologues chercheurs auraient pour motivation de donner accès à une molécule prometteuse, par exemple une molécule non homologuée au Canada (Thong et coll., 2016).

Dans ce corridor de pratique précis naissent des questions corollaires de recherche : Comment baliser l'espoir d'un bénéfice thérapeutique qui entre en tension avec l'acceptation du risque expérimental? En vue de bien cerner cet enjeu éthique, le comité d'éthique de la recherche (CER) doit-il chercher à connaître les raisons d'agir, les motivations et les valeurs des cliniciens-chercheurs? Est-il même légitime pour un CER de partager certaines raisons d'agir du clinicien-chercheur? Nous y reviendrons.

Cernons d'abord le cœur du questionnement éthique à l'intersection de deux concepts souvent mobilisés dans la littérature pour examiner la tension entre motivation thérapeutique et risque expérimental, ceux de méprise thérapeutique et d'équilibre clinique (clinical equipoise). Le premier contact motivé par l'intention thérapeutique peut-il induire une méprise thérapeutique? Le concept d'équilibre clinique peut-il agir comme régulateur entre les impératifs de la recherche et ceux de la clinique?

Plaçons les définitions des concepts clés:

- Équilibre clinique : incertitude relative entre le traitement standard et le traitement expérimental, pour une condition donnée, qui justifie la tenue d'une étude clinique (Fried, 1974; Friedman, 1987; Webster et coll., 2016).

- Méprise thérapeutique : confusion, chez le participant d'une étude clinique, entre les impératifs de la recherche et ceux de la pratique clinique (Applebaum et coll. 2012).

- Impératifs de la recherche : mise en place des mesures et des méthodes nécessaires à une investigation systématique visant à établir des faits, des principes ou des connaissances généralisables. (EPTC, 1998, 1.1) 
- Impératifs de la clinique : prodiguer les soins nécessaires à la guérison ou à l'amélioration de la santé ou de la qualité de vie en fonction des données probantes. ${ }^{1}$

Nous avons retenu la définition classique de la méprise thérapeutique proposée par Applebaum et coll. (2012). Elle indique bien la tension conceptuelle en cause, et ce, bien qu'elle soit formulée en des termes abstraits qui rejoignent assez peu le vécu du patient, qui ne pense pas en termes d'impératifs et de pratiques cliniques ou de recherche, mais en termes de bénéfices, d'espoir, de soulagement ou de guérison.

Nous reviendrons plus loin à cette importante dimension motivationnelle, et, en cours d'argumentation, une autre distinction significative sera faite, soit entre les études thérapeutiques et non thérapeutiques. Mais l'analyse qui débute maintenant porte sur les études thérapeutiques.

Nous avançons que les concepts d'équilibre clinique et de méprise thérapeutique peuvent être source de confusion dans l'évaluation éthique des essais cliniques. À propos d'équilibre clinique, on peut en effet soutenir que l'incertitude réelle quant aux thérapies les plus efficaces justifie la tenue d'études. Quant à la thérapie expérimentale, est-elle toujours susceptible d'interférer avec les objectifs de soins?

\section{Clinical equipoise / équilibre clinique}

Tel que mentionné précédemment, nous définissons l'équilibre clinique comme l'incertitude relative entre le traitement standard et le traitement expérimental pour une condition donnée. Cette incertitude justifierait la tenue d'études pour établir l'efficacité relative entre un traitement standard et un traitement expérimental. Deux questions se posent au sujet de l'équilibre clinique : Quelle règle d'évaluation ou quelles lignes directrices peut-on dériver du débat autour de ce concept? Quelle serait l'utilité d'une telle règle pour évaluer les enjeux éthiques du premier contact, du recrutement à la recherche?

Nous ne suivrons pas tous les méandres du débat évolutif autour de ce concept. Nous prendrons appui sur l'étude-terrain de Webster et coll. (2016) pour jeter un regard rétrospectif sur quelques difficultés qui sont soulevées par ce concept et débattues dans la littérature en vue d'esquisser quelques pistes d'examen éthique liées à la reconnaissance de l'incertitude.

Webster et coll. montrent que l'évaluation éthique d'une étude clinique est largement déterminée par des jugements de valeur dans l'interprétation des données probantes, notamment par une forte croyance dans les standards de pratique. Il en conclut que l'equipoise est une question de croyances et que ce principe pourrait bien ne pas pouvoir constituer un obstacle solide à la tenue d'études futiles, donc qu'il pourrait bien ne pas être aussi utile qu'espéré.

Notre expérience au CER nous amène à préciser l'analyse de Webster et coll. (2016) : l'expérience avec un traitement - qu'il soit standard ou expérimental - est susceptible de peser sur les préférences cliniques d'une équipe. À ce propos, nous croyons qu'il existe une grande variabilité dans les préférences cliniques des médecins-chercheurs, même dans ce qu'on identifie comme standards de soin, chaque option présentant un équilibre particulier entre bénéfices, coûts et incertitudes; cet équilibre est au moins en partie dépendant du contexte de soins. Qui plus est, des approches cliniques dites standards peuvent être controversées ou faire l'objet de désaccords. On parle même parfois de culture clinique variable d'une institution à l'autre. Par

1. Nous avons formulé cette définition pour cadrer la discussion en rapport à notre connaissance ou perception des pratiques cliniques. 
exemple, certaines équipes se sentiront à l'aise avec une molécule expérimentale pour une nouvelle indication parce qu'ils ont une expérience positive de cette molécule : connaissance de ses effets secondaires les plus courants, capacité d'intervention rapide et efficace, etc.

Ce qui nous ramène aux jugements d'expérience et de valeurs en cause dans la façon dont les options thérapeutiques seront présentées aux patients, y compris les options expérimentales. Devons-nous adhérer pour autant à la conclusion de Webster et coll. (2016) sur la faible utilité du critère d'equipoise? Le facteur principal que nous retenons pour traiter cette question est la subjectivité inhérente à l'évaluation des options thérapeutiques. Force est de constater que les jugements quant à l'efficacité relative du traitement expérimental en cours peuvent différer selon les cliniciens-chercheurs. De plus, ce jugement peut évoluer au cours de la conduite d'un essai clinique et placer le clinicien-chercheur devant un dilemme éthique, en particulier lorsque son expérience lui suggère qu'une option thérapeutique est plus avantageuse qu'une autre. Cependant, ce jugement individuel n'a de valeur qu'en référence au consensus de la communauté clinique. Un consensus que le chercheur ne peut qu'imaginer, puisque les données probantes sont en voie d'élaboration. Ainsi, la décision de poursuivre, modifier ou interrompre un traitement expérimental peut difficilement reposer sur le jugement du clinicien-chercheur, ni même de sa communauté de référence. Cette décision se prend sur la base de preuves expérimentales les plus objectives possible, même si elles font ultimement l'objet d'une interprétation.

Les facteurs psychologiques et l'expérience qui constituent le fondement d'une culture clinique spécifique sont déterminants dans le choix des études qui seront menées dans un établissement donné. Une grande diversité d'essais cliniques est proposée pour des indications similaires, permettant à chaque clinicien-chercheur ou équipe de cliniciens-chercheurs de choisir les essais qui correspondent à leurs valeurs et à leur culture clinique spécifique et de refuser ceux qu'ils jugent inacceptables de leur point de vue. Autrement dit, la diversité des contextes cliniques permet la conduite d'une diversité d'essais. Dans ce contexte, la notion d'équilibre clinique est peu susceptible d'être un frein à la tenue d'études.

Nous croyons que la capacité financière du promoteur de l'étude de poursuivre malgré l'incertitude constitue une barrière plus significative. En effet, tant que les toxicités sont contrôlées et qu'il n'y a pas de différence statistiquement significative entre les options thérapeutiques - tant en termes d'efficacité que de sécurité les autorités réglementaires ne s'opposeront pas à la poursuite des essais ou au passage d'une phase d'essai à une autre, et ce, jusqu'à l'obtention de preuves plus solides qui pourront résoudre objectivement l'authentique incertitude qui peut persister dans le traitement d’une condition donnée.

En accord avec Webster et coll. (2016), nous retenons, en vue de notre réflexion sur le rôle du CER, que l'equipoise ne repose pas seulement sur l'information scientifique, mais sur un ensemble de valeurs et de facteurs psychologiques individuels, et qu'elle peut difficilement être un objet de préoccupation éthique au moment du recrutement. Le processus décisionnel objectif par lequel la sécurité des essais est assurée doit faire partie des éléments à partager avec le candidat participant. Quant aux motivations plus contextuelles, nous croyons que ces éléments, bien que potentiellement déterminants dans la gamme d'essais cliniques offerts dans un établissement donné, devraient jouer un rôle secondaire lors du recrutement d'un participant par un clinicien-chercheur ayant une obligation thérapeutique à l'égard d'un patient admissible à une étude. 


\section{La méprise thérapeutique}

Ce concept est-il vraiment éclairant pour bien cadrer éthiquement le premier contact entre médecin-chercheur et patient?

Plusieurs chercheurs en bioéthique soulignent le flou relatif qui caractérise le concept de méprise thérapeutique. La définition généralement admise désigne la confusion chez le patient participant à une étude thérapeutique, entre la dimension recherche et la dimension clinique. Pour Applebaum et coll. (2012), cette confusion peut prendre trois formes observables chez le patient:

- Il entretient des croyances erronées sur l'individualisation des procédures de recherche pour répondre à ses besoins;

- Il surestime les bénéfices de l'étude et sous-estime les risques;

- Il a une mauvaise compréhension des objectifs de la recherche.

Pareilles confusions peuvent venir de la présentation du projet par une équipe de recherche dont le discours est largement clinique ou d'un formulaire de consentement confondant. Tout cela incite à la prudence. Le concept de méprise thérapeutique a le mérite de mettre en relief un enjeu du consentement éclairé, présent dès le premier contact: le risque de mobiliser les espoirs de guérison du patient pour l'inciter à participer à une étude.

Cela dit, la méprise thérapeutique ne constitue pas un critère d'évaluation aussi solide qu'il n'y paraît à première vue. D'une part, il peut être difficile de ne pas confondre les attitudes normales et légitimes du patient que sont l'espoir ou l'optimisme avec une incompréhension de la recherche : les patients optimistes seraient-ils incapables de consentement éclairé? D’autre part, il deviendrait pratiquement impossible de mener des études s'il fallait en exclure tous les patients qui investissent quelque espoir dans la participation à l'étude (Thong et coll., 2016).

À ce propos, certains auteurs proposent de distinguer l'optimisme irréaliste, lié à une mauvaise compréhension des processus de recherche, de l'optimisme dispositionnel qui s'applique à la vie en général (Jansen et coll., 2016). L'optimisme ne constitue pas en soi un problème éthique - sinon l'avenir appartiendrait aux pessimistes! - tant qu'il ne résulte pas d'une confusion chez le participant à propos des conditions de sa participation à l'étude.

Admettons donc que le concept de méprise thérapeutique constitue une judicieuse incitation à la prudence dans l'exercice du pouvoir d'influence du médecin-chercheur. Le patient qui entretient une relation de confiance avec le médecin et qui s'attend à ce qu'il lui offre les soins les mieux adaptés à ses besoins peut effectivement percevoir l'invitation à participer à une étude comme une recommandation de traitement et perdre de vue la dimension recherche du traitement expérimental et les risques qu'il implique. Pour citer l'EPTC 2:

Il n’est pas impossible que les cliniciens suscitent ainsi chez les patients-participants une méprise thérapeutique qui est susceptible d'influer sur le recrutement et le processus de consentement [...]. Aussi doivent-ils veiller à ne pas créer, auprès des participants, des attentes irréalistes concernant les bénéfices potentiels du projet de recherche (EPTC 2, 2014: 172). 
On peut adhérer à ce précepte de l'EPTC 2 indiquant que la thérapie expérimentale ne devrait pas «interférer avec [les] objectifs de soins» (EPTC 2, 2014 : 171). Une difficulté se pose pourtant dans cette problématisation de la méprise thérapeutique. On traite la question comme s'il n'y avait pas d'incertitude ou de risque propre à la pratique clinique. Comme si, pour chaque cas, il y avait une option sûre. Or, ce n'est pas le cas.

Compte tenu du manque de données objectives disponibles, la particularité des options thérapeutiques expérimentales proposées dans le cadre d'essais cliniques randomisés ou non est leur plus grande incertitude ou indétermination, en comparaison avec les options thérapeutiques homologuées. Cependant, cette incertitude est parfois préférable à un pronostic faible ou à une faible efficacité d'un traitement connu. Dans ces situations, en l'absence de données probantes avantageant l'une ou l'autre option, la décision repose essentiellement sur un choix de valeur du patient. Le jugement clinique devient pratiquement celui de l'individu en attente de soins, qu'ils soient standards ou expérimentaux.

Selon certaines études menées auprès d’oncologues (dont une citée par Thong et coll., 2016), la moitié d'entre eux disent recruter leurs patients pour qu'ils bénéficient d'avantages thérapeutiques et non pour produire des connaissances, laissant supposer un possible biais éthique pour l'obtention du consentement. Or les études thérapeutiques ont précisément un double rôle clinique et recherche. Bien que la portée thérapeutique de certaines études puisse être limitée, il faut les juger sur la base des bénéfices qu'elles sont susceptibles d’apporter au patient et pour les risques qu'elles posent.

Prenons pour exemple une étude sur le lupus érythémateux où le patient reçoit un traitement standard en plus d'un traitement expérimental ou d'un placébo. Bien sûr, l'avantage immédiat du médicament à l'étude est plus hypothétique. Cependant, plusieurs patients lupiques sont aux prises avec des symptômes qui nuisent significativement à leur qualité de vie quotidienne et ont souvent connu de nombreux régimes thérapeutiques peu ou pas efficaces et une détérioration de leur état; aussi espéreront-ils tirer un bénéfice de leur participation. L'équipe soignante n'aurait-elle pas une approche similaire du consentement aux soins lors de l'essai d'un nouveau traitement homologué après plusieurs échecs thérapeutiques, en exposant les bénéfices escomptés, mais aussi l'incertitude liée au nouveau traitement? Et la motivation du patient pour accepter le nouveau traitement ne serait-elle pas du même ordre, soit l'espoir de réduire le fardeau de la maladie, nonobstant l'incertitude?

Que retenir de cette analyse sinon que le concept de méprise thérapeutique n'est pas aussi déterminant ou généralisable que souhaité dans l'évaluation éthique des études thérapeutiques, et que chaque option devrait être évaluée en fonction des risques qu'elle pose et des incertitudes qu'elle porte?

Avant d'aborder le rôle du CER à la lumière de cette analyse, nous mettons en relief quelques principes qui nous paraissent déterminants. Les premiers concernent les thérapies expérimentales:

- Les thérapies expérimentales peuvent constituer des options thérapeutiques valables;

- Les approches cliniques standards font l'objet de controverses ou de désaccords dans la communauté clinique;

- L'évaluation éthique des thérapies porte sur les risques, les bénéfices et le degré d’incertitude qui sont présents aussi bien dans les options expérimentales que standards. 
Les secondes portent sur la distinction entre études thérapeutiques et non thérapeutiques. Nous avons traité jusqu'à maintenant des essais thérapeutiques qui ont le potentiel d'apporter des bénéfices aux patients. En matière d'évaluation du risque, ils doivent être clairement distingués des essais non thérapeutiques qui visent la production de connaissances sans bénéfices directs pour les participants. Dans le premier cas, les risques assumés par les participants devraient être proportionnels aux bénéfices qu'eux-mêmes pourraient potentiellement en retirer. Dans le second cas, les risques devraient être relativement faibles en regard des connaissances potentielles. Cette distinction assez classique est reprise par des auteurs récents, dont Westra et De Beaufort (2011). Notons que, dans le cadre de certaines études thérapeutiques, des interventions sont prévues strictement à des fins de recherche. Le risque de ces interventions est alors évalué comme un objectif à visée non thérapeutique.

\title{
Le rôle du CER face aux enjeux éthiques du recrutement
}

Comment la présente analyse des concepts d'équilibre clinique et de méprise thérapeutique éclaire-t-elle le rôle du CER qui examine les enjeux éthiques du recrutement?

Si l'équilibre clinique justifie la tenue d'une étude thérapeutique, en d'autres mots, s'il existe une réelle incertitude quant aux traitements les plus efficaces pour une condition donnée, la thérapie expérimentale devrait être conçue comme faisant partie des options thérapeutiques légitimes. Par conséquent, il ne devrait pas y avoir d'interférence entre les objectifs de recherche et les objectifs de soins. La prise de risque devant être proportionnelle aux bénéfices attendus.

La notion de méprise thérapeutique met en opposition les impératifs de la recherche et de la pratique clinique, alors que le souci de la clinique et de la recherche devraient être complémentaires en contexte d'incertitude thérapeutique. Dans ce contexte de pratique, nous ne croyons pas que la séparation entre les fonctions de recherche et de traitement améliore le processus de recrutement. La nuance apportée par l'article 11.6 de I'ETPC 2 (2014) devient ainsi fondamentale :

\begin{abstract}
En règle générale, on peut réduire la possibilité que les participants ne se méprennent sur l'aspect thérapeutique d'un essai et éviter ainsi de créer un malentendu ou de fausses attentes, si les cliniciens qui dispensent des soins de santé au patient interviennent aussi peu que possible dans le recrutement et le processus de consentement. Idéalement, les fonctions de traitement et de recherche seront assumées par des personnes différentes, mais dans certains cas, il sera dans l'intérêt des participants d'impliquer leur clinicien traitant au processus de recrutement et de consentement.
\end{abstract}

Si nous avons parlé tout au long de notre exposé du médecin-chercheur ou du clinicien-chercheur, c'est précisément que nous doutions de la pertinence de lui substituer un intervenant dont la seule fonction serait d'assurer le recrutement et d'accompagner le participant potentiel dans le processus de consentement. D'une part, il y a fort à parier que le patient va de toute façon se tourner vers son médecin traitant pour obtenir son conseil. D’autre part, il faut prendre acte de la diversité d'appréciation des traitements standards par les médecins.

Nous sommes d'avis que la meilleure approche éthique de la méprise thérapeutique consiste à mettre au premier plan cet autre passage de l'article 11.6 de l'EPTC 2 (2014) : 
Les CER et les chercheurs qui mènent des essais cliniques seront attentifs au phénomène de méprise thérapeutique lié aux essais cliniques. Aussi veilleront-ils à ce que les démarches de recrutement et de sollicitation du consentement mettent clairement l'accent sur les éléments particuliers de la participation à l'essai clinique qui sont essentiels à la recherche, ainsi que sur les différences entre le projet de recherche dont il s'agit et les soins cliniques habituels que les participants pourraient recevoir dans un autre contexte.

Nous remplacerions toutefois les mots «dans un autre contexte» par «dans le contexte particulier de cette étude et du traitement de ce patient». En ce sens, le travail du CER consiste à entrer en dialogue avec le médecin-chercheur pour connaître son expérience clinique, son appréciation des traitements standards, ses critères de sélection des patients. C'est identifier les paramètres qui vont conditionner son discours auprès du patient, afin de le sensibiliser aux biais possibles.

Bien que l'examen du CER procède selon des lignes directrices éthiques reconnues, que ses analyses soient critériées et ses conclusions rationnellement justifiées, nous devons poser une limite à l'action du CER : il n'a pas à sonder les reins et le cœur du chercheur, à le soumettre au polygraphe pour connaître ses valeurs et ses raisons d'agir. Dans ses échanges avec le CER, le chercheur peut exprimer les valeurs qui l'animent et il ne nous paraît pas scandaleux que les membres du CER les partagent.

Le dialogue entre le CER et le chercheur sera l'occasion d'aborder l'attitude éthique idéale du chercheur clinicien : humilité, scepticisme et rigueur scientifique, mais aussi adhésion au devoir de soigner. Ces valeurs sont identifiées notamment par Shamy et coll. (2014). Nous ajoutons : écoute, ouverture et transparence, sans oublier le tact nécessaire à bien faire comprendre et sentir au patient le risque inhérent à la recherche, sans annihiler sa confiance dans une possible issue thérapeutique positive. Bref, travailler à réduire les faux espoirs sans tuer l'espoir. 


\section{Bibliographie}

Allmark, P. et Mason, S. (2006). Should desperate volunteers be included in randomised controlled trials? Journal of Medical Ethics, 32 (9), 546-553.

Appelbaum, P. S., Anatchkova, M., Albert, K., Dunn, L.B. et Lidz, C. W. (2012). Therapeutic misconception in research subjects: Development and validation of a measure. Clinical Trials, 9, 748-761.

Blumenthal-Barby, J. S. S. (2011). On the utility and distinctness of the concept of behavioral equipoise. The American Journal of Bioethics, 11 (2), 9-19.

Burke, N. J. (2014). Rethinking therapeutic misconception: social justice, patient advocacy, and cancer clinical trial recruitment in the US safety net. BMC Medical Ethics, 15, 68-74.

Conseil de recherches en sciences humaines du Canada, Conseil de recherches en sciences naturelles et en génie du Canada, Instituts de recherche en santé du Canada (décembre 2014). Énoncé de politique des trois Conseils : Éthique de la recherche avec des êtres humains. (cité dans le texte comme EPTC2) http://www.ger.ethique.gc.ca/pdf/fra/eptc2-2014/EPTC_2_FINALE_Web.pdf.

Freedman B. (1987). Equipoise and the ethics of clinical research. N Engl J Med., 317, 141-145. https://www.nejm.org/doi/full/10.1056/NEJM198707163170304

Fried C. (1974). Medical Experimentation: Personal Integrity and Social Policy. New York: American Elsevier.

Gelfand, S., (2013). Clinical equipoise: actual or hypothetical disagreement? Journal of Medicine and Philosophy, 38, 590-604.

Jansen, L. A., Mahadevan, D., Appelbaum, P. S., Klein, W. M. P., Weinstein, N. D., Mori, M., Daffé, R. et Sulmasy, D. (2016). Dispositional optimism and therapeutic expectations in early-phase oncology trials. Cancer, 122 (8), 1238-1246.

Instituts de recherche en santé du Canada, Conseil de recherches en sciences naturelles et en génie du Canada, Conseil de recherches en sciences humaines du Canada. (1998, avec les modifications de 2000, 2002 et 2005). Énoncé de politique des trois Conseils : Éthique de la recherche avec des êtres humains. (cité dans le texte comme EPTC). http://www.ger.ethique.gc.ca/archives/tcps-eptc/docs/TCPS\%20octobre\%20 2005_F.pdf

Lidz, C. W., Albert, K., Appelbaum, P., Dunn, L. B., Overton E. et Pivovarova, E. (2015). Why is therapeutic misconception so prevalent? Cambridge Quarterly of Healthcare Ethics, 24, 231-241.

Malmqvist, E. (2011). (Mis)understanding exploitation. IRB: Ethics \& Human Research, 33 (2), 1-5.

McCormack, P. et Woods, S. (2012). The therapeutic misconception, decision-making and informed consent: the ethics of trial participation for neuromuscular disorders. Neuromuscular Disorders, 22, 191-192.

Schlichting, D. E. (2010). Destabilizing the "equipoise" framework in clinical trials: prioritizing non-exploitation as an ethical framework in clinical research. Nursing Philosophy, 11, 271-279.

Scott, J. (2013). Therapeutic misconceptions and misestimations in oncology: a clinical trial nurse's guide. Clinical Journal of Oncology Nursing, 17 (5), 486-489.

Shamy, M. C. F., Stahnisch, F. W. et Hill, M. D. (2014). Fallibility: a new perspective on the ethics of clinical trial enrollment. International Journal of Stroke, 10, 2-6.

Thong, I. S. K., Foo, M. Y., Sum, M. Y., Capps, B., Lee, T.-S., Ho, C. et Sim, K. (2016). Therapeutic misconception in psychiatric research: a systematic review. Clinical Psychopharmacology and Neuroscience, 14 (1), 17-25. 
Webster, F., Weijer, C., Todd, L., Grimshaw, J. M., Marshall, A. P., Cook, D., MacLennan, G., Cuthbertson, B. H. et Francis, J. J., (2016). The ethics of future trials: qualitative analysis of physicians' decision making. Trials, $17,12-20$.

Weijer, C. et Miller, P. B., (2003). Therapeutic obligation in clinical research. Hasting Center Report, Mai-Juin, 3.

Westra, A. E. et De Beaufort, I. (2011). The merits of procedure-level risk-benefit assessment. IRB: Ethics \& Human Research, 33 (5), 7-13.

Woods, S., Hagger, L. E. et Mccormack, P. (2014). Therapeutic misconception: hope, trust and misconception in paediatric research. Health Care Annals, 22, 3-21. 
TITRE: RECRUTEMENT EN PHASE 1 CHEZ DES PARTICIPANTS ATTEINTS D'UNE MALADIE GRAVE

Auteur(s): François POULIOT

PublicAtION: RECRUTEMENT ET CONSENTEMENT À LA RECHERCHE : RÉALITÉS ET DÉFIS ÉTHIQUES

PAGES: $65-71$

ISBN: 978-2-7622-0357-8

Directeurs: Ana Marin, BéATrice Eysermann et Michel T. GIROUX

URI: HTTP://HDL.HANDLE.NET/11143/14109

DOI: HTTPS://DOI.ORG/10.17118/11143/14109 


\section{Recrutement en phase 1 chez des participants atteints d'une maladie grave}

François Pouliot, M.D., Ph. D., vice-président du Comité d'éthique de la recherche du CHU de Québec-Université Laval

Résumé : Si les noms TeGenero et Bial sont associés à des tragédies chez des volontaires sains, ils ne doivent pas faire oublier les problèmes plus fréquents et tout aussi sérieux chez les participants atteints d'une maladie grave comme le cancer. La différence fondamentale est qu'il n'existe pour les volontaires sains aucun intérêt thérapeutique à participer à une telle recherche.

La première utilisation de nouvelles molécules chez l'humain, appelée couramment phase 1, cherche à étudier la sécurité du produit et à établir la dose optimale. En effet, l'identification de cibles moléculaires précises, la fabrication de molécules sur mesure, le phénomène de résistance, l'urgence d'offrir une certaine alternative à des patients en fin de course incitent à utiliser le nouveau produit plus rapidement et auprès d'une plus large population. Si l'on a pu accéder rapidement aux nouveaux médicaments contre le SIDA, on devrait faire de même contre le cancer.

Au plan éthique, plusieurs voient un intérêt thérapeutique pour tout malade participant à la recherche, même lors de la toute première utilisation. Si les bénéfices sont habituellement peu importants, le rapport risques/ bénéfices est souvent intéressant. D'ailleurs, la motivation première de ces participants est le plus souvent l'amélioration de leur situation, non la production de connaissances. Plus radicalement encore, des patients sont prêts à prendre de grands risques pour la recherche sur leur maladie ou d'autres problèmes. Au regard de leur autonomie et de leur capacité à consentir, la prudence des autorités concernées apparaît à certains patients comme une forme dépassée de paternalisme.

Mots-clés : recherche, phase 1, participants, autonomie, paternalisme 


\section{Introduction}

En 2006, une jeune compagnie nommée TeGenero fit la manchette des médias parce que six participants à la recherche se retrouvèrent aux soins intensifs à la suite de l'utilisation d'une substance modifiant les réactions immunitaires du corps humain. L'ensemble des volontaires avaient reçu leur dose de médicament à peu près en même temps, ce qui avait empêché les chercheurs de prévenir les mêmes effets secondaires chez les participants ${ }^{1}$. En 2015, la compagnie Bial reçut le même genre de publicité quand plusieurs participants en bonne santé présentèrent des symptômes graves après une première dose d'un nouveau produit². Ces deux tragédies ne doivent pas faire oublier des problèmes plus fréquents et tout aussi sérieux qui se retrouvent chez des participants atteints de maladies graves comme le cancer ou la sclérose en plaques. La différence fondamentale entre ces deux groupes est que les volontaires sains n'ont aucun intérêt thérapeutique à participer à une telle recherche.

La toute première utilisation de nouvelles molécules chez des patients gravement malades est particulièrement présente en oncologie. Au MD Anderson Cancer Center, par exemple, environ 800 personnes atteintes de cancer parmi les 11000 participants à la recherche sont inscrites dans des projets de phase 1 répartis en 110 projets $^{3}$. Ces projets sont souvent des thérapies moléculaires ciblées (Molecular Targeted Therapies) visant des récepteurs cellulaires spécifiques.

La question est d'une grande actualité et représente un problème éthique significatif. N'est-ce pas exploiter des personnes grandement vulnérables, prêtes à accepter tout projet de recherche, même risqué, au nom de la science alors que le désir de vivre les pousse aussi à le faire? Des professionnels de la santé et des chercheurs consciencieux peuvent-ils laisser à des personnes non expertes l'évaluation des risques et des bénéfices d'un tel projet de recherche? De quel droit veut-on imposer à ces personnes un certain niveau de risque acceptable alors qu'elles sont prêtes à prendre plus de risques?

\section{Le développement d'une nouvelle molécule}

Le développement d'une nouvelle molécule est un long et complexe processus. Après les recherches fondamentales en laboratoire puis sur les animaux arrive le moment où le produit montre suffisamment d'intérêt pour être testé chez l'humain pour la première fois. Cette étape, appelée phase 1, cherche à étudier la sécurité du produit, à établir la dose optimale (quantité, fréquence et voie d'administration) et à documenter les premiers signes d'efficacité. Une telle étude compte entre 15 et 30 participants, regroupés en petites cohortes de trois à cinq personnes.

Quand les résultats de cette première étape le permettent, la phase 2 est enclenchée. Celle-ci continue à récolter les données de sécurité, mais vise à vérifier si le nouveau médicament a une certaine efficacité quant au problème étudié. Elle compte souvent moins de 100 participants.

La phase 3 veut comparer l'efficacité du nouveau produit avec celle du traitement considéré comme standard. Des centaines, voire des milliers de participants sont recrutés pour une telle recherche. Quand tout se passe

1. Voir http://www.nytimes.com/2006/04/07/world/europe/when-drug-trials-go-horribly-wrong.html

2. Le journal Le Figaro a publié une série d’articles sur le groupe Bial. Voir par exemple : Essai thérapeutique. Bial assure avoir respecté "les bonnes pratiques», dans l'édition du 15 janvier 2016, ou encore Rennes. L'agence du médicament publie le protocole de Bial, dans celle du 22 janvier 2016.

3. Cf. Messenger, MD Anderson Cancer Center, Houston, July/August 2010, p. 5. 
bien, cette étape aboutit à une approbation des autorités en la matière, notamment Santé Canada, et à la commercialisation du produit.

Enfin, la phase 4 survient après cette commercialisation. Elle cherche à examiner de nouvelles indications possibles. Des informations sur la sécurité acquises auprès des utilisateurs du produit sont collectées.

\section{Quelques enjeux éthiques liés à la participation des personnes atteintes d'une maladie grave aux recherches de phase 1}

\section{Le recrutement de participants malades}

La règle générale veut que la recherche de phase 1 se fasse avec des volontaires sains. Parfois, celle-ci n'est pas possible à cause de la spécificité du produit à l'étude (par exemple, un anticorps dirigé contre des protéines anormales retrouvées dans les cellules cancéreuses) ou encore de ses graves dangers contre l'organisme (effets de la thalidomide et de ses dérivés sur le développement intra-utérin). II n'en reste pas moins que, dans la majorité des cas, la sécurité du produit n'est pas établie tant qu'il n'est pas utilisé chez l’humain.

Le recrutement de participants malades est donc souvent nécessaire, mais il fait l'objet de restrictions importantes. Dans la Déclaration d'Helsinki de l'Association médicale mondiale (AMM, 2013) sur les principes éthiques applicables à la recherche médicale impliquant des êtres humains, la priorité a été accordée à la protection des participants. La Déclaration en fait un devoir du médecin :

4. Le devoir du médecin est de promouvoir et de sauvegarder la santé, le bien-être et les droits des patients, y compris ceux des personnes impliquées dans la recherche médicale. Le médecin consacre son savoir et sa conscience à l'accomplissement de ce devoir [...].

9. Il est du devoir des médecins engagés dans la recherche médicale de protéger la vie, la santé, la dignité, l'intégrité, le droit à l'autodétermination, la vie privée et la confidentialité des informations des personnes impliquées dans la recherche. La responsabilité de protéger les personnes impliquées dans la recherche doit toujours incomber à un médecin ou à un autre professionnel de santé et jamais aux personnes impliquées dans la recherche même si celles-ci ont donné leur consentement.

On a ainsi voulu préserver une population vulnérable et non experte contre les investigateurs et contre ellemême. Selon la Déclaration d'Helsinki (AMM, 2013), le simple fait d'appartenir à un groupe vulnérable, que ce soit au plan physique, psychologique, social ou économique, implique que la recherche doit répondre aux besoins ou aux priorités sanitaires de ce groupe. Ce groupe devrait aussi bénéficier des connaissances, des pratiques ou interventions qui en résultent (AMM, 2013, art 20). Le médecin peut aussi recourir à une intervention non avérée si elle offre une chance de sauver la vie, rétablir la santé ou alléger les souffrances du patient quand il n'y a pas d'intervention avérée («traitement standard») ou faute d'efficacité de ces interventions (AMM, 2013, art. 37).

Or, de plus en plus, les participants eux-mêmes veulent fixer le niveau de risque acceptable par rapport aux bénéfices attendus en fonction de leurs objectifs et valeurs. Aux États-Unis, l'influence du groupe de pression ACT UP sur le développement de la trithérapie contre le SIDA est un bon exemple de l'impact de patients devenus activistes. Alors que les organismes de régulation des médicaments voulaient s'assurer de la sécurité maximale des nouvelles substances, ces groupes insistaient pour avoir accès rapidement à celles-ci dès qu'elles montraient un bénéfice significatif. Des regroupements de patients ont aussi fait pression sur la FDA 
afin d'accélérer l'accès à l'Irinotecan, un médicament prometteur contre le cancer du côlon (Daugherty, 1997; Wachter, 1992; Rothenberg, M.L, Eckart, J.R., Kahn, J.G. et coll., 1996). Ces exemples montrent que certains participants contestent une certaine forme de paternalisme voulant les protéger contre eux-mêmes et réclament de pouvoir choisir eux-mêmes le niveau de risque jugé acceptable. Un tel conflit entre obligation professionnelle et autodétermination ne trouvera de solution éthiquement acceptable qui si les deux groupes évoluent vers un partenariat qui tiendra compte des risques et des bénéfices établis par la science et qui veillera méticuleusement à respecter le processus de consentement.

\section{Le rapport entre les risques et les bénéfices}

La volonté de participer à un essai clinique ne peut suffire pour y avoir accès. Il doit exister une forme de proportion ou d'équilibre qui justifie l'utilisation d'un tel traitement. La Déclaration indique, à l'article 16, qu'une «recherche médicale impliquant des êtres humains ne peut être conduite que si l'importance de l'objectif dépasse les risques et inconvénients pour les personnes impliquées». Le Code civil du Québec affirme, à l'article 20, qu'une «personne majeure, apte à consentir, peut participer à une recherche susceptible de porter atteinte à son intégrité pourvu que le risque couru ne soit pas hors de proportion avec le bienfait qu'on peut raisonnablement en espérer».

Le Code civil est cependant plus restrictif pour les mineurs et les majeurs inaptes, la recherche devant laisser espérer un bienfait ou des résultats bénéfiques4

Les études de phase 1 peuvent avoir un impact positif sur la santé des participants. Les données quantitatives compilées par Agrawal et Emanuel (Agrawal et Emanuel, 2008) montrent des résultats globaux modestes dans le domaine de l'oncologie : on parle de réponse dans 4,4 \% des cas : une réponse complète dans 1,9\% des cas, une réponse partielle (correspondant à au moins $50 \%$ de diminution de la tumeur) dans 2,5\% des cas. Cependant, certaines réponses spectaculaires ont été notées : le cancer médullaire de la thyroïde avec le XL184; le cancer de l'ovaire avec le patupilone; le sarcome d'Ewing avec des inhibiteurs de l'IGFR; la maladie de Castleman avec l'anti-interleukine-6; le cancer des testicules avec le cisplatine (une réponse a été notée dans $50 \%$ des cas, une guérison dans $25 \%$ ); la leucémie myéloïde chronique avec le Gleevec (une réponse hématologique a été notée dans $98 \%$ des cas).

Du côté des risques, la même source parle d'une mortalité à 0,5\% et d'une toxicité importante (niveaux 3 et 4) chez $10 \%$ des participants à des études de phase 1 en oncologie.

Ces chiffres ne prennent évidemment pas en compte les aspects qualitatifs de la survie liés à la qualité bonne ou mauvaise de la vie, aux bienfaits psychologiques et sociaux tirés de la participation à un projet de recherche, au sentiment de mieux contrôler l'évolution de la maladie, de contribuer à l'évolution de la science et à l'amélioration des soins à venir, pour ne nommer que quelques éléments. Il en va souvent du sens donné à ce qui leur reste de vie (Jansen, Appelbaum, Klein et coll., 2011; Daugherty, Ratain, Grochowski et coll. 1995).

\footnotetext{
4. «Un mineur ou un majeur inapte ne peut participer à une recherche susceptible de porter atteinte à son intégrité qu'à la condition que le risque couru, en tenant compte de son état de santé et de sa condition personnelle, ne soit pas hors de proportion avec le bienfait qu'on peut raisonnablement en espérer. Il ne peut, en outre, participer à une telle recherche qu'à la condition que la recherche laisse espérer, si elle ne vise que lui, un bienfait pour sa santé ou, si elle vise un groupe, des résultats qui seraient bénéfiques aux personnes possédant les mêmes caractéristiques d’âge, de maladie ou de handicap que les membres du groupe.» Code civil du Québec, art. 20.
} 


\section{Le consentement libre et éclairé}

L'étude déjà ancienne de Daugherty et coll. (1995) montrait que les participants en phase 1 sont motivés par la perspective thérapeutique, peu par l'avancement de la science et l'altruisme. Ils ont une perception correcte des risques et des bénéfices, mais méconnaissent le rôle des études de phase 1 dans l'établissement de la dose adéquate. Il y a donc raison d'être prudent quand on propose une étude de phase 1 à des gens vulnérables. Chaque étape doit être réalisée avec grande attention.

Dans le cas où ces patients sont traités dans des unités de soins intensifs ou reçoivent des soins palliatifs, les mesures de précaution seront encore plus importantes. Leur état physique et mental, de même que les conditions très concrètes dans lesquels ils sont placés peuvent interférer avec la possibilité concrète de la recherche. Il est difficile de recevoir l'information pertinente, de réfléchir de façon sereine selon ses valeurs, de communiquer sa décision, sans parler de la réalisation sécuritaire de l'étude elle-même, quand la chambre est pleine d'appareils, que le va-et-vient du personnel dérange ou, au contraire, quand les lieux n'accueillent aucune technologie! Aussi surprenant que cela puisse paraître de prime abord, ils ne sont pas automatiquement exclus d'une participation à une étude de phase 1.

\section{Conclusion}

Dans l'état actuel, il est possible pour des patients atteints d'une maladie grave, mais dans un état fonctionnel relativement bon, de participer à des études de phase 1. Parfois, ce sont les seuls qui peuvent être recrutés. Certains veulent aller loin et sont prêts à prendre des risques importants en participant à ces études de phase 1 . Les avantages et les risques leur ont été expliqués et ils ont été jugés aptes à consentir. Au regard de leur autonomie et de leur capacité à consentir, le refus des autorités concernées à les recruter apparaît à certains comme une forme dépassée de paternalisme. Devrait-on refuser une telle participation uniquement quand l'objet de l'étude n'est pas en lien avec le problème de santé dont ils souffrent? Ne devrait-on pas aussi retenir ceux qui «offrent leurs corps à la science», prêts à recevoir tout ce qu'on leur proposera? Tel semble bien être l'état des lieux en ce moment. 


\section{Bibliographie}

Agrawal, M. et Emanuel, E.J. (2008). Phase 1 Oncology Research. Dans Emanuel, E.J. et coll., The Oxford Textbook of Clinical Research Ethics (p. 356-366). Oxford University Press.

Anderson, J.A. et Kimmelman, J. (2010). Extending Clinical Equipoise to Phase 1 Trials Involving patients : Unresolved Problems, Kennedy Institute of Ethics Journal, 20 (1), 75-98.

Association médicale mondiale (AMM). (2013). Déclaration d'Helsinki de l'AMM-Principes éthiques applicables à la recherche médicale impliquant des êtres humains, Fortaleza.

Code civil du Québec, RLRQ, c. C.c.Q.-1991.

Daugherty, C., Ratain, M.J., Grochowski, E. et coll. (1995). Perceptions of Cancer Patients and Their Physicians involved in Phase I Trials. J Clin Oncol., 13, 1062-1072.

Daugherty, C, Siegler M, Ratain, M.J. et Zimmer, G (1997). Learning from Our Patients: One Participant's Impact on Clinical Trial Research and Informed Consent, Annals of Internal Medicine, 125, 892-897.

Jansen, L.A., Appelbaum, P.S., Klein, W.M.P. et coll. (2011). Unrealistic Optimism in Early-Phase Oncology Trials, IRB: Ethics and Human Research, 33, 1-8.

Rothenberg, M.L., Eckart, J.R., Kahn, J.G. et coll. (1996). Phase II trial of irinotecan in patients with progressive or rapidly recurrent colorectal cancer, J Clin Oncol.,14, 1128-35.

Wachter, R.M. (1992). AIDS, activism, and the politics of health, N Engl J Med., 326, 128-33. 
TITRE: À QUOI UNE PERSONNE SOUFFRANT D'UN TROUBLE MENTAL CONSENT-ELLE DANS UN CONTEXTE DE RECHERCHE?

Auteur(s): JACQUES QUINTIN

Publication: ReCRUtEMENT ET CONSENTEMENT À LA RECHERCHE : RÉALITÉS ET DÉFIS ÉTHIQUES

PAGES: $72-85$

ISBN: 978-2-7622-0357-8

Directeurs: Ana Marin, BÉAtrice Eysermann et Michel T. GIROUX

URI: HTTP://HDL.HANDLE.NET/11143/14110

DOI: HTTPS://DOI.ORG/10.17118/11143/14110 


\section{À quoi une personne souffrant d'un trouble mental consent-elle dans un contexte de recherche?}

Jacques Quintin, philosophe, professeur au département de psychiatrie, Faculté de médecine et des sciences de la santé, Université de Sherbrooke

Résumé : L'histoire de la psychiatrie regorge d'expériences douteuses qui ont entraîné des souffrances chez les participants à la recherche, souvent sans leur consentement. Si ces enjeux éthiques entourant le consentement paraissent évidents, il n'en demeure pas moins que l'obtention d'un consentement libre et éclairé et la préparation des cliniciens et des chercheurs à la pensée réflexive ne vont pas de soi. Dans un tel contexte, il devient difficile de déterminer objectivement un agir éthique. Après un détour historique et un regard phénoménologique sur la maladie mentale et la prise de décision, il ressort de cela que les personnes appuient leur choix sur ce qui fait sens pour eux, et que le sens se dégage dans un dialogue. Une démarche dialogique est proposée afin de se protéger contre toute motivation unilatérale. Des considérations pragmatiques sont proposées pour contextualiser le dialogue.

Mots clés : Consentement, sens, dialogue, phénoménologie, aptitude

Abstract: The history of psychiatry abounds in doubtful experiments which entailed sufferings at the participants in research, often without their consent. If these ethical stakes surrounding the consent seem obvious, the fact remains that the obtaining of a free consent and the preparation of the clinicians and the researchers to reflexive thought is not obvious. In such a context, it becomes difficult to determine objectively an ethical action. After a historical detour and a phenomenological look at mental illness and decision-making process, it appears that people make their choice on what is meaningful, and that meaning appears in dialogue. A dialogical approach is proposed to protect oneself against any unilateral motivation. Pragmatic considerations are suggested for contextualizing the dialogue.

Keywords: Consent, meaning, dialogue, phenomenology, capacity 


\section{Introduction}

Le champ de la psychiatrie est très fertile à propos des questions éthiques qui entourent les pratiques de la recherche et de la clinique. Dans le champ de la recherche, il existe un souci de protéger les personnes vulnérables en raison de leur état de santé. Toutefois, ce souci entre souvent en tension avec les intérêts des chercheurs à développer de nouvelles connaissances justement pour diminuer la vulnérabilité des personnes souffrant d'un trouble mental (Roberts, 2002). Rhodes (2005) va jusqu'à proposer que l'obtention d'un consentement à la participation à une recherche ne devrait pas être un absolu pour des raisons d'équité dans la mesure où c'est la population entière qui risque d'être privée de l'avancée des connaissances. De plus, l'histoire de la psychiatrie regorge d'expériences douteuses sur le plan scientifique qui ont entraîné des souffrances chez ceux qui participent à la recherche - souvent sans leur consentement - et qui soulèvent encore aujourd'hui de l'indignation dans la sphère publique (Lehrman et Sharav, 1997).

Même si ces enjeux scientifiques et éthiques paraissent évidents pour l'ensemble de la population, il n'en demeure pas moins que préparer les cliniciens et les chercheurs à la pensée réflexive n'est pas une chose aussi facile qu'on veut bien le croire. Cette tâche exige une meilleure compréhension de l'histoire et des éléments philosophiques et éthiques de la recherche en psychiatrie impliquant des personnes aux prises avec un trouble mental (Roberts et coll., 1998).

Le texte qui suit s'appuie sur deux sources : l'une comme ancien clinicien et l'autre comme chercheur. En tant que clinicien, j'ai pu accompagner, pendant plusieurs années, des personnes souffrant d'un trouble de santé mentale dans un contexte de soins offerts par divers services externes. C'est dans ce contexte d'intervention que je fus fréquemment interpellé pour identifier des personnes qui pourraient participer à une recherche ayant pour objet différentes problématiques liées à la santé mentale. Avant de consentir à la recherche, les personnes malades s'informaient auprès de leur professionnel de la santé afin de mieux comprendre de quoi il s'agissait. C'est à ce moment qu'elles partageaient leur souci, leur malaise ou leur enthousiasme. Il est rapidement apparu que l'obtention d'un consentement à une recherche pour des personnes aux prises avec une maladie mentale ne va pas de soi. Dans un tel cas, le chercheur, comme le clinicien, est confronté à des questions de limite: Jusqu'où peut-il insister, c'est-à-dire jusqu'où doit-il préciser les éléments qui entourent la recherche proposée pour s'assurer que le refus soit bien éclairé? Jusqu'où doit-il se montrer convaincant soit pour rassurer soit pour montrer l'importance de la recherche, d'autant plus qu'insister trop pourrait compromettre l'alliance thérapeutique? Il devient très difficile, dans un tel contexte, de déterminer objectivement un agir éthique.

De la même façon, il est difficile de statuer si un consentement est vraiment libre et éclairé. Qu'en est-il alors du consentement? Est-ce que le consentement permet de tout faire? Ne nous arrive-t-il pas de consentir malgré notre désaccord de principe, de consentir en dépit de nos valeurs? Est-ce que l'obtention d'un consentement est une exigence strictement légale? Si c'est une exigence éthique, est-ce que le respect des préférences et des désirs est suffisant pour justifier une démarche d'un point de vue éthique? Est-ce que le respect de l'autodétermination dans le choix des préférences et des désirs est suffisant? Encore faut-il que la personne démontre des aptitudes à la prise de décision éclairée. Déterminer la capacité d'une personne souffrant d'un trouble de santé mentale à comprendre les risques et les bénéfices d'une participation à une étude clinique demeure un enjeu important pour la communauté des chercheurs en psychiatrie. Le consentement est donc une question importante, aussi bien pour les éthiciens et les cliniciens que pour les chercheurs. 


\section{Historique}

Le phénomène du consentement, plus spécifiquement celui du consentement en recherche pour les sujets souffrant d'une maladie mentale, comme tous les phénomènes, a une origine et une histoire. Cependant, cette histoire du consentement en psychiatrie dans un contexte de recherche ne se distingue pas de celle de la médecine ni de celles concernant les enfants, les personnes handicapées, les personnes âgées, les personnes en situation de soins d'urgence ou dans une situation économique désavantagée. Dans chaque cas, les chercheurs s'appuient sur les mêmes principes et sur le concept de vulnérabilité.

Lorsqu'un phénomène interpelle le devenir humain, il devient impérieux de saisir son origine, son histoire, voire de comprendre ses présupposés, même si l'obligation de l'obtention du consentement est bien établie chez les chercheurs dans les comités d'éthique de la recherche et dans les institutions. Depuis que les médecins s'engagent solennellement au serment d'Hippocrate (Hippocrate, 1994), il est attendu que les médecins agissent dans l'intérêt supérieur du patient, ce qui leur donne une autorité morale. Corrélativement, les patients ont développé une forte confiance en leur médecin.

Au XIXe siècle, un chercheur comme Claude Bernard (2008) écrit en 1865 que les chercheurs peuvent effectuer des recherches sur des sujets humains si celles-ci n'entraînent pas de souffrances ou d'inconvénients chez ces derniers. Au début du XXe siècle (1914), est apparue aux États-Unis une jurisprudence sur le consentement aux soins liée à la reconnaissance judiciaire de l'autonomie de la personne (Ringuet, 2010). En 1931, le Conseil de la santé du Reich de la République de Weimar émet un ensemble de «directives concernant les nouveaux traitements médicaux et l'expérimentation scientifique sur l'homme» affirmant la nécessité du consentement éclairé du patient (Ambroselli, 1988 : 28). En dépit de ces avancées théoriques en faveur de l'obtention du consentement éclairé, celles-ci ont eu peu d'impacts concrets (Faden et Beauchamp, 1986). Le dévoilement des expériences qui se sont déroulées dans les camps nazis durant la Seconde Guerre mondiale montre qu'énoncer des grandes valeurs n'est pas suffisant pour protéger l'être humain contre l'être humain.

C'est la raison pour laquelle, plus près de nous, est apparu le Code de Nuremberg (1947), établi par des juges américains, qui, après ce qui s'est déroulé dans les camps de concentration, affirme que «le consentement volontaire du sujet humain est absolument essentiel» (Ambroselli, 1988 : 104), car la science ne doit jamais transformer ou considérer l'être humain comme un instrument ou, comme le dit Kant, comme un moyen. Ce principe du consentement volontaire est l'objet du premier article sur les dix concernant l'expérimentation sur des sujets humains dans ce code. Au Québec, ce principe apparaît aussi très rapidement dans le Code civil (l'article 11). Si le Code de Nuremberg a exercé une plus grande influence, il n'en demeure pas moins qu'il a rapidement montré ses limites (Faden et Beauchamp, 1986). De là est apparue la Déclaration d'Helsinki (AMM, 1964), qui précise les conditions de la recherche clinique à but thérapeutique et à but scientifique. Suivra le rapport Belmont, publié en 1979. Plus près de nous, en 1998, il y a l'Énoncé de politique des trois Conseils: éthique de la recherche avec des êtres humains (EPTC, 1998).

Pourtant, en dépit de ces progrès en faveur de la protection des personnes, on a fait des recherches sur des prisonniers et sur des patients sans leur consentement. Par exemple, un article de Beecher (1966) révèle qu'il y a eu, dans les années 60, dans un hôpital new-yorkais, le scandale d'une recherche auprès de patients auxquels on a injecté des cellules cancérogènes sans leur consentement. Ces chercheurs agissaient en vue du progrès de la science pour le bien de l'humanité. C'était à peu près la seule valeur qui guidait leur agir. 
Ce n'est que vers la fin des années 1990 que débutèrent des études empiriques sur la capacité de consentement des schizophrènes à la recherche (Appelbaum, 2000; Kovnick et coll., 2003; Capdevielle et coll., 2009). Avant ces recherches, les chercheurs comme les professionnels de la santé acceptaient facilement l'idée que les personnes souffrant d'un trouble lié à la schizophrénie pouvaient difficilement exercer un jugement libre et éclairé en raison de leur état mental et de ses conséquences. La maladie mentale venait compromettre la capacité à délibérer. À l'évidence, la personne souffrant d'un trouble de santé mentale peut transformer les informations en raison d'activités délirantes ou hallucinatoires (Bouaziz et coll., 2011). Cependant, les nouvelles recherches démontrent que la capacité à la délibération n’est pas altérée chez cette population (Capdevielle et coll., 2009), sauf indication contraire. Une personne sera jugée apte à consentir même si elle est aux prises avec un délire si celui-ci n’interfère pas avec sa délibération.

C'est pourquoi nous mettons en garde contre tout agir qui repose sur une seule perspective et sur un minimum de valeurs en ignorant d'autres perspectives et valeurs qui peuvent être en jeu. C'est pour cette raison que je propose une démarche dialogique qui sert de base pour un jugement éclairé, autant pour la personne malade que pour le chercheur. Il s'agit d'établir un dialogue entre toutes les personnes concernées, mais aussi entre différentes valeurs qui sont interpellées dans un tel contexte de recherche. L'une de ces valeurs est justement l'autonomie du patient, qui est la source du consentement. Mais qu'en est-il de cette autonomie chez des patients qui souffrent de maladie mentale?

\section{Un regard phénoménologique}

Le consentement, depuis le Code de Nuremberg, s'établit sur l'information factuelle que le chercheur donne et que le sujet de recherche reçoit. De fait, l'idée du consentement en sciences médicales a été pensée sur le modèle de la science qui sépare l'observateur de l'objet observé afin d'atteindre la plus grande objectivité possible. Pourtant, l'obtention d'un consentement ne relève pas strictement de la science et du discours objectif, mais de la pratique clinique. Dans ce texte, je suggère que la phénoménologie peut nous fournir un nouveau langage, mieux adapté à la rencontre clinique. La phénoménologie, en mettant l'accent sur l'expérience vécue, et non sur le traitement de l'information, ouvre sur le sens : le sens de la recherche scientifique, le sens d'une participation à la recherche, le sens de la maladie et de la santé. La phénoménologie ne vise pas à éliminer le langage de la recherche biomédicale, mais à proposer un nouveau langage qui ouvre pour le sujet sur différentes manières d'interpréter son engagement dans le monde. Il s'agit pour la phénoménologie de mettre en lumière les présupposés qui organisent l'expérience vécue de la personne à qui les chercheurs demandent de donner un consentement.

Selon une approche phénoménologique de la dépression (Ratcliffe, 2015) et de la schizophrénie (Stanghellini, 2004), on observe chez les personnes qui en sont atteintes une difficulté dans les relations interpersonnelles et une difficulté à se projeter dans le temps. Les personnes atteintes de dépression et de schizophrénie se sentent séparées des autres personnes et leur monde est appauvri. Elles se sentent vulnérables. Elles ne voient généralement pas l'intérêt de participer à une recherche qui concerne les autres dans un futur inaccessible. Le chercheur est confronté à l'indifférence et à l'hostilité.

Pour le patient, principalement dans un contexte de recherche qualitative, consentir à ce que le chercheur propose, c'est parfois consentir indirectement à être pris comme un objet. C'est Sartre (1943) qui dit que nous faisons l'expérience de notre vie comme objet dans le regard de l'Autre. Ne parle-t-on pas d'objet d'étude? Déjà, en raison de la maladie, la personne apparaît à elle-même comme un objet (Toombs, 1993). La personne se 
vide de sa substance, ne reste qu'un objet et, souvent, un objet en trop, embarrassant et qui fait souffrir. Dès lors, dans ce contexte, consentir à une recherche c'est un peu se poser en objet deux fois plutôt qu'une.

Qu'en est-il de la maladie? Si la langue française n'a qu'un mot pour désigner la maladie, la langue anglaise en possède trois, sans être synonymes : illness, disease et sickness. La maladie ne se présente pas de la même façon, si elle est vécue à travers le spectre de la maladie comme illness, disease ou sickness. Depuis la publication de l'ouvrage de Susser en 1973 (Susser, 1973), il est d'usage de faire une distinction de la manière suivante. Contrairement à la maladie comme disease qui se manifeste par des symptômes objectivables, la maladie comme illness, qui correspond à l'expérience vécue d'être malade, se caractérise par une souffrance, un malaise profond de ne pas appartenir au monde. De son côté, sickness renvoie à la maladie comme une catégorie sociale et culturelle.

En psychiatrie, les personnes aux prises avec un trouble mental se définissent souvent elles-mêmes comme étant des schizophrènes, des dépressifs. Il y a une autostigmatisation. Tandis que celles qui souffrent d'un trouble physique diront volontiers qu'elles souffrent de telle maladie. Par exemple, elles diront qu'elles souffrent d'un trouble cardiaque ou encore qu'elles ont attrapé telle maladie; «j'ai attrapé un cancer» ou «un rhume». Pour celles qui souffrent par exemple de schizophrénie, très peu diront qu'elles souffrent de schizophrénie. C'est toute la différence entre être et avoir. Comme si une maladie physique, malgré toutes les remises en question qu'elle peut entraîner, n'atteignait jamais ou peu l'identité de la personne, contrairement à une condition de santé mentale. Même si les professionnels de la santé tentent tant bien que mal de dire que les personnes sont aux prises avec une maladie mentale, pour celles qui vivent ces maladies, cela ne semble pas si évident. Pour certaines, il y a à peine eu un avant et un après la maladie. Alors, bien difficile de démarquer ce qui relève de la maladie et de l'identité. Dans ce contexte, il existe un lien très étroit entre la maladie et l'identité. Devenir le sujet d'une enquête sur la schizophrénie, c'est exposer son être au regard d’autrui.

En raison de cette étroite liaison entre la maladie et l'identité, sans que cela soit exclusif à la maladie mentale - pensons aux maladies chroniques - , pour le patient, raconter ce qu'il vit, c'est aussi se sentir réduit à une maladie, comme si on le vidait de son identité. Si tout ce que je vis se réduit à une maladie, alors il ne reste plus rien qui soit authentique et qui m'appartient vraiment en propre. La difficulté de trouver des sujets de recherche pour les chercheurs en santé mentale est probablement un bon signe : un signe de résistance devant l'objectivation. Il est d'usage de dire que la maladie mentale, c'est un peu l'enfer au moment de grandes crises aiguës. Mais, comme le souligne Sartre dans un autre contexte, l'enfer, ce sont les autres, qui m'épient. Alors, comment ne pas devenir méfiant? La méfiance ne serait-elle pas aussi paradoxalement le signe qu'il y a quelque chose chez l'individu qui résiste à sa destruction?

Il y a une autre expérience existentielle qui vient teinter le processus de consentement. Les patients vivent des expériences existentielles qui sont parfois difficilement partageables dans un langage commun et un langage scientifique. Ils ne se sentent pas reconnus dans les questions que les chercheurs leur posent. Les chercheurs, souvent, les obligent indirectement à mettre des mots sur ce qu'ils vivent, bien que ces mots ne soient pas toujours les leurs.

Chez les personnes souffrant de schizophrénie, les barrières entre le monde externe et interne sont souvent perméables. Il y a une perte de l'intimité. Ainsi, pour ces personnes, répondre à un questionnaire peut devenir une forme d'intrusion, une menace à l'intégrité et à l'identité, même si les chercheurs les assurent de garder la confidentialité. 
Plusieurs personnes atteintes de schizophrénie vivent une faible estime de soi avec une perte de confiance. De sorte que l'on se retrouve avec des personnes dont la méfiance est exacerbée. Elles éprouvent la peur d'être un cobaye, d'être abusées, d'être manipulées, et ce n'est pas l'histoire de la psychiatrie qui les contredira. L'histoire de la psychiatrie abonde de cas où, justement, les personnes furent utilisées comme des cobayes. Le procès de Nuremberg l'a mis en évidence. Encore récemment, on fait état dans les médias d'abus entourant la participation à la recherche en santé mentale sans le consentement des personnes : «Plus d'un millier de patients de la clinique de Marsens ont servi de cobayes à Sandoz» (RTS, 2017); «Gironde : un psychiatre condamné pour avoir pris des patients pour cobayes» (AFP, 2014); «Psychiatrie : traitements barbares et destruction du cerveau» $(\mathrm{CCDH}, 2017)$.

Elles peuvent devenir réticentes à l'idée de répondre à un questionnaire écrit. Cela peut les confronter à leur limite cognitive et à leur manque de concentration. Elles se sentiront incompétentes.

Finalement, la seule idée de signer un formulaire de consentement peut représenter une énorme barrière. Certaines personnes souffrant d'un trouble mental seront consentantes à participer à une recherche en santé mentale si elles ne sont pas dans l'obligation de signer un formulaire de consentement. Souvent, un consentement verbal est suffisant. Par contre, l'abstention ou le manque d'intérêt ne sont pas des formes de consentement (Kleinig, 2010) ni de refus.

Évidemment, pour certaines personnes, c'est tout l'opposé. Elles seront heureuses de participer à une recherche de type qualitatif. Cela leur donnera l'occasion d'objectiver ce qu'elles vivent, de prendre une distance. D'autres y voient l'occasion de parler de ce qu'elles vivent autrement que dans le contexte clinique où elles sont évaluées. Elles peuvent parler sans être jugées, c'est-à-dire sans avoir l'impression que tout ce qui sera dit sera ramené à la maladie (Capdevielle et coll., 1998). Chez d'autres naîtra l'impression de participer à une mission, de participer à l'avancement des connaissances, de se rendre utiles. Finalement, d'autres auront l'impression de sortir de leur isolement. Bref, participer à une recherche devient une opportunité de donner un sens à leur existence.

\section{Quand consentir ne veut rien dire ou presque}

Ce qui ressort de ce regard phénoménologique, c'est que le consentement ne repose pas strictement sur le traitement d'information. Il repose en grande partie sur le sens que les personnes accordent à la recherche. Cependant, il importe aussi de mieux comprendre la dimension logique de nos arguments entourant le consentement.

Par exemple, si je fais une réservation dans un hôtel, je m’attends à ce que certains services soient offerts. Mais il arrive que nous ayons de mauvaises surprises : par exemple, à proximité de l'hôtel, des travailleurs sont peut-être en train de construire un autre bâtiment, ce qui entraîne de forts bruits (en raison de l'utilisation de marteau-piqueur). Bref, on n'avait pas consenti à cela. Donc, si je consens à A, je ne consens pas nécessairement à $\mathrm{B}$. Je peux consentir à une relation sexuelle, mais pas à la transmission du VIH. Consentir ne veut pas dire consentir à tout. Ce n'est pas une carte blanche ou un chèque en blanc. On consent à telle et telle chose, mais pas à ceci ni à cela.

Un autre exemple. En consultation externe, nous avons exposé à un patient le projet d'une recherche à laquelle il a accepté de participer. Quelques jours plus tard, en début de matinée, le chercheur se présente à son domicile pour commencer une entrevue. Le patient refuse. Celui-ci venait de réaliser ce que cela impliquait 
pour lui de recevoir un étranger à son domicile. C'était pour lui une intrusion dans son intimité, dans son être. Cet exemple permet de distinguer deux registres différents qui ont été sollicités à deux moments différents. La première rencontre a fait appel à la dimension cognitive chez la personne, tandis que la deuxième rencontre a mis en évidence la dimension de l'expérience vécue. En premier lieu, c'était son intellect qui parlait et, en second lieu, ce fut son cœur, c'est-à-dire sa dimension affective. Inutile de dire que les chercheurs se situent généralement du côté de la raison et que les formulaires de consentement se concentrent sur la transmission d'information factuelle. Par conséquent, ils peuvent comprendre difficilement un refus, même si d'emblée ils acceptent cette éventualité, surtout que les personnes peuvent se retirer en tout temps d'une participation à une recherche sans fournir d'explication. Comme le mentionne Pascal (2000, p. 679), «le cœur a ses raisons que la raison ne connaît point». Même si l'on parle de la même chose, on comprend différemment. Cet exemple démontre que, même si le patient fut informé de tous les aspects entourant la recherche - la raison d'être de l'étude, la méthode utilisée, l'identification des chercheurs, les sources financières s'il y a lieu, la durée et la nature de la participation, les bienfaits anticipés, la confidentialité, etc. - il n’y a aucune exploration de son point de vue selon son vécu et ses motifs pour une éventuelle participation.

Cet exemple illustre aussi un autre aspect du consentement. La personne consent à la finalité de la recherche (l'idéal de l'avancement des connaissances), mais pas au moyen utilisé (une visite à domicile). Il existe une distinction entre la finalité et les moyens. On peut souhaiter obtenir un système de santé plus efficace, sans endosser les moyens utilisés (la perte d'emploi). La finalité ne justifie pas toujours et nécessairement les moyens. À cet égard, il existe deux positions : une position idéaliste qui se concentre sur la finalité et une position pragmatique qui se concentre sur les moyens. Pour la position idéaliste, la finalité (l'avancement des connaissances) justifie les moyens utilisés. Quant à la position pragmatique, ce sont les moyens qui déterminent la finalité.

Fréquemment, sur les formulaires de consentement, on avance l'idée que la personne qui participe à la recherche contribue à l'avancement des connaissances en vue de l'amélioration de la qualité de vie (Bacon, 1991). Certes, le progrès des savoirs constitue bien la visée de la recherche scientifique. Mais qu'en est-il dans les faits? Ce ne sont pas toutes les recherches qui font l'objet d'une publication et celles qui sont publiées ne sont pas toutes lues. De plus, le champ d'intérêt des chercheurs change constamment, autant à l'intérieur d'une carrière, selon les intérêts et les subventions, qu'entre les générations de chercheurs. Il n'y a pas nécessairement de continuité et d'accumulation de connaissances. Les changements de pratique et les changements sociaux ne reposent pas nécessairement sur l'avancement des connaissances. Parfois, les enjeux politiques et économiques prévalent (Doucet, 2002). Sommes-nous devant un mensonge, une manipulation? Est-ce que cette information est transmise à la personne?

\section{La compétence à l'aptitude}

Bizarrement, lorsque les personnes refusent ou changent d'idée, rapidement les chercheurs, comme les cliniciens, soupçonnent une inaptitude à consentir. Or, il faut le préciser, la maladie mentale ne signifie pas une inaptitude à réfléchir, à délibérer et à consentir. La personne peut très bien comprendre les informations, peser les avantages, les inconvénients, les risques, les alternatives, en faire une appréciation et prendre une décision. Il convient d'accepter aussi que le changement d'idée n'est pas une caractéristique de l'inaptitude. Après tout, comme le dit l'adage, il n'y a que les fous qui ne changent pas d'idée. En somme, le diagnostic psychiatrique à lui seul n'interfère pas à la capacité de consentir. La capacité à la prise de décision éclairée doit être évaluée à la lumière de la situation selon les critères de détermination de l'inaptitude tels qu'établis par la législation de la Nouvelle-Écosse. 
Dans la maladie mentale, comme avec une maladie physique, la souffrance peut être aiguë. La souffrance rend la personne vulnérable. Celle-ci veut être soulagée à tout prix. Elle peut être prête à tout. Par exemple, elle peut dépenser des fortunes dans des soins douteux, au cas où cela fonctionnerait. Même si la personne comprend bien le protocole de recherche, les avantages et les inconvénients, il se pourrait qu'elle accepte pour d'autres raisons que celles évoquées dans le protocole : recevoir des soins ou bénéficier d'un nouveau traitement pensant qu'elle n'a rien à perdre et tout à gagner. Comme le mentionnent Appelbaum et ses collègues (1982, 1987, 2004, 2008), il y a souvent de fausses conceptions qui entourent la recherche chez les patients, sans compter chez les chercheurs eux-mêmes. Les patients peuvent consentir à une recherche croyant recevoir des soins privilégiés. Pourtant ce sont deux finalités contraires : celle d'offrir des soins et celle de produire des données valides.

Chez plusieurs personnes, la maladie mentale se vit dans la souffrance. Elles vivent la maladie mentale comme une torture. Pourtant, sous la torture, on reconnaît la non-responsabilité. Avouer un crime sous la torture ou sous la menace n'est pas un aveu sur le plan juridique. En raison de la souffrance, il y a inaptitude, selon Kleinig (2010). Doit-on étendre ce raisonnement à la souffrance inhérente à la maladie? Dans ce cas, si l'on devait poursuivre le raisonnement, il y aurait très peu de recherches en santé mentale.

\section{Le volontarisme ou la coercition}

Nous avons vu que le consentement éclairé n'est pas aussi évident qu'on le croit. Il en va de même avec le consentement libre. Les chercheurs acceptent d'emblée qu'il ne doive pas y avoir de coercition. Cependant, nous pouvons nous demander si exercer une influence est une forme de coercition. II semble que non. Estce que persuader est une forme de coercition? Il semble que non. Remettre de l'argent pour dédommager, est-ce une forme qui compromet le libre consentement? Encore une fois, il semble que non, si le montant remis n'outrepasse pas les coûts générés. Pourtant, en santé mentale, les personnes sont très vulnérables à l'influence, à la persuasion et à recevoir d'autres sources de revenus. De plus, elles voudront consentir pour plaire ou pour ne pas déplaire. Elles consentiront pour ne pas être ostracisées ou stigmatisées. Elles aussi sont mues par le souci de maintenir l'alliance.

\section{Consentement et dialogue}

Le consentement ne peut pas se résumer à un simple acte d'intentionnalité, de cognition, de volonté et d'émotion. Les gens décident selon ce qui a du sens pour eux. Je ne consens pas seulement à des gestes, à des choses, mais surtout aux sens que ces gestes et ces choses représentent. Le consentement ne signifie pas nécessairement que je transferts mes droits à une autre personne, que je perds une partie de ma liberté. Le consentement doit être vu comme ce qui lie les personnes à la coopération. Consentir devient un acte de communication. Il devient préférable de concevoir le consentement comme un processus qui permet l'expression d'un choix libre et authentique (Roberts, 2002). Pourtant, dans un contexte de recherche, il existe une inégalité entre le chercheur et le participant. Le dialogue permet d'établir une égalité de parole pour pallier l'asymétrie des rôles.

Trouver la solution dans l'intersubjectivité, cela signifie créer un monde commun, un monde de partage, car ce monde commun n'est pas donné à l'avance, mais s'établit dans le dialogue. Mais de quoi parle-t-on exactement? De tout ce dont les personnes désirent parler? Principalement, on parle des motifs qui guident et justifient leur choix d'y participer, de refuser ou de se retirer. C'est le dialogue autour des motifs qui nous rapproche 
le plus d'un consentement éclairé. Comme le souligne Marzano, qui s'appuie sur le travail d'Anscombe, «pour pouvoir qualifier une action de volontaire et de libre, il faut qu'elle soit, au moins en partie, intentionnelle et qu'un individu puisse donner des raisons pour l'expliquer» (2006: 13). Par contre, cela exige un minimum de coercition : celle de penser ensemble les motifs. Est-ce que cela implique que les chercheurs doivent refuser une personne qui désire participer à une recherche s'ils considèrent que les motifs exprimés ne sont pas assez nobles? Doivent-ils colliger sur le formulaire les motifs du consentement? Est-ce une procédure trop lourde autant pour les chercheurs que pour les participants?

Nous avons vu qu'il y a un monde de différences entre ce qui relève de la cognition et ce qui relève du vécu. C'est pourquoi les chercheurs ne peuvent pas demander aux personnes susceptibles de participer à une recherche de consentir à ce qu'elles n'ont pas encore vécu. D'où l'idée que le consentement est un processus qui se déploie sur une échelle de temps. Avant, pendant et après la recherche, si celle-ci se déploie sur plusieurs étapes. Cela signifie qu'on n'a jamais fini de discuter des motifs entourant le consentement.

\section{Indications pragmatiques}

Chez plusieurs personnes souffrant d'un trouble psychiatrique, il n'est pas rare que nous retrouvions un manque de concentration. Souvent, après 10 minutes, on assiste à une baisse significative de la concentration. Cela implique qu'il devient avantageux de séquencer le processus de consentement et le déroulement de la recherche dans une même plage horaire. Par exemple, faire une courte intervention, suivie d'un repos de 15 minutes, par exemple pour fumer, suivi d'une autre courte intervention, suivie d'une autre période de repos de 15 minutes (biscuit et jus), ainsi de suite. Durant les pauses, il peut être approprié de ne pas les laisser seules.

Il convient aussi pour le chercheur de savoir que, si la personne ne se présente pas à la rencontre, cela ne signifie pas un refus. Il y a fréquemment des symptômes d'aboulie chez les schizophrènes. C'est l'un des critères du DSM-5 (APA, 2013), la référence la plus utilisée pour poser un diagnostic d’un trouble mental.

Nous savons qu'il est difficile pour plusieurs personnes de se déplacer en consultation externe, d'honorer leur rencontre planifiée avec leur professionnel de la santé, de sorte que ces personnes finissent par ne pas recevoir de soins. Pour cette raison, durant les années 1970, Stein et Test $(1976,1980)$ ont développé le suivi intensif dans la communauté afin de mieux répondre aux besoins de ces personnes. Je crois que les chercheurs devraient s'inspirer de ce service qui consisterait pour les professionnels de la santé à se déplacer, si possible, à domicile pour effectuer leur recherche.

\section{Conclusion}

Nous avons vu que l'idée du consentement libre et éclairé est un concept juridique qui demeure sur le plan éthique et philosophique controversé (Veatch, 2006). Par exemple, pour Spinoza (1954), la liberté se présente comme une illusion. Mais, pour des raisons pragmatiques, il est sûrement préférable de continuer ainsi.

Dans un contexte de santé mentale, nous croyons qu'une attitude trop rigide est contre-productive. En ce sens, il faut rétablir la tradition du bon sens ou du sens commun, même si, en elle-même, elle n'est pas suffisante pour assurer une sécurité aux patients, d'autant plus que nous pouvons toujours nous interroger sur qui détient la vérité du bon sens ou du sens commun. De plus, le sens commun n'est pas un critère, surtout que le sens commun, principalement en psychiatrie, n'est pas toujours la chose la mieux partagée. Il est souvent inexistant. C'est la même chose pour nous. C'est pour cela qu'on se donne des critères, même si ceux-ci n'épuisent 
pas toute la question du consentement. Cela devient, selon Aristote, une question de sagacité (phronesis), c'est-à-dire de jugement en situation qui interpelle la prudence en recherchant un équilibre à travers la mise en œuvre d'un dialogue entre les aspects plus subjectifs et ceux plus objectifs, entre divers désirs et diverses règles. II s'agit de parvenir à un équilibre entre la protection des personnes aux prises avec un trouble mental et le besoin d'accroître nos connaissances, d'améliorer notre condition de vie et de produire de meilleurs outils d'intervention pharmacothérapeutiques, psychothérapeutiques et d'intégration sociale. Cette recherche de sagacité ne vise pas qu'à se protéger d'une éventuelle poursuite judiciaire, mais aussi bien à développer chez le chercheur une attitude éthique plus responsable, suivant laquelle la recherche scientifique n'est pas une fin en soi (Christopoulos et coll., 2008).

Même si les chercheurs, en s'inspirant d'Hippocrate, sont portés par de bonnes intentions à agir en faveur de l'intérêt supérieur des participants à la recherche et que notre société s'est donné de grandes valeurs pour nous guider dans la protection des personnes, l'histoire de la recherche en santé mentale démontre à souhait que cela n'est pas suffisant. C'est la raison pour laquelle un dialogue, qui ne consiste pas qu'à effectuer un traitement de l'information, est encore le meilleur outil pour mettre en lumière le sens d'une participation à la recherche. Si le consentement repose sur l'autonomie, il faudrait penser l'autonomie, non pas comme une autodétermination, mais comme la capacité de parler en son propre nom de ce qui fait sens. Être autonome, c'est consentir à travailler à sa propre vérité. Cela est très contraignant et rejoint la conception kantienne de l'autonomie qui s'oppose à une conception de l'autonomie qui consiste à agir selon ses préférences et son bon plaisir (Kant, 1943). Être autonome, c'est oser penser par soi-même et comprendre que le concept de consentement s'inscrit dans une histoire culturelle et juridique. II s'agit d'en saisir les sens possibles avec ses avantages, sans se laisser écraser par son évidence, afin d'éviter qu'elle se présente comme une hétéronomie. Dès lors, il ne s'agit pas seulement de respecter l'autonomie d'autrui, mais d'en prendre soin (Benito et Garcia, 2016).

Si, pour des raisons pragmatiques, il est justifié de simplifier la complexité du vécu, il en va autrement sur le plan de la pensée, car nous risquons de nous «éloigner à la fois de la réalité et de l'éthique» (Marzano : 12). C'est le travail de la pensée qui peut nous protéger de toute forme de totalitarisme. Comme le mentionne Doucet, «les décisions humaines ne sont jamais prises dans l'idéal, elles sont négociées dans les limites qu'impose la vie» (2002 : 30). Cependant, comme le mentionne à son tour Kant (1986), l'idéal ne doit pas nous empêcher d'y penser, sinon on risque d'éliminer l'éthique de la recherche. Si, pour Rabelais (1964 : 139), «la science sans conscience est ruine de l'âme», il faudrait préciser que la science sans un examen de nos présupposés et de nos motifs ne peut que nous nuire, ce qui va à l'encontre de l'un des principaux principes si chers à Hippocrate (1994 : 367), primum non nocere. Pour cela, on ne peut pas compter sur la science, car celle-ci (non pas les chercheurs), comme le souligne Heidegger (1958), ne pense pas, étant donné que, par principe méthodologique, elle a évacué de son questionnement la question du sens.

Il existe plusieurs raisons qui justifient le consentement. Sauf que les raisons ne se trouvent pas toutes dans le réel, mais dans les idées qui organisent notre expérience du consentement et qui se développent dans l'espace de l'intersubjectivité, et qui n'apparaissent qu'à la conscience. Il ne s'agit pas d'accroître nos connaissances, mais surtout d'élargir le spectre de notre compréhension, car le consentement n'est pas qu'un problème à résoudre, mais quelque chose qui donne à penser. 


\section{Bibliographie}

Association médicale mondiale (AMM). (2013). Déclaration d'Helsinki de l'AMM-Principes éthiques applicables à la recherche médicale impliquant des êtres humains, Fortaleza.

AFP. (2014, June 5). Gironde: un psychiatre condamné après avoir pris des patients pour cobayes. Midi libre. Repéré à http://www.midilibre.fr/2014/06/05/gironde-un-psychiatre-condamne-pour-avoir-pris-despatients-pour-cobayes,1002281.php.

Ambroselli, C. (1988). L'Éthique médicale, Paris : Presses Universitaires de France.

American Psychiatric Association (APA). (2013). DSM-5 (Manuel diagnostique et statistique des troubles mentaux)

Appelbaum, P., Roth, L.H. et Lidz, C. (1982). «The therapeutic misconception: Informed consent in psychiatric research », International Journal of Law and Psychiatry, 5 (3-4), 319-329.

Appelbaum, P., Roth, L.H., Lidz, C., Benson, P. et Winslade, W. (1987). False Hopes and best data: Consent to research and the therapeutic misconception », The Hastings Center report, 17 (2), 20-24.

Appelbaum, P. (2000). Decisional capacity for informed consent in schizophrenia research, Archive of General Psychiatry, 57 (6), 533-538.

Appelbaum, P., Lidz, C., Grisso, T. (2004). Therapeutic misconception in clinical research: frequency and risk factors, Psychiatry Publications and Presentations, 26 (2), 1-8.

Appelbaum, P., Lidz, C. (2008). Twenty-five years of therapeutic misconception, The Hastings Centre report, 38 (2), 5-6.

Aristote. (1994). Éthique à Nicomaque, Paris: Vrin.

Bacon, F. (1991). Du progrès et de la promotion des savoirs, Paris, Gallimard.

Beecher, H. K. (1966). Ethics and Clinical Research, The New England Journal of Medicine, 274, 1354-1360.

Benito, J.J. et Garcia, S.E.R. (2016). Informed consent in the ethics of responsibility as stated by Emmanuel Levinas. Medicine, Health Care and Philosophy, 19 (3), 443-453.

Bernard, C. (2008). Introduction à l'étude de la médecine expérimentale, Paris : Flammarion.

Bouaziz, N., Boussion, S., Benadhira, R., Braha, S., Wojakiewicz, A. et Januel, D. (2011). L'éthique de la recherché en psychiatrie adulte, Annales medico-psychologiques, 169 (6), 367-372.

Capdevielle, V., Raffard, S., Bayard, S., Garcia, F., Baciu, O., Bouzigues, I., Boulenger, J.P. (2009). Competence to consent and insight in schizophrenia: is there an association? A pilot study. Schizophrenia Research, 108, 272-279.

Capdevielle, V, Laterrasse, C. et Doucet, C. (1998). Position de chercheur et souffrance du sujet, Psychologie Clinique, 5, 89-117.

Christopoulos, P., Trompoukis, K. et Gourzis, P. (2008). Ethical aspect of psychiatric research: patient's capacity to provide informed consent, Annals of General Psychiatry, 7 (Suppl I), S244. DOI : 10.1186/1744-859X-7S1-S244

Code civil du Québec, RLRQ, c. C.c.Q.-1991. 
Commission des citoyens pour les Droits de l'Homme (CCDH). (2017, December 5). Psychiatrie: traitements barbares et destruction du cerveau. Repéré à https://www.ccdh.fr/Psychiatrie\%C2\%A0-traitements-barbares-et-destruction-du-cerveau_a300.html

Instituts de recherche en santé du Canada, Conseil de recherches en sciences naturelles et en génie du Canada, Conseil de recherches en sciences humaines du Canada. (1998, avec les modifications de 2000, 2002 et 2005). Énoncé de politique des trois Conseils : Éthique de la recherche avec des êtres humains. (cité dans le texte comme EPTC). http://www.ger.ethique.gc.ca/archives/tcps-eptc/docs/TCPS\%20octobre\%202005_F.pdf

Doucet, H. (2002). L'éthique de la recherche. Guide pour le chercheur en sciences de la santé, Montréal, PUM.

Faden, R.R. et Beauchamp, T.L. (1986). A History and Theory of Informed Consent, New York: Oxford University Press.

Heidegger, M. (1958). Essais et conferences, Paris: Gallimard.

Hippocrate. (1994). De l’Art medical, Paris: Le Livre de poche.

Kant, E. (1943). Critique de la raison pratique, Paris : Presses Universitaires de France.

Kant, E. (1986). Euvres philosophiques, tome III, Paris : Gallimard.

Kleinig, J. (2010). The nature of consent. Dans Miller, F.G. et Wertheimer, A. (dir.). The Ethics of Consent, New York: Oxford University Press.

Kovnick, J.A, Appelbaum, P.S., Hoge, S.K. et Leadbetter, R.A. (2003). Competence to consent to research among long-stay inpatients with chronic schizophrenia, Psychiatric Services, 54 (9), 1247-1252.

Lehrman, N.S. et Sharav, V.H. (1997). Ethical Problems in Psychiatric Research, The Journal of Mental Health Administration, 24 (2), 227-250.

Marzano, M. (2006). Je consens, donc je suis..., Paris : Presses Universitaires de France.

Pascal, B. (2000). Euvres complètes, tome II, Paris : Gallimard.

Rabelais (1964). Pantagruel. Paris : Le livre de poche.

Ratcliffe, M. (2015). Experiences of Depression: A Study in Phenomenology, Oxford : Oxford University Press.

Ringuet, J.N. (2010). «L'érosion du consentement en recherche sur des êtres humains au Québec». Éthique publique, 12 (1), 31-53.

Roberts, L.W., Solomon, Z., Roberts, B.B. et Keith, S.J. (1998). Ethics in Psychiatric Research. Resources for Faculty Development and Resident Education, Academic Psychiatry, 22 (1), 1-20.

Roberts, L.W. (2002). Ethics and mental illness research. The Psychiatric Clinics of North America, 25 (3), 525 545.

Rhodes, R. (2005). Rethinking research ethics, The American Journal of Bioethics, 5 (1), 7-28.

RTS Info. (2017, 27 janvier). Plus d'un millier de patients de la clinique de Marsens ont servi de cobayes à Sandoz. Repéré à https://www.rts.ch/info/suisse/8342842-plus-d-un-millier-de-patients-de-la-clinique-de-marsens-ont-servi-de-cobayes-a-sandoz.html

Sartre, J.-P. (1943). L'Être et le néant. Paris: Gallimard.

Spinoza. (1954). Oeuvres complètes, Paris: Gallimard. 
Stanghellini, G. (2004). Disembodied spirits and deanimated bodies. The Psychopathology of common sense, Oxford: Oxford University Press,

Stein et Test (1976) Retraining Hospital Staff for Work in a Community Program in Wisconsin, Hospital Community Psychiatry, 27 (4), 266-268.

Stein, L.I. et Test, M.A. (1980). An alternative to mental health treatment. 1: Conceptual model, treatment program, and clinical evaluation », Archiv General Psychiatry, 37, 392-397.

Susser, M. (1973). Causal thinking in the health sciences, New York: Oxford University Press.

Test, M.A. et Stein, L.I. (1976). «Practice guidelines for the community treatment of markedly impaired patients». Community Mental Health, 12, 72-82.

Toombs, S.K. (1993). The Meaning of Illness. A Phenomenological Account of the Different Perspectives of Physician and Patient, Dordrecht: Kluwer Academic Publishers.

Veatch, R. (2006). «How Philosophy of Medicine Has Changed Medical Ethics », Journal of Medicine and Philosophy, 31 (6), 585-600. 
TITRE: RECRUTER DES «TRAVAILLEURS DE L'OMBRE» : LE DÉFI DE LA PARTICIPATION CHEZ LES PRÉPOSÉS AUX BÉNÉFICIAIRES

Auteur(s): Émilie Allaire et Nathalie Jauvin

PublicATION: RECRUTEMENT ET CONSENTEMENT À LA RECHERCHE : RÉALITÉS ET DÉFIS ÉTHIQUES

PAGES: $86-97$

ISBN: 978-2-7622-0357-8

Directeurs: Ana Marin, BÉAtrice Eysermann et Michel T. GIROUX

URI: HTTP://HDL.HANDLE.NET/11143/14111

DOI: HTTPS://DOI.ORG/10.17118/11143/14111 


\section{Recruter des «travailleurs de l'ombre» : le défi de la participation chez les préposés aux bénéficiaires}

Émilie Allaire, M.A., professionnelle de recherche, CIUSSS de la Capitale-Nationale

Nathalie Jauvin, Ph. D., Centre d'excellence sur le vieillissement de Québec, CIUSSS de la Capitale-Nationale

Résumé : Les préposés aux bénéficiaires forment le groupe de travailleurs le plus important, en termes de nombre, du réseau de la santé et des services sociaux québécois. Ils travaillent pourtant dans l'ombre des autres groupes professionnels, et leur implication au sein de l'organisation est souvent minimale. Ainsi, lorsque nous les sollicitons pour participer à des activités de recherche qui les concernent, très peu répondent à l'appel. Ce texte présente, à partir d'analyses d'entretiens réalisés dans ce milieu, certains des défis et des pistes qui permettraient de faciliter le recrutement des préposés aux activités de recherche. Parmi les défis importants à relever par les chercheurs, il y a tout particulièrement celui de gagner la confiance de ce groupe de travailleurs. II nous apparaît aussi essentiel de dégager des moyens pour rapprocher l'univers de la recherche des CHSLD et de ceux qui y travaillent afin de les faire participer pleinement au développement des connaissances qui les concernent.

Mots-clés : préposés aux bénéficiaires, centres d’hébergement, participation, recrutement, éthique 


\section{Introduction}

Nous menons depuis plusieurs années des recherches qualitatives portant sur la santé psychologique au travail des préposés aux bénéficiaires (PaB) œuvrant au sein des centres d'hébergement de soins de longue durée (CHSLD) québécois. Ces recherches, d'abord exploratoires, devraient sous peu, à l'initiative du milieu, prendre la forme de recherches interventionnelles afin d'accompagner des centres d'hébergement dans la mise en place de processus préventifs visant une amélioration du bien-être de ces préposés. Nos travaux ont été, dès le départ, développés via une approche participative ${ }^{1}$ dans le cadre de laquelle nous collaborons étroitement avec nos partenaires du milieu - représentants de la gestion et des travailleurs -, et ce, pour chacune des étapes de la recherche. Or, malgré le recours à une approche participative interpellant activement le comité de pairs ${ }^{2}$ qui les représente, nous rencontrons systématiquement des problèmes de recrutement auprès des préposés aux bénéficiaires que nous sollicitons pour participer à nos recherches.

Ce texte se veut avant tout une réflexion autour de cet enjeu fondamental qu'est la participation des préposés aux activités de recherche qui leur sont destinées. Pour amorcer cette démarche réflexive, nous illustrerons d'abord comment le contexte plus global de non-participation qui teinte le collectif des préposés éclaire les difficultés que nous rencontrons lors de nos activités de recrutement. Pour ce faire, nous proposerons donc dans un premier temps aux lecteurs un bref tour d'horizon des enjeux actuels touchant, d'une part, les préposés aux bénéficiaires, mais aussi, plus largement, ceux qui concernent le milieu de travail des CHSLD. Nous traiterons ensuite des défis plus spécifiques rencontrés dans le cadre de nos activités de recherche menées auprès des préposés, puis nous présenterons différents enjeux éthiques et pratiques soulevés par le processus de recrutement afin d'illustrer les enjeux potentiels liés à l'implantation du projet interventionnel en préparation $^{3}$. Enfin, nous analyserons succinctement les méthodes de recrutement auxquelles nous avons eu recours jusqu'ici pour finalement dégager certaines pistes de solution qui pourraient contribuer à influencer l'adoption de stratégies de recrutement plus adaptées à cette population de travailleurs.

\section{Le métier de préposé aux bénéficiaires : quelques éléments de contexte}

Les préposés aux bénéficiaires ${ }^{4}$, selon l'appellation québécoise, et aides-soignantes, selon la dénomination française et belge, sont des travailleurs qui prodiguent des soins d'assistance dans les milieux hospitaliers. Ils forment d'ailleurs le groupe de travailleurs le plus nombreux dans le réseau de la santé et des services sociaux au Québec. Au sein des CHSLD, ils soutiennent de façon continue les personnes hébergées en offrant quotidiennement des soins d'hygiène et en s'assurant de l'alimentation, de l'habillement, des déplacements et de la mobilisation de chaque résident (Aubry et Couturier, 2014a). Ces tâches d'assistance, même si elles

1. L'approche participative implique la participation des acteurs concernés par la démarche de recherche à l'ensemble du processus: l'identification des questions ou des thèmes, la préparation des outils de collecte de données, la planification de la stratégie de recrutement et de celle de la cueillette donnée, la participation active à des comités de suivi, l'élaboration d'activités de transfert et d'appropriation des connaissances (Côté et Jauvin, 2014). Nos partenaires principaux sont, dans le cadre de ces activités de recherche, les préposés aux bénéficiaires et leurs représentants (comité de pairs) ainsi que les représentants de la gestion. Dans notre cas, nos partenaires et les préposés, via le comité de pairs, s’impliquent à toutes les étapes de la recherche.

2. Comité formé de représentants des préposés qui vise principalement à transmettre de l'information aux pairs et à travailler collectivement dans le but d'améliorer leurs conditions de travail.

3. Nous débuterons sous peu un projet d'intervention préventive pour les préposés aux bénéficiaires en CHSLD. Cette intervention s'inscrit en continuité avec les travaux participatifs amorcés en 2011.

4. Le masculin sera utilisé tout au long du texte afin de faciliter la lecture. 
ne bénéficient que de peu de visibilité en raison de leur non-spécialisation, sont essentielles pour assurer le bien-être et la qualité de vie des personnes hébergées.

Plusieurs auteurs qualifient le métier de préposé de «sale boulot» : un travail ingrat, non spécialisé et caractérisé par des tâches très physiques qui sont prodiguées de manière directe sur le corps des personnes (Arborio, 1995; Arborio, 2012; Lhuilier, 2005). Ces tâches peu enviables maintiennent d'ailleurs les préposés en bas de la hiérarchie hospitalière (Dubois, 2014; Marché Paillé, 2011). Très peu de prestige est en effet accordé à ce métier, même si, dans les sociétés contemporaines, on voue généralement un grand respect aux travailleurs de la santé ${ }^{5}$ (Dubois, 2014; Marché Paillé, 2011). Qu'on pense notamment au prendre soin des infirmières et au pouvoir de guérison des médecins, qui constituent des exemples de compétences médicales honorables, couramment encensées. L'honorabilité des métiers en milieu hospitalier apparaît, dans les faits, globalement établie en fonction d'une hiérarchisation des tâches liée à la formation académique reçue. Les préposés, qui effectuent des tâches non spécialisées pour lesquelles l'obtention du diplôme demande peu de temps, se retrouvent ainsi plutôt en marge, dans une position organisationnelle qui fait en sorte qu'on ne les sollicite que rarement pour entendre leur point de vue au sein des diverses structures de l'organisation.

Réduire ce métier au concept du sale boulot est cependant fort limitatif, et il importe de souligner l'importance du savoir-faire acquis sur le terrain par ces travailleurs. À travers ces tâches non spécialisées, les préposés pratiquent sans contredit un travail fondamental de care, c'est-à-dire un travail qui renvoie aux soins, à l'humain, à la globalité et à la relation sociale (Arborio, 2012; Aubry et Couturier, 2014a; Benelli et Modak, 2010; Saillant et Gagnon, 1999). Ils sont en effet détenteurs de savoirs profanes indispensables aux soins qu'ils ont à offrir, des savoirs qui se rattachent non pas à la science, mais, plutôt, aux compétences développées dans l'action, à leur manière d'être et d'agir avec les résidents (savoir écouter, savoir toucher en douceur, savoir entrer en relation et savoir s'adapter à chaque patient) (Arborio, 1996; Aubry et Couturier, 2014a). Bien qu'essentielles à leur travail auprès des résidents, ces connaissances non spécialisées ne sont cependant pas formellement reconnues par les autres professionnels de leurs institutions (Bowers, Esmond, et Jacobson, 2003; Lhuilier, 2005; Pfefferle et Weinberg, 2008). Les préposés travaillent donc essentiellement dans l'ombre des autres groupes professionnels et leur travail est le plus souvent invisible, ce qui contribuerait à réduire leur implication au sein de l'organisation.

Les préposés aux bénéficiaires vivent, en fait, une double invisibilité (Bonnet, 2006). D’une part, comme nous venons de le voir, on ne leur accorde que très peu d'espace au sein même de leur organisation. On les invite d'ailleurs rarement à prendre part aux décisions institutionnelles, aux réunions multidisciplinaires ou aux activités académiques ou de recherche qui ont cours dans leur milieu (Allaire, Gagnon, et Jauvin, 2015; Allaire, Jauvin, et Duchesne, 2015). D’autre part, ces mêmes préposés effectuent un travail très peu reconnu socialement, dont on ne parle essentiellement qu'en période de couverture médiatique négative, lorsqu'un incident est rapporté par les médias. Ils occupent alors la première place sous les projecteurs, mais pour des motifs peu élogieux et qui ne participent en rien à valoriser leur profession. Cette invisibilité sociale de ce groupe de travailleurs se manifeste aussi, soulignons-le, par le peu de regards scientifiques qui se sont effectivement posés sur ce métier; alors que d'autres professions du secteur de la santé ont été beaucoup plus largement étudiées, qu'on pense aux médecins et aux infirmières par exemple.

5. Les préposés aux bénéficiaires sont considérés par l'état québécois comme étant « des travailleurs du réseau de la santé qui sont intégrés aux équipes de soins pour aider le personnel infirmier et hospitalier ainsi que les médecins dans la prestation de base des patients » (Ministère du Travail, de l'Emploi et de la Solidarité sociale, site web). 


\section{Travailler dans un milieu en constante transformation}

En plus de pratiquer un métier méconnu, les préposés aux bénéficiaires travaillent au sein de CHSLD qui ont été exposés à des transformations majeures au cours des vingt dernières années. Ces changements, touchant à la fois les caractéristiques des personnes hébergées et les dimensions structurelles des établissements, affectent négativement leur travail (Allaire, Jauvin, et Duchesne, 2015; Aubry et Couturier, 2014a; Bélanger, 1998). Avec le prolongement de la vie à domicile, les personnes hébergées, de plus en plus âgées, arrivent en centre d'hébergement affectées d'une perte d'autonomie plus grande, le plus souvent attribuable à des problèmes cognitifs ou de santé mentale. Les soins à prodiguer à ces résidents demandent donc de plus en plus de temps. De plus, les préposés interviennent dorénavant beaucoup plus fréquemment auprès de résidents manifestant des problèmes comportementaux, ce qui complexifie considérablement leur pratique de soin. Et ce, dans un contexte caractérisé par le manque de formation et d'outils de prévention pour faire face à ces situations (Aubry et Couturier, 2014b).

À cela s'ajoute un milieu organisationnel marqué par plusieurs réformes et compressions budgétaires successives ayant contribué à une augmentation de la charge de travail, à une accélération de la cadence et, incidemment, au manque de temps pour effectuer les tâches prescrites. À ces contraintes se superposent aussi certains paradoxes entourant l'application des soins qui accentuent les défis à relever pour les préposés aux bénéficiaires (Aubry, Couturier et Gilbert, 2014). L'approche milieu de vie en est un bon exemple : il s'agit d'une approche qui incite les préposés à effectuer des soins personnalisés (respect des habitudes de vie), à stimuler les capacités et les forces restantes des résidents ainsi qu'à les faire participer à leurs soins. Or, les réalités institutionnelles ne sont souvent pas propices à la mise en place de ces activités quotidiennes, ce qui accentue la pression sur les travailleurs qui ne bénéficient pas de ressources pour répondre adéquatement à de telles initiatives (Aubry, Couturier et Gilbert, 2014). Ce contexte organisationnel, marqué par une charge de travail élevée et un rythme de travail accéléré, génère inévitablement des atteintes à la santé physique et psychologique chez ces travailleurs, que ce soit en termes de stress, d'épuisement professionnel, de problèmes de sommeil, d’absentéisme, de roulement du personnel (Allaire, Gagnon et Jauvin, 2015; Bélanger, 1998). Les préposés constituent d'ailleurs le groupe de travailleurs pour lequel on rapporte le plus grand nombre d'incidents indemnisés dans le réseau de la santé et des services sociaux (Bédard et Bélanger, 2013).

\section{Faire de la recherche participative dans un contexte non favorable à la participation}

Les préposés aux bénéficiaires forment, nous venons de le constater, un groupe de travailleurs auquel on s'intéresse très peu. Pourtant ils sont, au quotidien, exposés à des situations de travail qui les fragilisent. C'est dans l'objectif de contribuer à mieux comprendre la situation de ces travailleurs puis d'identifier et d'implanter des mesures préventives que nous avons débutées, en 2011, des activités de recherche au sein de CHSLD de la grande région de Québec. Nous avons dès lors opté pour une approche participative de recherche, considérant qu'il s'agit d'une avenue fort prometteuse pouvant offrir aux préposés la possibilité de s'engager activement dans l'instauration des processus de changement qui les concernent principalement. Cette façon de faire pourrait leur permettre de figurer au premier rang des décisions interventionnelles, leur redonnant ainsi une visibilité au sein de l'équipe (Bailey et coll., 2013) et de l'institution qui les emploie. 
Malgré la mise en place de diverses activités de recherche participative appuyées par le milieu, nous rencontrons systématiquement un obstacle majeur : les préposés, pourtant en très grand nombre, répondent fort peu aux demandes de recrutement des projets de recherche, et ce, tant pour la réalisation d'entrevues individuelles que collectives. En effet, très peu de préposés posent spontanément leur candidature pour participer aux recherches que nous menons, ce qui menace à chaque fois de réduire la taille de l'échantillon prévu au protocole de recherche et, conséquemment, de limiter la portée des résultats escomptés. II nous est donc apparu nécessaire d'explorer plus en profondeur cette question du recrutement chez les préposés, dans la perspective où nous avons prévu, sous peu, l'implantation d'un projet de recherche interventionnelle dont le succès repose fondamentalement sur l'engagement et la participation des travailleurs au processus de transformation. Pourquoi, donc, les préposés sont-ils si peu enclins à répondre au recrutement de recherche et, par conséquent, à participer aux études que nous leur proposons? C'est ce que nous avons cherché à comprendre.

\section{Des résultats de recherche éclairants : la participation comme enjeu transversal}

En scrutant attentivement le contenu des entretiens individuels et de groupes menés jusqu'ici, nous avons constaté qu'un enjeu majeur transcende les analyses, celui de la participation des préposés aux bénéficiaires aux activités de leur organisation. Et ce constat ne peut qu'avoir un impact sur l'intérêt de ces travailleurs à s'investir dans des projets de recherche qui les concernent.

Plusieurs éléments contextuels peu favorables à la participation des préposés aux activités de recherche ont pu être dégagés des témoignages recueillis auprès de préposés et des personnes qui les encadrent. Nous avons ainsi constaté que, sur un plan plus structurel d'abord, l'organisation fortement hiérarchisée des CHSLD n'encourage pas les préposés à se tailler une place au sein de l'institution. Non seulement leur participation n'est que rarement sollicitée, mais, le plus souvent, on les maintient dans une position où ils sont conditionnés à demeurer discrets, voire silencieux. Mais le manque de participation s'opère aussi sur un plan plus individuel, où chaque préposé ne voit pas ou ne voit plus l'intérêt de prendre la parole, alors que, individuellement et même collectivement, ils sont portés par un sentiment acquis de méfiance qui fait obstacle à toute participation. Cette vision négative entourant le prendre place se manifeste aussi sur le plan collectif, où on observe un manque de solidarité entre pairs et un manque de volonté à participer aux réflexions qui ont cours dans l'établissement, et ce, même lorsque, le cas échéant, on accorde aux préposés le droit de parole, et ce, que ce soit dans le cadre d'activités initiées par la direction ou même par le syndicat, auquel par ailleurs les préposés participent globalement très peu.

Il s'avère donc essentiel de considérer cet enjeu central de la participation afin d'identifier les meilleures avenues pour favoriser la collaboration des préposés et, ultimement, leur permettre d'avoir accès à une expérience de recherche les autorisant, justement, à prendre cette place qui leur revient. 


\section{Un projet de recherche interventionnelle chez les préposés : enjeux pra- tiques et éthiques}

Depuis l'amorce des travaux de recherche que nous menons sur les préposés aux bénéficiaires, notre partenariat avec le milieu est porté par l'intérêt partagé de développer une intervention préventive participative qui permettrait d'agir favorablement sur le bien-être des préposés. Mais, avant d'amorcer cette étape cruciale de la recherche, il nous est apparu fondamental de réfléchir à des stratégies efficaces pour impliquer les préposés puisque, comme nous venons de le constater, les étapes antérieures de recherche nous ont, justement, dévoilé que la participation - pourtant fondamentale à une intervention participative - était loin d'être acquise. Or, développer une démarche de prévention exige une implication des travailleurs qui seraient notamment sollicités pour siéger sur différents comités responsables de développer et d’implanter l'intervention.

Devant les limites préalablement cernées et les défis relatifs à la participation anticipée, nous nous retrouvons, comme chercheurs, confrontés à des enjeux pratiques et éthiques importants sur lesquels il convient de se pencher avant d'amorcer l'intervention. D'une part, sur un plan pratique, une question centrale s'impose : L'implantation d'une démarche de prévention, dans des conditions de non-participation, est-elle une activité réaliste alors que le recrutement des préposés à de simples entrevues individuelles ou collectives constitue un défi particulièrement difficile depuis le début de nos activités de recherche? Et ce dans un contexte où les établissements de soins sont traversés par une vaste transformation organisationnelle susceptible de générer une pression additionnelle sur les préposés à participer ${ }^{6}$ ? D’autre part, nous sommes aussi invités à nous interroger sur les risques éthiques potentiels de faire la promotion d'une telle activité, qui risquerait, en l'absence de participation, de ne pas atteindre les objectifs poursuivis et, au final, de renforcer peut-être le sentiment d'invisibilité ou de méfiance et donc d'affecter négativement la situation de ces travailleurs. En d'autres mots, l'échec d'une telle démarche pourrait-il, ultimement, engendrer une situation plus lourde encore pour ces travailleurs? Mais, par ailleurs, est-il plus éthique de choisir de mettre fin aux activités de recherche auprès de ces travailleurs que l'on sait particulièrement fragilisés en raison de ces circonstances, sachant que nous cesserions des activités ayant un réel potentiel d'améliorer la situation?

\section{Le recrutement des préposés : défis et pistes}

En analysant attentivement ces enjeux, nous avons fait le choix, avec le milieu partenaire, de poursuivre les activités de recherche amorcées et de miser sur une meilleure compréhension des conditions propices à la participation à la recherche interventionnelle en nous penchant d'abord sur le processus de recrutement luimême afin de l'optimiser. Comment peut-on ainsi, à partir de l'état actuel de nos travaux, mieux comprendre la non-participation des préposés au recrutement de nos projets de recherche, quels sont les motifs qui se cachent derrière ces refus?

Nous avons eu au cours des dernières années l'occasion d'entendre, à de nombreuses occasions, les préposés et leurs supérieurs sur la question du recrutement ou de la participation aux activités de recherche. De ces témoignages ont émergé quelques éléments qui font globalement consensus et qui représentent, ultimement, des obstacles bien concrets à surmonter au cours des prochaines années :

6. La fusion du Centre de santé et services sociaux (CSSS) d'origine au nouveau Centre intégré universitaire en santé et services sociaux (CIUSSS) a engendré des transformations organisationnelles majeures, entre autres au sein des établissements où nous effectuions notre collecte de données. Dans un tel contexte de mouvement, nos activités de recherche ont été affectées, et les préposés, déjà difficiles à recruter en contexte régulier, ont été encore plus difficiles à rejoindre. 
1. les préposés ne connaissent pas du tout ou très peu l'univers de la recherche, ce qui les fait douter quant aux avantages de participer à une activité qui leur est proposée;

2. souvent épuisés par le temps qu'ils passent au CHSLD, ils sont très peu enclins à sacrifier du temps libre pour participer à des projets qui se déroulent en dehors de leurs heures de travail;

3. ils sont souvent méfiants et ils sont également préoccupés par des questions de confidentialité (c.à-d. dévoilement de leur participation ou encore des propos tenus lors de l'entretien);

4. ils n’ont généralement pas confiance lorsque les sollicitations pour participer à une recherche sont portées par des personnes en autorité?.

Comme on peut le constater, la question de la méfiance est particulièrement récurrente dans les discours des personnes que nous avons rencontrées. Parmi les défis importants à relever, il y a donc, certes, celui de gagner la confiance de ce groupe de travailleurs. Afin de mieux faire connaître nos activités, de déconstruire graduellement ces préjugés qu'entretiennent les préposés à l'égard de la recherche et de réduire peu à peu cette méfiance, nous collaborons étroitement, depuis quelques années déjà, avec la direction de l'établissement ainsi qu'avec le comité de pairs des préposés aux bénéficiaires. Ce comité, qui réunit des représentants des préposés, vise principalement à transmettre de l'information aux pairs et à travailler collectivement dans le but d'améliorer leurs conditions de travail. Nos stratégies de recherche, sur le terrain, sont donc toujours fortement arrimées avec ce comité. Ainsi, nos échanges avec les membres de ce comité permettent, d'une part, de valider si le projet est pertinent pour les préposés et, d'autre part, s’il est réaliste dans le cadre du contexte organisationnel actuel. À son tour, le comité agit comme courroie de transmission vers les préposés. II fait notamment circuler l'information sur les activités de recherche et il participe activement aux démarches de recrutement en publicisant le projet et en expliquant aux préposés qu'ils représentent les enjeux de la recherche.

Dans l'ordre, les stratégies de recrutement élaborées jusqu'ici avec le comité de pairs se résument ainsi :

1. Dans le processus d'élaboration d'un projet de recherche, nous allons tout d'abord discuter avec les membres du comité de pairs afin de vérifier leur intérêt pour le sujet et, le cas échéant, échanger sur les différents paramètres à considérer, notamment sur le processus de recrutement des participants.

2. Une fois le financement reçu et l'acceptation du comité d'éthique obtenue, nous rencontrons à nouveau le comité de pairs, cette fois-ci pour planifier en détail l'opération de recrutement et, notamment, discuter de l'élaboration de l'affiche qui servira à solliciter la participation des travailleurs.

3. Les membres du comité s'occupent ensuite de la distribution des affiches aux chefs d'unité qui, à leur tour, informent les préposés dans le cadre des réunions d'équipe.

4. Les membres du comité de pairs s'engagent aussi activement à faire connaître le projet, à apposer dans leur propre centre d'hébergement les affiches de recrutement et à susciter la participation de leurs collègues préposés.

Les étapes un et deux, qui touchent plus spécifiquement les rencontres avec le comité de pairs, fonctionnent généralement de façon très efficace : l'intérêt de la direction et du comité de pairs pour les recherches participatives que nous développons ensemble est bien présent. Les étapes trois et quatre soulèvent, quant à elles, quelques difficultés pour lesquelles il faudra, dans l'avenir, trouver des solutions afin de favoriser un processus qui suscitera une participation plus importante des préposés.

7. Or, en raison de la structure organisationnelle et du manque d'occasion de rejoindre les préposés, nos recherches sont souvent publicisées par les chefs d’unité, qui sont les supérieurs immédiats des préposés. 
Un premier défi concerne le dégagement des membres du comité des pairs pour s'engager plus activement dans le processus de recrutement afin de mieux faire connaître le projet. Jusqu'ici, sur le terrain, les représentants du comité de pairs disposent de peu de temps sur les lieux de travail pour transmettre l'information relative aux projets de recherche. Ils doivent donc compter davantage sur la collaboration des chefs d'unité afin de faire circuler l'information. Ainsi, dans un contexte où ils sont déjà fort chargés et qu'ils ne disposent pas des conditions pour rencontrer leurs collègues, cette tâche s'avère exigeante et certains ont même tendance à céder l'ensemble de la responsabilité du recrutement à leurs chefs d'unité, des personnes qui sont eux aussi, par ailleurs, exposées à un contexte marqué par une charge de travail très élevée qui ne leur laisse que peu de temps pour s'impliquer dans une démarche de recrutement. Puisque le temps de dégagement nécessaire pour procéder à un exercice de recrutement efficace semble être un facteur important, il faudrait vraisemblablement identifier des stratégies permettant d'améliorer les conditions de recrutement et, par le fait même, l'expérience des recruteurs.

Le second défi important concerne davantage l'identification des personnes sur qui devrait reposer le recrutement. Du côté des chercheurs, l'accès direct aux préposés est rendu particulièrement difficile en raison, notamment, du manque de modalités pour les rejoindre (c.-à-d. impossibilité de dégager du temps sur le temps de travail, absence d'adresse de courriel institutionnelle pour les préposés). Du côté des représentants syndicaux, la tâche apparaît également ardue puisque les préposés sont globalement assez peu réceptifs aux sollicitations de leur propre syndicat. Et, comme nos expériences de recherche nous l'ont démontré, la prise en charge du recrutement par les supérieurs suscite pour plusieurs une certaine méfiance et freine conséquemment bon nombre de préposés dans un contexte où certains souhaitent d'ailleurs que leur participation demeure confidentielle. Visiblement, le processus de recrutement le plus efficace jusqu'ici est celui pris en charge par les membres du comité de pairs. Puisque l'ouverture des préposés à s'engager dans un processus de recherche semble plus grande lorsque le projet est présenté par un pair, il faut donc voir, prioritairement, à bonifier le processus de recrutement et à identifier les conditions nécessaires pour que des représentants des pairs, qu'ils soient membres du comité de pairs ou non, puissent s'engager pleinement dans un tel processus.

\section{Rendre visible la recherche}

Il va sans dire que, pour favoriser la participation des préposés aux activités de recherche qui les concernent, il est nécessaire de poursuivre à long terme cette précieuse collaboration avec le comité de pairs des préposés aux bénéficiaires. Nos échanges aident, d'une part, à rapprocher le milieu de la recherche de ces travailleurs et, d'autre part, ils contribuent à réfléchir, tous ensemble, à la mise en place de stratégies de recherche afin, notamment, d'améliorer le recrutement et la participation des préposés.

II nous apparaît aussi essentiel, pour nos activités futures, de dégager des moyens pour rapprocher l'univers de la recherche des CHSLD et de rendre visibles les travaux de recherche qui concernent les préposés au sein de leur propre groupe, mais, aussi, au niveau de l'institution toute entière. La visibilité des projets de recherche dans les CHSLD ne peut pas reposer que sur les épaules des chefs d'unité qui sont eux aussi soumis à un fardeau de tâches important. Cette visibilité doit plutôt se construire en partenariat, via une collaboration impliquant le comité de pairs, les chercheurs et la direction des CHSLD. Cela suggère aussi que les équipes de recherche identifient des moyens pour se rapprocher du terrain, pour être plus présentes, visibles et accessibles dans les centres d'hébergement où il est possible de mener, par exemple, diverses activités de transfert de connaissances adaptées. Rendre visibles les projets de recherche et améliorer le recrutement permettrait par ailleurs non seulement de redonner une visibilité aux préposés aux bénéficiaires, mais contribuerait également à leur 
accorder un espace de parole et une marque de reconnaissance, ce qui serait à même de susciter, de manière positive et plus durable, la participation des préposés à l'organisation.

Nous terminons en suggérant quelques pistes de solution préliminaires qui permettraient, pour les travaux à venir, d'assurer de meilleures conditions de recrutement:

- $\quad$ Rapprocher la recherche du terrain, c'est-à-dire rendre la recherche et ses résultats visibles et accessibles afin de démystifier les objectifs poursuivis, les méthodes employées et d'optimiser les modalités de transfert des connaissances.

- Favoriser la recherche en proximité, pour établir des ponts solides et durables.

- Identifier avec les partenaires des modalités appropriées (c.-à-d. temps, ressources) afin de favoriser une meilleure expérience pour les recruteurs et leur permettre ainsi de rejoindre des préposés qui ne participent pas spontanément aux projets de recherche.

- Développer des outils de communication adéquats pour les préposés afin de les rejoindre plus efficacement dans le cadre des activités de recherche (c.-à-d. recrutement, activités de transfert de connaissances)

- Arrimer le développement d'interventions organisationnelles participatives avec le processus de transformations organisationnelles en cours afin d'optimiser son implantation, mais aussi sa portée. 


\section{Bibliographie}

Allaire É., É. Gagnon et Jauvin N. (2015). Symposium sur le métier des auxiliaires de santé et de services sociaux et des préposés aux bénéficiaires. Synthèse des présentations et des discussions, CSSS de la Vieille-Capitale, Québec.

Allaire É., N. Jauvin et Duchesne A. (2015). Démarche de prévention pour contrer les effets du travail émotionnellement exigeant chez les préposés auc bénéficiaires du CIUSSS de la Capitale-Nationale-Bilan des rencontres exploratoires, Québec: Centre intégré universitaire de santé et de services sociaux de la Capitale-Nationale.

Arborio A.-M. (1995). Quand le «sale boulot» fait le métier : les aides-soignantes dans le monde professionnalisé de l'hôpital. Sciences sociales et santé, 13 (3), 93-126.

Arborio A.-M. (1996). Savoir profane et expertise sociale. Les aides-soignantes dans l'institution hospitalière. Genèses, 22 (1), 87-106.

Arborio A.-M. (2012). Un personnel invisible. Les aides-soignantes à l'hôpital. $2^{e}$ édition Paris : Anthropos.

Aubry F. et Couturier Y. (2014a). Préposés aux bénéficiaires et aides-soignantes : entre domination et autonomie. Québec : Presse de l'Université du Québec.

Aubry F. et Couturier Y. (2014b). Regard sur une formation destinée aux préposés aux bénéficiaires au Québec. Gestion de contradictions organisationnelles et souffrance éthique. Travailler, 31 (1), 169-192.

Aubry F., Y. Couturier et Gilbert F. (2014). L'application de l'approche Milieu de vie en établissement d'hébergement de longue durée par les préposés aux bénéficiaires. Dans Préposés aux bénéficiaires et aides-soignantes : entre domination et autonomie (p.105-121), Presse de l'Université du Québec.

Bailey S., K. Scales, J. Lloyd, J. Schneider et Jones R. (2013). The emotional labour of health-care assistants in inpatient dementia care ». Ageing and Society, 35 (2), 246-269.

Bédard, S., et Bélanger., L. (2013). Analyse des données sur les accidents du travail en 2013. OP, 28, 14-15.

Bélanger, J. (1998). L'épuisement professionnel chez les préposés aux bénéficiaires oeuvrant en soins de longue durée (Mémoire, Université du Québec à Trois-Rivières, Trois-Rivières, Canada). Repéré à http://depot-e. uqtr.ca/4738/

Benelli N. et Modak M. (2010). Analyser un objet invisible : le travail de care. Revue française de sociologie, 51 (1), 39-60.

Bonnet M. (2006). Le métier de l'aide à domicile : travail invisible et professionnalisation, Nouvelle revue de psychosociologie, 1 (1), 73-85.

Bowers B.J., S. Esmond et Jacobson N. (2003). Turnover reinterpreted CNAs talk about why they leave, Journal og gerontological nursing, 29 (3), 36-44.

Côté, N. et Jauvin, N. (2014). RIPOST : une équipe de recherche en santé mentale au travail : Le partenaire, 22 (3), 2224.

Dubois F. (2014). L'aide-soignante en gériatrie ou l'organisation sociale des dégoûts, Dans Préposés aux bénéficiaires et aides-soignantes : entre domination et autonomie (p.159-173). 
Ministère du Travail, de l’Emploi et de la Solidarité sociale. (2017). Aides-infirmiers/aides infirmières, aides-soignants/aides-soignantes et préposés/préposées aux bénéficiaires (CNP 3413), Repéré à : http:// imt.emploiquebec.gouv.qc.ca/mtg/inter/noncache/contenu/asp/mtg122_descrprofession_01.asp?lang=FRAN\&pro=3413

Lhuilier D. (2005). Le «sale boulot». Travailler, 14 (2) : 73-98.

Marché Paillé A. (2011). Émotions et travail d'assistance aux soins personnels en gérontologie. Se garder du dégoût, mais pas trop (Thèse de doctorat, Université Laval, Québec, Canada).

Pfefferle S.G. et Weinberg D.B. (2008). Certified nurse assistants making meaning of direct care, Qualitative health research, 18 (7), 952-961.

Saillant F. et Gagnon É. (1999). Présentation. Vers une anthropologie des soins? Anthropologie et Sociétés, 23 (2), 5. 
TITRE: COMPRENDRE LA MARGINALITÉ OU RENFORCER LA MARGINALISATION?

RÉFLEXIONS ÉTHIQUES AUTOUR D'UNE RECHERCHE

Auteur(s): ÉRIC GAGNON

Publication: RECRUTEMENT ET CONSENTEMENT À LA RECHERCHE : RÉALITÉS ET DÉFIS ÉTHIQUES

PAgES: $98-105$

ISBN: 978-2-7622-0357-8

Directeurs: AnA MARIN, BÉATRICE EySERMANN ET MiChEL T. GIROUX

URI: HTTP://HDL.HANDLE.NET/11143/14114

DOI: HTTPS://DOI.ORG/10.17118/11143/14114 


\title{
Comprendre la marginalité ou renforcer la marginalisation? Réflexions éthiques autour d'une recherche
}

\author{
Éric Gagnon, chercheur, Centre de recherche sur les soins et les services de première ligne de l'Université Laval, Centre intégré uni-
} versitaire de santé et de services sociaux de la Capitale-Nationale

Résumé : Sur la base d'une expérience de recherche portant sur la marginalisation et l'exclusion de certaines catégories de personnes des espaces publics, l'auteur soulève un ensemble d'interrogations éthiques. La recherche participe des débats politiques et sociaux touchant la marginalité et les marginaux, leur réalité et les problèmes qu'ils occasionnent. Ce faisant, elle risque, bien malgré elle, de contribuer à créer et à renforcer la marginalisation, et de renforcer l'image négative que les personnes ont d'elles-mêmes. L'auteur avance quelques propositions pour prévenir la violence que la recherche pourrait ainsi produire ou contribuer à alimenter.

Mots clés : Marginalisation, identité, débats, violence, sujet. 
Il y a quelques années, dans le cadre d'une recherche sur le partage des espaces publics dans un quartier de la basse-ville de Québec, j'ai mené des entrevues avec des personnes dites marginales. Or, très souvent ces personnes me parlaient des marginaux à la troisième personne; elles ne s'identifiaient pas à la marginalité. D'ailleurs, les entretiens n'étaient parfois possibles que dans la mesure où elles n'étaient pas directement associées aux marginaux. Ces personnes avaient pourtant été recrutées par des intervenants de groupes communautaires œuvrant auprès des sans-abri, des prostituées, des pauvres et des toxicomanes. Elles devaient nous donner le point de vue des personnes marginalisées sur la manière dont elles cohabitent et interagissent avec les autorités et les autres groupes présents dans le quartier - marchands, résidents, policiers - de même que sur leur place dans les espaces publics, les parcs, le parvis de l'église, les devantures des commerces (Gagnon et coll, 2016; Parazelli et coll., 2013).

Pourquoi cet écart entre le statut de marginal qu'on leur attribuait et l'identité qu'elles se reconnaissaient? Sans doute ne voulaient-elles pas être associées à un groupe stigmatisé et à une condition dont elles cherchaient à échapper. Personne n'aime être identifié à des conduites mal perçues et des groupes ostracisés. Peut-être l'entretien leur permettait-il également de prendre une distance avec leur situation et de l'analyser : en parler à la troisième personne aurait été une manière de prendre du recul. Ce qui est certain, c'est que parler de la marginalité n'est jamais neutre. C'est jeter un regard sur une condition définie par la négative, l'isolement, le rejet, l'étrangeté, par des comportements condamnés pour leur caractère inconvenant ou menaçant. Si nous pouvons prendre une certaine distance avec le phénomène, nous ne pouvons jamais nous en détacher entièrement. La marginalité est en effet largement le produit du regard que l'on porte sur elle : le regard que les individus portent sur certaines conduites, modes de vie ou apparences; le regard que l'individu, ainsi regardé et jugé, pose sur lui-même. La marginalité est un jugement porté par un groupe sur un autre groupe, c'est l'image que des individus, par défi ou en réaction à ce jugement, renvoient aux autres. C'est être regardé ou être regardant, ou encore regardant-regardé. On ne peut en parler sans être pris dans ce jeu des regards, sans devoir se situer en dehors ou au-dedans de la marginalité, sans prendre position ni juger.

Pour la personne sollicitée par le chercheur, accepter de participer à une étude sur les marginaux signifie savoir qui sont les marginaux et ce qui fait leur marginalité, accepter d'en discuter, de clarifier ses idées sur la question, de porter un jugement, et donc de se compromettre. Pour certains participants c'est encore plus engageant, cela signifie accepter ou rejeter l'étiquette de marginal ou d'itinérant; il ne s'agit plus seulement de savoir ce qu'est la marginalité, mais de savoir qui l'on est ou qui l'on veut être, à ses propres yeux et aux yeux des autres. Les participants à l'étude sont tous personnellement concernés, mais de manières différentes; ils sont tous regardants et regardés, mais les conséquences sur leur vie ne sont pas les mêmes.

S'il est une question éthique importante dans les études sur la marginalité, c'est celle du respect de l'intégrité morale des personnes. La recherche ne doit pas leur renvoyer une image négative d'elles-mêmes, elle ne doit pas contribuer à leur exclusion, leur dévalorisation, leur sentiment d'étrangeté, leur expulsion des espaces publics ou leur silence. Si les principes d'autonomie et d'intégrité de la personne s'appliquent en ce domaine, ce n'est pas uniquement par l'obtention du consentement ou par la préservation de l'anonymat, mais dans le maintien chez ces personnes d'une capacité de prendre la parole, d'exprimer un point de vue et de s'adresser aux autres sans honte, ou sans provoquer chez les autres un sentiment de honte. Le chercheur ne peut pas ne pas s'interroger sur les répercussions de son étude sur les marginaux, sur la manière dont on les regarde et la manière dont ils se perçoivent. Il est pris dans le jeu des regards. 
La marginalité, comme tous les objets de la sociologie et des sciences sociales en général, fait débat. Avant que le sociologue ne s'en empare, elle fait l'objet de controverses. Elle est largement discutée et débattue dans les médias, les ministères, au Parlement; de nombreux groupes et individus se sont déjà prononcés sur sa nature, ses causes, ses conséquences et les mesures à prendre pour la contrer. C'est une réalité déjà largement interprétée (Gagnon, 2009). Le sociologue s'invite dans un débat qui a commencé avant lui et qui va se poursuivre après lui. Il ajoute son interprétation à celles qui circulent déjà, une interprétation qu'il veut plus large et plus complète, mieux fondée et argumentée ${ }^{1}$, et qui relance la discussion. Il se jette à son tour dans la mêlée.

L'objet «marginalité» ne va pas de soi. Sa définition, ses frontières et sa signification sont débattues, sa nature même est polémique. L'objet du sociologue n'est d'ailleurs pas tant la marginalité en soi que le débat sur la marginalité. L'étude à laquelle j'ai fait allusion plus haut avait précisément pour objet un débat : le partage des espaces publics entre les personnes marginalisées et les autres populations fréquentant ou habitant le même quartier. Nous devions apporter un éclairage à une controverse qui existait déjà : les commerçants se plaignaient de la présence de certaines personnes dites «marginales» à proximité de leur commerce, des organismes communautaires prenaient la défense de ces personnes, les autorités municipales tentaient d'arbitrer le différend entre les commerçants et les groupes, et la police menait diverses interventions, tantôt répressives, tantôt apaisantes. Tout un chacun avait son explication quant à la nature et la source du problème, et le gouvernement provincial, qui avait financé notre étude, était à la recherche de solutions. Étudier la marginalité, ici comme ailleurs, c'est d'abord rendre compte d'un débat, c'est faire entendre des voix, rendre visibles des conduites, faire apparaître des jugements; en un mot : confronter les divers regards et poser à son tour un regard.

Dans une étude sur la marginalité, les questions épistémologiques, éthiques et politiques sont ainsi inextricablement liées. En définissant la marginalité, en cherchant à circonscrire le phénomène et à le caractériser, le chercheur et ses interlocuteurs (ou informateurs) sont conduits simultanément à se prononcer:

1. sur la perspective dans laquelle la marginalité doit être comprise, sa nature, ses causes, ses conséquences (questions épistémologiques);

2. sur l'identité du marginal, ce à quoi on le reconnaît, et donc sur le regard que l'on pose sur lui - son image, son égale dignité, le respect qu'on lui doit, l'importance accordée à son point de vue (questions éthiques);

3. sur les politiques à adopter - répressives ou pas -, sur les règles à faire respecter et la place accordée aux conduites marginales dans la société (questions politiques).

1. C'est ce qui différencie une interprétation sociologique d'une idéologie : comme tout discours scientifique, l'interprétation du sociologue se veut argumentée et son auteur accepte de la soumettre à la critique en exposant ses arguments et en se soumettant à l'évaluation par les pairs (colloques et publications). Elle prétend également rendre compte d'un plus grand nombre de dimensions du phénomène étudié que les autres discours. 
Les questions épistémologiques, éthiques et politiques ne se séparent pas; elles sont plutôt les trois faces d'une même préoccupation ou interrogation : Quels sont les rapports que les divers groupes sociaux entretiennent avec la marginalité et le marginal, quels sont les rapports qu'ils devraient entretenir avec eux?

La question du respect de l'intégrité morale des participants à la recherche s'inscrit dans une question éthique aux plus larges dimensions touchant la participation du chercheur et des participants à un débat moral et politique. La question n'est pas simplement déontologique (préserver l'anonymat des personnes dans le recueil des données et l'exposé des résultats), elle consiste à entrer de plain-pied dans un débat public, y faire entrer d'autres personnes - les marginaux, les commerçants, les résidents - en faisant entendre leur voix, en suscitant leur réflexion et en les obligeant à se regarder, à mettre en lumière les différentes dimensions du problème. La question éthique pour le chercheur est de décider à qui il va donner la parole, comment il va la donner et ce qu'il va faire de ce qu'il a entendu.

\section{III}

Cette question, aux dimensions tant épistémologiques, éthiques que politiques, se pose au chercheur à toutes les étapes de son étude.

1. Elle se pose au tout début de son enquête au moment de formuler sa question de recherche. Déjà, il lui faut retenir certains critères par lesquels il reconnaît la marginalité afin de pouvoir identifier des conduites sur lesquelles il interrogera diverses personnes. Même s'il se garde alors de porter explicitement un jugement sur ces individus ou ces conduites, il les désigne comme faisant problème pour d'autres. Il sélectionne parmi différents problèmes débattus dans la société ceux qui doivent retenir l'attention, et cela pourra inclure autant les conduites marginales que les conduites de réprobation ou d'exclusion à l'endroit des marginaux. La manière dont le problème est formulé et la perspective retenue (et conséquemment d'autres perspectives rejetées) reposent sur des choix théoriques, mais aussi éthiques et politiques sur ce qui mérite attention, et qui ne seront pas sans conséquence sur le regard que les participants seront amenés à poser sur les autres et sur eux-mêmes.

2. Cette question éthique demeure présente au moment d'approcher et de recruter des personnes pour un entretien ou répondre à un questionnaire. Comment faut-il les aborder? À quel titre le chercheur va-t-il leur demander de participer à l'étude? En tant que personne marginalisée, ou à titre d'usager d'un centre d'aide pour toxicomanes, sans préjuger de leur marginalité? En tant que résident ou commerçant du quartier ou comme personne demandant l'intervention de la police ou l'adoption de nouvelles réglementations municipales visant à réduire la présence des marginaux? À quel titre, en vertu de quelles connaissances ou de quelle compétence, au nom de quels intérêts ou de quelle autorité vont-elles parler? Quelle position dans le débat leur demandera-t-il d'occuper? Cette position ne sera pas sans conséquence sur la légitimité de leurs discours et de leur participation au débat.

3. Dans la manière dont les entretiens seront conduits, la question va continuer de se poser. Les thèmes retenus, la formulation des questions, jusque dans l'attitude et les réactions de l'intervieweur face aux propos tenus par la personne interrogée, auront une incidence - minime parfois, forte à d'autres moments - sur la manière dont cette personne va se percevoir et se sentir jugée, sur sa compréhension du problème, sur la légitimité de certaines conduites, la sienne, celle des autres (autant la conduite marginale que la conduite répressive ou d'exclusion). Comprenons bien ici que le fait qu'un individu soit amené à réfléchir sur sa conduite, à la voir autrement, à se remettre en question n'est pas en soi un problème. Nous avons affaire à des adultes qui ont librement consenti à discuter d'un problème et à répondre à des questions. C'est souvent même l'un 
des objectifs de l'étude que de susciter pareille remise en question, tant chez les marginaux que chez les commerçants ou les policiers. Après tout, il s'agit de faire avancer le débat. Le chercheur doit cependant savoir que ce qu'il fait n'est pas neutre ni sans effet, et que, si ces effets ne sont pas entièrement contrôlables, il lui faut minimiser les plus indésirables (honte, gêne, mutisme).

4. Enfin, cette question se pose au moment de communiquer les résultats de la recherche lorsqu'il s'agit de choisir ses mots pour une conférence ou un article. Comment décrire la situation, rendre compte du débat, présenter les positions et caractériser les comportements de chacun, sachant qu'il n'y a pas de manière neutre de le faire? Quelle image le chercheur laisse-t-il des individus? Comment fera-t-il droit à leur parole et à leur point de vue (sans nécessairement les endosser)? Il faut de toute évidence peser ses mots. Là encore, ça ne veut pas dire éviter toute réaction ou tout débat, au contraire, mais éviter de blesser ou de vexer inutilement. Il faut aussi être prêt à défendre sa lecture des évènements et des problèmes, montrer ce qu'elle ajoute, ce qu'elle permet de faire entendre ou de faire voir, sans pour autant faire taire d'autres voix ou masquer d'autres réalités. Elle doit élargir la compréhension du débat, accroître la vision, la portée des regards.

Le choix des mots, l'image ou l'interprétation produite nous ramènent au recrutement des sujets de recherche. Lorsqu'une personne marginalisée a accepté de répondre à nos questions, elle nous a fait confiance, elle a pensé que cela ne pouvait pas lui nuire, et peut-être que cela pourrait même améliorer sa situation. II en est de même pour le commerçant ou le résident qui se plaignent de la présence des marginaux. En acceptant de répondre à nos questions, il nous a fait confiance lui aussi : son point de vue et ses arguments allaient être entendus, pris au sérieux, sérieusement discutés, et non simplement écartés ou condamnés. Le chercheur s'est engagé à ne pas traiter ce dernier simplement comme une personne intolérante et répressive, aux comportements moralement condamnables. En le sollicitant, le chercheur a pris un engagement - explicite ou tacite - à respecter qui il est, ce qu'il pense et ce qu'il fait. Le chercheur ne peut l'oublier.

\section{IV}

Je terminerai par trois remarques. La première est que la recherche en sciences sociales peut être violente. Par les représentations des individus et des situations qu'elle produit ou reproduit, elle peut contribuer à entretenir un sentiment d'infériorisation, d'insignifiance ou d'impuissance, raviver aussi des blessures. Par les interprétations qu'elle avance, elle peut contribuer à réduire ou à durcir les différences, à renforcer ou à atténuer l'exclusion sociale, à donner la parole ou à imposer le silence. Comme tout regard, elle peut renvoyer aux autres une image négative d'eux-mêmes, leur enlever toute volonté ou toute capacité de prendre la parole, de se raconter ou d'agir, et ainsi réduire leur autonomie². Les savoirs peuvent être la source d'une réelle violence. Sans doute faut-il en prendre le risque. Se taire, ce serait faire perdurer une situation ou des préjugés. L'éthique ne consiste pas ici à tout faire pour éviter d'entrer dans le débat et de remuer les esprits, mais à y entrer prudemment.

La seconde remarque porte sur la perspective d'analyse. Plutôt que de se centrer sur les individus, il faut se centrer sur les rapports que ces individus entretiennent. Il faut étudier les processus de marginalisation plutôt que les marginaux; des processus qui se traduisent dans des jugements, des conduites d'évitement ou d'expulsion, de défi ou de mise à distance, mais aussi des représentations, des règlements et des aménagements urbains. Ce changement de perspective permet d'abord de ne pas fixer l'attention sur les individus, leur mode de vie ou leur conduite, et de prévenir les jugements trop rapides à leur endroit. II permet ensuite de faire avancer la réflexion, d’élargir la vision en se plaçant au niveau des rapports entre les individus plutôt que les individus

2. Je reprends la définition de l'autonomie proposée par Ricoeur (2001) qui se caractérise par la triple capacité de dire, de faire et de se raconter. 
considérés séparément. Ce déplacement du regard permet de penser l'altérité, d'interroger les différences et les distances que les différents groupes établissent entre eux, de questionner les écarts et les antagonismes que produisent ces différences, de comprendre l'inquiétude, les peurs et la méfiance que ces distances entretiennent. Ce déplacement permet également de prendre une distance avec nos manières de voir et de penser, d'avoir accès à d'autres points de vue, non pour les adopter et s'y installer, mais pour en saisir le sens et les raisons. Il permet de réduire l'altérité sans l'abolir, de réduire les différences en les rendant compréhensibles, sans pour autant les effacer.

La troisième remarque touche au débat social. En changeant la perspective, la sociologie contribue non seulement à élargir la compréhension du phénomène, mais également à élargir la participation au débat. Elle permet de donner la parole et d'écouter ce qui se dit, de faire entendre une pluralité de voix, au premier chef celles qu'on entend peu. Elle permet à une diversité de personnes et de groupes de participer à l'interprétation du problème, à sa compréhension et à sa transformation. Sans faire disparaître les divisions et les conflits, elle donne la possibilité à tous de faire entendre ses raisons, mais aussi d'opérer un déplacement, de modifier le regard qu'ils portent sur les autres. Le terme sujets pour désigner les participants à une étude prend alors tout son sens. Son emploi est rarement aussi significatif et conséquent que dans la recherche sur la marginalité et les marginaux. 


\section{Bibliographie}

Gagnon, É. (2009). Politique du cercle. Interprétation, éthique et sociologie, Cahiers de recherche sociologique, 48, 15-26.

Gagnon, É, M. Parazelli et Hardy, M.-H. (2016). Visibles et invisibles. Marginalités et partage des espaces publics à Québec. Dans D. Lamoureux et F. Dupuis-Déri (dir.) Au nom de la sécurité ! Criminalisation de la contestation et pathologisation des marges (p. 113-129), Montréal : M éditeur.

Parazelli M. et coll. (2013). Les enjeux du partage de l'espace public avec les personnes itinérantes et sa gestion à Montréal et à Québec. Perspectives comparatives et pistes d'actions. Fonds de recherche du Québec -Société et culture, Rapport de recherche Programme actions concertées, 19 août 2016, [version électronique]. http://www.frqsc.gouv.qc.ca/documents/11326/448958/PC_PazarelliM_rapport+2013_espaces+publics+vs+itinérance/

Ricœur, P. (2001). Le juste 2, Paris : Éditions Esprit. 
TITRE: RECHERCHE SUR LA SPIRITUALITÉ : LES DÉFIS DU RECRUTEMENT

Auteur(s): Bruno BÉLANGER ET Line BEAUREgARD

Publication: ReCRUtemEnT ET CONSENTEMENT À LA RECHERCHE : RÉALITÉS ET DÉFIS ÉTHIQUES

PAgES: $106-114$

ISBN: 978-2-7622-0357-8

Directeurs: Ana Marin, BéATrice Eysermann et Michel T. GIROUX

URI: HTTP://HDL.HANDLE.NET/11143/14115

DOI: HTTPS://DOI.ORG/10.17118/11143/14115 


\section{Recherche sur la spiritualité : les défis du recrutement}

Bruno Bélanger, agent de planification, de programmation et de recherche au Centre Spiritualitésanté de la Capitale-Nationale (CSsanté) du CHU de Québec - Université Laval.

Line Beauregard, agente de planification, de programmation et de recherche au Centre Spiritualitésanté de la Capitale-Nationale (CSsanté) du CHU de Québec - Université Laval. Membre de l’Ordre des psychologues du Québec.

Résumé : Au cours des dernières années, le CSsanté a réalisé des projets de recherche afin de mieux comprendre comment, dans l'environnement social pluraliste et laïque du Québec contemporain, se manifestait l'expression spirituelle de personnes en contexte de maladie. À partir de deux recherches réalisées auprès d'usagers en soins palliatifs, les auteurs font ressortir les défis rencontrés lors du recrutement des participants. Plusieurs de ces défis sont reliés au thème des recherches qui portaient sur les besoins spirituels et religieux des patients en fin de vie. II appert que l'utilisation des mots «spirituels» et «religieux» pouvait évoquer, tant pour les patients que pour les soignants, une recherche sur la foi telle que comprise dans la tradition judéo-chrétienne, ce qui a pu limiter la participation de personnes n'adhérant pas à ce courant. Afin d'obtenir des résultats plus représentatifs de la diversité des expériences spirituelles, il aurait été plus adéquat de présenter le projet comme une recherche portant sur le vécu intérieur en contexte de maladie.

Mots clés : spiritualité, soins palliatifs, recrutement, recherche, besoins spirituels

Summary: In the course of the last several years, the CSsanté produced several research projects so as to better understand how, within the pluralist and secular social environment of contemporary Québec, does spirituality manifest itself for persons within a context of illness. On the basis of two research studies conducted with clients in palliative care, the authors bring to the fore the various challenges which they encountered in their attempts to recruit participants. Several of these challenges were related to the research topics in question which dealt with the spiritual and religious needs of patients who were at the "end of life" phase of their lives. It would appear that the use of such words as "spiritual and religious" often evoked, for the patients as well as the caregivers, an inquiry into matters of faith such as is understood within a Judaeo-Christian tradition, which, it was found, often limited the participation of persons who did not adhere to this stream of consciousness. In an effort to obtain results which better represent the diversity of spiritual experiences in contemporary society, it would have been more appropriate, to present this project as a vehicle for research dealing with the inner lived experience of persons struggling with illness.

Keywords: spirituality, palliative care, recruitment, research, spiritual needs. 


\section{Pourquoi une recherche sur la spiritualité?}

Le Centre Spiritualitésanté de la Capitale-Nationale (CSsanté) s'investit depuis une douzaine d'années dans la recherche. La nécessité d'améliorer nos connaissances en nous appuyant notamment sur des recherches s'est accrue à mesure que se faisaient sentir, au sein du réseau de la santé, les conséquences des changements socioreligieux véhiculés par la Révolution tranquille. En effet, les professionnels impliqués à l'époque comme animateurs de pastorale ${ }^{2}$ prenaient progressivement conscience de transformations dans l'expression des besoins spirituels et religieux des patients et, par conséquent, de l'importance d'ajuster les modes d'intervention préconisés jusqu'à ce jour. Enfin, à l'époque des publications de plus en plus nombreuses, issues tout particulièrement de milieux anglophones, apparaissaient dans les champs situés à l'intersection de la spiritualité et de la santé.

C'est dans ce contexte que le CSsanté a souhaité mieux cerner comment, dans un environnement pluraliste et laïque, se manifestaient les grandes questions ou expressions de l'expérience spirituelle des patients. En d'autres mots, même si extérieurement de plus en plus de personnes n'adhéraient plus aux croyances véhiculées par une tradition spirituelle et une Église, elles continuaient de porter un ensemble de questions, de craintes, de sentiments reliés à leur vie intérieure. À travers ces recherches, nous espérions une meilleure compréhension de ce que vivent les patients en regard de leur vie spirituelle afin d'améliorer nos processus d’accompagnement dans la dernière étape de leur vie.

L'article qui suit mettra en lumière les principaux défis rencontrés lors du recrutement de patients dans le cadre de deux recherches qualitatives mises sur pied par le CSsanté entre 2007 et 2014 : une première étude concernant les patients suivis en soins palliatifs à domicile et une deuxième en soins palliatifs en milieu hospitalier. Ces recherches avaient deux objectifs spécifiques: 1) explorer et identifier les besoins spirituels et religieux des personnes en soins palliatifs; 2) élaborer une grille d'évaluation des besoins spirituels et religieux.

\section{Description du recrutement}

Concernant l'Étude portant sur les patients suivis à domicile, la collecte des données s'est effectuée sur le territoire du Centre de santé et de services sociaux (CSSS) de la Vieille-Capitale ${ }^{3}$. Trois équipes d'intervenants secteur Basse-Ville-Limoilou-Vanier, secteur Haute-Ville-des-Rivières, secteur Sainte-Foy-Sillery-Laurentien - ont été sollicitées pour proposer aux usagers de participer au projet de recherche.

Comme condition préalable à leur participation à l'étude, les personnes devaient être inscrites à un programme de soins palliatifs à domicile dispensé par un Centre local de services communautaires. Vingt-quatre entrevues ont ainsi été réalisées par deux interviewers sur une période de quatorze mois, soit de mai 2007 à juillet 2008.

1. Le Centre Spiritualitésanté de la Capitale-Nationale (CSsanté) a pour mission d'assurer la planification, la coordination, la prestation et l'évaluation d'activités cliniques de qualité afin de répondre aux besoins spirituels et religieux des personnes hospitalisées, hébergées ou suivies à domicile. Il regroupe tous les services de soins spirituels des établissements de santé et de services sociaux de la ville de Québec et de ses environs situés sur le territoire de la Capitale-Nationale. Composée de plus de 40 intervenants en soins spirituels, notre équipe de professionnels se déploie sur 35 sites répartis dans trois établissements du réseau de la santé et des services sociaux.

2. Au moment où nous avons entamé les recherches, le titre d'emploi des intervenants en soins spirituels était « animateur de pastorale».

3. Depuis l'entrée en vigueur de la projet de loi 10, le CSSS de la Vieille-Capitale est intégré au CIUSSS de la Capitale-Nationale. 
En tant que patients intégrés dans un programme de soins palliatifs, tous les participants avaient été informés par leur médecin, quelque temps avant leur participation à la recherche, qu'ils avaient une espérance de vie de moins de six mois, bien que certains aient pu ne pas avoir encore complètement assimilé cette information.

Pour la recherche auprès de patients en milieu hospitalier, les données ont été recueillies auprès de la clientèle en soins palliatifs uniquement à L'Hôtel-Dieu de Québec. Comme condition préalable à leur participation à l'étude, les personnes devaient être hospitalisées en soins palliatifs à L'Hôtel-Dieu de Québec. Entre juillet 2011 et avril 2013 (21 mois), 15 entrevues ont été réalisées au chevet des patients.

Pour les deux recherches, les intervenants ont présenté aux personnes sélectionnées un dépliant décrivant les objectifs et les modalités de leur participation au projet. Lorsqu'une personne acceptait de participer, l'intervenant transmettait son nom à un chercheur qui entrait rapidement en contact avec elle pour discuter plus en détail du projet et de ses implications et une rencontre était alors planifiée.

\section{Les participants}

Le tableau 1 présente la répartition des participants rencontrés à domicile selon leur groupe d'âge et selon leur sexe : seize femmes et huit hommes dont l'âge varie de 52 à 88 ans. Deux fois plus de femmes que d'hommes ont participé à la recherche et le groupe d'âge où l'on retrouve davantage de participants est celui des 69 ans et moins (Charboneau et Bélanger, 2013).

Tableau 1. Répartition des participants à domicile selon le genre et l'âge

\begin{tabular}{|l|l|l|l|}
\hline & Hommes & Femmes & Total \\
\hline 80 ans et plus & 2 & 4 & 6 \\
\hline 70 à 79 ans & 4 & 4 & 8 \\
\hline 69 ans et moins & 2 & 8 & 10 \\
\hline Total & 8 & 16 & 24 \\
\hline
\end{tabular}

Le tableau 2 présente la description des participants rencontrés en milieu hospitalier selon les mêmes critères que le tableau précédent. On remarque que le groupe d'âge où l'on retrouve davantage de participants est, comme à domicile, celui des 69 ans et moins; la répartition entre les hommes et les femmes est presque égale (Bélanger et Charbonneau, 2014).

Tableau 2. Répartition des participants en milieu hospitalier selon le genre et l'âge

\begin{tabular}{|l|l|l|l|}
\hline & Hommes & Femmes & Total \\
\hline 80 ans et plus & 1 & 1 & 2 \\
\hline 70 à 79 ans & 2 & 1 & 3 \\
\hline 69 ans et moins & 5 & 4 & 6 \\
\hline Total & 8 & 6 & 9 \\
\hline
\end{tabular}




\section{Les défis du recrutement}

Ces deux recherches ont posé des défis particuliers reliés au recrutement des participants. Nous les avons regroupés en six points : 1) élaborer le dépliant; 2) évoquer le mot «spiritualité»; 3) joindre les participants potentiels; 4) prendre rendez-vous; 5) amorcer l'entrevue; 6) distinguer les rôles de chercheur et d'intervenant.

\section{Défi un : Élaborer le dépliant}

Selon nous, la spiritualité ne réfère pas à une «chose» qu'on pourrait mettre à distance, mais avant tout à une posture d'écoute 4 . Selon cette manière d'envisager la spiritualité, nous voulions que les patients racontent, le plus librement possible, ce que l'expérience de la maladie et l'approche de la mort remuaient en eux. Ensuite, à partir de leur discours, nous souhaitions discerner leurs besoins spirituels et religieux. Nous avions opté pour un dépliant qui exprimait en quelques mots assez larges ce que nous cherchions à entendre : «Les questions qui vous préoccupent, vos ressources intérieures, l'importance de vos relations, vos craintes et vos joies.» Par ailleurs, et ce fut probablement une erreur, le dépliant indiquait aussi que la recherche était menée par notre service - à l'époque le Centre de pastorale de la santé et des services sociaux (CPSSS) $)^{5}$ et que l'objectif était de cerner les besoins spirituels et religieux des patients. Sans que nous le voulions, le document pouvait évoquer une démarche s'adressant particulièrement aux personnes ayant la foi ou adhérant ouvertement à une religion. Ainsi, malgré une description limpide de ce que nous attendions des patients, le choix d'inscrire le nom de l'organisation responsable et l'objectif final de la recherche a pu avoir comme conséquence de biaiser le recrutement.

\section{Défi deux : Évoquer le mot «spiritualité»}

Même si effectivement il était clair que ce que nous cherchions à entendre était de l'ordre de leur vie intérieure, de ce qu'ils vivaient, dès lors que les mots «spirituels» et «religieux» étaient imprimés sur notre dépliant, cette recherche prenait, dans la tête de plusieurs, une orientation d'abord religieuse. Également, même si l'accent était globalement placé sur la dimension spirituelle, il n’en demeure pas moins que des préjugés tenaces, au sein de la population en général, identifient encore étroitement spiritualité et religion. Notre recherche n'a pas échappé à cela! Plusieurs patients s'attendaient pratiquement à une rencontre avec un représentant d'une religion, et il n'était pas évident pour l'intervenant qui présentait le projet (prochain défi) de parler de la spiritualité sans référer à des concepts religieux. Conséquemment, il a fallu souvent repositionner, dès le début des entretiens, dans un langage très accessible, ce que nous entendions par «spiritualité» dans le cadre de cette recherche et ce que nous cherchions vraiment à saisir dans leur parcours de la maladie : non pas leur positionnement en regard d'un dieu ou d'une église, mais leur vie intérieure (ce qui pouvait bien sûr les amener à parler de Dieu ou de leur appartenance à une tradition religieuse, mais dans un second temps seulement).

\footnotetext{
4. Cette réflexion de Rosselet (2015) rend bien compte de cette réalité : « C'est d'ailleurs, selon mon expérience, l'une des caractéristiques de la spiritualité que de se vivre et se dire dans des aspects très concrets et simples de l'existence, mais qui ouvrent sur des hauteurs vertigineuses et des profondeurs abyssales! L'intervenant en soins spirituels, comme tout soignant d'ailleurs, sera attentif à écouter à l'arrière-fond des mots les plus simples les harmoniques spirituelles fondamentales qui les sous-tendent ; il entendra derrière les mots du quotidien le trouble et la quête spirituels qui s'y révèlent. » (p. 19).
}

5. Notre organisation a, suite à un nouveau cadre ministériel régissant la profession, changé de nom en 2011 pour devenir le Centre Spiritualitésanté de la Capitale-Nationale (CSsanté). Au moment où le dépliant a été élaboré, nous portions le nom de Centre de pastorale de la santé et des services sociaux (CPSSS). 


\section{Défi trois : Joindre les participants potentiels}

Puisque nous n'étions pas autorisés par notre CER à communiquer directement avec les participants potentiels pour les solliciter à participer au projet de recherche, nous avons dû passer par un intermédiaire. Le rôle de l'intermédiaire consistait à demander aux participants potentiels s'ils acceptaient qu'un membre de l'équipe de recherche entre en communication avec eux. Dans le cas des deux projets de recherche en question ici, ce sont des intervenants liés à l'équipe de soins qui ont fait ce premier contact. Il est devenu manifeste, après quelques entrevues, que les personnes qui avaient accepté de participer étaient presque toutes croyantes et pratiquantes, ce qui n'était pas représentatif de la diversité habituelle des patients rencontrés par les intervenants en soins spirituels (ISS). Était-ce le dépliant informatif qui biaisait le recrutement (comme nous l'avons évoqué plus haut)? Ou bien la manière dont il était présenté? Nous reconnaissons que le dépliant aurait dû être différent, mais émettons aussi l'hypothèse que la vision de l'intervenant qui réalisait ce premier contact a également joué un rôle de première importance. Si sa compréhension du thème de l'étude se résumait à une recherche sur la foi telle que comprise dans la tradition judéo-chrétienne, il est fort probable qu'il ait présenté cette recherche à des personnes qu'il savait ou pressentait être croyantes. Un intervenant avait d'ailleurs dit : «On pourrait demander à ce patient, je sais qu'il est croyant.» Il a donc fallu réexpliquer à quelques reprises aux intervenants le sens de notre recherche.

\section{Défi quatre : Prendre rendez-vous}

Après avoir obtenu l'accord d'un patient, l'intervenant transmettait au chercheur le nom, le numéro de téléphone et les autres informations de base (âge et diagnostic) le concernant. Le patient était joint par téléphone et nous fixions un moment pour la réalisation de l'entrevue. Cette étape a souvent constitué pour nous un autre défi. Malgré le fait que le patient ait donné son accord à l'intervenant, bien des événements pouvaient survenir entre les heures (ou les jours) qui séparent l'acquiescement verbal du patient et le téléphone du chercheur : maladie soudainement aggravée, humeur différente, visite imprévue, etc. Avec certains patients, le chercheur devait trouver le juste équilibre entre dénouer les craintes ou les appréhensions bien normales du patient d'une part, et, d'autre part, ne pas trop insister si le patient ne sou haitait plus se prêter à la recherche. Comme il est souvent difficile de recruter, le chercheur se devait d'être vraiment attentif à cette réalité afin de ne pas s'imposer. Il s'agissait ensuite de trouver le moment opportun pour la rencontre. En milieu hospitalier notamment, la dernière étape de vie se compte en nombre de jours. Par conséquent, la rencontre doit être très rapprochée, et il est essentiel de trouver le moment de la journée - ce dernier point est vite apparu fort important, car les temps libres peuvent être rares. En effet, ceci est déterminant puisque le chercheur doit s'assurer que le climat dans lequel l'entrevue se déroule est optimal, favorisant le partage de propos intimes et parfois très profonds.

\section{Défi cinq : Amorcer l'entrevue}

Nous avons constaté que l'étape du recrutement ne se termine pas forcément à la prise du rendez-vous, les débuts de l'entrevue font aussi partie du processus. En effet, les premières minutes de la rencontre, consacrées à la vérification de la bonne compréhension du patient en regard de sa participation et de la signature du feuillet de consentement, sont cruciales pour tout projet de recherche. Le nôtre n'a pas échappé à cette réalité. 
Voici un exemple significatif à cet égard. L'interviewer est entré chez une dame d'environ 70 ans manifestement communicative et qui, dès son arrivée, commence à partager un segment fort difficile de sa jeunesse. L'interviewer était déchiré entre le respect qu'il portait à ce qu'elle racontait avec beaucoup d'émotions (quelques larmes glissaient régulièrement sur ses joues) et son obligation de lui dire : «Je suis contraint, madame, de vous faire signer les formulaires de consentement et de vous informer à nouveau que je dois procéder à l'enregistrement de notre entretien». Dans ce cas, la signature a finalement pu être réalisée, mais il a été difficile de retrouver le rythme et la profondeur de la discussion de départ. Dans les premiers moments de la rencontre, l'interviewer avait même envisagé, pendant quelques minutes, de ne pas réaliser cette entrevue de recherche selon les critères éthiques convenus. Dans ce contexte aurait-il été éthiquement acceptable de laisser parler la personne, de l'enregistrer et, à la fin, de lui présenter le formulaire d'information et de consentement (FIC)? Cet exemple à notre sens illustre les défis qui peuvent toujours se présenter, même quand les principaux obstacles du recrutement semblent surmontés.

Ensuite, même après l'étape de la signature du FIC dûment terminée, il peut encore survenir une remise en question de la poursuite de l'entrevue. Par exemple, lors des premières questions, un autre participant avait évoqué discrètement une journée plus difficile et un manque d'énergie, sous-entendant qu'il n'était peut-être plus convenable de continuer l'entrevue. Il est vite apparu essentiel de porter attention à ces quelques mots indiquant un malaise et de signaler au patient, à nouveau, que nous n'attendions rien d'autre que sa parole à lui, qu'il n'y avait pas de bonnes ou de mauvaises réponses et qu'à tout moment il pouvait mettre fin à l'entretien. Il faut donc savoir entendre ce que le patient dit, même si c'est dit, quelquefois, indirectement. Parfois, simplement accepter de prendre le temps de bien écouter dénoue les craintes en regard de l'entrevue.

\section{Défi six : Distinguer les rôles de chercheur et d'intervenant}

Un dernier défi rencontré a souvent consisté à discerner si le patient avait bien saisi la différence entre le rôle de chercheur et celui d'intervenant en soins spirituels. Le simple mot «spiritualité», lors de la présentation du projet de recherche, suffisait, pour un certain nombre de patients, à dissocier notre approche d'une recherche ayant un caractère objectif et neutre. Bon nombre nous recevait comme si nous étions des ISS. Nous avons rapidement saisi qu'il était essentiel de présenter dans des mots simples et concrets ce qu'est un chercheur, ses objectifs, ses limites et ce que pourrait vivre avec eux un intervenant en soins spirituels dans une rencontre subséquente. Faire cette distinction est fondamental, puisque le contenu de l'entrevue peut s'avérer assez différent selon que le patient pense s'adresser à un chercheur ou à un ISS. De plus, ses attentes peuvent ne pas être satisfaites.

\section{Conclusion}

Enfin, un dernier aspect mérite d'être mentionné. L'entrevue, compte tenu du type de questions évoquées, pouvait mettre en lumière certaines souffrances chez la personne en regard de cette ultime étape de sa vie. Le chercheur pouvait constater, dans certains cas, qu'un patient gagnerait à être correctement accompagné par un professionnel des soins spirituels. Mais, pour différentes raisons (probablement des préjugés tenaces en regard de la religion que nous avons évoqués plus haut), certains participants ont manifesté clairement ne pas vouloir s'inscrire dans un tel processus. Bien que les participants aient exercé leur droit de refus, les laisser dans cet état de souffrance, suite aux questions d'entrevue, pouvait soulever pour le chercheur un certain malaise. 
Avec le recul, il est manifeste pour nous que, si nous avions à reprendre cette recherche, nous procéderions différemment en ce qui a trait au recrutement. Les termes «spiritualité» et «religion» ne seraient pas mentionnés dans notre dépliant, nos documents et nos présentations aux intervenants chargés du recrutement afin d'éviter les préconceptions liées à ces termes. II serait préférable de parler d'une recherche qui porte sur l'expérience intérieure dans le contexte de la maladie afin de rejoindre tous les patients, peu importent leurs croyances et leurs a priori face à la religion et la spiritualité. Nous serions ainsi en accord avec notre vision de la spiritualité et avec ce que nous voulons vraiment repérer : la prise de parole du patient sur ce qui se joue en lui, c'est-à-dire ses préoccupations, ses craintes, ses moments de bonheur, ses questions, etc. En fin de compte, les résultats de l'analyse du contenu des entrevues nous permettraient quand même de cerner leur expérience spirituelle. De plus, nos résultats seraient fort probablement plus représentatifs de la diversité des patients rencontrés dans le réseau de la santé et des services sociaux. 


\section{Bibliographie}

Bélanger, B. et Charbonneau, C. (2014). Reconnaître et accompagner l'expérience spirituelle en fin de vie. Les Cahiers francophones de soins palliatifs, 14 (1), 47-56.

Charbonneau, C. et Bélanger, B. (2013). Identification of spiritual and religious needs of terminally ill patients receiving palliative home care. Journal for the Study of Spirituality, 3 (1), 33-45.

Rosselet, F. (2015). La souffrance spirituelle | De l'enfermement à l'ouverture d'un espace. Spiritualitésanté, 8 (3), 18-21. 
TITRE: LES PROBLÉMATIQUES DU RECRUTEMENT AUPRÈS DES PATIENTS ATTEINTS DE TRAUMATISMES CRANIOCÉRÉBRAUX (TCC) ET D’ALZHEIMER

Auteur(s): Kim Charest et Julie Bouchard

PublicATION: RECRUTEMENT ET CONSENTEMENT À LA RECHERCHE : RÉALITÉS ET DÉFIS ÉTHIQUES

PAGES: $115-126$

ISBN: 978-2-7622-0357-8

Directeurs: Ana Marin, BÉatrice Eysermann et Michel T. Giroux

URI: HTTP://HDL.HANDLE.NET/11143/14182

DOI: HTTPS://DOI.ORG/10.17118/11143/14182 


\section{Les problématiques du recrutement auprès des patients atteints de traumatismes craniocérébraux (TCC) et d'Alzheimer}

Kim Charest, étudiante au doctorat en neuropsychologie, Université du Québec à Montréal Julie Bouchard, Ph. D., directrice de doctorat en psychologie, neuropsychologue et professeure, Université du Québec à Chicoutimi

Résumé : L'expérimentation de nouvelles technologies d'assistance et d'intelligence artificielle s'avère nécessaire à l'augmentation de l'autonomie et de l'indépendance des populations vulnérables. Notamment, les patients ayant subi un traumatisme craniocérébral (TCC) et ceux atteints de la maladie d'Alzheimer sont susceptibles de pouvoir bénéficier de tels outils technologiques au quotidien. En raison des difficultés motrices, des problèmes cognitifs et des troubles de mémoire présents dans ces populations, les différentes étapes des recherches en sont complexifiées, particulièrement en matière de recrutement. Les expérimentations menées au Laboratoire d'intelligence ambiante pour la reconnaissance d'activités (LIARA) ont permis de souligner plusieurs problématiques éthiques liées à la recherche auprès de ces populations. D’autre part, ces études ont également mené à une panoplie de solutions simples et efficaces afin de travailler avec les proches, d'obtenir un consentement valable et d'éviter les enjeux liés aux problèmes cognitifs.

Mots clés : recrutement de participants, technologies d'assistance cognitive, consentement éclairé, troubles cognitifs. 


\section{Introduction}

Le recrutement de certaines clientèles pour la participation à un projet de recherche est en soi une expérience. Lorsque les participants visés par un projet ont des atteintes cognitives et/ou sont déclarés inaptes, faire preuve de créativité, autant pour le recrutement que pour la participation concrète, est essentiel. Les recherches qui sont effectuées au Laboratoire d'intelligence ambiante pour la reconnaissance d'activités (LIARA) font fréquemment appel à des participants ayant des atteintes cognitives puisqu'il s'agit des personnes principalement visées par le développement des technologies. Dans les dernières années, des patients atteints de traumatismes craniocérébraux (TCC) ainsi que ceux souffrant de la maladie d'Alzheimer ont pris part aux recherches effectuées. Les projets de recherche dans lesquels ces participants étaient impliqués avaient pour but d'évaluer l'efficacité de technologies d'assistance cognitive auprès de ces clientèles, considérées comme étant vulnérables. Comme le recrutement et la réalisation des expériences auprès de ces populations étaient souvent complexes, il semblait nécessaire de partager avec d'autres professionnels les expériences vécues, les différents obstacles ayant été surmontés ainsi que les stratégies ayant été développées. En plus de favoriser l'approfondissement des réflexions à propos des difficultés particulières vécues par ces patients et des défis quotidiens qu'ils doivent affronter, l'objectif est également de se pencher davantage sur la place qu'occupe l'éthique dans les recherches auprès de populations vulnérables et de réfléchir à des solutions accessibles et efficaces pour faciliter les étapes du recrutement et du consentement.

\section{Présentation}

Les travaux entrepris par la Chaire de recherche sur l'intelligence ambiante et les technologies d'assistance cognitive (CRIAAC), dont fait partie le LIARA, visent le développement d’outils technologiques spécialisés subvenant aux besoins des patients avec des problématiques neurologiques. Plus particulièrement, le but de l'équipe multidisciplinaire est de permettre une plus grande autonomie dans l'accomplissement des activités de la vie quotidienne (AVQ) ${ }^{1}$ de cette population. Il est important de comprendre que les troubles vécus par les participants visés par les recherches du LIARA sont souvent envahissants et restreignent les patients dans différentes sphères de leur vie, d'où l'intérêt d'augmenter leur autonomie et de diminuer leur sentiment de détresse face à cette situation.

Deux clientèles sont principalement visées au LIARA : les personnes avec un TCC et ceux ayant l'Alzheimer. Tout d'abord, un TCC survient chez une personne ayant subi un choc important à la tête provoquant d'importantes séquelles. Selon la force d'impact de ce choc, l'importance des saignements et la présence d'un coma ou non, la gravité du TCC est établie médicalement et peut se situer de léger à sévère (Paquette, 2016). Plusieurs phases suivent l'impact et peuvent constituer des visées pour certaines recherches. En effet, certaines études débutent alors que le client est dans le coma, ce qui requiert des façons de procéder particulières pour avoir le consentement, alors que d'autres se passent plus tard dans leur réadaptation (le cas de nos études) où le patient a déjà travaillé plusieurs aspects de récupération dans des centres hospitaliers et de réadaptation. Dans les études que nous avons réalisées, il a été nécessaire de travailler avec des participants ayant subi des TCC modérés ou sévères uniquement puisque, dans ces cas, les symptômes cognitifs et comportementaux subséquents étaient plus importants. Dans le cas des patients atteints de la maladie d'Alzheimer, les patients recrutés se situaient à différents niveaux dans l'évolution de la démence, mais l'ensemble d'entre eux souffrait de troubles mné-

1. Les activités de la vie quotidiennes (AVQ) sont les activités élémentaires accomplies dans la routine habituelle d'une personne autonome et indépendante. Par exemple, cuisiner, s'habiller et se laver sont considérées comme étant des AVQ (Société Alzheimer du Canada, 2016). 
siques. Il faut également savoir que la maladie d'Alzheimer fait en sorte que les personnes atteintes présentent une importante difficulté à effectuer les activités de la vie quotidienne (AVQ) et domestique (AVD). Aussi, il est possible d'observer, chez ces personnes, une difficulté à comprendre les notions abstraites, une diminution du jugement et une perte d'initiative (Société Alzheimer du Canada, 2016).

Les prototypes de technologies à l'essai sont donc créés dans l'intention de permettre à des clientèles vulnérables d'obtenir un niveau d'indépendance supérieur en les accompagnant dans la réalisation de différentes tâches et des AVQ, comme la cuisine, ou encore d'alléger la responsabilité des proches aidants et des intervenants². Ce laboratoire, situé dans les locaux de l'Université du Québec à Chicoutimi (UQAC), est en fait un prototype grandeur nature d'appartement intelligent doté de capteurs et d'effecteurs en tout genre. Les travaux effectués sont uniques et engendrent des défis de toutes sortes, qu'il s'agisse de l'aspect psychologique, informatique, physique ou, bien entendu, de l'aspect éthique.

\section{Défis liés à l'éthique de la recherche}

L'éthique en recherche est essentielle à la protection et au respect des participants. À cet effet, des règles et des démarches bien définies se doivent d'être respectées et suivies minutieusement. Malgré la nécessité incontestable d'un tel cadre, la situation des chercheurs demeure difficile puisque les projets de recherche se voient parfois contraints à plusieurs niveaux par ces conditions. Notamment, le recrutement s'avère parsemé d'embûches lorsqu'il est question d'éthique et de consentement libre et éclairé.

\section{Article 21 du Code civil du Québec et notion de risque minimal.}

D’abord, il est nécessaire d'évaluer convenablement la notion de risque attribué à la recherche présentée. Puisqu'il était question de travailler avec une clientèle étant jugée comme vulnérable et que certains des candidats sont en situation d'inaptitude, il était nécessaire de tenir compte des directives données par la Direction de l'éthique et de la qualité du Ministère de la Santé et des Services sociaux à l'effet que «les risques encourus lors de l'expérimentation ne doivent pas être hors de proportion avec les bénéfices espérés» (EPTC2, 2014; MSSS, 2016). Ce ne fut pas problématique à l'égard des projets menés par le CRIAAC, puisque les recherches effectuées comportaient un risque minimal pour l'intégrité des participants. Par contre, les modalités liées au consentement éclairé ont provoqué quelques difficultés. En effet, dans le cadre de la l'article 21 du Code civil du Québec, un participant considéré comme inapte aux yeux de la loi ne peut consentir par lui-même à participer à l'étude (Hébert, 2014). Autrement dit, un représentant légal doit être présent pour que le recrutement soit fait adéquatement.

L'enjeu soulevé réside dans le fait que les participants susceptibles de participer aux études demeurent, la plupart du temps, dans des centres spécialisés ou dans des hôpitaux. II n’y a donc pas toujours présence de tuteurs légaux ou de personnes habilitées à consentir aux soins lors du recrutement des personnes en situation d'inaptitude ou qui sont en évaluation à ce sujet. Cet élément a contraint les membres de l'équipe du CRIAAC à augmenter leurs efforts pour faire en sorte que le personnel des centres puisse rejoindre la famille. En effet, les règles d'éthique en place et les politiques des centres empêchent les chercheurs d'entrer directement en contact avec les participants potentiels (Hébert, 2013).

2. L'une des recherches en question avait pour but de vérifier l'efficacité d'une cuisinière intelligente lors de la réalisation d'une recette de pâté chinois comparativement à l'efficacité de l'assistance humaine. Dans un premier temps, les participants étaient guidés par la cuisinière, qui émet des sons et des indications programmées préalablement, et ensuite par une personne afin de comparer la quantité d'erreurs effectuées. 
À cet effet, les intervenants sont parfois contraints à effectuer une grande partie du recrutement à la place des chercheurs et des assistants. Bien que ceux-là manifestent généralement une grande volonté d'aider, la charge de travail immense dans leur emploi les rend moins enclins à prendre en charge cette responsabilité et ils sont souvent dans l'impossibilité d'expliquer complètement le projet de recherche (qu'ils connaissent plus ou moins) ainsi que les implications qui y sont liées. Pour leur part, les médecins, qui ont la possibilité d'offrir aux patients de prendre part aux projets de recherche, mettent souvent cette option de côté puisqu'il ne s'agit pas d'un aspect prioritaire pour la santé du patient et qu'ils sont conscients que les proches sont submergés dans les démarches à effectuer.

L'entourage des patients, surtout dans les cas d'inaptitude, peut également faire preuve de surprotection. Souvent eux-mêmes en période de choc et d'adaptation, les proches tentent, parfois de façon démesurée, de protéger la personne souffrante. Ceux-ci croiront donc que les modalités et les implications liées à la recherche seront trop exigeantes et complexes pour le patient et refuseront de signer le consentement. Pourtant, les risques associés à nos recherches sont minimaux, mais la surprotection des proches demeure prédominante. Chez les médecins, il est possible d'observer ce phénomène lors de l'annonce du diagnostic. Autrement dit, ils n'osent pas toujours suggérer la participation à un projet de recherche à ce moment puisqu'ils éprouvent de la compassion et présupposent que les patients et leurs proches ne sont pas en état d'entendre parler d'une recherche.

Ces situations font en sorte qu'il y a une grande partie de la population visée à laquelle nous n'avons pas accès ou que nous devons, malheureusement, exclure de l'échantillon. Il est nécessaire de noter que les patients inaptes ou en voie de le devenir font partie de la clientèle principale visée par le développement d'outils d'assistance cognitive. En les excluant à cause de la difficulté à communiquer avec le tuteur légal ou par non-transmission de l'information relative à la recherche, il y a une perte de données d'une valeur non négligeable. Ainsi, la forte majorité des participants aptes lors des expérimentations peuvent constituer une source de biais en ce qui concerne les résultats, puisqu'ils présentent des symptômes plus légers et réussissent donc à effectuer les tâches plus facilement (Voyer et St-Jacques, 2006).

Également, en ce qui a trait au consentement éclairé, le facteur lié aux difficultés de langage des participants est central. Les patients atteints de TCC ou de la maladie d'Alzheimer souffrent souvent d'altérations de la compréhension et de la production du langage, ce qui rend plus complexes la communication et la manifestation des besoins (Proulx, 2009). Ainsi, afin d'obtenir un consentement éclairé réel, il est nécessaire de prendre le temps d'expliquer correctement la nature des implications de la participation ainsi que de s'assurer de la compréhension complète et juste de l'entièreté de ces éléments, autant pour le participant que pour le proche l'accompagnant.

\section{Le recrutement}

Outre les défis liés directement aux principes éthiques, plusieurs obstacles associés aux déficits cognitifs et aux diverses craintes des participants surviennent lors des différentes étapes d'une recherche.

Tout d'abord, il est inacceptable de tenter de recruter des participants alors qu'ils sont encore en période de choc (post-annonce du diagnostic ou post-accident). Pour ce qui est des patients atteints de TCC, leurs symptômes sont plus sévères, voire plus aigus, dans un certain délai suivant leur accident. II n'est pas rare de voir les gens atteints de TCC faire des progrès dans les mois suivants leur traumatisme. Bien que les répercussions soient nombreuses et marquantes, une récupération partielle, voire quasi totale dans de rares cas, est possible 
pour certains (Gil, 2014). Il pourrait être intéressant de les recruter durant la période suivant l'accident afin de faire l'expérimentation alors que les symptômes sont importants, mais il ne serait pas très éthique de s'inclure dans cette période intense de réadaptation et d'adaptation à la condition.

Également, le fait de ne pas pouvoir profiter des technologies étudiées dans les projets de recherche posait problème chez certains candidats approchés ou chez leurs proches. Puisque les outils conçus par l'équipe du CRIAAC ne pourront être utilisés que dans un temps indéterminé, certains mentionnaient ne pas vouloir participer par crainte de perdre leur temps ou par désintérêt des objectifs à long terme de ces projets. Ce phénomène est demeuré présent même s'il a été question de miser sur l'altruisme des participants potentiels. En effet, bien qu'un accent ait été mis sur les développements dont bénéficieront les générations futures lors des explications, le manque d'intérêt demeurait palpable puisqu'ils ne seraient pas eux-mêmes en mesure de profiter des avancés technologiques espérés. II aurait peut-être été préférable de soulever les éléments attrayants de la participation, puisqu'au final la majorité des participants qui ont accepté de prendre part aux expérimentations ont souligné leur satisfaction face à leur participation. Par exemple, certains d'entre eux ont mentionné avoir passé «du bon temps en bonne compagnie», qu'ils voyaient cela comme une «activité différente» et étaient heureux d'avoir pu parler de ce qu'ils vivaient.

Également, des craintes liées à l'utilisation des outils d'assistance cognitive étaient notables chez les patients atteints de la maladie d'Alzheimer. Ceux-ci éprouvaient beaucoup de réticence à travailler avec des outils technologiques. Puisque les gens atteints de la maladie d'Alzheimer sont souvent plus âgés, ils sont moins enclins à apprendre à travailler avec de nouveaux outils, surtout lorsqu'il s'agit du domaine technologique avec lequel ils ne sont pas familiers (Verreault, 2018).

Un autre motif qui était invoqué était le fait de ne pas pouvoir être utile en raison de leur déficit, même si la situation était tout à fait l'inverse. En raison des atteintes cognitives dont elles souffrent, ces personnes sont malheureusement parfois dans l'incapacité d'apprendre et de retenir de nouveaux éléments. Lorsqu'ils sont conscients de ce fait, les patients ont parfois la certitude de ne pas être assez compétents pour prendre part à des recherches universitaires. Ils refusent donc parfois de consentir, se sentant inutiles ou inadéquats. Pourtant, c'est principalement parce qu'ils ont ces déficits qu'ils sont intéressants aux yeux des chercheurs. Ces croyances sont évidemment erronées, mais il est fondamental de rassurer les patients à ce sujet une fois le contact établi avec eux.

\section{Contexte de réalisation des études}

Des études menées par le Centre d'études sur le stress humain (CSEH) démontrent que le fait de tester les capacités mnésiques ou cognitives des personnes âgées dans un environnement qui leur est défavorable a un impact négatif sur leur performance. Notamment, ces études révèlent que le temps entre la prise de rendez-vous et la rencontre elle-même, les modalités associées au transport et l'environnement médical peuvent créer de l'anxiété chez les personnes âgées lorsqu'il est question de tests. En effet, le CSEH démontre l'importance que joue le rôle de l'environnement dans les performances aux tests lorsqu'on compare les résultats obtenus à ceux de plus jeunes candidats (Lupien et coll., 2012).

Ces facteurs ont également été observés par l'équipe du CRIAAC. Il va de soi que la question du transport est plutôt anxiogène pour les gens dans ces situations. D'une part, s'ils sont inaptes ou qu'ils ne peuvent pas conduire, le fait de devoir trouver quelqu'un pour les accompagner ou d'organiser un transport adapté peut les angoisser et même empêcher leur consentement à participer. D'autre part, s'ils conduisent, le fait de devoir se 
rendre à l'université, de trouver un stationnement et de payer l'horodateur peut provoquer le même sentiment et, donc, une diminution de leur performance ou de leur désir de participer (Lupien et coll., 2012).

Lorsqu'on effectue des recherches dans les murs de l'université, il faut se souvenir qu'il représente un lieu jeune et dynamique dans lequel les personnes plus âgées se sentent plus ou moins à l'aise. Ainsi, ils ne seront pas attirés à participer à une étude se déroulant à cet endroit. Il est important aussi de souligner qu'il s'agit d'un lieu vaste avec plusieurs édifices et étages, ce qui peut poser problème chez les patients souffrant de la maladie d'Alzheimer puisqu'ils sont victimes d'une importante désorientation dans l'espace. Ils ont donc peur de se perdre dans les différents locaux et de ne trouver personne pour les aider à s'orienter convenablement. Lorsqu'ils ne peuvent être accompagnés par un proche pour diverses raisons, ces craintes sont accentuées. Dans la vie de tous les jours, cette désorientation constitue un important facteur d'isolement, de surveillance et de risque de fugue ou d'égarement (Ruis, 2008).

\section{Solutions \& Astuces}

Face aux nombreuses situations énumérées précédemment, l'équipe de recherche du CRIAAC n'avait guère d'autres choix que de faire preuve de créativité afin de poursuivre ses démarches. Bien qu'il ne soit pas possible d'éliminer totalement les éléments problématiques au recrutement des participants, il fut question d'utiliser différentes approches et méthodes dans le but de faciliter cette étape ou, du moins, d'en réduire les obstacles. Les caractéristiques particulières des patients ainsi que les règles strictes (mais logiques pour protéger les participants) de l'éthique en recherche ont fait en sorte que des solutions qui facilitaient à la fois le rôle des chercheurs mais également celui des participants ont été développées. En effet, plusieurs des solutions proposées permettaient aux participants d'être moins anxieux et de se sentir à l'aise en ce qui concernait le processus de consentement et amélioraient donc leur participation.

\section{Recrutement}

On ne peut passer sous silence le sujet du travail interdisciplinaire plutôt complexe qui s'impose lors d'une recherche auprès de patients vulnérables. Puisque la collaboration avec les centres et les hôpitaux est obligatoire pour le recrutement auprès de ces clientèles, le fait d'avoir une relation professionnelle de bon terme avec les intervenants est un atout. Il faut comprendre leur situation et se montrer empathique à leur égard, tout en s'adaptant à leur rythme de travail. Ainsi, ils se sentiront respectés et auront davantage envie d'aider dans le processus de recrutement. Travailler en étroite collaboration avec les intervenants et les centres permet d'approcher les participants de façon plus personnalisée, d'organiser des services mieux adaptés, d'être supporté tout au long du processus et de profiter d'une plus grande accessibilité dans le réseau. Bien souvent, les infirmières ou travailleurs du milieu connaissent leurs patients, leurs réactions, leurs inquiétudes et leurs difficultés particulières. Ainsi, travailler ensemble vers l'objectif commun d'assurer le confort et le bien-être de la personne lors de sa participation à la recherche est un aspect considérable. Il peut s'agir aussi, pour des membres de l'équipe de recherche, d'aller passer des journées dans des résidences, des centres - parfois même des fins de semaine - ou dans des événements pour avoir l'occasion de se faire connaître, d'avoir une table pour parler de la recherche où les proches peuvent venir poser des questions et voir les outils utilisés.

Il semble pertinent, lors de l'approche d'un participant, de mettre l'accent sur le but visé par la recherche et sur ses avantages. En expliquant les raisons pour lesquelles de tels projets sont mis en place et en spécifiant les bénéfices espérés, les gens ont tendance à développer de l'intérêt pour le projet en question et à s'intéres- 
ser aux avancements possibles dans le domaine. Bien souvent, les patients ont de la difficulté à s'imaginer concrètement les bénéfices des recherches effectuées et ce qu'il est possible de produire ou d'améliorer avec les résultats. Ainsi, en leur expliquant de façon précise les objectifs visés et les avantages qui en découlent, ils deviennent curieux à ce sujet et semblent plus enclins à participer aux recherches.

\section{Consentement}

Préciser les tâches à accomplir, se montrer clair et concis envers les participants quant à leurs responsabilités, souligner que leurs déficits ne sont pas problématiques pour le déroulement du projet de recherche peut faciliter l'approche vers le consentement. Précisément à cause de leurs déficits, les participants sont parfois réticents puisqu'ils sont conscients de leurs lacunes. Par exemple, les patients ayant vécu un TCC souffrent d'atteintes cognitives, c'est-à-dire que leur mémoire, leurs fonctions attentionnelles et exécutives, leur langage, leur capacité de planification ainsi que d'autres facultés peuvent être altérés (Paquette, 2016). Aussi, ils peuvent se retrouver dans l'incapacité d'apprendre de nouveaux éléments ou encore de comprendre les étapes successives nécessaires à la réalisation d'une nouvelle activité. La conception d'une recette, par exemple, peut constituer un défi de taille pour une personne ayant subi un TCC puisque les parties du cerveau nécessaires dans la planification et la rétention des diverses étapes sont généralement touchées (Bolduc, 2015).

Il est donc évident que le fait de prendre son temps et d'être patient avec eux les rassure énormément. En leur mentionnant comment l'équipe de recherche compte s'y prendre pour les aider dans les démarches liées à la participation, comme les modalités de transport et l'accueil à l'université, ils se sentiront compris et plus à l'aise. Il faut aussi tenir compte du fait que peu de gens désirent participer lorsqu'ils ne sont pas mis au courant de ce qui les attend exactement, il est donc essentiel d'avoir un discours concret et vulgarisé. Également, il va de soi que d'énumérer précisément les actions qu'ils devront effectuer lors de l'expérimentation diminue grandement les craintes et l'anxiété liées à la recherche. Une peur quant à leurs capacités physiques peut être présente due au fait que ces personnes peuvent être aussi aux prises avec difficultés motrices. Notamment, pour les patients ayant subi un TCC, l'équilibre et la coordination peuvent être affectés à différents degrés. Ceci peut évidemment causer des chutes, des blessures importantes et des diminutions d'efficacité dans la réalisation des activités quotidiennes (Dault, 1998). Il faut donc bien les renseigner sur la sécurité pour eux lors de la réalisation de l'expérimentation. Bien souvent, lorsque les actions à effectuer sont claires et bien détaillées, ils comprennent que les étapes à accomplir sont plutôt simples et qu'ils sont en mesure de les réussir contrairement à leur croyance initiale.

Afin d'avoir un consentement éclairé valide, il est nécessaire que les futurs participants comprennent bien le rôle qu'ils joueront et les implications auxquelles ils devront répondre. Ainsi, le fait d'adopter un langage familier est un atout qui facilite la création d'un lien de confiance entre le chercheur et le participant. Il va de soi qu'en adoptant un langage trop spécifique lié à un champ d'expertise le chercheur risque de perdre l'attention du participant ou encore de lui faire sentir qu'il est incapable d'accomplir la tâche demandée. Ainsi, un langage familier et plus vulgarisé peut détendre l'atmosphère et favoriser la communication. De plus, puisque les patients approchés dans le cadre des projets du CRIAAC présentent régulièrement des difficultés de langage, le fait d'adapter son discours au niveau de l'interlocuteur a un effet positif non négligeable. Également, en ce qui concerne le contact à établir avec le participant, il faut savoir tenir compte des caractéristiques comportementales liées aux atteintes neurologiques (RAPTCCQ, 2016). En effet, il est courant de voir des changements dans le comportement, des désinhibitions ou des changements d'humeur rapides. Il faut savoir écouter les participants, les encadrer avec douceur, être patient et, surtout, respecter leur rythme et entendre les émotions que 
peuvent susciter les expérimentations (autant pour les participants que pour les proches) tout en les référant au besoin à des ressources adaptées.

Selon les expériences vécues par l'équipe de recherche du CRIAAC, mettre l'accent sur le côté humain de la recherche est indispensable lors du recrutement comme tout au long du processus d'expérimentation (Wilkinson, 2002). En effet, le chercheur se doit d'être à l'écoute des besoins, des craintes et des attentes de ses participants, surtout lorsqu'il s'agit d'effectuer le recrutement et de passer à l'étape du consentement. En tant que chercheurs auprès de clientèles vulnérables, il est nécessaire de se mettre à la place des participants et de tenter de comprendre leur situation. Le fait de se questionner sur ce qui peut leur causer de l'anxiété ou les placer dans une situation inconfortable demeure essentiel, car, après tout, la recherche a pour objectif de servir les individus atteints.

L'un des éléments qui semblent avoir facilité l'intégration des participants à la recherche est l'éducation à la technologie. Puisque les technologies d'assistance cognitive sont peu connues et qu'elles engendrent de l'anxiété chez la clientèle visée, il est primordial d'en démontrer l'utilité et de permettre au participant d'être plus familier avec cette technologie avant d'entreprendre l'expérimentation. Comprendre le fonctionnement de ces machines et savoir comment les utiliser diminuera, voire éliminera, considérablement leur méfiance initiale. Aussi, il est favorable de mentionner ou de rappeler les bénéfices de l'avancement des technologies d'assistance cognitive et ce que ces prototypes pourront apporter à la vie quotidienne des gens qui y auront recours. Il ne faut pas oublier de souligner le fait qu'ils n'auront pas à gérer le fonctionnement des technologies, c'est-à-dire qu'ils ne sont pas en contrôle gadgets inconnus et de prototypes complexes. Leur tâche demeure de réaliser des activités semblables à la vie quotidienne, ce qui les rassure énormément.

Ensuite, puisque l'organisation du transport est très anxiogène chez ces patients, il est idéal de diminuer le nombre de passations. Même si, dans le cadre de certaines études, il est parfois obligatoire que les participants se déplacent à plusieurs reprises, il est préférable de réduire ce facteur. Par exemple, dans le cadre d'expérience requérant deux passations, le fait d'accorder un temps de repos allongé peut permettre à la personne de se déplacer une seule fois plutôt que deux. Cet élément peut sembler anodin, mais il peut faire une grande différence dans le recrutement. Alors que certains refuseront de participer puisqu'ils s'inquiètent des modalités de transport, les participants à qui l'alternative de diminuer le nombre de séances sera proposée seront plus enclins à consentir à participer.

D’un côté plus pratique, l'utilisation d'un système de rappel est un outil très apprécié par les participants. Puisque les clientèles visées par ces projets sont sujettes à éprouver des difficultés mnésiques et de la désorientation dans le temps (Ruis, 2008), cette astuce permet d'éviter l'oubli de rendez-vous. Par exemple, lors de la première phase expérimentale auprès des participants atteints d'Alzheimer, $25 \%$ de ces derniers ne se sont pas présentés au rendez-vous fixé puisqu'ils avaient oublié l'entente prise préalablement. Ainsi, il fut jugé pertinent de mettre en place un système de rappel par lequel il fut possible de joindre les participants, et ce, jusqu'à trois reprises. Depuis l'implantation de ce système, aucun oubli de rendez-vous n'a été rapporté par l'équipe de recherche (Lapointe, en rédaction).

Finalement, offrir une compensation aux participants pour les remercier de leur implication dans un projet peut être une source de motivation. Évidemment, les compensations monétaires semblent être les plus attirantes pour les participants. Par contre, puisque les budgets ne permettent pas toujours aux chercheurs d'offrir ce genre de récompenses, il fut question de se tourner vers une autre solution. Ainsi, un guide d'outils fut créé dans le but d'offrir une compensation accessible et utile à la clientèle (CRIAAC, 2015). Ce guide d'outil est en quelque sorte un magazine dans lequel il est possible de trouver différentes adaptations déjà existantes qui 
permettent de compenser pour plusieurs aspects de la vie quotidienne, et qui précise à quelle clientèle l'adaptation spécialisée convient de manière plus précise. Par exemple, il est possible d'y trouver des modèles de robinet à technologie DST, de plaque de cuisson à induction, d'aspirateur robot, de porte-clés siffleur, etc. De cette façon, les gens atteints d'Alzheimer ou de TCC se voient dans la capacité de trouver facilement des objets qui leur procureront une plus grande autonomie. Dans bien des cas, ce guide d'outil fut grandement apprécié.

\section{Conclusion}

À la lumière des enjeux soulevés, il est vrai de dire que la recherche auprès des populations vulnérables demeure un domaine parsemé de défis et de surprises. Bien que les règles d'éthique soient claires et préalablement définies, le passage à l'étape du recrutement et de consentement se voit complexifié par ces règles ainsi que par les restrictions qu'imposent les normes juridiques. En plus de cela, le travail auprès de clientèles vulnérables est plus difficile en raison même des problématiques particulières vécues par ces patients. Une fois la tête plongée dans le travail de recherche, il devient parfois difficile de poursuivre ses objectifs tout en respectant à la lettre chacun des principes éthiques, d'où l'insatisfaction de certains chercheurs. Toutefois, selon les expériences vécues par l'équipe du CRIAAC, en faisant preuve de créativité et en s'harmonisant aux conditions présentées, il demeure possible pour les chercheurs d'arriver à leur fin. En somme, il reste primordial de garder à l'esprit que, peu importe l'angle avec lequel ce sujet est abordé, tous tentent de travailler pour le bien des patients et pour optimiser leur condition. Que notre rôle soit celui d'assurer leur protection par l'entremise de l'éthique ou bien celui de développer les connaissances afin d'améliorer leur sort, l'essentiel demeure de conserver ces participants au centre de nos motivations et de nos efforts. 


\section{Bibliographie}

Bolduc, F. (2015). Effets du traumatisme cranio-cérébral léger chez la personne âgée sur les processus mnésiques et exécutifs, en lien avec les habitudes de vie (thèse de doctorat, Université Laval, Québec, Canada).

Chaire de recherche sur l'intelligence ambiante et les technologies d'assistance cognitive (CRIAAC). (2015). Guide d'outils: Services offerts à la population oeuvrant près d'une personne en perte d'autonomie, Repéré à http://evalorix.com/wp-content/uploads/2015/06/561-Services-offerts-population-perte-dautonomie.pdf.

Dault, M. (1998). Réduction motrice chez des sujets ayant un traumatisme cranio-cérébral (mémoire de maîtrise, Université du Québec à Trois-Rivières, Trois-Rivières, Canada).

Conseil de recherches en sciences humaines du Canada, Conseil de recherches en sciences naturelles et en génie du Canada, Instituts de recherche en santé du Canada. (2014). Énoncé de politique des trois conseils: Éthique de la recherche avec des êtres humains. (cité dans le texte comme EPTC2). Repéré à http://www.ger.ethique.gc.ca/pdf/fra/eptc2-2014/EPTC_2_FINALE_Web.pdf

Gil, R. (2014). Neuropsychologie, (6eédition). France : Elsevier-Masson.

Hébert, A-M. (2013). Modifications à l'article 21 du Code civil du Québec : enfin une bonne nouvelle pour les chercheurs et les comités d'éthique de la recherche. Consortium national sur l'intégration sociale, 05(1). Repéré à https://oraprdnt.uqtr.uquebec.ca/pls/public/gscw031?owa_no_site=4958\&owa_no_ fiche=182\&owa_bottin=

Lapointe, J. [thèse en rédaction]. Département de psychologie, Université du Québec à Chicoutimi.

Lupien, S., Sindi, S. et Wan, N. (2012). Quand on teste, est-ce qu'on stresse? Centre d'études sur le stress humain (CESH), Repéré à http://www.stresshumain.ca/stress-et-vous/stress-chez-les-aines/quand-onteste-est-ce-quon-stresse/

Ministère de la santé et des services sociaux du Québec (MSSS). (2016). Recherche portant sur des personnes mineures ou majeures inaptes, Repéré à http://ethique.msss.gouv.qc.ca/lethique-en-bref/recherche-aupres-des-personnes-mineures-ou-inaptes.html

Paquette, C. (2016). Traumatisme cranio-cérébral. Association québécoise des neuropsychologues. Repéré à https://aqnp.ca/documentation/neurologique/le-traumatisme-cranio-cerebral/

Proulx, G. (2009). «Les déficiences cognitives et démence», Société canadienne de psychologie. Repéré à http://www.cpa.ca/lapsychologiepeutvousaider/deficiencescognitivesetdemence/

Regroupement des associations de personnes traumatisées craniocérébrales du Québec (RAPTCCQ). (2016). «Le TCC, c'est quoi?», Repéré à http://www.raptccq.com/raptccq/le-tcc-cest-quoi.html

Ruis, J. (2008). Désorientation temporo-spatiale et déambulation, comment accompagner au mieux? Revue de l'infirmière, 143, 6

Société Alzheimer du Canada (2016) La maladie, 10 symptômes précurseurs. Repéré à http://www.alzheimer. $\mathrm{ca} / \mathrm{fr} /$ About-dementia/Alzheimer-s-disease/10-warning-signs

Verreault, A. (2018). Étude exploratoire sur l'attitude et les habitudes d'utilisation à l'égard de la technologie, par les personnes atteintes de la maladie d'Alzheimer, de leurs proches aidants et des intervenants travaillant auprès d'eux (thèse de doctorat, Université du Québec à Chicoutimi, Chicoutimi, Canada). 
Voyer, P. et St-Jacques, S. (2016). L'article 21 du code civil et la recherche auprès des aînés atteints de démence dans les milieux de soins de longue durée au Québec : une analyse, un constat et une proposition. Conseil des aînés du Québec, Repéré à https://www.fsi.ulaval.ca/sites/default/files/documents/pdf/Personnel/Philippe\%20Voyer/article_21_voyer_st-jacques_et_partenaires.pdf

Wilkinson, H. (2002). Including people with dementia in research. Dans Wilkinson H. (ed.) The perspectives of people with dementia: Research methods and motivations (pp. 9-25), London and Philadelphia: Jessica Kingslay Publishers. 
TITRE: «VEUX-TU PARTICIPER À MA RECHERCHE?» : PRINCIPES, ENJEUX ET STRATÉGIES CONCERNANT L'ASSENTIMENT DES ENFANTS DANS LE PROCESSUS DE RECHERCHE

Auteur(s): Isabel Côté, Renée-Pier Trottier Cyr, Kévin Lavoie et Geneviève Pagé

PUBLICATION: RECRUTEMENT ET CONSENTEMENT À LA RECHERCHE : RÉALITÉS ET DÉFIS ÉTHIQUES

PAGES: $127-145$

ISBN: 978-2-7622-0357-8

Directeurs: AnA MARIN, BÉATRICE EySERMANN ET MICHEL T. GIROUX

URI: HTTP://HDL.HANDLE.NET/11143/14112

DOI: HTTPS://DOI.ORG/10.17118/11143/14112 


\section{"Veux-tu participer à ma recherche?»: principes, enjeux et stratégies concernant l'assentiment des enfants dans le processus de recherche}

Isabel Côté, professeure agrégée, Département de travail social, Université du Québec en Outaouais

Renée-Pier Trottier Cyr, candidate à la maitrise en psychoéducation, Département de psychoéducation et de psychologie, Université du Québec en Outaouais

Kévin Lavoie, doctorant en sciences humaines appliquées, Faculté des arts et des sciences, Université de Montréal

Geneviève Pagé, professeure agrégée, Département de travail social, Université du Québec en Outaouais

Résumé : Cet article présente les considérations éthiques et les choix méthodologiques entourant l'élaboration d'une étude qualitative réalisée auprès d'enfants âgés de cinq à 13 ans au Québec. Les efforts déployés pour favoriser la participation libre et éclairée des participants sont décrits au regard des constats tirés d'une recension des écrits sur l'assentiment des enfants. Puisque ce type de recherche soulève des enjeux importants, plusieurs éléments doivent être pris en considération tels que la compréhension de l'enfant et sa capacité à assentir, la relation de pouvoir et l'équilibre des rôles entre le chercheur et le participant, ainsi que les incitatifs à la participation. Afin de favoriser l'implication des enfants dans le processus de recherche, deux outils ont été développés, soient une lettre d'information et une activité de permission. Outre une présentation de ces outils, une analyse de leur conception et de leur application est proposée afin de circonscrire les apports et les limites d'une telle démarche.

Mots-clés : enfant, éthique, assentiment, consentement, recherche qualitative. 


\section{Introduction}

Depuis quelques années, l'intérêt pour la participation des enfants dans le processus de recherche est manifeste et émane d'une préoccupation croissante envers les droits des enfants sur la scène internationale (Graham, Powell et Taylor, 2015; Razy, 2014; Willumsen, Vegar et Studsrør, 2014). L'implication des enfants dans les études portant sur des sujets qui les touchent apparaît d'autant plus essentielle que cela augmente la validité des informations recueillies auprès d'adultes, conduisant à une meilleure compréhension des réalités enfantines (Graham et Powell, 2013). Les enfants ne sont ainsi plus strictement appréhendés comme des objets passifs des recherches pouvant les concerner, mais plutôt comme des acteurs réflexifs capables de participer à la construction des savoirs (Aubrey et Dahl, 2006; Cashmore, 2011; Danic, Delalande et Rayou, 2006; Harcourt et Einarsdottir, 2011; Kirk, 2007). Ce changement paradigmatique n'est pas sans susciter certaines divergences au sein des univers savants (Christensen et Prout, 2010). Pour certains, les enfants sont perçus comme des êtres vulnérables dont la participation à la recherche doit être rigoureusement encadrée en vue d'assurer leur protection. Pour d'autres, la reconnaissance de l'agentivité des enfants et de leur droit à participer est de mise (Graham, Powell et Taylor, 2015; Kirk, 2007; Razy, 2014; Sarcinelli, 2015; Starkey et coll., 2014). Les tensions soulevées par ces deux points de vue opposés sont souvent au cœur des délibérations éthiques concernant ce type de recherche (Graham, Powell et Taylor, 2015).

Parmi ces dilemmes se trouve la question de l'obtention du consentement éclairé. Pierre angulaire du processus de recherche et de la relation unissant le chercheur aux participants, la question du consentement est un enjeu récurrent dans les écrits concernant l'éthique de la recherche auprès des enfants (Graham, et coll., 2015). Il est souvent prétendu que les enfants ne possèdent pas les compétences cognitives ou intellectuelles requises pour donner leur consentement éclairé, lequel est plutôt obtenu auprès des adultes qui en ont la responsabilité. Seul l'assentiment de l'enfant serait alors requis. Si certains réfutent cette vision réductrice de l'assentiment et considèrent que les enfants sont pleinement capables de comprendre les tenants et aboutissants de leur participation (Fargas-Malet et coll., 2010), la notion d'assentiment offre néanmoins une avenue conceptuelle appropriée pour évoquer les réalités et les besoins spécifiques des enfants.

L'assentiment évoque un sentiment de prise de pouvoir décisionnel chez l'enfant, bien que ce dernier ne soit pas toujours en mesure d'établir une entente formelle telle qu'établie par exemple par la signature d'un formulaire de consentement (Ford, Sankey et Crisp, 2007). En ce sens, l'assentiment n'est pas un concept juridique, mais réfère davantage à une sensibilité éthique visant à reconnaître l'enfant dans ses compétences, lesquelles ne doivent pas être évaluées uniquement à l'aune des attributs associés à l'âge adulte (Fargas-Malet et coll., 2010; Lambert et Glacken, 2011; Lundy et McEvoy, 2011). Cette reconnaissance varie selon le stade développemental du participant, étant donné les différentes habiletés acquises en fonction de l'âge des enfants (Meaux et Bell, 2001; Forgas-Malet et coll., 2010). En outre, même si le consentement du parent prime l'assentiment de l'enfant, l'inverse est impossible. Néanmoins, dans le cas où l'enfant refuse de participer à la recherche (dissentiment), le chercheur se doit d'accepter la réponse de l'enfant même si le parent a consenti préalablement à sa participation (Cashmore, 2011; Razy, 2014).

La question de l'assentiment chez les enfants demeure méconnue et peu documentée, notamment dans les espaces francophones. Quels sont les principes et les considérations éthiques pouvant guider les chercheurs dans une telle démarche de recherche? Cet article vise à circonscrire le concept d'assentiment en précisant ses principes éthiques et ses applications méthodologiques. Le texte est divisé en trois parties. La première partie situe l'assentiment de l'enfant au regard des savoirs scientifiques en sciences sociales et de la santé. La notion de consentement éclairé est décortiquée pour en identifier les éléments principaux. La deuxième partie 
propose une illustration concrète de stratégies mises en place pour adapter le processus de recherche conformément aux principes éthiques de l'approche centrée sur l'enfant. Outre une brève présentation de l'étude, les outils développés et les enjeux rencontrés lors de la collecte de données sont décrits et appuyés par des exemples concrets. La troisième partie propose une discussion de la pertinence de ces outils, ainsi que leurs apports et leurs limites en recherche qualitative.

\section{L'enfant et l'assentiment : constats tirés des écrits}

L'émergence de l'analyse théorique de la «nouvelle sociologie de l'enfance» (Hodson, 2012; Mason et Hood, 2011) de même que l'identification des enfants en tant que groupe minoritaire (Convention des Nations Unies relative aux droits de l'enfant, 1989) ont contribué à la reconnaissance des enfants comme agents sociaux au sein de la recherche et au déploiement de méthodologies adaptées afin de favoriser leur participation pleine et entière (Razy, 2014).

Assentir à une recherche ne concerne pas uniquement les considérations liées à la participation; à travers cette expérience, l'enfant fait aussi l'apprentissage d'une forme de citoyenneté (Montero, 2009). De fait, offrir aux enfants un rôle actif dans la prise de décision favorise leur évolution en tant qu'individus autonomes et leur pratique d'autogouvernance (Ford, Sankey et Crisp, 2007). En les incluant à part entière dans la recherche, Ford et ses collègues (2007) estiment que les enfants ont l'opportunité de prendre conscience et d'expérimenter le respect d'autrui à leur égard. Plusieurs considérations éthiques doivent être exposées afin de mieux cerner les défis auxquels sont confrontés les chercheurs afin de s'assurer que les enfants participant aux recherches soient en mesure de bien saisir les tenants et aboutissants de leur assentiment. À cet effet, différents éléments-clés peuvent interférer, soient la compréhension qu'a l'enfant de sa participation à la recherche, la capacité avérée à assentir, la relation de pouvoir entre le chercheur et l'enfant et, finalement, les incitatifs à la participation (Kirk, 2007; Gallagher, 2009; Graham et coll., 2013). Chaque élément renvoie à un assortiment d'attitudes et de pratiques.

\section{Adapter les outils pour favoriser la compréhension de l'enfant}

Les informations transmises préalablement à la participation à une recherche doivent permettre au participant d'en comprendre les tenants et aboutissants. De nombreux auteurs soulignent l'importance de développer des outils permettant de transmettre aux enfants des informations sur la nature de leur participation qui soient adaptées à leurs réalités (Bray, 2007; Diekema, 2006; Lambert et Glacken, 2011; Meaux et Bell, 2001; Miller, Drotar et Kodish, 2004).

Les informations à transmettre à l'enfant pour s'assurer de son assentiment sont très similaires à celles expliquées à un participant adulte : la présentation de l'équipe de recherche, le projet, les avantages et les inconvénients de la recherche, ses droits, les paramètres de confidentialité, son droit de se retirer en tout temps de la recherche et les précisions concernant l'enregistrement audio et vidéo (Graham et coll., 2013; Ford, Sankey et Crisp, 2007; Twycross, Gibson et Coad, 2008). Plusieurs moyens peuvent être utilisés pour aider à la compréhension de l'enfant : des informations audiovisuelles, écrites, visuelles, voire assistées par ordinateur (Bray, 2007; Graham et coll., 2013).

Le contenu des informations transmises, qu'il s'agisse de mots ou d'images, doit être en adéquation avec le développement cognitif de l'enfant, de ses compétences de même que son contexte socioculturel d'origine afin de s'assurer de sa compréhension (Ford, Sankey et Crisp, 2007; Graham et coll., 2013; Lambert et Glacken, 2011). 
Outre la conclusion selon laquelle la compréhension dont les enfants font preuve augmenterait avec l'âge, Miller et ses collaborateurs (2004) nous renseignent sur les dimensions de l'assentiment les mieux comprises par les enfants ainsi que celles qui semblent plus difficiles à saisir pour eux. Parmi celles faciles à comprendre pour les jeunes participants, notons les façons de mettre fin à la participation, l'assurance qu'aucunes représailles ne seront opposées à un refus de participer, le caractère volontaire de la participation et la liberté de poser des questions aux chercheurs. Les dimensions moins bien intégrées concernent les risques, avantages et inconvénients liés à la participation (Miller, Drotar et Kodish, 2004) - bien que la compréhension de ces éléments soit surtout tributaire de la façon dont l'information est présentée à l'enfant (Burke, Abramovitch et Zlotkin, 2005).

La qualité des outils utilisés afin de transmettre des informations adaptées à la compréhension des enfants revêt une importance certaine. Ainsi, de jeunes enfants ou encore des enfants ayant des difficultés d'apprentissage sont en mesure de comprendre les informations qui leur sont présentées et de prendre des décisions éclairées en regard de leur participation, du moment que le médium utilisé et les informations transmises sont en adéquation avec leur développement (Powell et coll., 2012). Dans tous les cas, le chercheur doit s'assurer que les mots utilisés sont compris de façon univoque par l'enfant (Gallagher, 2009), puisque les erreurs d'interprétation ne sont pas toujours détectées (Gallagher et coll., 2010). Emond (2006) propose ainsi de demander à l'enfant ce qu'il a compris de la recherche à partir des informations préalablement obtenues par ses parents ou par un dépliant explicatif, à la suite de quoi les buts, objectifs et processus de recherche seront clarifiés. Cette procédure permettrait de respecter davantage le rythme de l'enfant et de s'assurer d'un assentiment éclairé de sa part (Emond, 2006).

\section{Évaluer la capacité de l'enfant à assentir}

Le deuxième élément-clé suppose que le participant ait les compétences requises pour comprendre la nature du consentement (Kirk, 2007). Si les différents comités éthiques considèrent l'âge comme critère déterminant, de nombreux chercheurs contestent cette vision déterministe susceptible d'oblitérer d'autres aspects permettant d'évaluer les capacités de compréhension qu'a l'enfant du processus de recherche, par exemple son niveau de maturité, les connaissances qu'il possède et le contexte social dans lequel s'insère la recherche (Gallagher, 2009; Graham, Powell et Taylor, 2015; Parizeau, 2015; Razy, 2014; Sarcelli, 2015). Il y a une hétérogénéité des opinions quant à l'âge auquel un enfant peut assentir à sa participation. Pour certains, l'enfant aurait les compétences nécessaires dès le début de l'âge scolaire (Broome, 1999; Ford, Sankey et Crisp, 2007) alors que d'autres soutiennent que l'assentiment est intelligible seulement à partir de sept ans (Diekema, 2006), onze ans (Tait et coll., 2003) ou même treize ans (Fundudis, 2003). Enfin, une dernière catégorie de chercheurs considère que la capacité d'assentiment est située sur un continuum où l'information transmise se précise en fonction de l'âge des enfants (Miller et Nelson, 2006).

Dans les pays anglo-saxons, le principe de Gillick est fréquemment utilisé pour évaluer la capacité des enfants à consentir. Appliqué à l'origine à la capacité d'un mineur de moins de 16 ans d'avoir préséance sur le détenteur de l'autorité parentale quant aux décisions de nature médicale le concernant, le principe de Gillick a par la suite peu à peu investi le champ des recherches en sciences sociales (Gallagher, 2009; Powell et coll., 2012). Comme l'expliquent Marrow et Ricards (1996, dans Powell et coll., 2013 : 14, traduction libre), ce principe stipule qu'un enfant compétent est «celui qui atteint une compréhension et une intelligence suffisante pour lui permettre de bien comprendre ce qui est proposé». Ces auteurs ajoutent que l'enfant compétent détient un «pouvoir discrétionnaire pour lui permettre de faire des choix judicieux dans son propre intérêt». 


\section{Être conscient de la relation de pouvoir entre le chercheur et l'enfant}

Le troisième élément-clé dans l'obtention de l'assentiment de l'enfant concerne la nature des rapports hiérarchisés entre les enfants et les adultes et la position d'autorité qui en découle. Cela peut faire en sorte que les enfants se sentent contraints de coopérer lors d'un appel à participation (Gallagher et coll., 2010; Graham et coll., 2013). En ce sens, les chercheurs intervenant dans des contextes valorisant l'obéissance des enfants, tels les milieux scolaires et médicaux par exemple, doivent être particulièrement vigilants à ce que cela ne se produise pas lors de la mise en œuvre du projet de recherche. Dans ces deux contextes, les enfants tendent à respecter les demandes et exigences des adultes et sont donc susceptibles de reproduire ce modèle relationnel basé sur la soumission à l'autorité (Alderson et Morrow, 2011). Pour Lambert et Glacken (2011), il y a alors lieu de se questionner sur l'autonomie réelle de l'enfant et sur sa liberté à participer de façon volontaire à la recherche.

Une autre manifestation des rapports hiérarchisés pouvant contraindre l'assentiment se manifeste par le statut juridique des enfants qui les subordonne aux adultes qui en ont la responsabilité, soient leurs parents ou tuteurs légaux. Bien que la plupart des chercheurs jugent que le consentement des détenteurs de l'autorité parentale est non seulement souhaitable, mais éthiquement nécessaire (Graham et coll., 2013; Razy, 2014), d'autres soutiennent que cela peut poser problème, notamment dans des contextes litigieux nécessitant la confidentialité pour protéger les participants. Ceci serait particulièrement le cas de recherches portant sur les jeunes marginalisés, ou encore les situations de violence intrafamiliale (Alderson et Morrow, 2011; Carroll-Lind et coll., 2006; Powell et coll., 2012). Dans les recherches portant sur la maltraitance des enfants, les parents ou les tuteurs légaux pourraient refuser leur consentement afin de protéger la cellule familiale et empêcher les enfants de révéler les problèmes qui y ont cours (Cashmore, 2011). Certains chercheurs, à l'instar de Caroll-Lind et ses collègues (2006), privilégient alors le consentement passif des parents ${ }^{1}$, c'est-à-dire leur obligation de faire valoir expressément leur non-consentement, bien que cette procédure soit controversée et habituellement sujette à caution de la part des comités d'éthique (Graham et coll., 2013; Powell et coll., 2012).

Les enfants peuvent avoir de la difficulté à opposer un refus verbal au chercheur en cours de protocole par crainte d'impolitesse (Alderson \& Morrow, 2011; Ducan et coll., 2009; Gallagher, 2009). C'est pourquoi les chercheurs doivent être en mesure de bien évaluer les signes comportementaux de dissentiment (tels la passivité, le manque de coopération, le silence, l'irritabilité, les signes d'ennui, etc.) afin de les interpréter pour ce qu'ils sont, c'est-à-dire un retrait de l'assentiment (Gillies et Robinson, 2012; Graham et coll., 2013).

\section{Les incitatifs à la participation}

Le fait d'offrir un incitatif, sous la forme d'une récompense ou d'un dédommagement, ne fait pas consensus dans les écrits scientifiques. Certains considèrent que cela valorise la participation de l'enfant (Bushin, 2007; Gallagher, 2009), alors que d'autres estiment plutôt que cela peut agir comme motivation extrinsèque et, conséquemment, biaiser le caractère libre et volontaire de l'assentiment (Fargas-Malet, et coll., 2010). Si le fait d'offrir une récompense est jugé plus éthiquement acceptable dans le cadre de recherche à risque faible ou négligeable (ne suscitant que de l'inconfort), cela est très critiqué dans les recherches à risque élevé puisque cela peut conduire les enfants à choisir d'accepter des conséquences plus importantes qu'ils ne l'auraient fait sinon. (Graham et coll., 2013; Rice et Broome, 2004). Les recherches conduites auprès d'enfants issus de milieux défavorisés illustrent également les tensions existant dans les écrits concernant l'incitation à la participation,

1. En général, une non-réponse de la part du détenteur de l'autorité parentale est considérée comme un refus. Dans le cas du consentement passif, en l'absence de refus formel, le détenteur de l'autorité parentale sera réputé avoir donné son consentement. 
puisqu'il est plus facilement admis que le dédommagement peut avoir un impact globalement positif sur l'enfant (Graham et coll., 2013).

II n'existe pas, à l'heure actuelle, de lignes directrices concernant la valeur acceptable du dédommagement (Bushin, 2007; Fargas-Malet et coll., 2010; Rice et Broome, 2004). Il incombe donc au chercheur de trouver la juste valeur de l'incitation, laquelle varie considérablement selon les recherches (Rice et Broome, 2004). À ce propos, Lépine et ses collègues (2011) proposent des compensations symboliques pour les enfants de moins de 12 ans, sous forme de cadeaux à faible valeur monétaire, et des cartes-cadeaux pour les adolescents. Rice et Broome (2004) suggèrent, quant à eux, que les chercheurs œuvrant auprès des enfants se questionnent sur la remise éventuelle d'une récompense. D’abord, ils doivent explorer le type de récompense remise dans des études similaires. Ensuite, se questionner quant aux attentes des enfants de recevoir ou non une récompense. Les récompenses doivent également être en adéquation avec le degré de développement des participants. Lors du choix de la récompense, les chercheurs doivent faire preuve de respect, de bienveillance et d'équité.

\section{Obtenir l'assentiment de l'enfant : une illustration concrète}

La prochaine section présente les stratégies méthodologiques déployées pour favoriser l'implication des enfants dans la recherche et pour aménager un dialogue leur permettant d'accepter ou non de participer. Nous avons divisé cette section en cinq parties afin de retracer notre démarche. D’abord, nous décrivons sommairement l'étude dans laquelle s'inscrivent nos réflexions sur l'assentiment. Ensuite, nous expliquons la logique qui a sous-tendu le développement des outils utilisés dans le cadre de cette recherche pour obtenir l'assentiment de nos jeunes participants. Enfin, par notre illustration, nous proposons une application concrète des enjeux développés dans la recension des écrits, soient l'âge de compréhension, les enjeux de pouvoir et l'incitatif à la participation. En conclusion, nous procéderons à l'analyse réflexive portant sur l'utilité des outils que nous avons développés.

\subsubsection{Présentation de l'étude}

Les enjeux décrits dans ce texte découlent de nos réflexions qui émergent d'une recherche en cours sur les constellations familiales et relationnelles d'enfants issus de familles homoparentales et adoptives au Québec. Subventionné par le Conseil de recherche en sciences humaines du Canada, ce projet de recherche vise à documenter les représentations des liens biologiques et sociaux des enfants et leurs impacts sur leur conception de la famille. Le but de l'étude est de mieux cerner le processus de réflexion des enfants les menant à identifier les personnes qui composent leur famille et leur entourage, et la nature des liens qui les unissent. Elle s'appuie sur trois études réalisées précédemment par les chercheures de l'équipe auprès des parents de ces enfants, soient deux recherches menées auprès de mères lesbiennes et de pères gais ayant fondé leur famille à l'aide de tiers reproducteurs, et une autre consacrée à l'expérience de parents ayant accueilli leur enfant par le biais du programme Banque mixte des centres jeunesse. Nous avons ainsi rencontré vingt-six enfants dans cinq régions du Québec (Montréal et ses environs, Outaouais, Laurentides, Estrie, Bas-du-Fleuve), soient 12 filles et 14 garçons âgés de quatre à 13 ans. 
Notre étude a été construite à partir d'une méthodologie novatrice composée de deux modes de cueillette de données, soient la réalisation avec l'enfant d'une cartographie circulaire qui illustre sa compréhension des liens avec les membres de son entourage puis un entretien semi-dirigé visant à situer la trame narrative de son histoire familiale. L'approche centrée sur l'enfant (child centered research) constituant notre appui théorique (Lambert et Glacken, 2011; Mason et Hood, 2011), nous avons voulu laisser une grande place aux enfants dans notre processus de recherche, notamment quant à l'assentiment à participer.

\section{Des exemples d'outils pour favoriser la compréhension de l'enfant en fonction de son groupe d'âge}

Peu d'outils existent actuellement pour prendre en compte le caractère unique et la spécificité de l'enfance dans le processus d'assentiment à participer à une recherche, ce qui nous a donc amenés à concevoir nos propres outils, soient la lettre d'information et l'activité de permission. Nous les décrivons un peu plus loin.

Dans le cadre de notre étude, nous avons opté pour des outils alliant la lecture de courts énoncés et la reconnaissance d'images, puisqu'il est reconnu que la communication peut ainsi être facilitée, ce qui a comme résultante de favoriser la participation active de l'enfant (Lewis et Porter, 2004). L'activité visuelle permet également à l'enfant de mieux comprendre les informations qui lui sont données quant à la nature de sa participation (Bray, 2007).

Considérant l'importance d'accorder à l'enfant le temps de réfléchir à sa décision de participer ou non (Lambert et Glacken, 2011) et la distance géographique qui nous séparait de la plupart des enfants, nous avons décidé de procéder, comme le suggèrent Ford et ses collègues (2007), en deux étapes. Dans un premier temps, nous avons envoyé par la poste une lettre d'information personnalisée pour chaque enfant que nous souhaitions rencontrer. Dans un deuxième temps, nous avons procédé à une activité de permission, animée la journée même de l'entrevue, afin de s'assurer de l'assentiment libre et éclairé de l'enfant. Ces deux outils sont présentés dans les prochaines sections.

\section{La lettre d'information}

La lettre est un moyen d'offrir aux enfants les informations nécessaires qui se retrouvent sur un formulaire d'assentiment. En lui envoyant la lettre par la poste au moins une semaine avant l'entrevue, cela permet à l'enfant de prendre connaissance de certaines notions de la recherche, de poser des questions à ses parents, ainsi que d'exprimer son dissentiment, le cas échéant. En employant cette procédure, nous évitons également de confronter l'enfant à une prise de décision sur le vif et en face à face, au cours de laquelle il pourrait avoir de la difficulté à exprimer ses besoins ou ses réticences (Bray, 2007). De plus, la lettre est un moyen de «mise en interaction» (Renou, 2005), puisqu'il s'agit du premier contact entre l'enfant et les membres de l'équipe de recherche, et que ces lettres présentent la personne appelée à conduire l'entrevue auprès de l'enfant (Figure 1). L'enfant est donc en mesure de nous reconnaître lorsqu'il nous rencontre. Cela permet aussi de briser la glace, d'établir un lien de confiance et de faciliter le déroulement de l'entrevue. Plusieurs enfants nous ont d'ailleurs confié se sentir à l'aise de nous rencontrer puisque, grâce à notre photo sur la lettre, ils avaient l'impression «de nous connaître déjà un peu».

Nos modèles de lettres d'information ont été construits en vertu des recommandations formulées par Ford et ses collègues (2007), c'est-à-dire en privilégiant des images qui permettent à l'enfant de saisir le sens des informations qui lui sont présentées, de même que le recours à un langage simplifié et univoque. 
Nous avons décidé de diviser les enfants en quatre groupes d'âge en fonction des cycles scolaires et de créer autant de modèles de lettres d'information : les enfants de maternelle et ceux du premier cycle (5-7 ans), ceux du deuxième cycle (8-9 ans), ceux du troisième cycle (10-11 ans) ainsi que les jeunes qui fréquentent l'école secondaire (12-13 ans). Chaque lettre était adressée directement à l'enfant concerné, et, dans le cas de fratrie, chaque enfant recevait sa propre lettre adaptée à son âge. Les figures 1 et 2 présentent le modèle de lettre conçu pour les plus jeunes enfants, soient ceux âgés de cinq à sept ans.

Figure 1. Modèle (recto) de lettre d'information pour les enfants âgés de 5 et 6 ans
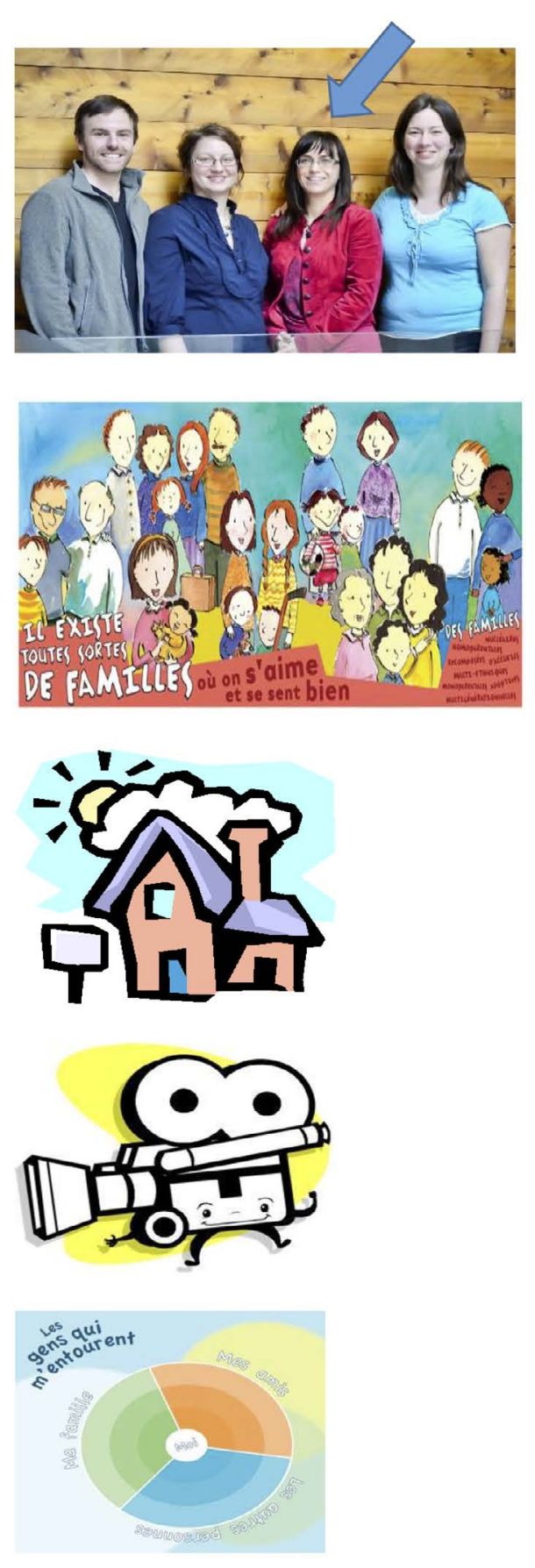

\section{Lettre d'informations}

Bonjour ! Mon nom est Isabel et je suis professeure et chercheure à l'université. Je travaille aussi avec Geneviève, Kévin et Renée-Pier. Lors de mes rencontres, je suis toujours accompagnée d'un membre de mon équipe.

J'aimerais parler avec toi de ta famille et des gens qui font partie de ta vie. J'aimerais te parler spécialement à toi parce que je pense que tu peux m'apprendre des choses.

Si tu dis oui pour me rencontrer, je viendrai chez toi pendant environ une heure.

J'amènerai une caméra vidéo pour filmer notre rencontre pour éviter que j'oublie des petites choses.

Nous allons parler de ce que c'est, pour toi, une famille et nous ferons une activité. Mon rôle sera de t'écouter et de bien comprendre ce que tu dis. 

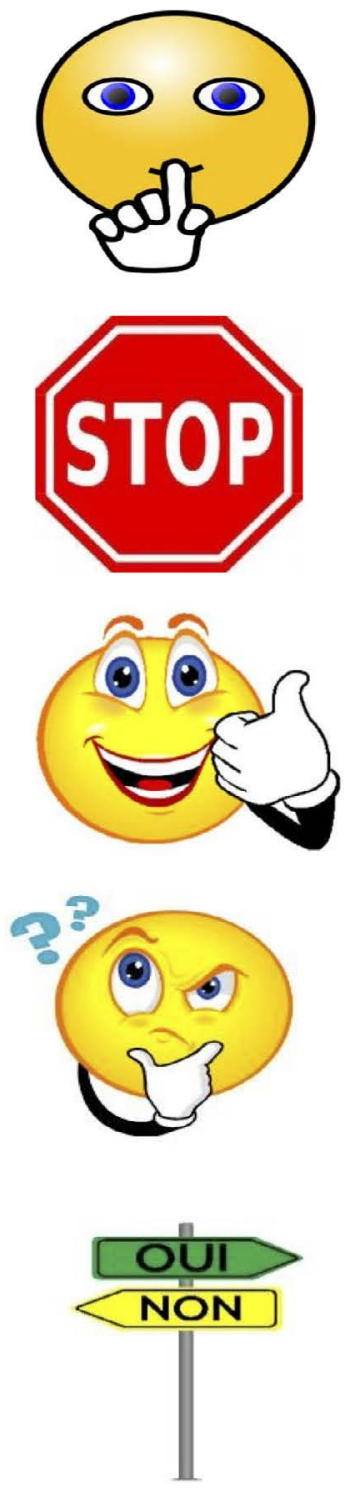

Moi, je vais garder secret tout ce que tu me dis aujourd'hui. Toi, tu peux choisir de dire ce que nous avons fait ensemble à qui tu veux.

Si tu te sens fatigué, tu peux prendre une pause. Tu peux aussi décider de ne pas répondre aux questions ou arrêter de faire l'activité.

En participant à mon projet, tu m'aides à comprendre ce qu'est une famille pour un enfant de ton âge.

Tu peux poser les questions que tu veux au moment que tu veux et j'y répondrai avec plaisir.

C'est à toi de dire si tu veux me rencontrer ! Ne t'inquiète pas, personne ne sera fâché si tu dis non!

Si tu dis oui, je vais te demander de signer une feuille qui nous donne la permission de venir te rencontrer.

\begin{tabular}{|c||}
\hline Informations sur la chercheure principale \\
Isabel Côté, Ph.D. \\
Professeure, Département de travail social \\
Université du Québec en Outaouais \\
Pavillon Alexandre-Taché \\
819-595-3900 poste 2334 \\
Isabel.cote@uqo.ca \\
\hline
\end{tabular}


Comme pour la lettre d'information, le format de l'activité de permission diffère selon l'âge de l'enfant afin de s'adapter à son niveau de développement cognitif. Pour les enfants âgés de quatre à huit ans, nous avons eu recours à une activité interactive (figure 3). Elle a été élaborée à partir des neuf concepts et images qui se retrouvent dans la lettre d'information. Les images correspondant à un concept sont placées de façon aléatoire à droite des énoncés. La consigne est simple : l'enfant doit relier par une flèche les affirmations aux images correspondantes. L'enfant est alors avisé que toutes les réponses proposées sont acceptées, «puisque, dans cette activité, il n'y a pas de mauvaise réponse», le but de l'exercice étant bien entendu de valider sa compréhension des informations et son désir de participer à la recherche.

Pour les enfants de neuf ans et plus, nous avons décidé que la lettre de permission (figure 4) leur serait adressée directement. Notre expérience clinique en travail social et en psychoéducation nous incite à croire qu'il est possible de discuter des concepts reliés à l'assentiment pour ces enfants sous forme de conversation. Pour cette tranche d'âge, l'assentiment ne transige plus par une activité interactive, mais simplement par la lecture de la lettre de permission avec l'enfant puisque ce dernier possède la maturité cognitive ainsi que des habiletés en lecture adéquate pour procéder de cette façon. La lettre est alors utilisée comme moyen de mise en relation avec l'enfant. Au regard du déroulement des entrevues réalisées jusqu'à maintenant, nous estimons qu'elle procure un espace adéquat et suffisant pour entamer le dialogue sur les considérations éthiques de la recherche.

Figure 3. Modèle de l'activité de permission pour les enfants âgés de 4 à 8 ans.

Aujourd'hui, nous ferons une activité ensemble.

Moi, je vais garder secret tout ce que tu me dis aujourd'hui. Toi, tu peux choisir de dire ce que nous avons fait ensemble à qui tu veux.

II n'y a pas de bonnes ni de mauvaises réponses. C'est ton opinion à toi qui compte.

J'ai amené une caméra vidéo pour filmer notre rencontre afin d'éviter que j'oublie des petites choses.

Mon rôle est de t'écouter et de bien comprendre ce que tu dis.

Si tu te sens fatigué, tu peux prendre une pause. Tu peux aussi décider de ne pas répondre à des questions ou d'arrêter de faire l'activité.
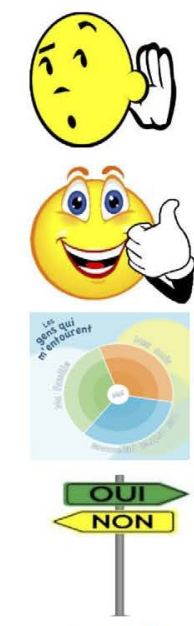

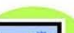

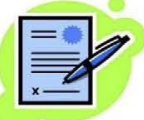

C'est à toi de décider si tu veux faire l'activité ! Ne t'inquiète pas, si tu dis non, personne ne sera fâché!

Tu peux poser toutes les questions que tu veux.

Si tu dis oui, et me donnes la permission de faire l'activité avec toi, je vais te demander d'écrire ton nom sur la feuille. Moi aussi j'écrirai mon nom.

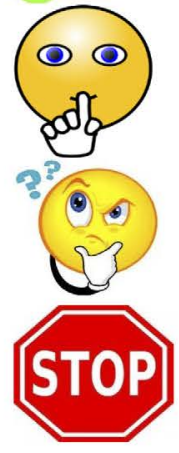


Figure 4. Modèle de lettre de permission pour les enfants âgés de 9 ans et plus.

Récits d'enfants sur leur famille

\section{Lettre de permission}

\section{Aimerais-tu participer à ma recherche?}

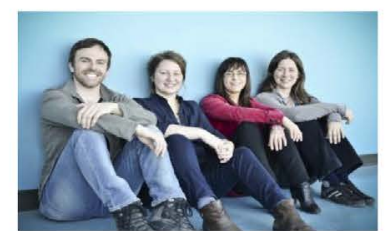

Salut! Nous sommes une équipe de recherche de l'universite qui s' Interesse a la taçon dont les jeunes se représentent leur famille. Nous pensons donc que tu es le mieux placé pour nous apprendre des choses à ce sujet. Nous aimerions donc te parler de ta famille et des gens qui font partie de ta vie.

\section{Qu'est-ce que la confidentialité?}

Tu seras filmé pendant la rencontre. Tout ce que tu feras restera entre nous. Mais toi, tu peux décider d'en parler à qui tu veux. Seulement l'équipe de recherche pourra avoir accès à ce que tu auras dit et fait. Tout le matériel sera conservé dans un classeur barré.

\section{Qu'est-ce qui pourrait te déranger pendant la rencontre?}

Tu pourrais ne pas vouloir répondre à certaines questions et tu n'y es pas obligé. À tout moment, tu peux arrêter notre rencontre. Si tu te sens fatigué, nous pouvons aussi prendre une pause.

\section{Qu'est-ce qui pourrait être positif pendant la rencontre?}

En participant à la rencontre, tu as ta place pour dire ton opinion. C'est nous qui devons t'écouter parce que nous pensons que c'est toi l'expert de la situation.

\section{As-tu des questions?}

Tu peux poser toutes les questions que tu veux, maintenant ou plus tard, et j'y répondrai avec plaisir.

Et puis, veux-tu participer à ma recherche?

C'est à TOI de dire oui ou non. Tu es complètement libre de choisir ! Si tu dis non, personne ne sera fâché ni déçu. Tout le monde respectera ta décision. Si tu dis oui, tu peux aussi changer d'idée et arrêter la rencontre en tout temps!

Ta signature :

Date :

Nos signatures :

Date :

Date :

\begin{tabular}{|c|}
\hline Informations sur la chercheure principale \\
Isabel Côté, Ph.D. \\
Professeure, Département de travail social \\
Université du Québec en Outaouais, Pavillon Alexandre-Taché \\
819-595-3900 poste 2334 \\
isabel.cote@uqo.ca \\
\hline
\end{tabular}

\section{Expérimentation des outils et ajustements}

Les outils présentés ont fait l'objet d'un prétest auprès de onze enfants âgés de cinq à treize ans (quatre garçons et sept filles) afin d'en évaluer leur pertinence. Plusieurs modifications ont été faites suite à ces prétests pour aboutir à la version définitive. La première mouture de l'activité de permission pour les plus petits était, au départ, fort différente de celle présentée. 
D’abord, l'activité était trop longue pour la capacité d'attention des enfants, principalement des plus jeunes, ce qui avait un impact considérable sur leur intérêt à poursuivre l'activité de recherche proprement dite. Ensuite, certaines images et explications associées étaient comprises de façon équivoque par certains enfants, causant une source d'inconfort. La notion de confidentialité et l'image qui y était associée au départ se sont avérées particulièrement difficiles à comprendre. Nous avions alors suivi l'avis de Bray (2007) stipulant que l'image d'un cadenas pour exemplifier la confidentialité est plus appropriée que le pictogramme représentant un visage avec un doigt sur la bouche (signe de silence). En effet, pour l'auteure, une telle image pourrait créer un malaise chez l'enfant, le mettant dans un possible conflit de loyauté s'il voulait parler de la rencontre avec autrui. Néanmoins, l'image du cadenas s'est révélée non significative pour la plupart des enfants, et nous avons donc opté pour le pictogramme dans la version définitive.

Par ailleurs, notre explication de la confidentialité a été comprise, lors des prétests, comme une injonction au silence par certains enfants. Ainsi, suite à sa participation, une fillette de neuf ans s'est exclamée à sa mère qui l'attendait hors du local d'expérimentation : «Maman, j'ai beaucoup aimé ce que j'ai fait et je ne peux pas rien te dire». Puis, se tournant vers les chercheures, de se demander à haute voix si c'était grave si un jour elle «s'échappait». Soucieux de ne pas vouloir induire chez les enfants de tiraillement entre le désir de s'exprimer à propos de la recherche et la parole donnée aux chercheurs, nous avons changé la formulation de notre explication en situant que l'obligation de confidentialité n'appartenait qu'à l'équipe de recherche seulement: «C'est nous, les chercheures, qui devons garder le silence sur tout ce que tu diras pendant la rencontre. Mais toi, tu es libre d'en parler à la personne de ton choix, si tu en as envie».

Enfin, c'est suite aux prétests que nous avons décidé d'élaborer la lettre d'information. Lors des prétests, les objectifs de la participation des enfants étaient expliqués aux parents qui se voyaient déléguer la responsabilité d'informer leur enfant. Cette procédure a entrainé plusieurs mésinterprétations et quiproquos, les enfants ne comprenant pas très bien pourquoi nous les sollicitions. La lettre d'information a donc permis de corriger la situation tout en ayant le mérite d'assurer à l'équipe de recherche que l'information transmise serait la même pour l'ensemble des participants.

\section{Déterminer l'âge de compréhension de l'enfant}

Nous avons observé lors de la passation des entrevues qu'il est généralement plus facile pour les enfants fréquentant l'école de comprendre les notions liées à l'assentiment. Étant donné la nature de l'activité de permission proposant un exercice similaire à ceux proposés en classe par les enseignants, les enfants scolarisés captaient rapidement le déroulement de la rencontre et les consignes.

Le tempérament de l'enfant influence également sa compréhension de l'activité de permission proposée. Nous avons observé qu'un enfant extraverti a une plus grande aisance à partager son point de vue, à poser des questions ou à confirmer sa compréhension des notions expliquées. L'enfant timide, quant à lui, présente davantage de réponses courtes et évasives, nous obligeant à reformuler de différentes façons les énoncés pour nous assurer de sa compréhension.

Enfin, nous sommes restés sensibles aux signes non verbaux de dissentiment pouvant être manifestés par un enfant lors des entrevues. Tel que précisé dans la recension des écrits, un enfant peut avoir de la difficulté à exprimer verbalement son dissentiment de peur de paraitre impoli ou par crainte de décevoir. Nous avons alors interprété tout comportement d'agitation, d'inattention ou d'inconfort comme un retrait de l'assentiment. Ce fut le cas pour trois enfants rencontrés : le premier, âgé de cinq ans, digressait de la conversation, 
la seconde, âgée de six ans, manifestait une timidité importante alors que le troisième, âgé de neuf ans, mais présentant des limites cognitives, a explicitement refusé de répondre à plusieurs questions, entraînant ainsi la fin de l'entrevue.

\section{Favoriser l'équilibre des rôles entre le chercheur et l'enfant}

Dans le cadre de notre recherche, nous avons mis en place trois moyens pour favoriser l'équilibre des rôles entre le chercheur et l'enfant et ainsi permettre une répartition du pouvoir entre les protagonistes. D'abord, nous nous sommes assurés, dès l'activité de permission, que l'enfant était en mesure de mettre fin à l'entrevue s'il le désirait. Nous lui avons expliqué qu'il peut dire «stop» ou simplement sortir de la pièce s'il en ressent le besoin, en précisant que c'est son droit en tant que participant. Nous avons évalué son niveau de confort à mettre en pratique ces techniques, le cas échéant.

Ensuite, nous avons fait primer le dissentiment des enfants sur le consentement parental. Nous avons ainsi appuyé une fillette lors de l'expression de son désaccord à participer à la recherche, et ce, malgré les incitations émises par ses parents et sa sœur.

Enfin, selon l'étude de Spyrou (2011), la posture physique qu'adopte le chercheur qui mène l'entrevue peut influencer les réponses de l'enfant. Par conséquent, nous avons décidé de nous ajuster à la manière dont l'enfant désirait que l'entrevue se déroule, que ce soit sur une table ou directement sur le sol. Outre la prise de pouvoir de l'enfant sur le processus de recherche, cette attitude décontractée et complice nous a permis d'instaurer un climat de confiance en amenuisant la position hiérarchique du chercheur.

\section{Souligner la participation de l'enfant : la récompense}

Nous avons décidé d'offrir une récompense aux enfants pour les remercier de leur collaboration. Comme il importe de respecter le caractère libre et volontaire de l'assentiment, nous avons toutefois opté pour une procédure particulière. D’abord, la récompense, d'une valeur approximative de dix dollars, n'était pas mentionnée dans les lettres d'information, dans les activités de permission ou lors des entrevues afin que celle-ci n'agisse pas comme motivateur extrinsèque et donc fausse le caractère libre et volontaire de l'assentiment. Après l'entrevue (ou lorsqu'un enfant mettait fin à la rencontre), nous rassemblions les enfants de la famille en les remerciant chaleureusement de leur participation. C'est à ce moment que nous leur offrions de piger dans notre «sac à surprises». Nous avons également pris soin de ne pas exclure les membres d'une fratrie trop jeunes pour la passation de l'entrevue ni ceux ayant exprimé leur dissentiment à participer en leur permettant eux aussi de se choisir une surprise. Un vaste éventail de cadeaux ont été offerts, des crayons de couleur jusqu'aux cartes-cadeaux en passant par des jeux de construction, et ce, afin que chaque enfant, peu importent son âge ou ses centres d'intérêt, y trouve son compte.

\section{Analyse rétrospective de notre démarche de recherche}

Plusieurs études reconnaissent la valeur de l'utilisation de méthodes originales et novatrices lors de recherches portant sur des populations négligées ou, du moins, peu étudiées, comme c'est le cas des enfants (Fernandez, 2003; Hill et coll., 2004). Selon ce point de vue, les méthodologies adaptées permettent de se distancer des termes utilisés par les adultes à l'égard des objets étudiés pour reconnaître l'enfant dans sa singularité, ses compétences et ses expériences (Gillies et Robinson, 2012). D’autres, au contraire, critiquent la mise en place 
de méthodologies adaptées et soutiennent que les enfants n'ont pas une vision du monde fondamentalement différente de celle des adultes qui les entourent (Samuelsson et coll., 2015; Sarcinelli, 2015). Par ailleurs, pour Sarcinelli (2015 : 15), il est alors contre-productif d'opposer le consentement des adultes à l'assentiment des enfants et il n'appartient pas au chercheur de «s'arroger le droit de tracer la ligne de démarcation entre les droits de l'enfant et celui de ses parents».

Un positionnement mitoyen a constitué notre ligne de conduite dans le cadre du présent projet de recherche. Alors que les parents ont été consultés et informés des tenants et aboutissants de la participation de leurs enfants et leur ont permis de rencontrer les chercheures, ce sont les enfants qui ont eu le dernier mot quant à leur implication. Cette démarche particulière, portée sur l'assentiment des enfants comme conditions sine qua non à la participation, nous démontre que les enfants sont capables d'agentivité et peuvent se positionner et exprimer leur désir de participer ou non à un projet de recherche.

En outre, notre expérience démontre qu'une méthodologie adaptée permet à un enfant de comprendre la nature de sa participation à un projet de recherche et d'y assentir par lui-même, nonobstant ce qu'en pensent ses parents. Les outils utilisés et la procédure déployée rééquilibrent le pouvoir entre le chercheur et l'enfant tout en lui conférant un sentiment d'autogouvernance. Le fait que certains participants aient exprimé explicitement ou implicitement leur dissentiment - parfois même en cours de processus - nous permet de croire que l'information qui leur est transmise quant aux caractéristiques de l'assentiment (droit de retrait en tout temps, réassurance quant à la possibilité de déplaire, etc.) est appropriée. Par ailleurs, le fait d’omettre toute référence à la récompense avant la fin du processus exclut que cela agisse en tant que facteur extrinsèque faussant l'assentiment. Enfin, le fait d'avoir à signer la lettre de permission a été perçu favorablement par les enfants rencontrés. Le mot «permission», à connotation clairement enfantine, a également très bien été intégré.

Il reste néanmoins que nous nous questionnons sur la transférabilité de ces outils à l'ensemble des enfants participant à un projet de recherche, et ce, du fait de particularités liées à notre cohorte. D’une part, nos jeunes participants sont issus de familles déjà impliquées dans les projets de recherche de deux chercheures. Ce faisant, les parents sont socialisés quant au processus de recherche, connaissent bien les chercheures concernées puisqu'ils ont déjà agi eux-mêmes à titre de participants et adhèrent aux objectifs de ces études. Ils ont donc pu avoir une influence positive sur la motivation de leurs enfants à assentir à participer, afin de pouvoir, eux aussi, «faire comme papa(s) ou maman(s)». Il est difficile, dans ce contexte, d'évaluer si leur participation est libre de ce biais. D’autre part, ces enfants proviennent majoritairement de milieux socialement et culturellement favorisés, ce qui fait que les outils utilisés, basés sur la lecture, leur étaient facilement accessibles. Si Ford, Sankey et Crisp (2007) soutiennent que les enfants n'ont pas besoin d'habiletés spécifiques en lecture, mais plutôt d'une capacité à comprendre l'information qui leur est transmise de la part du chercheur, il n'en reste pas moins que ce type d'outil doit faire l'objet de recherche auprès de différents groupes d'enfants pour en confirmer la validité. À ce propos, une étude en cours (Gervais, Côté, De Montigny et coll., 2016-18) portant sur les représentations d'enfants immigrants de l'engagement paternel permettra d'attester ou non la pertinence de nos choix méthodologiques, puisqu'elle s'appuie sur les mêmes procédures et outils d'assentiment que la présente recherche. 


\section{Conclusion}

L'assentiment auprès des enfants recèle des enjeux importants. L'âge de compréhension de l'enfant, la relation de pouvoir entre le chercheur et le participant, ainsi que les incitatifs à la participation sont autant d'éléments qui exigent une réflexion approfondie et continue de la part des membres de l'équipe de recherche. La mise en place de conditions favorables pour promouvoir l'assentiment libre et éclairé des enfants doit demeurer une préoccupation constante, et ce, tout au long du processus. Ce souci éthique nous semble primordial afin d'instaurer un dialogue empreint de respect avec les enfants.

Basés sur les principes théoriques et méthodologiques de l'approche centrée sur l'enfant, nos outils d'assentiment ont été développés dans le but d'améliorer la compréhension des enfants et de favoriser leur implication. L'analyse rétrospective de notre stratégie de recherche nous indique que l'utilisation d'outils flexibles, telles la lettre d'information et l'activité de permission, semble avoir eu un impact positif sur le déroulement de l'étude. Les ajustements déployés à chaque étape, de la conception des outils basée sur les savoirs existants à leur expérimentation lors de la collecte des données, montrent le caractère itératif et l'espace créatif que nous avons aménagé tout au long du processus de recherche. 


\section{Bibliographie}

Alderson, P., et Morrow, V. (2011). The ethics of research with children and young people. A practical handbook. Los Angeles, London, New Delhi, Singapore, Washington DC : Sage.

Aubrey, C., et Dahl, S. (2006). Children's voices: The views of vulnerable children on their service providers and the relevance of services they receive, British Journal of Social Work, 36(1), 21-39.

Bray, L. (2007). Developing an activity to aid informed assent when interviewing children and young people. Journal of Research in Nursing, 12(5), 447-457.

Burke, T.M., Abramovitch, R., et Zlotkin, S. (2005). Children's understanding of the risks and benefits associated with research, Journal of Medical Ethics, 31(12), 715-720.

Bushin, N. (2007). Interviewing with children in their homes: Putting ethical principles into practice and developing flexible techniques. Children's geographies, 5(3), 235-251

Carroll-Lind, J., Chapman, J.W., Gregory, J., et Maxwell, G. (2006). The key to the gatekeepers: Passive consent to and other ethical issues surrounding the rights of children to speak on issues that concern them. Child Abuse \& Neglect, 30(9), 379-389

Cashmore, J. (2011). Children's participation in family law decision-making: Theoretical approaches to understanding children's views. Children and Youth Services Review, 33(4), 515-520.

Christensen P., M., et Prout, A. (2010). Anthropological and sociological perspectives on the study of children, dans S. Grenne et D. Hogan (Éds). Researching children's experience (p. 42-60). Los Angeles, London, New Deli, Singapore, Washington DC : Sage.

Comité d'éthique de la recherche en santé (CÉRES), Comité plurifacultaire d'éthique de la recherche (CPÉR) Comité d'éthique de la recherche en arts et en sciences (CÉRAS) (2014). Guide d'information sur le consentement libre, éclairé et continu. Repéré à http://www.recherche.umontreal.ca/fileadmin/user_upload/ Ethique_humaine/CPER/Guide_FCLE.pdf

Delalande, Danic, I. et Rayou, P. (2006). Enquêter auprès d'enfants et de jeunes: objets, méthodes et terrains de recherche en sciences sociales. Rennes: Presses Universitaires de Rennes.

Diekema, D.S (2003). Taking Children Seriously: What's so important about Assent? The American Journal of Bioethics 3(4), 25-26.

Ducan, R.E., Drew, S.E., Hogdson, J., et Sawyer, S. M. (2009). Is my mum going to hear this? Methodological and ethical challenges in qualitative health research with young people, Social Science and Medecine, 69(11), 1691-1699.

Emond, R. (2006). Reflections of a researcher on the use of a child-centred approach. The Irish journal of psychology, 27(1-2), 97-104.

Fargas-Malet, M., McSherry, D., Larkin, E. et Robinson, C. (2010). Research with children: methodological issues and innovative techniques. Journal of Early Childhood Research, 8(2), 175-192.

Fernandez, C. (2003). Context in shaping the ability of a child to assent to research. American journal of bioethics, 3(4), 29-30.

Ford, K., Sankey, J. et Crisp, J. (2007). Development of children's assent documents using a child-centred approach. Journal of child health care, 11(1), 19-28. 
Fundudis,T. (2003) Consent issues in medico-legal procedures: how competent are children to make their own decisions? Child Adolescent Mental Health, 8(1), 18-22

Gallagher, M. (2009). Ethics. Dans E. Kay, M. Tisdall, J.M. Davis et M. Gallagher (Eds). Researching with children \& young people. Research design, Methods and Analysis (p. 11-28). Los Angeles-London-New Deli-Singapore-Washington DC, Sage.

Gallagher, M., Haywood, S., Jones, M., et Milne, S. (2010). Negotiating informed consent with children in school-based research: A critical review. Children and Society, 24(6), 471-482.

Gervais, C., Côté, I., De Montigny, F., Ndengeyingoma, A. et Tardif-Grenier, K. (2016-2018). Relations familiales et engagement paternel en contexte migratoire: Représentations. Subvention obtenue dans le cadre du programme Développement-Savoir du Conseil de Recherches en Sciences Humaines du Canada (CRSH)

Gillies, V. et Robinson, Y. (2012). Developing creative research methods with challenging pupils. International Journal of Social Research Methodology, 15(2), 161-173.

Graham, A., Powell, M.A., et Taylor, N. (2015). Ethical research involving children : Encouraging reflexive engagement with children and young people. Children \& Society, 29(5), 331-343.

Graham, A., Powell, M.A, Taylor, N., Anderson, D. et Fitzgerald, R. (2013). Recherche éthique impliquant des enfants. Florence, Centre de recherche de l'UNICEF - Innocenti.

Harcourt, D. et Einarsdottir, J. (2011). Introducing children's perspectives and participation in research. European Early Childhood Education Research Journal, 19(3), 301-307.

Hill, M., Davis, J., Prout, A., et Tisdall, K. (2004). Moving the participation agenda forward. Children and Society, 18(2), 77-96.

Hodson, L. (2012). Ties That Bind: Towards a Child-Centred Approach to Lesbian, Gay, Bi-Sexual and Transgender Families under the ECHR*. International Journal of Children's Rights, 20(4), 501-522.

Kirk, S. (2007). Methodological and ethical issues in conducting qualitative research with children and young people: A literature review. International Journal of Nursing Studies, 44(7), 1250-1260.

Lambert, V. et Glacken, M. (2011). Engaging with children in research: Theoretical and practical implications of negotiating informed consent/assent. Nursing Ethics, 18(6), 781-801.

Lépine, R., Saint-Jacques, M.-C., Champoux, L., et Lessard, G. (2011). Une collecte de donnée efficace et sécuritaire auprès des jeunes et des familles. Collection «Devenir chercheur», Québec, QC : Centre de recherche sur l'adaptation des jeunes et des familles à risque de l’Université Laval.

Lewis, A. et Porter, J. (2004). Interviewing children and young people with learning disabilities*: guidelines for researchers and multi-professional practice. British Journal of Learning Disabilities, 32(4), 191-197.

Lundy, L. et McEvoy, L. (2011). Children's rights and research processes: Assisting children to (in)formed views. Childhood, 19(1), 129-144.

Mason, J., et Hood, S. (2011). Exploring issues of children as actors in social research. Children and youth services review, 33(4), 490-495.

Meaux, J.B. et Bell, P.L. (2001). Balancing recruitment and protection: Children as research subjects. Comprehensive Pediatric Nursing, 24(4), 241-251.

Miller, V., Drotar, D. et Kodish, E. (2004). Children's Competence for Assent and Consent: A Review of Empirical Findings. Ethics and Behavior, 14(3), 255-295. 
Miller,V.et Nelson, R. (2006) A developmental approach to child assent for non-therapeutic research. The Journal of Pediatrics, 149(1), S25-S30.

Montero, M. (2009). Community action and research as citizenship construction. American Journal of Community Psychology, 43(1-2), 149-161.

Parizeau, M.-H. (2015). L'enfant et la recherche biomédicale : entre autonomie progressive et devoir de protection de vulnérabilité, dans C. Landheer-Cieslak et L. Langevin (Éds). La personne humaine entre autonomie et vulnérabilité. Mélanges en l'honneur d'Édith Deleury (p. 513-540), Montréal : Yvon Blais.

Powell, M.A., Fitzgerald, R., Taylor, N.J., et Graham, A. (2012). International Literature Review: Ethical Issues in Undertaking Research with Children and Young People (Literature review for the Childwatch International Research Network). Lismore: Southern Cross University, Centre for Children and Young People / Dunedin: University of Otago, Centre for Research on Children and Families.

Razy, É. (2014). Éthique et enfants. Des habits neufs pour de vieilles questions anthropologiques? AnthropoChildren, 4, Repéré à http://popups.ulg.ac.be/2034-8517/index.php?id=1991

Renou, M. (2005). Psychoéducation: une conception, une méthode. Éditions Sciences et Culture: Montréal.

Rice, M., et Broome, M. (2004). Incentives for children in research. Journal of Nursing Scholarship, 36(2), 167-172.

Rossi, W.C., Reynolds, W. et Nelson, R.M. (2003) Child assent and parental permission in pediatric research. Theoretical Medicine, 24(2), 131-148.

Samuelsson, T., Sparrman, A., Cardell, D., et Lindgren, A.-L. (2015). The active, competent child, capable of autonomous action: An inherent quality or the outcome of a research process? AnthropoChildren, 5, Repéré à http://popups.ulg.ac.be/2034-8517/index.php?id=2370

Sarcinelli, A.S. (2015). Réflexions épistémologiques sur l'ethnographie de l'enfance au prisme des rapports d’âge, AnthropoChildren, 5, Repéré à http://popups.ulg.ac.be/2034-8517/index.php?id=2241

Starkey, H., Akar, B., Jerome, L. et Osler, A. (2014). Power, pedagocy and participation: ethics and pragmatics in research with young people. Research in Comparative and International Education, 9 (4), 426-440.

Spyrou, S. (2011). The limits of children's voices: From authenticity to critical, reflexive representation. Childhood, 18(2) 151-165.

Tait, A.Voepel-Lewis,T., Malviya, S. (2003) Do they understand? (Part II): Assent of children participating in clinical anaesthesia and surgery research. Anesthesiology, 98, 609-614.

Tisdall, E.K.M. et Punch, S. (2012). Not so 'new'? Looking critically at childhood studies, Children's Geographies, 10(3), 249-264.

Twycross, A., Gibson, F. et Coad, J. (2008). Guidance on seeking agreement to participate in research from young children. Pediatric nursing, 20(6), 14-18.

Willumsen, E., Vegar Hugaas, J. et Studsrør, I. (2014). The Child as Co-researcher. Moral and Epistemological Issues in Childhood Research. Ethics and Social Welfare, 8(4), 332-349. 
TITRE: RELATION DE CONFIANCE, UN ANCRAGE INCONTOURNABLE EN CONTEXTE DE RECHERCHE AUTOCHTONE : TÉMOIGNAGES DE RECRUTEMENT DE PARTICIPANTS ATIKAMEKWS, INUIT, ANICINAPEK ET INNUS EN ÉDUCATION

Auteur(s): Yvonne da Silveira, Elisabeth Jacob, Glorya Pellerin et Véronique Paul

PublicATION: RECRUTEMENT ET CONSENTEMENT À LA RECHERCHE : RÉALITÉS ET DÉFIS ÉTHIQUES

PAGES: $146-160$

ISBN: 978-2-7622-0357-8

Directeurs: Ana Marin, BÉatrice Eysermann et Michel T. GirouX

URI: HTTP://HDL.HANDLE.NET/11143/14113

DOI: HTTPS://DOI.ORG/10.17118/11143/14113 


\section{Relation de confiance, un ancrage incontournable en contexte de recherche autochtone : témoignages de recrutement de participants atikamekws, inuit, anicinapek et innus en éducation ${ }^{1}$}

Yvonne da Silveira, Ph. D., Professeure retraitée, Unité d'enseignement et de recherche en sciences de l'éducation, Université du Québec en Abitibi-Témiscamingue

Elisabeth Jacob, Ph. D., Professeure en éducation préscolaire, Département des sciences de l'éducation, Université du Québec à Chicoutimi

Glorya Pellerin, Ph. D., Professeure, Unité d'enseignement et de recherche en sciences de l'éducation, Université du Québec en Abitibi-Témiscamingue

Véronique Paul, agente de recherche, Unité de recherche, de formation et de développement en éducation en milieu inuit et amérindien, Université du Québec en Abitibi-Témiscamingue

Résumé : Basé sur trois recherches liées à la pratique enseignante, cet article est constitué de témoignages sur le processus de recrutement de participants en contexte autochtone. Il souligne l'importance d'une démarche de recherche collaborative ou de recherche-action tenant compte des besoins des milieux scolaires concernés, la nécessité d'une relation préexistante avec les enseignantes participantes ou au moins d'une rencontre en personne pour l'établissement de la recherche. Un point commun et incontournable à ces recherches demeure la relation de confiance. Ces éléments font écho à des recommandations d'approche de recherche émanant du paradigme de recherche autochtone. La conclusion suggère entre autres que le recrutement de participants et la collaboration, sous-tendus par le consentement, vont de pair avec la relation de confiance qui doit, dès le départ, être établie entre tous les membres de l'équipe de recherche.

Mots-clés : relation de confiance, contexte de recherche autochtone, pratique enseignante

Abstract : Drawing on three studies of teaching practice, we report on the process of recruiting participants in an Indigenous setting. This paper stresses the importance of a collaborative or action-research approach that takes into account the needs of the school environments under study. It also stresses the necessity of a pre-existing relationship with participating teachers or at least a face-to-face meeting before research begins. These studies agree on one key thing: a relationship of trust. The above points echo recommendations on research approaches that come from the Indigenous research paradigm. In conclusion, we notably suggest that participant recruitment and collaboration, based on consent, go hand in hand with a relationship of trust, which should be established from the outset between everyone on the research team.

Key words: relationship of trust, Indigenous research context, teaching practice

1. Respectant les choix de chacune des communautés concernées, les trois règles d'accords suivantes ont été privilégiées : 1) atikamekw au singulier, atikamekws au pluriel; 2) invariabilité du mot inuit, étant donné que ce terme désigne déjà plusieurs personnes en inuktitut (cf. Dorais, L.-J., 2004) anicinape au singulier, anicinapek au pluriel. Pour ce qui est du mot innu, nous respectons les règles d'accords de l'Office Québécois de langue française concernant le féminin et le pluriel. 


\section{Introduction}

Mener une recherche dans une communauté autochtone exige que les membres de la nation se sentent en confiance et que le projet reçoive l'approbation du conseil de bande. Nos expériences de recherche en éducation en contexte autochtone nous amènent à approuver l'affirmation voulant que «le recrutement va de pair avec le consentement» ${ }^{2}$ et ainsi, la position de la Food and Drug Administration (FDA) américaine liant ces deux dimensions.

Notre article est constitué de trois témoignages décrivant trois expériences vécues du processus de recrutement de participants en contexte de recherche autochtone. Le premier provient d'une recherche doctorale (en cours au moment de la communication et soutenue en septembre 2017) portant sur les rôles des enseignantes autochtones à l'éducation préscolaire pour favoriser l'émergence de l'écrit en contexte de jeu symbolique (Jacob, E.); le deuxième découle d'une recherche visant la mise en place d'une approche d'accompagnement à la formation des enseignants inuit par la visioconférence (Pellerin, G.); et le troisième, d'une recherche qui a porté sur l'exploration de nouvelles pratiques d'enseignement pour favoriser le développement de la compétence à écrire d'élèves autochtones du primaire (da Silveira, Y.) $)^{3}$.

Ces recherches sont menées en éducation et sont en lien avec la pratique enseignante. La démarche méthodologique adoptée en est une de recherche collaborative et de recherche-action. Les moments clés de ces expériences reflétant une relation de confiance, notamment lors du recrutement des participants de la recherche, nous les avons soulignés. Ils sont par la suite reliés à des éléments du paradigme de recherche autochtone afin de dégager leurs affinités avec ceux de la recherche scientifique. Notre conclusion propose des pistes de réflexion concernant la spécificité de la recherche en contexte autochtone et certains aspects du recrutement de participants.

2. Eysermann, B. et Marin, A. (2016). Citation extraite du résumé de l'annonce du colloque joint au courriel d'invitation reçu le 11 février 2016 : Colloque/ACFAS 2016. Appel à contributions. "Seriez-vous intéressé par mon projet de recherche? » Sortir des sentiers battus : les défis du recrutement à la recherche.

3. (1) Les rôles des enseignantes atikamekws à l'éducation préscolaire pour favoriser l'émergence de l'écrit en contexte de jeu symbolique. Thèse de doctorat d'E. Jacob. Université du Québec à Montréal. Subventionnée par le Fonds de recherche du Québec - Société et culture (FRQSC) et le Conseil de recherches en sciences humaines du Canada (CRSH) 2012 à aujourd'hui. (2) Projet de mise en place d'une approche d'accompagnement à la formation des enseignants Inuit par la vidéoconférence. Chercheuse Principale : Glorya Pellerin (UQAT). Cochercheurs: Yvonne da Silveira et Gisèle Maheux (UQAT), Stéphane Allaire (UQAC), Siaja Mark (École Nuvviti), Elisapi Uitangak (École Ikaarvik). Projet de recherche subventionné par le Conseil de recherches en sciences humaines du Canada (CRSH) 2011-2016. (3) Exploration de nouvelles pratiques d'enseignement pour favoriser le développement de la compétence à écrire d'élèves anicinapek et innus du primaire. Chercheuse Principale : Yvonne da Silveira (UQAT). Cochercheuses : Glorya Pellerin, Gisèle Maheux (UQAT), Mirela Moldoveanu (UQAM), Christiane Blaser (U. Sherbrooke) Projet de recherche subventionné par le Fonds de recherche du Québec - Société et culture (FRQSC) 2012-2015. 


\section{Contextes respectifs des trois recherches}

\section{Les rôles des enseignantes autochtones à l'éducation préscolaire pour favoriser l'émergence de l'écrit en contexte de jeu symbolique}

À l'éducation préscolaire, les enseignants autochtones jouent un rôle important dans la découverte et le développement de l'écrit des enfants. De l'avis de nombreux leaders du monde de l'éducation, notamment en contexte autochtone, il est souhaitable que l'entrée dans l'écrit des enfants se fasse dans leur langue maternelle afin de revitaliser les langues autochtones et de faciliter le passage de l'apprentissage en langue seconde (Ball, 2009; Francis et Revhner, 2002). Ces enseignants font donc face à des défis supplémentaires puisqu'il existe encore très peu de livres écrits en langue autochtone destinés aux jeunes enfants. Un des moyens est alors d'utiliser le jeu symbolique pour permettre aux enfants d'adopter des comportements de lecteur et de scripteur dans un contexte ludique et significatif pour eux. Or, les enseignants autochtones à l'éducation préscolaire, voire les enseignants en général, peuvent se sentir peu outillés pour combiner l'approche du jeu symbolique et celle de l'émergence de l'écrit, notamment parce que ces thèmes sont peu abordés dans la formation initiale à l'enseignement.

L'objectif général de la recherche doctorale de Jacob (2017 : 88) est «d'analyser les rôles des enseignantes atikamekws à l'éducation préscolaire pour favoriser l'émergence de l'écrit en contexte de jeu symbolique». Cette recherche collaborative a été menée avec trois enseignantes atikamekws à l'éducation préscolaire dans une école primaire d'une communauté atikamekw. Dans ce projet, une activité réflexive enseignante/chercheuse a été mise en place. Cette activité consistait à planifier des coins de jeu symbolique enrichis de matériel écrit, à réfléchir à ce qu'il est possible de faire pour favoriser la lecture et l'écriture dans ces coins, à faire faire une mise en pratique dans la classe aux enseignantes en interaction avec les enfants et à réaliser un retour réflexif (chercheuse/enseignantes) pour permettre à chacune des enseignantes d'expliciter sa pratique. Durant la mise en pratique, la chercheuse était présente pour noter des observations et accompagner les enseignantes, selon leurs besoins, lorsqu'elles jouaient avec les enfants de la classe. Ainsi, quatre coins de jeu enrichis de matériel écrit ont été planifiés et mis en pratique dans la classe sur les thèmes suivants : le restaurant, l'hôpital, la construction et la chasse. Par exemple, pour le coin de l'hôpital, des crayons, des revues, des carnets de prescription, des affiches du corps humain avec des mots écrits en atikamekw ont été mis à la disposition des enfants. Pour chacun des coins, la langue orale et écrite privilégiée était l'atikamekw, puisqu'il s'agit de la langue d'enseignement du préscolaire à la deuxième année du primaire dans l'école concernée dans la recherche.

\section{Profil de recrutement}

Pour mener à terme ce projet doctoral, Jacob a recruté les participantes en différentes étapes couvrant une période d'environ deux ans. Avant de commencer sa recherche, la chercheuse travaillait déjà comme consultante en petite enfance pour la communauté atikamekw concernée. Un lien de confiance était déjà créé avec les membres de la communauté, les intervenants scolaires et les enseignantes. Durant les années antérieures au projet de recherche, les enseignantes atikamekws à l'éducation préscolaire avaient exprimé à la consultante des besoins de formation concernant le programme d'éducation préscolaire du Programme de formation de l'école québécoise (Ministère de l'Éducation du Québec, 2001 : 60), notamment en ce qui a trait à la compétence 4, «communiquer en utilisant les ressources de la langue». Ces enseignantes désiraient valoriser le langage oral et écrit atikamekw dans leur classe. C'est donc à partir des besoins exprimés par les enseignantes qu'un projet de recherche est né, réunissant les thèmes du jeu symbolique et de l'émergence de l'écrit. De plus, 
ces thèmes rejoignaient la préoccupation de la consultante et chercheuse pour ses études doctorales. Cette dernière a donc pu mieux concevoir l'objet de sa recherche, tout en étant à l'écoute des besoins des enseignantes. D’ailleurs, les enseignantes ont aussi exprimé un besoin de matériel de jeu et de matériel écrit pour leur classe. Pour cela, la recherche collaborative a été choisie comme méthodologie puisque celle-ci répondait au besoin de formation exprimé par les enseignantes et à celui d'enrichir les classes de matériel de jeu.

Même si le recrutement des participantes s'est réalisé sur la base d’une relation préexistante et répondait aux besoins des enseignantes, la chercheuse devait respecter les principes déontologiques de la recherche et obtenir le consentement du conseil de bande de la communauté afin d'effectuer une recherche sur son territoire. Ce consentement répond aux exigences de l'Énoncé de politique des trois Conseils : Éthique de la recherche avec des êtres humains (EPTC2, 2014) concernant toute recherche en milieu autochtone. Pour cela, la chercheuse a obtenu l'appui du coordonnateur de l'éducation spécialisée, du directeur de l'école primaire ainsi que du directeur à l'éducation de la communauté concernée. Le projet de recherche a ensuite été présenté aux membres du conseil de bande qui ont entériné la demande de la chercheuse et autorisé la réalisation de la recherche. Enfin, la dernière préoccupation de la chercheuse pour le recrutement des participantes était d'obtenir leur consentement de façon libre et éclairée. Avant la réalisation du projet de recherche, une rencontre a eu lieu dans la communauté avec les enseignantes afin de leur expliquer le projet, de le modifier le cas échéant afin qu'il réponde une fois de plus à leurs besoins pour ensuite obtenir leur consentement. Cette rencontre a également permis de déterminer l'horaire des activités de recherche avec les enseignantes, de préciser leurs attentes et d'exprimer leurs craintes. À la fin de cette rencontre, les trois enseignantes à l'éducation préscolaire ont accepté librement de signer le formulaire de consentement.

\section{Projet de mise en place d'une approche d'accompagnement à la formation des enseignants inuit par la vidéoconférence}

Le Nunavik compte 14 villages inuit dispersés le long des côtes de la Baie d'Hudson et de la Baie d'Ungava. Tous isolés les uns des autres, ces villages ne sont accessibles que par bateau ou par avion. Chaque communauté dispose au moins d'une école où l'enseignement est dispensé uniquement dans la langue maternelle, l'inuktitut, du préscolaire à la $2^{e}$ année du primaire. À partir de la $3^{e}$ année, les enfants sont scolarisés partiellement ou totalement en langue seconde, français ou anglais, mis à part des cours associés à la langue et à la culture inuit. Les Inuit du Nunavik ont commencé à exercer la fonction d'enseignant dans les écoles de leur communauté au début des années 1970, époque où on leur confia l'enseignement de leur langue et de leur culture, ce qu'ils assurent encore aujourd'hui (Cram, 1985).

En 1984, une collaboration entre les communautés d'Ivujivik, de Puvirnituq et l'Université du Québec en Abitibi-Témiscamingue (UQAT) s'instaura à la demande des leaders inuit en éducation de ces communautés; un programme de formation des enseignants respectant un principe de cogestion en partenariat ${ }^{4}$ en émergea. Ce programme a pour objectif d'offrir aux enseignants inuit une formation professionnelle qui répond aux normes de la province de Québec, tout en tenant compte des besoins particuliers issus du contexte géographique et culturel singulier dans lequel il s'insère. De ce fait, le programme prend en considération que les enseignants inuit en poste au Nunavik n'ont pas fréquenté les institutions de formation initiale à l'enseignement avant leur entrée en fonction à l'école, mais sont des membres de la communauté dont les compétences sont reconnues par les responsables de l'éducation du milieu. En effet, la plupart des enseignants inuit en exercice s'initient

4. La gestion du programme est réalisée en partenariat par une équipe formée des professeurs de l'UQAT et des partenaires des deux communautés. Le programme de formation, incluant les activités de formation elles-mêmes, leur développement et leur gestion, constitue l'objet principal des interactions entre les partenaires engagés dans le processus. 
d'abord à la pratique en assistant un enseignant titulaire et acquièrent leur formation professionnelle initiale, menant à un certificat d'enseignement, à temps partiel directement dans les communautés.

Depuis 1984, les activités de formation sont offertes dans une des communautés à raison de deux séjours d'une semaine au cours de laquelle 22-23 heures de cours sont dispensées par 1) un professeur ou un chargé de cours de l'UQAT qui se déplace au Nord et 2) un coenseignant inuit qui assiste le professeur dans la préparation et le pilotage des cours, en traduisant et en explicitant les contenus en inuktitut aux étudiants qui, rappelons-le, sont des enseignants en formation.

C'est dans la poursuite de ce projet de formation des enseignants inuit que s'insère le projet ${ }^{5}$ de mise en place d'une approche d'accompagnement à la formation des enseignants inuit par la vidéoconférence, une recherche collaborative à laquelle se sont associés les partenaires institutionnels suivants : la Commission scolaire Kativik, Tamaani6 et l’Université du Québec en Abitibi-Témiscamingue.

Afin de mieux comprendre le contexte de la recherche, notons qu'au cours de la dernière décennie les moyens de communication ont évolué rapidement et que cette région de la province n'y a pas échappé. II est alors apparu évident pour les membres du groupe de cogestion, tant inuit qu'universitaires, qu'utiliser des moyens technologiques permettrait de mieux répondre aux besoins d'accompagnement des étudiants en formation. Ainsi, la visioconférence a été identifiée comme un moyen de communication pertinent à développer au Nord afin d'améliorer les contacts entre les professeurs, les coenseignants inuit et les étudiants. Le projet a donc pris forme en 2010, grâce à une étroite collaboration entre l'université, les communautés partenaires, et des organisations inuit régionales éducatives et administratives. Le projet poursuivait entre autres comme objectifs d'identifier les conditions optimales de formation des enseignants inuit par une expérience pratique d'utilisation de la visioconférence en complément à la formation offerte en présentiel, de développer une expertise de recherche inuit ancrée dans les communautés et d'approfondir les connaissances et les pratiques professionnelles des enseignants par leur mise en réseau. Le projet de recherche a ainsi mené à la mise en place d'un système technologique qui favorise dorénavant une communication fluide entre les étudiants et les formateurs, et ce, malgré la distance.

\section{Profil de recrutement}

Les participants à la recherche étaient des étudiants et des formateurs des programmes de formation des enseignants inuit de l'UQAT. Comme cette université compte plus de trente ans de partenariat avec les communautés de Puvirnituq et d'Ivujivik, une relation de confiance était déjà établie entre les participants, tant inuit qu'universitaires. Ces derniers partageaient un besoin commun d'expérimenter de nouvelles formules d'enseignement pouvant contribuer à enrichir la formation des enseignants inuit, notamment par la variation des formules pédagogiques et l'amélioration de l'accompagnement offert aux étudiants, permettant ainsi des rencontres plus fréquentes malgré la distance.

Afin d'assurer le recrutement des collaborateurs du projet, différentes actions ont été menées. D’abord, une tournée des écoles concernées a été réalisée, au cours de laquelle la chercheuse principale a pu prendre le temps d'échanger sur les objectifs poursuivis, de présenter le déroulement anticipé, de rassurer les milieux scolaires, de valider les possibilités d'utilisation de la technologie et de demander l'approbation du projet auprès

5. Projet décrit à la troisième note.

6. Tamaani est une des sections de l'Administration régionale Kativik (ARK) et agit à titre de fournisseur des services internet sans fil au Nunavik. 
de chacun des partenaires. L'équipe de recherche a dès lors été formée en respectant deux grands principes: une participation qui, premièrement, illustre la représentativité de chaque milieu et, deuxièmement, est basée sur l'intérêt à la recherche et sur le volontariat de ses membres.

Suite au recrutement des collaborateurs du projet, des actions ont été posées pour assurer le recrutement des participants. Cette opération a été réalisée par les collaborateurs des milieux qui ont contacté les étudiants afin de solliciter leur participation. L'ensemble des participants inscrits dans les programmes de formation des enseignants inuit ont accepté de participer au projet. La collaboration a été entretenue par des rencontres mensuelles au cours desquelles les commentaires des collaborateurs ont été pris en compte pour réajuster l'approche au fil du développement du projet. Des rencontres annuelles ont également été organisées avec les partenaires institutionnels du projet afin de les informer du déroulement, mais aussi pour effectuer certains réajustements, le cas échéant.

\section{L'exploration de nouvelles pratiques d'enseignement pour favoriser le développement de la compétence à écrire d'élèves anicinapek et innus du primaire}

Les pratiques culturelles autochtones ont été historiquement ancrées dans l'oralité alors que l'éducation scolaire occidentale impose l'écrit. Cette réalité accentue le phénomène de développement bilingue inhérent au contact de la langue maternelle autochtone et de la langue seconde, en l'occurrence le français, ainsi que l'écart entre culture orale et culture écrite en contexte autochtone. Elle engendre un rapport singulier de l'élève à la langue d'enseignement et peut provoquer d'importantes difficultés d'apprentissage scolaires. L'écriture étant un pilier de la réussite et de la persévérance scolaires, l'enseignement de l'écrit pose ainsi des défis particuliers dans ce contexte. Cette recherche a donc eu pour objectif général de contribuer au développement de la compétence scripturale d'élèves autochtones en français langue d'enseignement par l'expérimentation de pratiques d'écriture adaptées au contexte en intervenant auprès des enseignantes qui travaillent au quotidien avec ces élèves, et cela, en partenariat avec des collaboratrices des milieux scolaires.

Menée dans les deux communautés autochtones - anicinape de Pikogan et innue d'Unamen Shipu (La Romaine) - elle s'est concrétisée à travers les objectifs spécifiques suivants : identifier le rapport à l'écrit d'élèves autochtones de ces communautés; identifier les principales difficultés que ces élèves éprouvent dans leur pratique d'écriture; accompagner des enseignantes dans l'élaboration et l'expérimentation de pratiques d'écriture qui tiennent compte des caractéristiques de la population scolaire ciblée; et évaluer, au moyen du portfolio, les effets des pratiques expérimentées sur le développement de la compétence scripturale des élèves autochtones et sur leur rapport à l'écrit. L'atteinte de ces objectifs exigeait un travail étroit de collaboration entre chercheurs et enseignants dont a été consciente l'équipe de recherche qui a choisi la recherche-action comme mesure nécessaire pour y arriver (Da Silveira et coll., 2015).

\section{Profil de recrutement}

Aussitôt l'annonce du thème de recherche de l'organisme subventionnaire FRQSC, soit Actions concertées Programme de recherche sur l'écriture, la chercheuse principale a partagé l'idée du projet de recherche avec des cochercheuses potentielles. Elle a ensuite eu une communication téléphonique avec deux anciennes collaboratrices de l'école de Pikogan avec lesquelles elle avait travaillé à une recherche en partenariat ${ }^{7}$ quelques années plus tôt afin de vérifier leur intérêt à prendre part à une telle recherche. Le désir d'y participer était

7. Maheux, da Silveira et Dorais (2005) «Interface autochtones-université en formation des enseignants : mise en place de partenariats de recherche avec des organismes cris, algonquins et inuit ». Programme de subvention CRSH - Réalités autochtones. 
manifeste chez ces deux personnes qui lui avaient alors confié qu'une telle problématique rejoignait l'une de leurs préoccupations en contexte autochtone. De plus, les deux anciennes collaboratrices lui ont souligné leur confiance pour la suite des choses, arguant qu'elles la connaissaient déjà et qu'elles n'en étaient pas à leur première collaboration. Notons qu'avant leur première collaboration en recherche ces deux dames anicinapek, alors étudiantes du programme de maîtrise de l'UQAT, et la chercheuse principale avaient eu l'opportunité de se rencontrer à travers des cours. Cet intérêt des anciennes collaboratrices anicinapek s'est matérialisé par des échanges et la prise en compte de leurs commentaires tout comme de ceux des cochercheuses universitaires dans le contenu de la lettre d'intention de recherche envoyée au FRQSC.

Ainsi, à la base de cette recherche, il y a eu une équipe de cinq chercheuses universitaires provenant de trois universités (UQAT, UQAM et Université de Sherbrooke) à laquelle se sont jointes trois collaboratrices, incluant les deux Anicinapek, de trois écoles primaires qui ont joué un rôle d'interface entre l'équipe de chercheuses et six enseignantes participantes.

À la différence des deux premiers témoignages rapportés ci-dessus pour le recrutement des participantes, il y a eu dans cette recherche-ci trois profils de recrutement qui reflètent trois relations différentes entre les chercheuses et les terrains de recherche concernés. Le tableau suivant en donne un aperçu.

\section{Tableau 1 - Trois profils de recrutement - Trois relations différentes}

\begin{tabular}{|c|c|c|c|}
\hline $\begin{array}{l}\text { Étapes de déroulement } \\
\text { de la recherche/ } \\
\text { Implication des collaboratrices et } \\
\text { des enseignantes participantes }\end{array}$ & $\begin{array}{l}\text { Contexte anicinape } \\
\text { (Pikogan) }\end{array}$ & $\begin{array}{l}\text { Contexte innu } \\
\text { (La Romaine) }\end{array}$ & $\begin{array}{l}\text { Contexte cri } \\
\text { (Chisasibi) }\end{array}$ \\
\hline Lettre d'intention & $\begin{array}{l}\text { Collaboratrices du milieu } \\
\text { scolaire }\end{array}$ & Aucune participation & Aucune participation \\
\hline Demande de subvention & $\begin{array}{l}\text { Collaboratrices du milieu } \\
\text { scolaire }\end{array}$ & $\begin{array}{l}\text { Collaboratrice du milieu } \\
\text { scolaire }\end{array}$ & $\begin{array}{l}\text { Collaborateur du } \\
\text { milieu scolaire }\end{array}$ \\
\hline Certificat d'éthique; Résolution conseils de bande & $\begin{array}{l}\text { Collaboratrices du milieu } \\
\text { scolaire }\end{array}$ & $\begin{array}{l}\text { Collaboratrice du milieu } \\
\text { scolaire }\end{array}$ & Ne s’applique pas \\
\hline Recrutement & $\begin{array}{l}\text { Collaboratrices du milieu } \\
\text { scolaire et chercheuse }\end{array}$ & $\begin{array}{l}\text { Chercheuse et colla- } \\
\text { boratrice du milieu } \\
\text { scolaire }\end{array}$ & $\begin{array}{l}\text { Collaborateur du } \\
\text { milieu scolaire }\end{array}$ \\
\hline Consentement des participantes & Chercheuse & Chercheuse & Ne s'applique pas \\
\hline $\begin{array}{l}\text { Travail préparatoire } \\
\text { Identification des difficultés des élèves } \\
\text { Identification des besoins des enseignantes }\end{array}$ & $\begin{array}{l}\text { Collaboratrices du milieu } \\
\text { scolaire } \\
\text { +enseignantes participantes }\end{array}$ & $\begin{array}{l}\text { Enseignantes partici- } \\
\text { pantes } \\
+ \text { collaboratrice }\end{array}$ & Ne s'applique pas \\
\hline $\begin{array}{l}\text { Expérimentation et diffusion } \\
\text { des résultats }\end{array}$ & $\begin{array}{l}\text { Enseignantes } \\
\text { Participantes }\end{array}$ & $\begin{array}{l}\text { Enseignantes } \\
\text { Participantes }\end{array}$ & Ne s’applique pas \\
\hline
\end{tabular}

Comme l'indique ce tableau, à l'exception de l'étape de consentement des enseignantes participantes - étape prise en charge uniquement par la chercheuse principale - , les collaboratrices du milieu scolaire de Pikogan ont activement participé à toutes les étapes du déroulement de la recherche. Elles ont d'ailleurs pris l'initiative, en accord avec la chercheuse principale, de donner l'information sur la recherche aux enseignants afin de solliciter leur participation. Ce n'est qu'une fois les enseignantes, désireuses de participer à la recherche, identifiées par les collaboratrices, que la chercheuse principale les a rencontrées pour compléter les informations sur les objectifs du projet et le plan anticipé de son déroulement, répondre à leurs questions et préoccupations concernant leur rôle et les rassurer quant aux exigences de la démarche de recherche. En ce qui concerne la collaboratrice de La Romaine, elle s'est jointe à l'équipe de recherche à l'étape de la demande de subvention, à l'invitation d'une cochercheuse qu'elle connaissait déjà. Comme les collaboratrices de Pikogan, elle a parti- 
cipé aux étapes subséquentes de la recherche. À la différence de Pikogan, une rencontre d'information sur la recherche et de sollicitation de participation a été organisée à La Romaine grâce au soutien logistique de la collaboratrice de cette communauté; ce qui a permis un rassemblement des enseignants des deux écoles et de la chercheuse principale. Cette rencontre a abouti au recrutement des enseignantes participantes qui a été confirmé ensuite par la collaboratrice. Dans ce contexte également, l'étape de consentement des participantes a été menée par la chercheuse principale seule. Le travail préparatoire d’identification des difficultés des élèves a été mené par les enseignantes, et celui d'identification des besoins de ces dernières par les collaboratrices ayant reçu le soutien des chercheuses.

Au-delà de ces deux contextes anicinape et innu, en contexte cri, le collaborateur identifié était un ancien étudiant de baccalauréat de la chercheuse principale. Malheureusement, la tentative de recrutement a avorté en raison du départ de celui-ci pour un nouveau poste dans une autre commission scolaire. Ainsi, trois collaboratrices incluant deux autochtones, six enseignantes dont deux enseignantes autochtones et leurs six classes de tous les cycles du primaire réparties dans trois écoles primaires des communautés anicinape de Pikogan et innue d'Unamen Shipu (La Romaine) ont participé à la recherche. Au cours de la réalisation du projet, une rencontre annuelle en présentiel de tous ces membres de l'équipe ainsi que de nombreuses rencontres de travail et de maintien de la collaboration à distance (la plupart du temps grâce à l'utilisation de technologies comme la visioconférence, la conférence téléphonique, etc.) ont eu lieu, entre chercheuses, entre chercheuses et collaboratrices, entre collaboratrices et enseignantes participantes, entre chercheuses et enseignantes participantes.

Bref, les trois témoignages du processus de recrutement montrent une démarche à la base de laquelle les préoccupations de recherche rejoignent ou répondent à des besoins de chacun des milieux scolaires concernés et où, de plus, une connaissance préalable des enseignantes participantes ou à tout le moins une rencontre en personne avec elles est nécessaire pour l'établissement de la recherche. Un autre point commun semble être une relation de confiance qui a servi de point d'ancrage à la recherche. La partie suivante porte d'ailleurs sur la définition de ce concept.

\section{Relation de confiance}

La relation de confiance s'exprime par le «sentiment de quelqu'un qui se fie entièrement à quelqu'un d'autre, à quelque chose» (Larousse, 2016, en ligne). Govier (1994) entend par cette relation une «attitude basée sur des croyances et des valeurs qui interpellent les sentiments et les comportements d'un individu» (Govier, 1994 tiré de Rousseau et coll., 2009 : 195). Quant à Rousseau, Deslandres et Fournier (2009 : 195), «la confiance est associée à un acte de foi à l'égard d'un étranger et elle présuppose un certain abandon aux décisions et aux actes d'autrui».

La littérature fait état de différents types de confiance. Comme illustré à la figure 1, Gagné et Mongeau (2011) en distinguent cinq, qu'ils positionnent sur un continuum allant d'un pôle négatif, caractérisé par la méfiance et le calcul des gains et des risques, à un pôle plus positif, fondé sur la confiance et un comportement non opportuniste. Plus près du pôle négatif se trouve la confiance dite rationnelle, basée principalement sur la finalité souhaitée ou le but à atteindre. La confiance organisationnelle serait, quant à elle, ancrée dans la structure ou l'institution représentée. Par exemple, un individu peut accorder une grande confiance à son banquier, non pas à l'individu en soi, mais à l'organisme qu'il représente. La confiance relationnelle s'appuierait sur une attitude coopérative entre les partis qui poursuivent des objectifs communs. Toujours selon ces auteurs, la confiance peut également être de type cognitif lorsqu'elle est basée sur le choix conscient de faire confiance à tel individu, 
pour telle raison, dans telle circonstance. Enfin, la confiance affective est celle qui est basée sur les émotions, sur «les qualités personnelles perçues chez l'individu à qui [on] accorde sa confiance» (p. 25). Bien que chaque niveau soit important dans l'établissement d'une relation de confiance, celle-ci devrait tendre vers le pôle positif de ce continuum pour assurer une collaboration optimale (Gagné et Mongeau, 2011).

Mettre ici Figure 1 : Continuum de la relation de confiance tirée de Gagné et Mongeau (2011 : 27) (voir document joint)

Figure 1 : Continuum de la relation de confiance tirée de Gagné et Mongeau (2011 : 27$)$

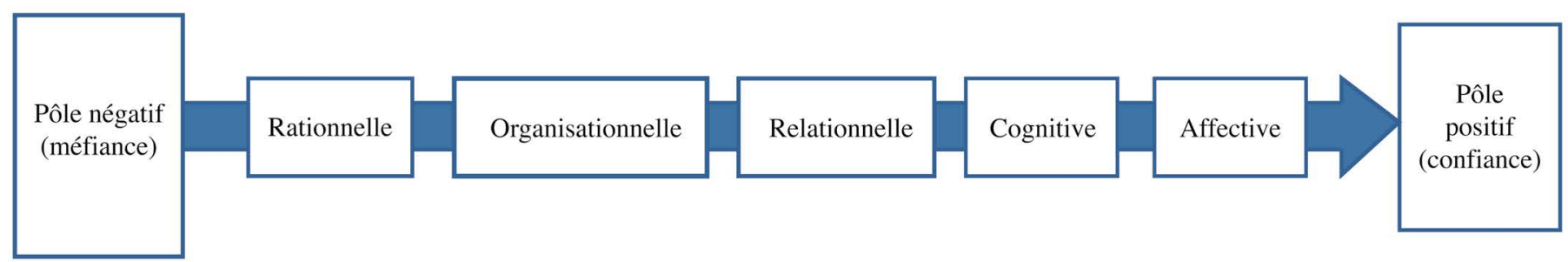

Jalava (2006) note quatre principes qui régissent le développement de la confiance : 1) il faut d'abord un engagement mutuel de deux participants, soit la personne qui fait confiance («truster») et la personne de confiance («trustee»), il s'agit là d’une condition sine qua non; 2) il faut également que les participants bénéficient d'une connaissance exacte de la situation et idéalement que ces derniers puissent se connaître mutuellement, ainsi «the building up of trust therefore depends on easily interpretable situations» (p. 22); 3), la confiance des autres ne se demande pas, elle peut seulement être offerte et être acceptée; et enfin, 4) la confiance doit être gagnée.

Cette relation de confiance à la base de nos expériences de recrutement et de recherche en contexte autochtone semble faire écho à des recommandations d'approche de recherche émanant du monde de la recherche autochtone. «ll importe de prendre le soin d'entretenir la relation du début à la fin d'un projet de recherche, et de maintenir un lien de confiance en vue de répondre aux besoins réels des Autochtones» (Blanchet-Cohen et Lainé, 2015 : 32).

\section{Recherche autochtone}

Dans son ouvrage Decolonizing methodologies Research and indigenous peoples, Linda Tuhiwai Smith (2012), chercheuse néo-zélandaise membre de deux tribus maories, professeure à l'Université Waikato (Nouvelle-Zélande), explore les complexités liées aux recherches en contexte indigène. Pour sortir du paradigme de la colonisation de la recherche, elle demande aux chercheurs d'adopter une position théorique locale qui permettra la création de connaissances cohérentes avec le cadre culturel. Smith les encourage à revoir les règles du jeu de la recherche afin de développer de nouvelles pratiques plus respectueuses, éthiques, sympathiques et utiles. 
Pour ce faire, elle propose que le cadre de recherche tienne toujours compte des concepts d'autodétermination, de décolonisation et de justice sociale. «The past, our stories, local and global, the present, our communities, cultures, languages and social practices-all may be spaces of marginalization, but they have also become spaces of resistance and hope» (p. 4).

De l'avis de Smith (2012), les recherches universitaires portent une orientation culturelle, un système de valeurs, une subjectivité, une conception du temps et de l'espace exprimés dans une forme de langage hautement spécialisé qui est étranger aux Autochtones. Ainsi, «des experts» parlent pour eux. L'auteure recommande donc aux Autochtones de remettre en question les motifs derrière les projets de recherche avant d'accepter d'y participer : À qui appartient cette recherche? Elle sert quels intérêts? Qui en bénéficiera? Qui l'écrira? Comment les résultats seront-ils publiés? Faire de la recherche autochtone, c'est adopter des approches qui visent à développer des moyens de permettre aux communautés de prendre des actions afin de résoudre des problèmes spécifiques. La recherche doit être amorcée en ayant comme postulat de base qu'il existe des solutions et que des réponses culturellement adaptées peuvent être générées. Un processus de consultation, de rencontres, de débats ouverts afin de prendre des décisions en équipe doit être à la base de la méthodologie adoptée. Les questions d'identité doivent être toujours centrales. Bref, autrement dit, n'entre pas dans ces milieux qui veut. La collaboration des communautés n'est possible que si les recherches sont menées de façon éthique et répondent aux préoccupations des communautés (Asselin et Basile, 2012; EPTC2, 2014; Desbiens, 2006). Elles doivent impérativement tenir compte des valeurs et des priorités des milieux et de leur culture.

De notre point de vue, certaines caractéristiques mises en œuvre dans les démarches de recherche collaborative et de recherche-action adoptées dans nos trois recherches répondent aux recommandations faites par ces auteurs. Nous expliquons ci-dessous en quoi ces deux formes de recherche respectent des principes du paradigme de recherche autochtone.

\section{Recherche collaborative ( $R C$ ) et recherche-action (RA)}

En sciences de l'éducation, la recherche collaborative est née d'un besoin de rapprocher les chercheurs et les praticiens tout en répondant à un besoin de développement professionnel des enseignants (Desgagné et coll., 2001). Desgagné (1997, 1998; Desgagné et coll., 2001) propose un modèle de recherche collaborative comportant trois étapes : la cosituation, la coopération et la coproduction. Dans l'étape de cosituation, le chercheur négocie avec les participants du projet ce qui sera objet de recherche et objet de formation. Dans l'étape de coopération, le chercheur met en place les conditions nécessaires pour réaliser une activité réflexive avec les participants. L'étape de coproduction correspond à celles des retombées de la recherche pour les participants et aux résultats de la recherche collaborative pour le chercheur. Dans ce type de recherche, le savoir se coconstruit dans l'activité réflexive et allie l'expertise du chercheur à celle des participants (Desgagné, 2001). En effet, ces derniers sont des acteurs compétents qui participent à la recherche et à l'activité réflexive mise en place par le chercheur. Quant à celui-ci, il met en place les conditions nécessaires pour s'assurer du bon déroulement de la recherche et de l'activité réflexive. Il est le principal acteur pour ce qui est des tâches formelles de la recherche, par exemple la recension des écrits scientifiques, le choix du cadre théorique, l'analyse de données, etc.

Quant à la recherche-action, une forme de recherche collaborative née d'un besoin spécifique du milieu de pratique de lier recherche et action, elle est menée dans une vision de changement de pratiques (Bourassa et Boudjaoui, 2012). Cette forme de recherche se déroule très souvent selon une démarche dynamique en spirale où il y a un retour entre l'action et la réflexion, la pratique et la théorie (Guay et Prud'Homme, 2011). Un des 
aspects qui caractérisent la recherche-action est l'implication constante et active des acteurs du milieu dans toutes les étapes du processus (Dolbec et Prud'homme, 2010). Les praticiens adoptent un rôle de cochercheurs alors que le chercheur joue plutôt un rôle d'accompagnateur pour faciliter le changement.

Bref, il va sans dire qu'un chercheur allochtone peut réaliser des recherches en milieu autochtone s'il partage la philosophie du paradigme de recherche autochtone (Wilson, 2013). D'ailleurs, l'historique des recherches en milieu autochtone a permis la révision des protocoles de recherche, incitant à la recherche collaborative et à la recherche-action (Éthier, 2010). En effet, ces deux formes de recherche respectent certains principes du paradigme de recherche autochtone. D’abord, la collaboration entre tous les acteurs est nécessaire pour mener à terme le projet. Le chercheur utilisant la RC ou la RA doit aussi tenir compte des besoins des participants. Dans les deux cas, les participants sont engagés et se mettent en action grâce aux activités que propose le chercheur durant le déroulement de la recherche. Les participants sont des acteurs clés de la recherche, leurs connaissances des besoins du milieu sont essentielles à l'avancement du projet; leurs savoirs sont considérés à part entière. Enfin, la RC et la RA ont des retombées concrètes pour les participants en termes de développement professionnel ou encore de matériel didactique pour les classes.

\section{Conclusion}

Des moments clés des trois expériences de recherche collaborative en contexte autochtone ont fait l'objet de cet article. Les aspects de chacun des projets reflétant une relation de confiance ont été soulignés. Ils ont par la suite été mis en rapport avec des éléments du paradigme de recherche autochtone afin de dégager leurs liens avec ceux de la recherche scientifique.

À la lumière de nos expériences, il nous apparaît évident qu'en contexte de recherche autochtone le recrutement des participants et la collaboration, sous-tendus par le consentement, vont de pair avec la relation de confiance qui doit, dès le départ, être établie entre tous les membres de l'équipe de recherche. En effet, nous pouvons retenir des témoignages rapportés des trois recherches qu'une connaissance des communautés et des participants concernés constitue un atout pour le recrutement. De plus, il a été important de prendre le temps de bien expliquer les objectifs, le fonctionnement anticipé du projet aux participants ainsi que leur implication, de répondre à leurs questions et préoccupations, de s'entendre sur ce que la communauté et eux-mêmes vont en tirer; de les considérer comme partenaires de recherche tout en définissant les rôles respectifs tout au long du déroulement de la recherche. Autrement dit, ces témoignages indiquent la présence des différents niveaux d'établissement d'une relation de confiance suggérés par Gagné et Mongeau (2011) : affectif, cognitif, relationnel, organisationnel, rationnel. Dans les trois cas, la relation de confiance établie semble être davantage orientée vers le pôle positif du continuum et avoir respecté les principes de son établissement (Jalava, 2006), à savoir : un engagement mutuel; une connaissance de la situation exacte; une confiance construite, gagnée.

Cette réflexion nous amène toutefois à interroger la procédure normale de l'éthique de la recherche, laquelle fait précéder le consentement au recrutement. En fait, dans nos expériences de recherche en contexte autochtone, nous avons dû, par la force des choses, explorer l'intérêt à la recherche de nos partenaires - et donc les possibilités de recrutement - généralement dès la conception de chacun des projets avant d'aller plus loin. En effet, quel serait l'intérêt de monter tout un projet sans partie prenante? 
Dans le 1er projet (Jacob, 2012-2017), les enseignantes qui ont exprimé leurs besoins de formation et de coin de jeu et qui ont vu le projet d'un bon œil ont été au départ des participantes potentielles au projet, en confiance avec la chercheuse, et ce, avant la démarche officielle de demande d'autorisation auprès du conseil de bande et le consentement à la recherche. Dans ce cas, la relation de confiance avait été établie, toutes partageaient des besoins communs et le consentement obtenu l'a été sur une base volontaire et dicté par la volonté des enseignantes de participer librement à la recherche.

Dans le 2e projet (Pellerin, 2011-2016), le recrutement des collaborateurs semble également aller de pair avec leur adhésion au projet, leur consentement libre et leurs actions pour recruter les participants au projet. Dans ce cas aussi, la relation de confiance était déjà en place et un besoin commun et partagé de tous a guidé tous les acteurs vers la planification et la réalisation du projet.

Dans le 3e projet (da Silveira, 2012-2015) également, même s'il a fallu attendre de recevoir l'autorisation du conseil de bande, les collaboratrices de Pikogan ont adhéré au projet, lequel coïncidait avec des problématiques de leur milieu, et ce, avant l'émission de la lettre d'intention. Elles ont joué un rôle d'éclaireur qui a été crucial dans l'interface des chercheuses avec le terrain de recherche, notamment dans l'adhésion et le recrutement des participantes au projet. Ici, rappelons que trois communautés ont été approchées pour participer au projet de recherche et que le collaborateur de l'une d'entre elles s'est désisté. Bien qu'une analyse rigoureuse n’ait pas été menée pour expliquer les raisons de ce désistement, nous constatons qu'il s'agit du seul milieu où la relation de confiance recherchée n'était pas présente avant le début du projet. Est-ce le fruit du hasard ou un épisode qui conforte notre prémisse voulant que la relation de confiance soit un ancrage incontournable en contexte de recherche autochtone?

En conclusion, notons que la recherche en contexte autochtone a longtemps subi une approche coloniale excluant toute reconnaissance des Autochtones de la part de chercheurs qui y travaillaient en terrain conquis. Ce passé a laissé des blessures profondes qui aident à comprendre les positions de certains chercheurs qui suggèrent entre autres de ne pas être un touriste-chercheurdans la communauté (Haig-Brown, 2001); «[ . . ] simply following Research Ethics Board processes does not guarantee ethical research 'on the ground' [... .]» (Tauri, 2014 : 143). 


\section{Bibliographie}

Asselin, H. et Basile S. (2012). Éthique de la recherche avec les peuples autochtones. Qu'en pensent les principaux intéressés? Éthique publique. Revue internationale d'éthique sociétale et gouvernementale, 14 (1).

Ball, J. (2009). Supporting Young Indigenous Children's Language Development in Canada: A Review of Research on Needs and Promising Practices, Canadian Modern Language Review, 66(1), 19-47.

Basile, S., Gentelet K. et Gros-Louis McHugh N. (dir.) (2015). Boîte à outils des principes de la recherche en contexte autochtone: éthique, respect, réciprocité, collaboration et culture. Assemblée des Premières Nations du Québec et du Labrador.

Blanchet-Cohen, N. et Lainé, A. (2015). Documenter le programme d’Aide aux devoirs : les éléments gagnants d'une recherche partenariale. Dans Gros-Louis Mchugh N., Gentelet K. et S. Basile (dir.), Boîte à outils des principes de la recherche en contexte autochtone : éthique, respect, réciprocité, collaboration et culture (p. 27-33). Assemblée des Premières Nations du Québec et du Labrador.

Bourassa, B. et Boudjaoui M. (2012). Des recherches collaboratives en sciences humaines et sociales (SHS). Enjeux, modalités et limites. Québec : Les Presses de l'Université Laval - Collection Pratiques d'accompagnement professionnel.

Conseil de recherches en sciences humaines du Canada (CRSH), Conseil de recherches en sciences naturelles et en génie du Canada (CRSNG), Instituts de recherche en Santé du Canada (IRSC). Énoncé de politique des trois Conseils : Éthique de la recherche avec des êtres humains, décembre 2014, repéré à http://www.ger.ethique.gc.ca/pdf/fra/eptc2-2014/EPTC_2_FINALE_Web.pdf

Cram, J. (1985). Northern teachers for northern schools: An Inuit teacher-training program. McGill Journal of Education/Revue des sciences de l'éducation de McGill, 20(2), p.113-131.

da Silveira, Y., Blaser, C., Moldoveanu, M. et Pellerin, G. et Maheux G. (2015). Exploration de nouvelles pratiques d'enseignement pour favoriser le développement de la compétence à écrire d'élèves anicinapek et innus du primaire. Rapport de recherche. Québec: FRQSC. http://www.frqsc.gouv.qc.ca

Desbiens, C. (2006). Du Nord au Sud : Géographie autochtone et humanisation du territoire québécois, Cahiers de géographie du Québec, 50(141), 393-401.

Desgagné, S. (2001). La recherche collaborative : nouvelle dynamique de recherche en éducation. Dans Anadón M. (dir.), Nouvelles dynamiques de recherche en éducation (p. 51-76), Québec : Les presses de l’Université Laval.

Desgagné, S. (1998). La position du chercheur en recherche collaborative : illustration de médiation entre culture universitaire et culture scolaire, Recherches qualitatives, 18, 77-105.

Desgagné, S. (1997). Le concept de recherche collaborative : l'idée d'un rapprochement entre chercheurs universitaires et praticiens enseignants, Revue des sciences de l'éducation, 23(2), 371-393.

Desgagné, S., Bednarz, N., Lebuis, P., Poirier, L. et Couture C. (2001). L'approche collaborative de recherche en éducation : un rapport nouveau à établir entre recherche et formation. Revue des sciences de l'éducation, 27(1), 33-64.

Dolbec, A. et L. Prud'homme (2009). La recherche-action. Dans Gauthier, B. (dir.). Recherche sociale: De la problématique à la collecte de données (p. 531-569), Sainte-Foy: Presses de l’Université du Québec.

Dorais, L.-J., (2004). Rectitude politique ou rectitude linguistique? Comment orthographier «Inuit» en français. Études/Inuit/Studies, 28 (1), 155-159 
Éthier, B. (2010). Terrain de recherche en milieu autochtone : la participation radicale du chercheur à l'ère de la recherche collaborative. Altérités, 2, 118-135.

Francis, N. et Reyhner, J.A. (2002). Language and Literacy Teaching for Indigenous Education: A Bilingual Approach. Clevedon, England: Multilingual Matters.

Gagné, A. M., et Mongeau P. (2011). La relation de confiance en relations publiques : vers un modèle d'adéquation contextuelle optimale. Communiquer. Revue de communication sociale et publique, 6, 23-40.

Guay, M.-H. et L. Prud'homme (2011). La recherche-action. Dans Karsenti T. et Savoie-Zajc L. (dir.), La recherche en éducation : étapes et approches (p. 183-211), Saint-Laurent, Les Éditions du Renouveau Pédagogique.

Haig-Brown, C. (2001). Continuing collaborative knowledge production: Knowing when, where, how and why, Journal of Intercultural Studies, 22(1), 19-32.

Jacob, E. (2017). Les rôles des enseignantes atikamekws à l'éducation préscolaire pour favoriser l'émergence de l'écrit en context de jeu symbolique: une recherche collaborative en milieu autochtone (thèse de doctorat, Université du Québec à Montréal, Montréal, Canada).

Jalava, J. (2006). Trust as a Decision. The Problems and Functions of Trust in Luhmannian Systems Theory, (academic dissertation, University of Helsinki).

Larousse, «Confiance», dans Dictionnaire de Français, [en ligne]. http://www.larousse.fr/dictionnaires/francais/confiance/18082 (Page consultée le 20 avril 2016).

Ministère de l'Éducation du Québec (2001). Programme de formation de l'école québécoise. Éducation préscolaire. Enseignement primaire. Québec: Gouvernement du Québec.

Pellerin, G. (2013). Mise en place d'une approche de support à la formation des enseignants inuit de Puvirnituq et d'Ivujivik à l'aide des TIC. Dans Maheux G. et R. Gauthier (dir.), La formation des enseignants inuit et des Premières Nations, problématiques et pistes d'action (p. 163-179), Québec, Presses de l'Université du Québec.

Rousseau, N., Deslandes, R. et H. Fournier (2009). La relation de confiance maître-élève : perception d'élèves ayant des difficultés scolaires, Revue des sciences de l'éducation de McGill, 44(2), 193-211.

Smith, L. T. (2012). Decolonizing methodologies: research and indigenous peoples (2 ${ }^{\text {nd }}$ ed.). London: New York, Zed Books. (1 édition en 1999).

Tauri, J. M. (2014). Resisting condescending research ethics in Aotearoa New Zealand, ALTERNATIVE, 10(2).

Wilson, S. (2013). Using Indigenist Research to Shape Our Future. Dans Grey, M., Coates, J., Yellow Bird M. and T. Hetherington (dir.), Decolonizing social work (p. 311-322), Farnham, Angleterre: Ashgate. 
TITRE: NAVIGATEURS, MILIEU DE LA FINANCE ET BÉNÉVOLAT : SOUVENIRS DE TERRAIN ET RÉFLEXIONS TRANSVERSALES SUR LE RECRUTEMENT

Auteur(s): BÉATRICE EYSERMANN

Publication: ReCRUTEMENT ET CONSENTEMENT À LA RECHERCHE : RÉALITÉS ET DÉFIS ÉTHIQUES

PAGES: $161-176$

ISBN: 978-2-7622-0357-8

Directeurs: Ana MARIN, BÉATRICE EySERMANn ET MiChel T. GIROUX

URI: HTTP://HDL.HANDLE.NET/11143/14116

DOI: HTTPS://DOI.ORG/10.17118/11143/14116 


\section{Navigateurs, milieu de la finance et bénévolat : souvenirs de terrain et réflexions transversales sur le recrutement}

Béatrice Eysermann, Ph. D., coordonnatrice du Comité d'éthique de la recherche sectoriel en santé des populations et première ligne, CIUSSS de la Capitale-Nationale.

Résumé : Ce texte propose une lecture transversale de trois terrains de recherche menés entre 1996 et 2003, en France et au Japon. Souhaitant travailler sur le temps social dominant, l'auteure a exploré trois contextes particuliers visant le temps vécu hors du temps social (navigateurs solitaires, France), le temps capitaliste (à travers celui des grands financiers, Japon), et le temps gratuit dans une société capitaliste (le bénévolat, France). Trois terrains et trois expériences de recrutement totalement différentes, qui ont posé des questions cruciales: Comment rencontrer des navigateurs solitaires? Comment contacter les grands financiers? Comment recruter lorsqu'on endosse un double rôle de bénévole et de chercheuse sur le terrain? La démarche intuitive de l'auteure sera en outre réfléchie sous le prisme de son expérience québécoise en éthique de la recherche.

Mots-clés : Temps, recrutement, éthique, anthropologie. 


\section{Introduction}

Cet article fait le bilan des trois terrains de recherche que j'ai menés dans le cadre de mon cursus universitaire en France, entre 1996 et 2003, auprès de navigateurs solitaires, de grands financiers et de bénévoles. Le recrutement de participants est la clé de tout projet de recherche, et l'idée de ce colloque m'a poussée à faire une lecture transversale de ces terrains sous l'angle du recrutement, alors exempt de toute forme normative d'éthique de la recherche, puisque les recherches en sciences sociales en France ne sont pas sujettes au passage devant un comité d'éthique pour approbation¹.

Il est important de souligner d'entrée de jeu que la méthodologie en matière d'ethnographie en France teinte le terrain et son approche de manière significative. Ainsi, telle qu'elle me fut enseignée, la recherche ethnographique se fait d'abord et avant tout sur le terrain, c'est-à-dire en faisant de l'observation participante, en droite ligne avec l'héritage de Malinowski et de ses Argonautes du Pacifique occidental (1922). Le principe est alors simple : plonger dans son terrain le plus vite possible sans s'être acculturé soi-même par le biais de lectures, d'hypothèses ou d'un cadre théorique, et cela dans le but de tout voir avec le moins de filtres possible. C'est ce que rappelle Althabe (1990), pour qui l'ethnographie se fonde sur ce qu'il appelle la «communication ordinaire» entre le chercheur et ses «sujets» plutôt que sur des règles méthodologiques strictes encadrant la collecte de données sur le terrain. C'est ce que rappellent également Auger et Colleyn (2004: 79) lorsqu'ils écrivent que «l'efficacité de l'enquête de terrain réside sans doute moins dans la recherche consciente et active que dans un apprentissage spontané». Ils ajoutent: «L'art du terrain, comme il a parfois été appelé, ne s'apprend pas dans les livres» (ibid.).

Ne pourrait-on pas déduire de ces quelques lignes que le terrain relèverait davantage d'un savoir-être et d'un savoir-faire modulables plutôt que de règles méthodologiques à appliquer? C'est ce que révèle la lecture transversale de mes trois terrains de recherche, dont le recrutement oscille entre adaptation, ajustement, réajustement, débrouillardise et parfois audace, afin de parvenir à recueillir le nectar de notre matière première : les données brutes.

1. Toutefois, les débats en la matière ressurgissent régulièrement. Voir par exemple en 2008, la revue Ethnographique.org pour son numéro L'éthique en anthropologie de la santé : conflits, pratiques, valeur heuristique (numéro 17, 2008), ou en 2014, la revue du Journal des anthropologues, numéro 136-137, Désirs d'éthique. Besoins de normes? 


\section{Une problématique générale, trois recherches}

J'avais, au moment de ma première recherche (1996), une fascination pour le thème du temps. Une fascination insaisissable, avec un besoin irrépressible de comprendre «le temps», ce métronome implacable qui rythme nos vies tout en étant une entité insaisissable et intangible, puisque le temps est une abstraction².

Une abstraction qui peut toutefois se saisir par la façon dont les individus la vivent et l'incarnent. C'est ainsi que j'orientai ma problématique vers le temps social dominant ${ }^{3}$, qu'incarne l'horloge à deux puis trois aiguilles, surtout depuis la première révolution industrielle qui l'a fait évoluer vers le temps capitaliste de l'accumulation ${ }^{4}$ (Weber, 1994).

J'ai alors imaginé un triptyque qui devait correspondre à deux mémoires (une maîtrise et un DEA5) et une thèse de doctorat pour comprendre le temps social dominant - capitaliste - de la société dans laquelle je vivais. J'ai choisi trois groupes d'individus qui reflétaient, selon moi, trois déclinaisons de ce temps dans trois manières d'y vivre, soit en tentant de s'en soustraire (navigateurs solitaires), soit en l'incarnant (grands financiers), soit en le contournant (bénévoles d'actions caritatives).

Plongeons maintenant au cœur des terrains et du recrutement des participants.

\section{Navigateurs solitaires (1996)}

Mon intérêt pour les marins est né de ce que ma famille et moi avions vécu comme étant les affres des familles de marin, ma sœur ayant été embauchée du jour au lendemain en Martinique sur des voiliers dits de «charter $^{6} \%$. C'est parce que je voulais comprendre cette vie particulière que, plusieurs années plus tard, ce sujet de recherche s'est imposé tout naturellement à moi lors du choix d'un sujet pour la maîtrise. Je cherchais alors à répondre à la question suivante : Pourquoi les marins partent sur l'eau, et pourquoi ils y retournent? (Eysermann, 1997)

2. Alain Leroi-Gourhan (1967) y consacrera un chapitre dans son ouvrage Le geste et la parole (vol. 2). Pour lui, le temps fait partie des symboles de la société, au même titre que l'espace, les attitudes et le langage, ou l'esthétisme.

3. Parmi les caractéristiques de ce temps social dominant, on trouve celle de régulateur d'ordre social, d'horloge sociale, de donneuse de temps (Sue, 1995 ; Rifkin, 1996), de métronome pour l'organisation des rythmes collectifs (Sue, 1995) et pour le changement de rythme social (Nowotny, 1994). Dans ma thèse de doctorat, j'écrivais : "parce qu'il est partagé et utilisé par tous les membres de la société, il est le temps social dominant» (Eysermann, 2011 : 25).

4. Voir toute la démonstration dans la thèse, chapitre I: «Redéfinition du bénévolat dans le champ conceptuel de la linéarisation du temps, ou comment définir le temps «libre»» (Eysermann, 2011).

5. Diplôme d'études approfondies (DEA), équivalent à la première année du $3^{e}$ cycle et qui se solde par l'écriture d'un mémoire de recherche. Ce diplôme a été remplacé par le master à finalité de recherche en 2005.

6. Voiliers appartenant à des propriétaires qui engagent des équipages afin de pouvoir voyager avec leur famille ou leurs amis. Ces voiliers, avec équipage, peuvent aussi être loués à des touristes. 


\section{La préparation du terrain}

Pour préparer le terrain, j'ai lu les livres de marins bien connus comme Slocum (1990) ou Moitessier (1986), et j'étais totalement fascinée par ce que je découvrais en termes de voyage et de liberté. La version rédigée de leurs journaux de bord donne la sensation de voyager avec eux. Le profil des participants s'est donc d'abord défini à travers mes lectures : je souhaitais trouver ces navigateurs solitaires «à la Moitessier», marginaux de la société, vivant sur la mer et les océans.

J'ai aussi rassemblé les souvenirs de tout ce que ma sœur m'avait raconté sur la vie de marin pour parfaire ma préparation. Je savais ainsi que beaucoup vivaient en Bretagne, notamment un de ses amis, Pilou, avec qui elle avait traversé l'Atlantique. Mon but était de rejoindre le village de Bretagne où je trouverais cet homme et de côtoyer le plus de marins possible dans leur milieu. Je savais aussi que les marins étaient très hospitaliers, je pensais donc n'avoir aucun mal à trouver asile sur leur bateau pendant mon séjour. J'avais aussi arboré ce que je pensais être une tenue de circonstance - une veste de quart ${ }^{7}$ et un jean noir — dans l'idée de susciter, bien humblement, une reconnaissance identitaire visuelle chez les marins. J'étais prête. Je pris alors un train de nuit pour La Trinité-sur-Mer, où j'arrivai aux premières heures du matin. Nous étions en mars. J'avais deux semaines devant moi pour faire mon terrain.

\section{Premiers pas sur le terrain}

Dès mon arrivée, je confiai ma valise à un pêcheur du marché aux poissons. J'avais jusqu'à midi pour trouver asile sur un bateau et commencer mon terrain. Une fois les mains libres, je fis un tour dans les deux magasins d’accastillage 8 que j'avais repérés, pensant alors y trouver le profil de marins qui m’intéressait.

Après avoir écouté ma brève présentation, le vendeur du premier magasin me dit ne connaitre que des marins qui faisaient de la course en solitaire, et pas ces marins «à la Moitessier» que je recherchais. Un peu déçue, je me dirigeai vers le second magasin où je trouvai cette fois le type de marin souhaité : un homme qui préparait son bateau pour la traversée de l'Atlantique en solitaire. Ce premier informateur m'offrit l'hospitalité pour deux nuits sur son bateau, et je pouvais revenir lui confier ma valise pour le reste de la journée.

Comme je n'avais connu que des marins qui fumaient, le bar-tabac du village situé juste en face des quais me semblait être une place de choix pour établir mon «quartier général», y voir les allées et venues, faire la connaissance des marins et assurer une présence continue sur les lieux. Je décidai que j’y viendrais pour rédiger mon carnet de terrain et relire mes notes. C'est comme cela que, le soir de mon arrivée, mon premier informateur m’a présentée, moi et ma recherche, à des membres d'équipage de voilier qui repartaient dès le lendemain sur leur bateau. L'un d'eux me prêta son studio pour les deux semaines suivantes, en plus d'un vélo. J'étais prête à faire mon terrain.

\footnotetext{
7. Un «quart» est une période de temps pendant laquelle un marin est à la barre de son bateau. On appelle «veste de quart» le vêtement caractéristique que portent les marins en mer et qui comporte notamment des anneaux en métal pour pouvoir s'accrocher au bateau.
}

8. Magasin spécialisé dans la vente de matériel pour les bateaux. 
En parlant à ceux que je côtoyais jour après jour, il devenait alors évident que trouver tout un corpus de marins «à la Moitessier» serait impossible dans ce village : la voile étant un sport très onéreux, les marins doivent trouver des commanditaires et donc faire des courses pour publiciser leurs noms. Ce fait apparaissait d'autant plus évident que - et je le savais - les bateaux de course portent justement le nom de leur commanditaire? De fait, le profil de mes participants changea : je cherchais désormais des marins ayant déjà fait des courses à la voile en solitaire avec le soutien financier d'un ou de plusieurs commanditaires.

Je retournai donc au premier magasin d'accastillage pour obtenir le nom de quelques-uns. J'avais par ailleurs de la chance : une transat ${ }^{10}$ allait avoir lieu trois semaines plus tard et plusieurs marins se trouvaient déjà sur place avec leur maxi, des bateaux de 25 mètres. II semblait alors que j'arriverais plus facilement à trouver mon corpus.

II me fallait aussi partir en quête de ce Pilou que ma sœur avait connu. De fil en aiguille, je parvins à trouver une piste: on m'indiqua une maison dans laquelle se trouvait quelqu'un qui pourrait me renseigner. Une fois rendue, je me suis présentée en mentionnant l'objet de ma visite. Posté sur le seuil de la porte, l'homme me questionnait, relançait mes réponses, revenait sur plusieurs, et je sentais que tout pouvait basculer d'un moment à l'autre : j'avais en effet la certitude que, dans ce village où tout le monde se connaît, le moindre impair ou la moindre impatience de ma part pouvait me coûter la suite de mon terrain... C'est alors au bout de dix longues minutes que l'homme me tendit finalement la main pour me dire: «C'est moi, Pilou». Et c'est avec lui, et avec une célébrité de la voile des années 1980, que j'ai fait non seulement des entrevues, mais aussi, et de manière aussi impromptue qu'inespérée, un tour en bateau de quelques heures dans le froid mordant du petit vent de nord-est bien connu des Bretons.

Au final, ma présence continue sur le terrain a facilité mon intégration auprès d'un milieu qui semblait m'avoir adoptée. Tout le monde a fini par savoir qui j'étais sans m'avoir pour autant rencontrée, confirmant alors mon sentiment d'être observée. Ainsi, il m’est arrivé plusieurs fois d'être interpellée dans la rue par ces mots «Hé l'étudiante, si tu veux, je suis libre pour te parler», tandis que je ne restais jamais seule plus de dix minutes dans le bar-tabac. En effet, il y avait toujours quelqu'un qui venait s'asseoir à ma table pour me raconter la vie de marin, soit parce qu'il en était un, soit parce qu'il en connaissait un. J'ai même gardé le souvenir d'une femme, accotée au comptoir, qui m’a interpellée alors que je me rendais à la salle de bain. Elle m'a tout raconté de ce qu'elle vivait dans le monde de ces hommes de la mer, mais je n'avais ni crayon, ni papier, ni magnétophone pour l'enregistrer. Au terme de notre heure de discussion, je me précipitai sur mon carnet de terrain pour noter tout ce dont je me rappelais. Contre toute attente, cette entrevue informelle a beaucoup servi à l'analyse.

9. Par exemple, en 2017 : le trimaran Macif (compagnie d'assurance française) sur lequel François Gabart établit un nouveau record de tour du monde en solitaire ; le trimaran Banque Populaire sur lequel Francis Joyon et son équipage remportent le Record du Trophée Jules Verne; Peter Burling remporte la coupe de l'America avec son équipage sur le trimaran Emirates Team New Zealand (pour Fly Emirates).

10. Abréviation de «transatlantique»: course de bateau qui vise à traverser l'Atlantique, en solitaire ou en équipage. Chaque course a un nom spécifique. Il s’agit ici de la course Spi-Ouest France. 
La clé du recrutement a été le contexte dans lequel il prenait corps. Et il est un fait indéniable : plus je m'imprégnais du terrain, plus je saisissais les systèmes de représentation jusqu'à les vivre intérieurement. Ainsi, au fil des jours, je commençais à ressentir cet insondable fossé entre «marins» et «terriens» dont tous me parlaient; je me souviens même de m'être sentie prisonnière de la terre et d'avoir eu une furieuse envie de naviguer. Peutêtre l'aurais-je fait si je les avais côtoyés plus longtemps?

Je découvrais alors que comprendre l'univers des marins c'était aussi une manière de l'intégrer et, in fine, la passerelle la plus directe, pour moi la plus naturelle, vers le recrutement des participants. C'est comme cela qu'en deux semaines, outre mes carnets de terrain, j'ai effectué 17 entrevues formelles, plusieurs informelles, et côtoyé tous les profils de marins en présence : marins pêcheurs, marins en équipage, marins de course et un marin solitaire hors course. Ma stratégie de recrutement se dessinait clairement vers l'imprégnation du terrain, et vers la confiance que je saurais inspirer.

C'était mon premier terrain. C'est celui qui m’a le plus appris. C'est celui que j'ai le plus aimé.

\section{Grands financiers (1997)}

Un concours de circonstances a fait que je me trouvais au Japon lorsquej'ai fait ma recherche pour mon second mémoire ${ }^{11}$ en France. Si le temps des navigateurs solitaires en dehors de la société était celui que je nommais «le temps des choses et des éléments», j'entrepris de comprendre le temps capitaliste à travers ceux qui l'incarnaient dans les salles de marché ou trading floor. Comme je l'écrivais en introduction de mon mémoire, «je voulais me plonger dans un monde où la culture du temps était à ce point fondamentale qu'elle en devenait aliénante» (Eysermann, 1998: 3).

\section{Préparation du terrain}

Pour préparer le terrain, j'ai lu quelques livres sur les financiers pour me familiariser avec leur vocabulaire et repris l'étude de l'anglais, sachant que je devrais faire des entrevues en français et en anglais. J'ai également regardé une bonne dizaine de fois le film Wall Street d'Oliver Stone (1987) pour m'imprégner de l'atmosphère du monde de la finance et pour pratiquer mon anglais. Ma préparation dura environ deux mois. Je voulais travailler sur le stock exchange, c.-à-d. la bourse, qui, pour moi, «se jouait telle une pièce de théâtre dont les acteurs ne pensaient qu'à leur montre» (Eysermann, 1998: 3).

11. Voir note 5. 
Je partis ainsi un après-midi entier à la Bourse de Tokyo, la Tokyo Stock Exchange (TSE), pour trouver deux éléments qui m’ont forcée tout de suite à changer de lieu de recrutement. Le premier élément, pourtant prévisible, était de n'y voir que des travailleurs japonais. Le second élément, inattendu, était le suivant : je n'étais pas face à des acteurs se bousculant dans tous les sens pour réaliser des transactions d'achat et de vente, mais face à des hommes, apparemment paisibles, travaillant pour une bourse totalement électronique, et où il ne régnait pas, à ma grande déception, ni confusion, ni bruits, ni émulations. Et à 15 heures, tout s'est arrêté, c'était la fin des heures de marché au pays. Je ne trouverais pas là les informateurs dont j'avais besoin.

Ce n'est alors pas tant le profil des participants que le lieu d'exercice de leur profession qui changea. Les quelques banquiers avec qui j'avais discuté m'avaient parlé de deux catégories de travailleurs: les traders donnant les ordres d'achats et de ventes, et les brokers qui les exécutent. Mon observation de la TSE m'avait dissuadée de poursuivre dans cette voie, et j'optai alors pour les salles de marché des banques internationales pour y trouver des traders et des brokers francophones ou anglophones, faisant ou ayant fait du trading ou du brokerage, tout type de marchés confondus ${ }^{12}$.

Mais déterminer son terrain de recherche ne signifie pas que celui-ci est facile d'accès. C'est en me rendant au siège social d'une de ces banques internationales que je me suis retrouvée devant une véritable forteresse aux portes vitrées dont l'accès était de toute évidence réservé aux seuls privilégiés qui en possédaient le code. Inutile d'envisager de taper pour se faire ouvrir; il fallait trouver une stratégie pour rendre légitime ma présence sur les lieux.

\section{Élaborer une stratégie de recrutement adaptée au contexte}

En réfléchissant au milieu de la finance et à ses contraintes en termes de temps, je décidai d'une approche par contact direct et à distance. Pour cela, je choisis des noms au hasard dans l'annuaire de la Chambre de commerce française de Tokyo ${ }^{13}$, et rédigeai une lettre de présentation en français et en anglais que j'envoyai par télécopie. Cette lettre contenait les grandes lignes de mon projet, la promesse de confidentialité et une demande de participation à une entrevue pour m'aider dans ma recherche. J'y précisais que je les appellerais dans les deux jours pour sonder leur intérêt et leur laissais mes coordonnées téléphoniques.

L'idée d'une lettre de présentation envoyée par télécopie avait plusieurs atouts. J'ai vite compris que les moyens de communication rapides étaient la manière la plus sûre de capter leur attention et que, parmi ceux existant à cette époque, le fax était sans doute le meilleur. Cela permettait aussi de contourner plusieurs obstacles importants: se soustraire à une présentation trop longue par téléphone, et de la rallonger dans un anglais approximatif, leur faire perdre du temps pendant les heures de marché - ce qui m’aurait assuré un refus irréversible voire une fermeture du terrain. Sans compter que - je l'appris plus tard - le téléphone était l'outil de sollicitation continuel auquel les grands financiers étaient exposés, ce qui ajoutait à la pertinence de ne l'utiliser qu'à leur demande.

En plus des noms pris dans l'annuaire de la Chambre de commerce, je demandai à chaque participant de me donner le nom d'un ou deux grands financiers, et je procédais toujours par fax.

12. Exemples de types de marché : actions, obligations, devises étrangères, etc.

13. Qui venait de l'Ambassade de France et référençait les entreprises françaises de la capitale. 
Cette stratégie a eu 99 \% de succès; une seule personne a refusé. Un financier m’avait même téléphoné en me disant: «Bon, j'ai votre fax sur mon bureau depuis trois jours. Alors, vous voulez m'interroger ou pas?» J'ai évidemment pris rendez-vous avec cette personne, même si mon agenda était plein, car les plages de temps pour une entrevue devaient se situer en dehors des heures de marché14 et en dehors des heures de travail (qui dépendaient en outre des résultats des marchés). Trois plages étaient plus favorables : entre 11 heures et midi, après 17 heures et après 19 heures.

Mais avoir un rendez-vous ne signifiait pas pour autant que le recrutement était effectif. Les rendez-vous étaient parfois reportés jusqu'à quatre ou même cinq fois en raison d'impératifs dus aux fluctuations du marché, de rendez-vous de dernière minute avec des clients ou d'autres empêchements. Je devais ainsi confirmer chaque rendez-vous deux jours avant, le matin, et une heure avant, et commençais toujours la conversation, qui durait tout au plus quelques secondes, en demandant si c'était le bon moment pour téléphoner.

\section{Observations non préméditées}

Mon terrain m'a en outre permis de m'imprégner plus directement de l'atmosphère du monde de la finance par d'autres éléments imprévus. J'ai eu ainsi le privilège, par un de mes informateurs, d'aller observer une salle de marché de brokers pendant un après-midi entier. Les autres personnes présentes ignoraient totalement qui j'étais et la raison de ma présence. Une ambiance lourde pesait dans la salle. Dans l'introduction de mon mémoire, j'ai écrit:

Un des brokers a pris de vitesse un de ses collègues. Après quelques éclats de voix sans conséquence, chacune des deux parties s'est contenue et n'a plus rien dit, laissant place au calme de l'effusion des marchés, qui ne cessa jamais, sous le regard attentif de leur manager (1998: 7).

Cet extrait est révélateur de toute une stratégie interne orientée vers la division pour le profit que j'analysais en détail dans le corps de mon mémoire. Mais, pour l'heure, j’en étais repartie avec un terrible mal de tête.

J'ai eu l'opportunité également d'observer de près la relation de pouvoir très marquée entre trader et broker en étant conviée par un trader à rencontrer «son» broker dans l'un des restaurants les plus huppés de la ville. Selon ses termes, il fallait que je voie comment son broker allait «s'écraser devant lui», en ajoutant: «ll faut que vous voyez ça». Ainsi, sans qu'il n'ait jamais été informé de ma venue, et sans y avoir jamais consenti, ce broker est devenu mon hôte et paya la totalité de la facture. Il va sans dire qu'il a eu par la suite l'obligation d'accepter une entrevue - qui n'a duré ensuite que les quelques minutes qu'il lui a fallu pour se défaire de cette obligation, ce que je n'ai jamais dit au trader qui s'était bien sûr enquis du déroulement de ladite entrevue.

14. Dépendamment des marchés que les financiers suivaient. Rares étaient ceux qui se limitaient aux marchés de l'Asie. Beaucoup travaillaient aussi sur les marchés européens et américains, ce qui signifie qu'en tenant compte du décalage horaire ils pouvaient travailler jusqu'à tard le soir ou même la nuit. 
La stratégie utilisée pour approcher mes informateurs est empreinte de la compréhension d'un contexte de travail particulier dans lequel l'ethnographie par le porte-à-porte n'aurait rien donné. Il fallait s'ajuster. Par ailleurs, la confidentialité nécessaire et promise à mes informateurs exigeait que personne dans leur salle de marché ne soit au courant qu’ils allaient «parler». Et ce, pour deux raisons. La première est liée bien sûr à l'enquête de terrain elle-même. Mais la seconde illustre pleinement la réalité de ce terrain : à cet endroit, plus qu'ailleurs peut-être, l'information, c'est de l'argent. Ainsi, la moindre fuite produit un impact immédiat sur les marchés, sans parler d'un potentiel délit d'initié.

Au fil de mes quatre mois d'entrevues et d'observations, j'étais donc susceptible de recueillir des informations secrètes, et, si la confidentialité était toujours de mise, c'est sur le capital confiance qu'il leur fallait miser à mon endroit. Comme précédemment, c'est lors des entrevues que je tissais le lien de confiance, en démontrant notamment une compréhension exempte de tout jugement de leur univers. Si le travail de l'anthropologue n'est pas de juger, il peut parfois être confronté à des valeurs qui ne sont pas les siennes, voire en être dérouté. Et quoique ce fût mon cas, jamais je ne l'ai laissé paraître; j'interprétais toujours en fonction du contexte et de leur univers, renforçant ainsi le lien de confiance qui prêtait souvent à confidences. C'est ce lien qui m'a ensuite permis d'être invitée à faire des observations en terrain privé (repas au restaurant) ou, même, interdit (salle de marché), et d'obtenir d'autres noms.

\section{Bénévoles (1999-2002)}

Mes deux premiers terrains m'avaient permis de comprendre le temps dans deux univers opposés : le temps présent des choses et des éléments des marins solitaires devenait celui de l'instantanéité et de la simultanéité chez les grands financiers. Il me restait à comprendre le temps qu'on donne (gratuitement) dans un univers où on le vend, et c'est tout naturellement que je m'orientai cette fois vers les bénévoles. Mais lesquels choisir?

Je décidai de faire ma recherche à Marseille, en France, et commençai par chercher une association où faire mon terrain. Le travail commença par l'épluchage du registre des quelque 800 associations marseillaises, toutes vocations confondues. J'éliminai les associations pour les animaux maltraités (sensibilité personnelle), de même que les sports et loisirs, pour me concentrer sur les associations à vocation humanitaire, instaurées sur des bases laïques ou religieuses. À cela s'ajoute une typologie particulière à la France : les associations constituées en vertu de la loi $1901^{15}$, dont la légitimité est reconnue; et celles qui ne le sont pas et donc risquaient de disparaître durant ma recherche.

Ma première intention était de comparer les deux associations dites humanitaires que j’avais sélectionnées : une, militante, à vocation de faire sortir les gens de la misère par la culture et l'éducation; et l'autre, qui s'inscrit dans la tradition de la charité et du don. Après un essai dans chacune de ces associations, je décidai de ne me concentrer que sur celle fondée sur la tradition chrétienne de charité.

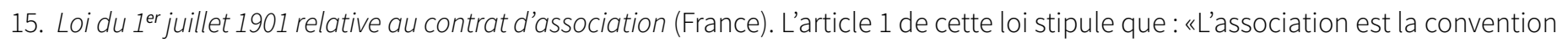
par laquelle deux ou plusieurs personnes mettent en commun, d'une façon permanente, leurs connaissances ou leur activité dans un but autre que de partager des bénéfices. Elle est régie, quant à sa validité, par les principes généraux du droit applicables aux contrats et obligations.» Les associations constituées en vertu de cette loi ont un statut pérenne. Pour plus d'informations, voir le lien suivant: https://www.legifrance.gouv.fr/affichTexte.do?cidTexte=LEGITEXT000006069570 


\section{Commencer par de l'observation participante}

Lors de ma première visite à l'association, après avoir expliqué mon vaste objet d'étude, «le bénévolat», la secrétaire m'a orientée vers l'accueil de nuit : «ll y a une activité avec les sans-abri ce soir. Si vous voulez, venez.» J'ai immédiatement accepté, démontrant par là même, pensais-je, mon intérêt et déjà, mon engagement. Dès le premier soir, je distribuais des soupes à des sans-abri, ce qui laissa en moi la trace indélébile de ce premier souvenir : un homme mangeant une boîte pour chats. Après m'être ressaisie (le sans-abri avait-il vraiment, en plus, besoin de mes larmes), cette première impression : approcher les sans-abri était d'une facilité déconcertante. L'observation participante commençait.

Au fil des deux ans de terrain, et de ma présence constante à l'association, j'ai probablement vécu toute la palette des émotions possibles, que ce soit au contact des sans-abri ou avec les bénévoles qui parfois me testaient. J'ai participé à toutes les (très) nombreuses réunions organisées par l'association, assuré la coordination de l'activité de nuit pendant deux mois et participé à l'évaluation de cette activité pour un organisme externe.

\section{Recruter en terrain connu?}

Le recrutement des participants était loin d'aller de soi : tout le monde faisait fi de mon identité d'étudiante-chercheuse pour ne me considérer que comme simple bénévole. Je me suis alors inspirée de la stratégie de recrutement des grands financiers pour me distancer du terrain et revêtir le chapeau d'anthropologue - que je n'avais pour ma part jamais quitté.

Je rédigeai une lettre titrée «une anthropologue parmi vous» dans laquelle j'expliquais ma présence à l'association, mon terrain, et que j'allais procéder à l'étape des entrevues. J'y décrivais les critères de confidentialité et de liberté de participation, et leur demandais de me contacter. Les neuf salariés et quelques bénévoles ont accepté de m'accorder une entrevue, ce qui s'inscrivait tout à fait dans la vocation du rendre service associatif. Mais les choses avaient changé : tous me considéraient à présent à travers mon «désengagement» bénévole (Fillieule et Broca, 2001).

Je soulignerai ici un élément intéressant: à ma grande déception, les entrevues que je faisais ne m’apprenaient rien de plus que mes observations profanes. Souffrais-je d’acculturation? Les bénévoles démontraient quant à eux un humanisme et un altruisme parfois en contradiction avec mes observations de terrain ${ }^{16}$. Ce n'est que plus tard, une fois sortie du terrain et en analysant les données, que je saisis toute l'ampleur et l'envergure du temps associatif, en apparence flottant et insaisissable, de même que le niveau d'acculturation qui fut le mien au bout de deux années consécutives.

Pour clore mon terrain, j'ai également voulu recruter des sans-abri, ce que je fis, par grand vent, un soir froid de janvier. Je me suis présentée comme une «petite étudiante» qui faisait un travail pour l'Université, et je posai mes questions aux trois sans-abri qui se tenaient là. L'intervenant qui m'accompagnait devait assurer une présence discrète et muette, mais il ne s'y tint pas et posa des questions pour son travail. Cela a quelque peu court-circuité mon entrevue informelle, mais cela fait aussi partie du terrain.

16. Les exemples de contradiction sont nombreux. Je me souviens notamment du discours humaniste très senti d'une bénévole envers les sans-abri, alors qu'avant de partir en tournée de nuit, cette dernière s'évertuait à cacher les denrées sous les sacs ou les containers car, disait-elle, ils allaient les voler. Chose que je n'ai pour ma part jamais observée. 
À la différence des deux premiers terrains, ma présence continue sur les lieux pendant une période de deux ans faisait de moi une bénévole comme les autres. Les acteurs en présence étaient d'ailleurs bien plus intéressés par mon engagement indéfectible que par le vrai but de ma présence, du moins dans les premiers temps. Au début, je me présentais toujours aux bénévoles et dans toutes les réunions comme une étudiante de doctorat qui faisait du bénévolat pour sa recherche. Cela ne semblait intéresser personne. Je le fis ensuite de façon plus sporadique, puis j'ai arrêté.

Le défi, plus tard, fut de changer d'identité à leurs yeux pour redevenir ce que je n'avais jamais cessé d'être: une doctorante sur son terrain de recherche. Si le lien de confiance m'a permis de recruter des participants sur les deux premiers terrains, rappeler aux bénévoles la (véritable) raison de ma présence a semblé parfois ternir ou émousser ce lien : j'étais parfois prise à partie et devais me repositionner souvent pour les convaincre de ma neutralité (Eysermann, 2010). J'avais compris qu'il y avait un moment clé à ne pas dépasser pour recruter et faire les entrevues, etj'ai respecté ce moment. L'étape suivante ne pouvait être que mon retrait de l'association, et nous le savions tous.

Quand j'y suis retournée en 2003 pour valider mon analyse, le temps avait fait son œuvre, et je fus accueillie comme une vieille amie «qui a fait sa thèse sur l'accueil de nuit». J'ai pu même bénéficier d'un passe-droit pour intégrer une équipe de tournée de nuit le soir même sans passer par le nouveau planning organisationnel qui avait été mis en place dans l'intervalle.

\section{Réflexions transversales sur le recrutement}

Mes trois terrains de recherche ont en commun la familiarisation des contextes par la lecture et tout autre élément dont je pouvais disposer. C'est ce qui me donna suffisamment confiance pour entrer en contact avec le terrain et savoir comment faire mon recrutement. Ce fut néanmoins très différent d'un terrain à l'autre.

Pour les marins, nous avions, mes parents et moi, toutes nos années d'expérience comme famille de marins, et ma sœur nous en avait présenté plusieurs. Je pensais en comprendre la mentalité, et je ne m'étais pas trompée. Le fait d'avoir une sœur qui a navigué dans ce milieu m'a ouvert aussi les portes de ce monde. J'ai approfondi cette connaissance profane et familiale par la lecture de magazines de voiles et de ces carnets de bord des grands marins solitaires. Ce qui m'a très vite permis de m'intégrer à leur monde et de collecter beaucoup de données pour mon analyse.

Pour ce qui est des financiers, ma brève préparation m’a permis de comprendre le contexte temporel dans lequel ils baignaient douze à quinze heures par jour, à savoir que le temps c'est de l'argent et qu'il faut faire de l'argent. En réponse, je n'avais d'autre choix que d'orchestrer le recrutement autour de l'injonction de ne surtout pas leur faire perdre de temps. Il fallait donc ruser et créer le moyen le plus astucieux de les motiver à participer à ma recherche. La clé du recrutement était là.

Je n'avais jamais fait de bénévolat humanitaire avant d'entrer dans l'association pour y faire mon terrain. L'observation participante a débuté dès le premier soir avec les bénévoles auprès des sans-abri, et ensuite quotidiennement dans la sphère associative où je passais mes journées. Ma présence continue pendant deux ans aura été autant un formidable vecteur pour collecter des données qu'elle n'est devenue un défi lorsqu'il fallait recruter en terrain connu. Car, et il faut le souligner, devenir bénévole pour ma thèse m'avait forgé, malgré moi, 
une double identité dont il me fut impossible de me départir sans conséquence : après les entrevues, j’ai bien senti que je n'étais plus vraiment la bienvenue. Une pause était nécessaire, et il fallait la respecter.

Ces contextes distincts mettent en relief l'aspect intuitif du terrain, tant il faut savoir sentir, jauger, juger de tous les éléments à disposition pour saisir le bon moment pour recruter. Et j’ai pu constater que c'est cette connaissance de leur milieu qui inspirait aussi confiance aux participants potentiels, qui réciproquement m'ont aidé à enrichir ma collecte de données : bien des marins se sont assis à ma table dans le bar-tabac du coin pour me parler de leur vie; j'ai fait de l'observation dans une salle de marchés (interdite) et dans un restaurant; les bénévoles, eux, me faisaient confiance à travers mon engagement sans limites, et m'appréciaient d'abord pour cet engagement.

Donc ma stratégie a été dans tous les cas d'inspirer confiance, d'en savoir assez pour que les personnes présentes me prennent au sérieux et de ne pas en savoir trop, ce qui risquerait de compromettre la raison d'être de ma présence; en effet, c'est à eux de m'aider à comprendre leur univers. Faire de l'observation participante m'a aussi permis d'avoir les mêmes référents que mes participants lors des entrevues en plus de pouvoir truffer mes mémoires ou ma thèse d'exemples vécus.

\section{Pour conclure : Passer outre un comité d'éthique de la recherche (CER)?}

Je suis tout naturellement obligée de terminer mon texte en parlant du comité d'éthique de la recherche, puisqu'aucune de ces recherches n'a fait l'objet d'une évaluation en ce sens.

En prenant appui sur mes neuf années passées à la coordination d'un CER à Québec, je ferai trois constats :

1. Jamais je n'aurais pu deviner le déroulement de mes terrains et moins encore le décrire dans un protocole bien ficelé pour qu'il soit évalué adéquatement. Les profils théoriques de marins et de grands financiers que je recherchais se sont révélés caducs dès mon arrivée sur le terrain. J'aurais dû faire des amendements en cours de route, ici depuis la Bretagne ou le Japon, et attendre le feu vert. Si les CER demandent beaucoup de précision dans les protocoles, peut-être que certaines circonstances exigent au contraire plus de souplesse eu égard aux imprévus de terrain et de ses acteurs.

2. Pour ce qui est du recrutement en passant par des tiers, comme les CER ont tendance à le prôner pour toutes les recherches, j'ai pu en bénéficier, mais pas de manière systématique, et seulement sur l'invitation expresse d'informateurs voulant m'aider. C'est ainsi que les marins ont parfois servi d'intermédiaires suivant leur propre initiative ou m'interpellaient directement dans la rue (supra). Pour les financiers, je présume que j'aurais écrit dans mon protocole que je passerais par les réceptionnistes, avec un échec assuré à la clé. Comment les aurais-je approchées puisque je ne pouvais pas entrer dans ces forteresses vitrées? Qui l'aurait fait pour moi? Il aurait fallu justifier de la pertinence d'un contact direct et à distance, qui relevait bien plus d'une compréhension intuitive du contexte que de données théoriques. 
3. Plusieurs données ont été collectées spontanément dans des contextes imprévus. Pour en rappeler quelques-uns : lors de la navigation avec deux marins, de l'observation d'une salle de marché et dans le restaurant; des différents rôles que j'ai endossés à l'association (bénévole, responsable de l'activité, évaluatrice externe), et de l'entrevue avec les sans-abri - qui a presque tourné en entrevue professionnelle pour l'intervenant qui m'accompagnait. À bien y réfléchir, aucun de ces exemples n'était prévisible : ils émanaient des participants eux-mêmes, qui attendaient mon acquiescement immédiat. C'est aussi là, entendons-nous, toute la beauté du terrain. Aurais-je profité d'autant de spontanéité si j'avais dû tout prévoir, ou tout faire approuver avant de donner une réponse? Si l'éthique de la recherche donne des balises solides, le chercheur pourrait aussi, dans de telles circonstances, jauger du niveau de risque, faire parler son expérience et prendre les bonnes décisions. Sur mes terrains, avec le recul, je pense pouvoir dire que c'est ce que je fis, et j'en savoure encore la richesse aujourd'hui.

Alors, pourrait-on se passer des CER? Non. En neuf ans dans un CER, j'ai plusieurs fois été soulagée que des protocoles soient ainsi évalués, car ils nécessitaient une sévère révision au plan éthique. Par contre, il ne faut pas penser ce processus en sens unique, ce qui est trop souvent le cas : les chercheurs doivent parler et faire valoir leur contexte de recherche puisque ce sont les seuls à le connaître vraiment. Les chercheurs, certains, font fausse route quand ils cherchent à répondre ce qu'ils supposent que le CER veut entendre au lieu de mordre dans leur connaissance du contexte. Ce qui donne d'ailleurs des mauvaises réponses, vagues, creuses. Et on les repère tout de suite. Je n'ai pas vu beaucoup de recherches pour lesquelles les chercheurs ou les étudiants ne connaissaient rien de leur terrain. J'ai même déjà vu les membres du CER changer leur position suite à l'argumentaire de chercheurs qui explicitaient les raisons pour lesquelles ils ne pouvaient pas souscrire à une de leurs recommandations.

Alors ainsi, pour les recherches en sciences sociales du moins, les chercheurs et les étudiants ont la responsabilité d'élaborer leur protocole au plus précis de leur connaissance de terrain afin de viser une saine et profitable collaboration plutôt que de construire - de part et d'autre, d'ailleurs - des lignes d'incompréhension. Pourquoi? Parce que le recrutement est, la plupart du temps, du moins pour les sciences sociales, le point phare de l'évaluation éthique des CER, avant même le consentement. Le dialogue ne peut alors que gagner à être éclairé et ouvert, encore une fois, de part et d'autre.

\section{Annexe : résultats de recherche ou l'immanence du temps présent}

Mes recherches m'ont appris que le temps social dominant se muait inévitablement vers une seule temporalité : le présent. C'est celui du temps des choses et des éléments chez les marins, qui ne revêt que $10 \%$ du temps de voyage, qui les pousse à retourner sans cesse sur les mers et les océans. Ce temps présent où ils se sentent en harmonie avec leur univers et libres de tout, de tous, où ils savourent un instant après l'autre.

Si les marins savourent ce temps qu'ils gagnent pendant leur voyage, les financiers sont prisonniers du temps capitaliste. Rivés sur le temps de l'accumulation pour le futur, ils sont pris au piège de la temporalité des marchés dont ils ne savent s'extraire : le temps présent de l'instantanéité ou de la simultanéité des transactions d'achats et de vente. Je n'ai rencontré que des millionnaires sur mon terrain. Ils m'ont tous dit que le premier million est le premier objectif, mais qu'il est très vite atteint. Ainsi, pour garder les pieds sur terre, ils ne pensent pas en termes de millions, mais d'unité : «On a un, on veut deux», etc. Dans cet univers capitaliste par excellence, ils se sentent contraints de travailler de 15 à 18 heures par jour; l'un d'eux m'a même dit qu'il aimerait ne jamais dormir. Serviteurs de ce temps de l'accumulation, se déclarant incapables de décrocher ou de prendre des vacances afin de pouvoir surveiller sans cesse les fluctuations des marchés et leurs positions, ces millionnaires vivent le plus absurde des paradoxes : ils n’ont pas le temps de dépenser leur argent... 
Quant aux bénévoles, je me suis posé la question du temps gratuit et de sa signification dans un univers où on le vend. À défaut d'accumuler des millions, ce sont les heures que les bénévoles accumulent, tant leur temps devient élastique pour les salariés de l'association qui l'étirent sans cesse pour en repousser les limites. Mais la surprise fut sans doute de constater que, réciproquement, les salariés sont d'insoupçonnés donneurs de temps. Les bénévoles ne se cachaient pas pour affubler les salariés du paradoxe selon lequel percevoir un salaire et avoir bon cœur sont incompatibles. De fait, les bénévoles faisaient des salariés de vrais prestataires dévoués exclusivement à leur service, et n'avaient de cesse d'user et d'abuser de ce privilège qu'ils se donnaient, les empêchant ainsi de faire le travail pour lequel ils étaient réellement payés. Tous les salariés se voyaient alors contraints de faire des heures supplémentaires, le soir ou les fins de semaine. Gratuitement cette fois. Les tensions entre bénévoles et salariés cohabitaient avec l'obligatoire bonne entente et légèreté qui régnaient toujours à l'association.

Ma conclusion fut celle-ci : le temps des bénévoles comme celui des salariés est élastique, et aucune des deux catégories d'acteurs n'a conscience du temps qui lui est donné. Ceci crée une incompréhension temporelle, source de tensions et de malentendus, qui ne se résolvent jamais. Car, si le temps gratuit est invisible aux yeux de celui qui le reçoit, le corollaire est d'être extensible à l'infini ou de ne jamais suffire. Et les temps s'étirent. Et la boucle recommence.

Trois contextes, trois temps présents, toujours accolés au temps social dominant auquel, quoi qu'on fasse, on ne peut se soustraire. J'ai voulu à travers ces recherches comprendre aussi bien le temps de ma société que ma fascination pour ce thème. Si j'ai l'impression d'avoir répondu à ma question, ma fascination reste entière. 


\section{Bibliographie}

Althabe, G. (1990). Ethnologie du contemporain et enquête de terrain, Terrain, 14, 126-131. DOI : 10.4000/terrain.2976

Augé M. et Colleyn J.-P. (2004). L'anthropologie, Chapitre III. Le terrain. Paris : PUF.

Eysermann B. (2011). L'ouvre des donneurs de temps dans notre société. Bénévoles et salariés de l'humanitaire dans une association caritative marseillaise, Éditions universitaires européennes.

Eysermann B. (2010). Un terrain dans une association caritative marseillaise (France) à l'épreuve de l'éthique de la recherche (Canada). Éthique, méthodologie et responsabilité, Éthique publique, 12 (1), 179-200. DOI: 10.4000/ethiquepublique.185

Eysermann B. (1998). Trader/broker versus capitalisme: vers un temps de la postmodernité? (Mémoire de DEA, Université d'Aix-Marseille I, France).

Eysermann B. (1997). Vivre le temps présent. La navigation à voiles en solitaires, (mémoire, Université d'Aix-Marseille I, France).

Fillieule, O. et Broqua C. (2001). Désengagement et conséquences biographiques de l'engagement. Dans Actions associatives, solidarités et territoires (p. 157-162). Saint-Étienne (France) : Presses universitaires de Saint-Étienne.

Leroi-Gourhan A. (1967) Le geste et la parole, Albin Michel, vol 2.

Malinowski. (1922), Les Argonautes du Pacifique occidental

Moitessier, B. (1986). La longue route, Arthaud.

Nowotny, H. (1994). Time. The Modern and Postmodern Experience, traduit de l'allemand par Neville Price, Polity Press.

Rifkin, J. (1996). La fin du travail, La découverte.

Slocum, J. (1990). Navigateur en solitaire, Babel-Actes Sud.

Sue, R. (1995). Entre travail et temps libre : l'émergence d'un secteur quaternaire. Cahiers internationaux de sociologie, 99, 401-415.

Weber, M. (1994). L'éthique protestante et l'esprit du capitalisme, Plon, Pocket.

\section{Numéros de revue}

L'éthique en anthropologie de la santé : conflits, pratiques, valeur heuristique, 17, 2008 : http://www.ethnographiques.org/Numero-17-novembre-2008

Désirs d'éthique. Besoins de normes?, 136-137, 2014: https://jda.revues.org/656 
TITRE: ANALYSE RÉTROSPECTIVE DE L'UTILISATION DES RÉSEAUX SOCIAUX POUR LE RECRUTEMENT D'ADOLESCENT(E)S ATTEINTS DE NF1, DE FEMMES ENCEINTES, DE CHERCHEURS/EUSES FONDAMENTALISTES ET DE MÉDECINS

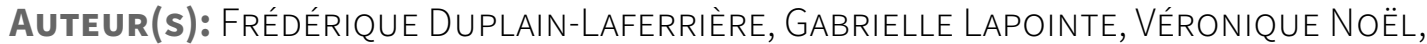
GAËL BOUFFARD, CHANTAL BOUFFARD

PUBLICATION: RECRUTEMENT ET CONSENTEMENT À LA RECHERCHE : RÉALITÉS ET DÉFIS ÉTHIQUES

PAGES: $177-202$

ISBN: 978-2-7622-0357-8

Directeurs: Ana MARIn, BÉATrice Eysermann et Michel T. GIROUX

URI: HTTP://HDL.HANDLE.NET/11143/14117

DOI: HTTPS://DOI.ORG/10.17118/11143/14117 


\section{Analyse rétrospective de l'utilisation des réseaux sociaux pour le recrutement d'adolescent(e)s atteints de NF1, de femmes enceintes, de chercheurs/euses fondamentalistes et de médecins}

Frédérique Duplain-Laferrière( ${ }^{(a)}$, Gabrielle Lapointe ${ }^{(a)}$, Véronique Noël(a) Gaël Bouffard $^{(a-b)}$, Chantal Bouffard(a)

a - Laboratoire de recherche transdisciplinaire en médecines, génétique et sciences sociales, Service de génétique, Département de Pédiatrie, Faculté de Médecine et Sciences de la Santé, Université de Sherbrooke, Québec, Canada;

b - Gaël Bouffard Services-conseils, Sherbrooke, Québec, Canada

Mots clés : recrutement en ligne, recherche qualitative, éthique, réseaux sociaux

Résumé : Dans le cadre de la recherche qualitative, lorsque les problématiques portent sur des sujets sensibles ou s'intéressent à des populations restreintes, le recrutement est une étape cruciale qui peut s'avérer difficile. Dans certaines conditions, les réseaux sociaux peuvent représenter une solution intéressante pour optimiser le recrutement. Cette publication présente trois expériences de recrutement avec les réseaux sociaux Facebook, LinkedIn et ResearchGate, lors de trois projets de recherche qualitative et transdisciplinaire sur : (1) les perceptions et les préoccupations d'adolescent(e)s canadien(ne)s concernant les impacts de la neurofibromatose de type 1 (NF1) - une condition génétique pouvant avoir de nombreuses manifestations cliniques (physiques, neurologiques, psychologiques, psychosociales) - dans leurvie sociale et privée; (2) les besoins et les points de vue de femmes enceintes estriennes concernant les nouveaux tests génomiques prénataux; (3) les préoccupations de chercheurs/euses et médecins quant aux effets de l'intégration chromosomique de l'herpès virus humain de type 6 sur la santé. Pour chaque cas, nous abordons les particularités des réseaux sociaux que nous avons utilisés, nos expériences de recrutement, les contextes dans lesquels les difficultés de recrutement ont été rencontrées, les solutions apportées, les avantages et les inconvénients perçus. Avant de conclure, nous discuterons aussi des dimensions éthiques de l'utilisation des réseaux sociaux pour le recrutement à la recherche et des défis à relever par rapport à l'équité dans la représentativité et dans l'accès au bénéfice de recherche, à la confidentialité et à la protection de la vie privée, au respect de la personne et de l'intégrité de ses identités, ainsi qu'à l'importance d'une approche collaborative et co-constructive avec les comités d'éthique de la recherche. 


\section{Introduction}

Le recrutement est une étape particulièrement cruciale de la recherche. Non seulement il est une des principales conditions de la faisabilité d'une étude, mais la composition de l'échantillon qu'il permettra de constituer aura des répercussions sur la qualité des données ainsi que sur la validité scientifique et la transférabilité des résultats. La réussite d'un projet de recherche dépend donc en grande partie de la capacité de l'équipe de recherche à rejoindre et à intéresser les populations ciblées.

Dans le contexte de la recherche qualitative, plusieurs facteurs peuvent complexifier l'étape du recrutement. Entre autres, le niveau de difficulté peutêtre plus élevé lorsque les études portent sur des sujets délicats, intimes ou à la marge de la légalité, ou lorsqu'elles s'adressent à des populations restreintes avec des caractéristiques particulières. Il peut aussi s'avérer difficile de recruter des personnes mineur(e)s, des patient(e)s atteint(e)s de maladies graves, des professionnel(le)s surchargé(e)s de travail ou des individus dispersés à travers le monde.

Pour ce qui nous concerne, nous avons rencontré des problèmes de recrutement lorsque les problématiques auxquelles nous nous intéressions portaient sur des maladies génétiques graves, le diagnostic prénatal pendant la grossesse ou les points de vue des professionnel(le)s de santé et des chercheurs/euses concernant les conditions de prestation des services de santé. Toutefois, lorsque les contextes et la spécificité des études s'y prêtent, l'utilisation des réseaux sociaux peut devenir une solution intéressante pour optimiser le recrutement.

En fait, dans le cadre de recherches qualitatives en santé, ce chapitre partage trois expériences de recrutement réalisées à l'aide des réseaux sociaux Facebook, Linkedln et ResearchGate, où les méthodes traditionnelles ne se sont pas avérées efficaces (tableau 1). Ces études, menées par des étudiant(e)s et des membres du Laboratoire de recherche transdisciplinaire en médecines, génétique et sciences sociales, portaient sur des problématiques qui leur était spécifiques, qui recouraient à des approches méthodologiques distinctes et qui s'adressaient à des populations différentes: adolescent(e)s atteint(e)s de la neurofibromatose de type 1 (NF1), femmes enceintes, chercheurs/euses et médecins intéressés par l'intégration chromosomique de l'herpès virus humain de type 6 (ciHHV-6) (tableau 1). La première partie de cette contribution fait un survol des principales particularités des réseaux sociaux que nous avons utilisés. Les trois parties suivantes présentent les trois projets de recherche pour lesquels nous avons utilisé le recrutement en ligne. Pour chaque étude, nous exposons brièvement: les caractéristiques du projet de recherche, les contextes dans lesquels les difficultés de recrutement ont été rencontrées, les solutions adoptées, ainsi que les avantages et les inconvénients perçus par l'équipe de recherche et les questions suscitées par ces derniers. Avant de conclure, la cinquième partie discute de notre expérience de recrutement en ligne en tenant compte de certaines dimensions éthiques relatives à l'utilisation des réseaux sociaux. 


\section{Particularités des réseaux sociaux}

Les avantages et les inconvénients du recrutement en ligne sont en grande partie liés aux caractéristiques des réseaux utilisés. Dans le cas qui nous intéresse, il s’agit de Facebook, Linkedln et ResearchGate.

\section{Facebook}

Avec 1,28 milliard d'utilisateurs/trices par jour, Facebook est le réseau social le plus populaire au monde (Facebook, 2017). Pour en faire partie, il faut ouvrir un compte Facebook et créer un profil d'utilisateur/trice. Ce dernier permet de regrouper des informations qui nous concernent, des photos, divers contenus se rapportant à notre vie sociale et privée, ainsi que nos préférences dans toutes sortes de domaines. Chaque utilisateur/trice dispose aussi d'un journal personnel servant à publier des commentaires, des humeurs, des vœux, des textes, des images, des photos, des vidéos, des fichiers et divers documents. Un compte Facebook permet aussi de se faire des ami(e)s Facebook, de créer des pages ou des groupes Facebook, ou encore, d'y participer. II donne aussi accès à plusieurs fonctionnalités et à une grande variété d’applications.

À partir de son compte Facebook, un membre peut envoyer ou recevoir des demandes d'amitié et devenir ami(e) Facebook avec d'autres membres du réseau. Toute demande d'amitié doit nécessairement être acceptée, refusée ou ignorée. Il est impossible de devenir ami(e) avec quelqu'un sans son consentement. Les ami(e) s Facebook peuvent échanger par messagerie privée et par vidéoconférence. II est aussi possible d'écrire des commentaires et de partager des publications sur le journal de nos ami(e)s Facebook ou d'autres membres de la communauté, s'ils le permettent. Certaines applications comme Twitter ou Instagram peuvent être associées au profil d'utilisateur/trice.

La page d'accueil d'un compte Facebook se présente comme un fil d'actualité où défile une liste continuellement actualisée de notre profil, de notre journal et de nos publications, ainsi que ceux de nos ami(e)s. S'y affichent aussi des publicités ciblées selon nos intérêts et nos activités, ainsi que les publications des pages Facebook que nous aimons et des groupes Facebook auxquels nous appartenons selon le niveau de confidentialité souhaité. Par exemple, le contenu d'un compte et d'un profil peut être accessible à tous les membres de la communauté Facebook, limité aux ami(e)s Facebook ou encore réduit à certains d'entre eux. Seule la photo du profil est accessible pour tous les internautes, même pour ceux qui ne sont pas membres de Facebook. Il est toutefois possible de bloquer l'accès à la photo.

Comme mentionné plus haut, les membres du réseau ont l'opportunité de créer une page Facebook. À la différence des profils d'utilisateur/trices, ces pages sont $100 \%$ publiques et peuvent être administrées par plus d'une personne. Elles portent généralement sur un sujet ou un thème particulier et servent à donner de la visibilité aux entreprises, aux organisations ou aux individus. Les pages Facebook contribuent à la formation de communautés web. Tous les internautes, avec ou sans compte Facebook, peuvent avoir accès au contenu d'une page Facebook à partir du web, mais, par contre, les personnes jugées indésirables peuvent être bloquées. Une page Facebook ne permet pas d'entretenir des liens d'amitié, mais les membres de la communauté peuvent signaler qu'ils aiment son contenu. Dès lors, ce dernier apparaît dans leur fil d'actualité. Ainsi, leurs ami(e)s Facebook pourront à leur tour aimer la page, la commenter ou la partager. Enfin, les administrateurs/ trices d'une page Facebook peuvent aussi payer pour qu'une publication, leur page ou un site web puisse atteindre un auditoire plus grand ou plus ciblé sous forme de publication commanditée (Kosinski et coll., 2015). 
Les groupes Facebook peuvent aussi être créés et administrés par une ou plusieurs personnes. Les membres du groupe peuvent cependant y publier du contenu et commenter les publications des autres membres, ce qui parfois donne lieu à des discussions. Ce format s'apparente davantage à un forum. Les groupes Facebook sont de trois ordres : 1) publics, où tout le monde peut voir le contenu sans être membre; 2) privés, où seuls les membres ont accès au contenu - toute demande pour rejoindre un groupe privé doit être approuvée par un(e) administrateur/trice; 3) secrets, où les membres doivent obligatoirement être invité(e)s par l'administrateur/ trice ou d'autres membres. Un tel groupe n'apparaît pas dans les résultats d'une recherche sur Facebook ou sur un moteur de recherche.

\section{LinkedIn et ResearchGate}

À la différence de Facebook, les réseaux sociaux comme Linkedln et ResearchGate sont à usages professionnels. La finalité de LinkedIn, par exemple, est de développer et d'entretenir des contacts professionnels. Ce réseau compte plus de 433 millions de membres dans plus de 200 pays (Linkedln, 2017). Chaque membre a un compte personnel où son cheminement de carrière, ses expertises et ses réalisations sont mis en valeur. Sur LinkedIn, les membres du réseau ont accès aux contenus de tous les comptes. Les utilisateurs/trices peuvent partager des contenus et des publications qui apparaîtront sur le fil d'actualité des gens faisant partie de leurs réseaux de contacts. Les membres utilisent cette plateforme principalement pour mettre de l'avant leurs expériences de carrière, chercher de l'emploi et partager leurs opinions professionnelles.

ResearchGate est aussi un réseau professionnel, mais il se distingue de Linkedln du fait qu'il est axé sur les carrières scientifiques. Avec plus de 10 millions de membres (ResearchGate, 2017), il met en contact des scientifiques de partout dans le monde, facilitant les échanges d'expertises et de connaissances. Entre autres, les utilisateurs/trices y partagent leurs articles scientifiques et peuvent interroger d'autres membres, afin d'obtenir des informations ou de résoudre certains des problèmes rencontrés pendant un projet de recherche.

Linkedln et ResearchGate sont tous deux dotés d'une messagerie privée, semblable à une boîte de courriel, permettant des contacts formels et professionnels entre les utilisateurs/trices. Ils se distancent ainsi de réseaux comme Facebook, où les communications privées entre les membres s'apparentent plus à une messagerie instantanée.

\section{Expériences de recrutement en ligne - Projet No. 1 : Perceptions d'ado- lescent(e)s canadien(ne)s atteint(e)s de neurofibromatose de type 1}

\section{Caractéristiques du projet de recherche}

La première expérience de recrutement en ligne présentée ici est une recherche qualitative réalisée au Canada avec des adolescent(e)s de 15 à 19 ans ayant reçu un diagnostic de NF1. II fait partie d'une recherche ethnographique plus vaste menée auprès des associations canadiennes de neurofibromatose. L’objectif général de l'étude était de connaître les besoins et les préoccupations des adolescent(e)s atteint(e)s concernant les impacts de la NF1 sur leur santé, leur vie sociale et leur vie privée. Il est utile de préciser ici que la NF1 est une maladie génétique autosomale dominante, dont l'apparition est à $50 \%$ due à une transmission héréditaire et à $50 \%$ due à une mutation spontanée. Elle peut avoir des impacts médicaux, esthétiques et liés à l'apprentissage, auxquels s'ajoutent des problèmes psychosociaux induits par l'environnement social (figure 1). Les 
personnes atteintes présentent un risque élevé de stigmatisation, de rejet et de dépression. Ce qui est aggravé du fait qu'il est impossible de prévoir l'évolution ou la gravité de la NF1.

\section{Contexte dans lequel les difficultés de recrutement ont été rencontrées}

Avec le consentement des parents, nous avons commencé à mener des entrevues semi-dirigées auprès des jeunes afin de connaître : ce qu'ils avaient besoin de savoir sur les aspects médicaux et génétiques de la NF1, ainsi que sur ses modes de transmission ou d'apparition; les impacts de la NF1 dans leur vie sociale et privée; leurs perceptions de la pertinence de la communication intergénérationnelle face aux difficultés entraînées par cette condition génétique.

Malgré les bonnes relations que nous avions développées avec ces jeunes au fil des années et leur désir que nous réalisions cette recherche, ils devenaient peu collaboratifs et sur la défensive lors de l'entrevue. Nous leur avons rapidement demandé s'ils/elles étaient à l'aise de participer à l'étude, et ces adolescent(e)s ont répondu qu'ils/elles avaient accepté de participer à l'entrevue pour faire plaisir à leurs parents. En fait, aucun(e) ne se sentait à l'aise pour répondre à des questions portant sur des sujets intimes comme la sexualité et la qualité de leurs relations avec leurs parents, avec des adultes qui, en plus d'être chercheurs/euses, connaissaient leurs parents.

Devant cette évidence, nous avons changé de stratégie pour une approche de recherche collaborative intégrant les adolescent(e)s dans la détermination des objectifs de recherche et dans la conception des outils de cueillette des données. Ensemble, nous avons convenu d'opter pour une entrevue écrite, asynchrone, en ligne ${ }^{1}$ (Ward, 1999). Cette technique combine un questionnaire en ligne composé principalement de questions semi-ouvertes et une messagerie qui permet aux chercheurs/euses et aux participant(e)s d'échanger et d'interagir à des moments qui leur conviennent (Ross et coll., 2005). Pour les adolescent(e)s, il était aussi essentiel de donner eux-mêmes leur consentement sans l'intervention de leurs parents.

Après avoir obtenu l'accord du Comité d'éthique de la recherche (CER) du Centre de recherche clinique du Centre hospitalier de l'Université de Sherbrooke (CRCHUS)², nous avons fait une deuxième tentative de recrutement en partenariat avec les associations canadiennes de NF1 et avec l'aide des médecins spécialistes de la NF1 qui collaborent au projet. Toutefois, la plupart des personnes atteintes de NF1 ne sont pas membres d'une association. Lorsqu'elles le sont, il s'agit le plus souvent d'adultes. On y retrouve très peu d'adolescent(e) s. Par conséquent, ce mode de recrutement restreignait la population accessible et très peu de jeunes ont répondu à l'appel. Ensuite, considérant que les symptômes de la NF1 peuvent être de nature évolutive, il arrive que les adolescent(e)s n'aient pas besoin d'un suivi médical serré. Les médecins ne pouvaient donc pas les informer du projet et les recruter. Il fallait trouver une autre solution pour atteindre le plus grand nombre de participant(e)s possible.

1. L'entrevue écrite asynchrone en ligne (EEAL) est une technique de recueil des données qualitative. Elle débute avec une série de questions ouvertes auxquelles les participant(e)s répondent en ligne, par écrit. Elle se distingue du questionnaire du fait que les participant(e)s et les chercheurs/euses peuvent ensuite échanger par messagerie.

2. Les approbations éthiques des projets dont il est question dans cet article ont été accordées par le CER du CRCHUS, désigné aujourd'hui par CER du CIUSSS de l'Estrie-CHUS. 


\section{Solution apportée}

Dans ce contexte, Facebook semblait une alternative prometteuse puisque ce réseau social est particulièrement populaire chez les jeunes de cette tranche d'âge (Lenhart et coll., 2015). Pour notre troisième tentative de recrutement, une assistante de recherche a été chargée de la réalisation de cette étape du projet ${ }^{3}$. Elle a créé un compte Facebook à l'usage exclusif du projet de recherche, ce qui permettait d'en contrôler le contenu. En empruntant le style «article de blog», nous y avons publié une invitation à participer à la recherche accompagnée d'une note informative visant à expliquer le projet. Un lien hypertexte intégré à la note menait directement au site d'hébergement du formulaire d'information et de consentement en ligne. C'est en cliquant sur le bouton qui permettait d'accepter de participer à la recherche que les adolescent(e)s avaient accès à l'entrevue en ligne. Comme le compte était en mode public, l'ensemble des participant(e)s potentiels(le)s sur la toile, ami Facebook ou non, pouvait avoir accès à l'information concernant le projet de recherche.

Être détenteur d'un compte Facebook permettait d'agir en tant que personne sur ce réseau social, avec tous les avantages en découlant tels qu'«aimer» les pages en lien avec la NF1, rechercher des pages à «suivre», ou encore, faire des demandes d'amitié avec des acteurs/trices important(e)s de la communauté NF1 (administrateurs/trices de groupes, associations, individus atteints, groupes de soutien, etc.) et accepter celles qui nous étaient adressées. Toute personne qui le désirait pouvait ensuite partager nos publications, dont l'information nécessaire pour participer au projet de recherche. Nous avons recherché les pages et les groupes Facebook susceptibles de rejoindre des jeunes canadien(ne)s de 15 à 19 ans inclusivement ayant reçu un diagnostic de NF1. Pour maximiser le succès du recrutement, nous avions établi certains critères d'inclusion et d'exclusion. D'abord, la page ou le groupe devait être en activité, c'est-à-dire qu'on pouvait y retrouver au moins une nouvelle publication au cours du dernier mois. D'une façon arbitraire, nous avons fixé un seuil minimum de 100 membres ou abonnés pour maximiser la dissémination. Les pages ou les groupes devaient être en français ou en anglais. Comme cette étude concerne les adolescent(e)s canadien(ne)s, les pages et groupes provenant de l'extérieur du Canada étaient exclus d'emblée. Nous avons cependant fait une exception pour les pages ou les groupes de France, parce que beaucoup de Canadien(ne)s français(e)s semblaient y être abonné(e)s. Finalement, le sujet devait être en lien avec la NF ou la NF1 (soutien, informations, médical, rencontre, général, etc.).

Nous nous sommes abonnés à toutes les pages Facebook correspondant à ces critères, pour un total de 27 pages. Nous avons aussi rejoint 28 groupes Facebook. L'assistante de recherche a ensuite contacté les administrateurs/trices par messagerie privée pour leur expliquer le projet et leur demander de publier les informations sur l'étude ainsi que le lien hypertexte permettant d'y participer sur leur page Facebook ou, pour ce qui concerne les groupes, la permission de les partager. Lorsque les groupes étaient très actifs, nous repartagions notre annonce chaque semaine, ou aux deux semaines, pour demeurer visibles dans leur fil d'actualités.

Pour la page Facebook de notre projet, afin de nous assurer la meilleure visibilité possible, nous avons suivi les techniques les plus efficaces de l'optimisation du référencement par les moteurs de recherche entre autres en adaptant notre contenu à l'aide de mots clés ciblés.

3. F. À cette étape, Frédérique Duplain-Laferrière, doctorante au Laboratoire de recherche transdisciplinaire en médecines, génétique et sciences sociales, a été engagée comme assistante de recherche pour le projet sur la NF1. 
Utiliser Facebook pour le recrutement de cette recherche en ligne s'est avéré très avantageux. Premièrement, nous avons augmenté d'une façon significative nos chances de recruter des individus qui ne fréquentaient pas les associations de neurofibromatose. Deuxièmement, cette approche a permis d'accéder aux adolescent(e) s qui ne pouvaient pas être approché(e)s par les médecins collaborateurs pendant la période de recrutement. Un troisième avantage était que l'anonymat était parfaitement respecté. Comme la sollicitation n'était pas directement dirigée vers une personne et qu'il n'y avait pas de lien entre notre formulaire de consentement et le questionnaire, nous ne pouvions savoir qui participait à la recherche, contrairement à ce qui se passe en contexte associatif. Enfin, le fait d’avoir un compte d'utilisateur créé spécialement pour cette étude a permis d'éviter d'ouvrir une porte sur la vie privée de la chercheuse et de conserver un contexte professionnel.

De plus, l'accès aux groupes permettait de lire des propos qui, sans être utilisés dans la recherche en cours, offraient une meilleure compréhension du contexte. D’ailleurs, Facebook est de plus en plus reconnu par les chercheurs/euses pour explorer les comportements propres à des contextes particuliers (Wilson et coll., 2012).

\section{Inconvénients perçus}

Le cas de cette recherche montre bien que nous aurions tort de penser que le recrutement en ligne, et particulièrement par Facebook, est plus facile que les modes de recrutement conventionnels. II faut d'abord créer un compte, une page et une note informative avec le design approprié pour le mode de recrutement et le type de participant(e)s. Il faut ensuite rejoindre les administrateurs/trices et les convaincre du bien-fondé de la recherche. Il est aussi important de comprendre que, dans le contexte d'études en ligne, les participant(e) s peuvent quitter la recherche en un seul clic. II est donc primordial de rendre son expérience intéressante en s'assurant d'un design et d'un contenu de qualité (Kosinski et coll., 2015), y compris lors de la phase du recrutement. Il faut ensuite consacrer beaucoup de temps pour assurer une bonne présence sur les pages et les groupes, ce qui permettra de créer, de partager des publications efficaces et de vérifier comment celles-ci sont perçues, dans le but de recommencer ou de réajuster le tir, au besoin.

Il faut aussi tenir compte des biais de sélection lorsque viendra le temps d'analyser les données. Par exemple, notre expérience sur le terrain montre qu'il faut un certain degré d'acceptation de sa condition pour visiter les groupes et les pages qui portent sur la NF1. Considérant que l'évolution et la gravité de la maladie sont impossibles à prévoir, la participation aux réseaux sociaux peut devenir une source d'angoisse, parce qu'il est possible d'y trouver des photos sur le profil ou les fils d'actualité de personnes dont les symptômes sont beaucoup plus apparents. Par contre, nous avons maintenu les autres stratégies de recrutement pour tenter de diminuer ce biais. Finalement, lors des recherches ciblant un groupe situé dans une région ou un pays spécifique, le recrutement par Facebook peut être imprécis. En ce qui nous concerne, nous ciblions seulement les Canadiens francophones ou anglophones. Or, nos participant(e)s d'intérêt se retrouvaient souvent dans les mêmes groupes ou pages Facebook que les Américains ou les Français, ce qui a fait en sorte que des adolescent(e)s d'autres pays ont tenté de participer. La plupart du temps, il était possible de les repérer à cause de leur adresse IP. Lorsque c'était le cas, pour ne pas créer de trop grande déception, nous leur avons fait part de notre projet d'étendre la recherche à d'autres pays. 


\section{Projet No. 2 : Points de vue de femmes enceintes sur les tests génomiques prénataux}

\section{Caractéristiques du projet de recherche}

Le deuxième projet réfère à une recherche qualitative menée dans la région de l'Estrie auprès de femmes enceintes âgées de 18 à 34 ans sans risque accru de mettre au monde un enfant atteint d'une maladie génétique. Dans la perspective de la médecine personnalisée, l'objectif général était de connaître les besoins et les points de vue de ces femmes, concernant les nouveaux tests prénataux et les informations génomiques qui leur semblaient souhaitables de connaître sur le fœtus. Réalisée dans le cadre d'une maîtrise 4 au moyen d'entrevues semi-dirigées, cette étude fait partie d'un programme de recherche sur la médecine personnalisée en périodes préconceptionnelle, prénatale et préimplantatoire.

\section{Contexte dans lequel les difficultés de recrutement ont été rencontrées}

Au départ, le recrutement s'annonçait facile à cause des particularités du terrain de recherche. D'une part, plus de 3000 enfants par année naissent en Estrie depuis les 20 dernières années (Institut de la statistique du Québec, 2017). D’autre part, nous bénéficions de la collaboration des infirmières de la Clinique de prélèvements en grossesse (CPG) du Centre hospitalier universitaire de Sherbrooke (CHUS), lesquelles étaient familières avec le recrutement en recherche. Nous pouvions aussi compter sur la participation des médecins d'une clinique médicale en région. Une fois que les femmes correspondant à nos critères d'inclusion étaient informées des grandes lignes du projet, celles qui étaient intéressées pouvaient contacter l'étudiante chargée de la réalisation du projet afin d'avoir davantage d'informations sur la problématique, ses objectifs et le déroulement de l'étude. Des affiches avaient aussi été posées à des endroits stratégiques, comme près des salles d'échographies du CHUS. Nous étions d'autant plus confiant(e)s du succès du recrutement que la recherche qualitative ne nécessite habituellement pas une grande quantité de participant(e)s pour atteindre la saturation des données ${ }^{5}$ (Anadon, 2006; Bowen, 2008; Fortin, 2010). Toutefois, après trois semaines, très peu de femmes enceintes s'étaient manifestées, et seulement deux participantes avaient pu être interviewées.

\section{Solution apportée}

Dans ces conditions, nous avons décidé de nous tourner une nouvelle fois vers le recrutement en ligne, en espérant rejoindre davantage de participantes potentielles. De par sa popularité, Facebook semblait une alternative prometteuse. Après avoir obtenu un amendement du CER du CIUSSS de l'Estrie - CHUS pour effectuer le recrutement via Facebook, nous avons identifié deux pages et un groupe Facebook estriens sur les thèmes de la grossesse. Nous les avons retenus tous les trois en raison de leur popularité et de leur niveau d'activité, soit 2000 mentions «J'aime» pour l'une des pages et 150 mentions «J'aime» pour l'autre et plus de 7500 membres pour le groupe 6 . L'étudiante a ensuite contacté les administrateurs/trices pour leur expliquer le projet de recherche et ils nous ont tous permis d'y publier notre invitation à participer l'étude. Publiée une fois par semaine

4. Projet de maîtrise de G. Lapointe, réalisé sous la direction de C. Bouffard et la co-direction de R. Drouin.

5. Critère scientifique servant à déterminer le moment où suffisamment de participant(e)s ont été recrutés lors d'une recherche qualitative. On parle de saturation théorique quand il y a redondance des données et quand aucune nouvelle information n'émerge des analyses.

6. Pour des raisons de confidentialité, les noms de la page et du Groupe ne sont pas divulgués. 
à partir du compte Facebook personnel de l'étudiante, cette invitation contenait des informations minimales sur le projet comme le sujet, les critères d'admissibilité, les coordonnées téléphoniques ou courrielles pour rejoindre l'étudiante ou la responsable du projet. Toutefois, la majorité des femmes intéressées sont entrées en contact via la messagerie du compte Facebook de l'étudiante. En l'espace de quatre semaines, nous avions réussi à recruter un nombre suffisant de femmes pour atteindre la saturation des données.

\section{Avantages perçus}

Dans le cadre de cette étude, le recrutement par Facebook s'est avéré très efficace en donnant accès à un grand nombre de participantes estriennes potentielles. En enlevant les intermédiaires à l'étape du recrutement (ex. : les infirmières, les médecins, etc.), Facebook a aussi favorisé un contact rapide et direct et contribué à réduire la distance entre les femmes enceintes et l'étudiante. En fait, il semble que le statut d'ami Facebook a créé un sentiment de familiarité qui a fait en sorte que les femmes se sont senties plus à l'aise de poser des questions sur le projet et que l'étudiante ait été en mesure de répondre plus rapidement et spontanément à leurs demandes d'informations. Facebook a aussi permis de conserver une distance appropriée entre l'équipe de recherche et les participantes potentielles en leur donnant le temps de réfléchir plus longuement aux questions qu'elles voulaient poser ainsi qu'à leur désir de participer à l'étude. Le fait d'être devant un écran d'ordinateur plutôt que devant un(e) professionnel(le) de santé ou de recherche offrait l'avantage de diminuer la pression ressentie par certaines femmes concernant leur décision d'accepter ou de refuser de participer à la recherche. De plus, le fait que les femmes enceintes prenaient l'initiative de poser des questions augmentait les probabilités qu'elles soient intéressées par le projet. Finalement, comme les participantes avaient la possibilité de voir la photo de profil de la personne qui allait réaliser les entrevues, ainsi qu'une partie du contenu du profil (ex : photos de profil, quelques publications, etc.), on peut se demander si l'utilisation d'un compte personnel a eu un effet rassurant en leur permettant de mettre un visage sur la personne qui les sollicitait et de s'en faire une idée.

\section{Inconvénients et questionnements}

Bien que cette méthode de recrutement ait été très efficace, elle a aussi comporté son lot de complications et des questionnements. Tout d'abord, sur le plan méthodologique, cette approche entraîne un biais de recrutement difficile à évaluer. Entre autres, certaines femmes enceintes n'ont pas de compte Facebook, n'utilisent pas leur compte régulièrement ou ne fréquentent pas le groupe ou les pages Facebook qui ont servi au recrutement. II pourrait aussi être difficile de rejoindre les personnes dont le niveau de littératie ne permet pas d'utiliser les réseaux sociaux. En vue de diminuer ces biais, nous avons maintenu les autres stratégies de recrutement plus usuelles. Enfin, même si l'accès au compte Facebook de l'étudiante a contribué à créer un climat de confiance et de familiarité, et même si le compte présente un certain niveau de confidentialité, on peut se demander si cette approche a pu diminuer la crédibilité de la chercheuse et le caractère professionnel de la recherche. Par exemple, le fait d'avoir accès à du contenu personnel de la personne qui allait mener les entrevues (photos ou publications) a pu emmener certains jugements ou a priori de la part des participantes potentielles. Également, puisque les projets de recherche se déroulent normalement dans des contextes institutionnels, par exemple, dans les universités ou les centres de recherche, le fait d'effectuer le recrutement via Facebook pouvait donner l'impression que la recherche était moins formelle. 


\section{Projet No. 3 : Préoccupations d'expert(e)s quant aux effets de l'intégra- tion chromosomique de l'herpès virus humain de type 6 sur la santé}

\section{Caractéristiques du projet de recherche}

Le troisième et dernier exemple porte sur une recherche qualitative exploratoire ayant pour objectif de connaître les préoccupations et les mises en garde de chercheurs/euses fondamentalistes et de médecins concernant les conséquences de l'intégration chromosomique de l'herpès virus humain de type 6 (ciHHV-6) sur la santé. L'herpèsvirus humain 6 (HHV-6) est le seul HHV capable d'intégrer les chromosomes des cellules (ciHHV-6). Il est transmis via les gamètes et les dons d'organes et de tissus. Ce phénomène touche plus de 1\% de la population mondiale et pourrait être associé à de nombreuses maladies (cancers, sclérose en plaques, etc.). Avec les nouvelles recherches et l'intérêt grandissant pour ce phénomène, les impacts connus du ciHHV-6 sur la santé pourraient se multiplier. Réalisée dans le cadre d'une maîtrise ${ }^{7}$, cette étude fait partie d'une recherche transdisciplinaire qui couvrait aussi les aspects cytogénétiques ${ }^{8}$ et génomiques du phénomène. C'est d'ailleurs en faisant une recension des écrits sur ces deux derniers aspects que nous avons constaté que certain(e)s chercheurs/euses fondamentalistes et médecins impliqué(e)s dans la recherche sur le ciHHV-6 ou sa prise en charge médicale étaient suffisamment préoccupé(e)s par la situation pour glisser des mises en garde cliniques, médicales et éthiques dans des articles purement scientifiques. Considérant qu'il est peu commun que des chercheurs/euses partagent de tels points de vue dans ce type de publications, le phénomène semblait suffisamment important pour qu'on s'y intéresse.

Nous avons donc élaboré un guide d'entrevue portant sur des thèmes tels : le diagnostic, la pathogénicité, les traitements, les impacts sur les greffes et les transplantations et l'intégration des connaissances sur le ciHHV-6 dans les services de santé. La découverte du ciHHV-6 étant relativement nouvelle (1993) (Luppi et coll., 2014), le phénomène est encore peu étudié. Le nombre d'expert(e)s est restreint et les participant(e)s potentiel(le)s dispersé(e)s partout dans le monde. C'est dans ce contexte que nous avons opté pour la même méthode de collecte de données qu'à la section 1.3, les entrevues écrites asynchrones en ligne, afin de pallier ce type de problèmes (Thoër, 2011).

\section{Contexte dans lequel les difficultés de recrutement ont été rencontrées}

À partir de ce moment, il fallait recruter des chercheurs/euses et des médecins qui étaient déjà conscientisé(e) s aux conséquences du ciHHV-6 sur la santé humaine. La première démarche pour rejoindre notre population cible a été de demander l'aide du comité scientifique de la Fondation HHV-6, comptant 22 membres correspondant à nos critères d'éligibilité. Malgré quelques premiers contacts encourageants, il n'y a pas eu de suite concrète à nos demandes.

Nous avons ainsi changé de stratégie de recrutement et avons décidé d'envoyer des invitations individuelles par courriel. De plus, considérant le petit nombre d'expert(e)s accessible via la Fondation HHV-6, pour être en mesure d'atteindre la saturation des données, nous avons ajouté à la liste les premier(ère)s auteur(e)s des articles répertoriés lors d'un scoping review que nous avons réalisée sur le ciHHV-6. Nous nous sommes limité(e)s aux premier(ère)s auteur(e)s, sachant que si nous n'étions pas en mesure d'atteindre la saturation des

7. Projet de maîtrise de V. Noël, réalisé sous la direction de C. Bouffard et la co-direction de R. Drouin et L. Flamand.

8. Objectif de la partie cytogénétique: Caractériser les sites d'intégration de l'ADN du ciHHV-6 dans le génome humain. 
données, l'ensemble des auteur(e)s pourrait être contactés par la suite. De plus, les participant(e)s potentiel(le) s étaient invité(e)s à transmettre l'invitation à leurs collègues.

Considérant que, dans cet univers professionnel, un courriel provenant d'un médecin-chercheur avait plus de chance d'être lu que celui d'une étudiante, les invitations ont été envoyées par le Dr Drouin, le médecin généticien et chercheur responsable de la partie génétique du projet. Deux rappels ont dû être envoyés pour ce type de recrutement.

Par contre, un des principaux problèmes a été de trouver les adresses courriel des expert(e)s identifié(e) s. D’abord, celles des membres de la Fondation HHV-6 n'étaient pas disponibles sur le site. Nous les avons cherchés en référant à leurs institutions d'appartenance ou à leurs articles. Toutefois, comme on pouvait s'y attendre, les courriels de correspondance n'étaient pas toujours ceux des premier(ère)s auteur(e)s. Malgré des recherches approfondies sur Internet, cette méthode n'a pas permis de trouver toutes les adresses courriel. De plus, certaines adresses ont provoqué des messages d'erreurs automatiques indiquant qu'elles n'étaient plus valides, ou encore nos courriels d'invitation étaient considérés comme des messages suspects et placés dans les courriers indésirables des destinataires. Au final, ce mode de recrutement n'a pas permis de certifier que tous les participant(e)s potentiel(le)s avaient été rejoint(e)s. Pour atteindre nos objectifs, il fallait une autre façon de procéder.

\section{Solution apportée}

Dans ces conditions, nous avons soumis une demande d'amendement au CER du CRCHUS, afin de recruter les participant(e)s en recourant aux réseaux sociaux. Facebook a été écarté puisque ce réseau social est trop lié à la vie personnelle des gens pour une recherche où on veut recruter des participant(e)s en se basant sur leur parcours professionnel. Les réseaux sociaux ResearchGate et LinkedIn, reconnus comme des réseaux professionnels, correspondaient mieux à nos objectifs de recrutement. À partir de ces réseaux nous avons pu contacter des participant(e)s potentiel(le)s additionnel(le)s pour qui les invitations individuelles par courriel n'avaient pas donné suite. Cette méthode de recrutement nous a aussi offert la possibilité de valider l'identité des participant(e)s en nous basant sur la description de leurs parcours, de leurs publications et de leurs intérêts de recherche. Ce qui s'est avéré moins fastidieux que de faire des vérifications en cherchant chacune de ces informations sur le web. Ces expert(e)s étaient ensuite contacté(e)s via les boîtes de messagerie de LinkedIn ou de ResearchGate. C'est finalement dans un cadre professionnel qu'ils/elles ont reçu notre message les invitant à participer à notre projet de recherche. Ce message était accompagné d'informations concernant notre équipe de recherche, la problématique de l'étude et ses objectifs. Il contenait aussi le lien hypertexte menant au site d'hébergement du formulaire d'information et de consentement, ainsi qu'à l'entrevue écrite asynchrone en ligne. 
À partir des boîtes de messagerie liées à leur plateforme respective, ResearchGate et Linkedln permettent d'éviter les contacts intermédiaires (collègues, établissement d'attache, etc.) et d'établir un contact direct et rapide avec les personnes recherchées. Ce, tout en conservant la formule courriel, une approche idéale pour communiquer avec des médecins et des chercheurs/euses dispersé(e)s à travers différents fuseaux horaires et dont l'emploi du temps est très chargé. De plus, les communications en format texte représentent un réel avantage lorsque la langue première des personnes sollicitées n'est ni l'anglais ni le français. Les barrières linguistiques deviennent beaucoup plus évidentes que lors d'une entrevue téléphonique, par exemple. Enfin, comme ResearchGate et Linkedln sont des réseaux sociaux qui permettent de suivre des professionnel(le)s et des scientifiques, ils offraient le cadre professionnel que nous voulions conserver pour notre recrutement.

\section{Inconvénients perçus}

Si ResearchGate et Linkedln permettent d'échanger à partir d'une boîte de messagerie, ils ont le désavantage de ne pas donner accès à l'adresse courriel. II n'a donc pas été possible de conserver le même mode de recrutement pour l'ensemble des expert(e)s, certain(e)s ayant été contacté(e)s par courriel, d'autres par les réseaux sociaux professionnels. Malgré les avantages et la popularité de Linkedln et de ResearchGate en Amérique du Nord, ailleurs dans le monde, ces réseaux sont moins utilisés. C'est d'ailleurs le cas en Europe et en Asie, où on semble préférer des réseaux sociaux professionnels comme Academia et Viadeo.

Par ailleurs, il n'est pas possible de valider l'identité des candidat(e)s dont les informations sont rédigées dans une autre langue. Cet inconvénient ajoutait également à la complexité de valider l'identité lorsque plusieurs comptes avaient des descriptifs similaires tels que le même prénom, le même nom de famille ou la même région de résidence. Dans ces rares cas, les personnes n'ont pas été contactées parce que comme nous ne pouvions les identifier avec certitude, il était impossible de savoir si elles répondaient aux critères d'inclusion.

\section{Dimensions éthiques de l'utilisation des réseaux sociaux pour le recrute- ment à la recherche}

Il y a déjà plusieurs années que les chercheurs/euses utilisent les réseaux sociaux pour recruter des candidat(e) s susceptibles de participer à leurs projets de recherche. Un des grands avantages de ce mode de recrutement est de donner, à très peu de frais, accès à un grand nombre de participant(e)s potentiel(le)s (Frippiat et Marquis, 2010). Il offre aussi une panoplie d'outils et d'applications web pour communiquer ou échanger avec les candidat(e)s sur des plages d'espace et de temps variables, en direct ou asynchrones (Jean, 2015). Le recrutement en ligne s'avère particulièrement efficace, notamment pour la recherche qualitative en santé (Frandsen et coll., 2013; Chu et Snider, 2013).

Dans ces conditions, on peut se demander pourquoi nous en sommes encore à nous interroger sur les meilleures mesures à prendre pour que ce mode de recrutement respecte les normes de l'éthique de la recherche. D'ailleurs, plusieurs expert(e)s s'entendent pour dire que le recrutement en ligne ne pose pas de problèmes éthiques fondamentalement différents que ceux induits par les méthodes conventionnelles (Ess, 2002; Whitehead, 2007; Buchanan et Hviddak, 2009). Pour ces auteur(e)s, que le recrutement se fasse sur le web, par téléphone ou en face à face, les enjeux éthiques demeurent les mêmes, soient, entre autres : protéger la personne 
et son intégrité, ne pas causer plus de tort que de bien, s'assurer de la justice et de l'équité de nos pratiques et se responsabiliser quant à leurs conséquences. À cette liste, nous ajouterons l'intégrité scientifique.

Dans cette perspective, trois phénomènes peuvent expliquer en partie pourquoi nous persistons à nous interroger sur l'encadrement éthique du recrutement en ligne. D’une part, «[...] Internet a facilité considérablement "l'accès au terrain", c'est-à-dire à des corpus de données, voire à des sujets humains susceptibles de participer à des enquêtes, si bien que l'on a vu proliférer une forme de "fast research"», souvent le fait de chercheur(euse) s insuffisamment préparé(e)s, ou en formation et n'étant pas toujours respectueux/euses des droits et de la dignité des participant(e)s [...]»(Latzko-Toth et Proulx, $2013: 32$ ).

D’autre part, «[...] il existe encore un débat considérable concernant l'éthique de la recherche sur Internet, notamment parce qu'Internet est encore dans une phase de développement et que de nouveaux phénomènes continuent d'apparaître» ${ }^{9}$ (Fielding, Lee et Blank, 2008 : 21). Dans ces conditions, les expert(e)s de l'éthique et les membres des comités d'éthique de la recherche (CER) ne sont pas toujours en mesure d'appuyer leurs réflexions et leurs analyses sur une connaissance approfondie et pragmatique des caractéristiques du web et des réseaux sociaux (Teitcher et coll., 2015). Ce qui fait en sorte que les craintes à la base des mesures éthiques pour le recrutement en ligne sont le plus souvent prospectives. «On reconnaît toutefois de plus en plus que les enjeux éthiques ne peuvent être pleinement prédits et planifiés, et que la recherche éthique sur les interactions humains-ordinateurs peuvent nécessiter une approche plus subtile et souple que les stratégies préconisées par les comités d'éthique ${ }^{10}$ (Fielding, Lee et Blank, 2008 : 21). De plus, ce qui apparaît éthique ou non est souvent modulé par les cadres de références éthiques personnels, culturels et professionnels des chercheurs (Waycott et coll., 2016). Par conséquent, les exigences éthiques ne sont pas toujours adaptées aux réalités du web et les mesures encadrant les projets de recherche sont souvent établies au cas par cas selon différentes positions des chercheurs/euses et des CER (Bouffard, 2017). Ce contexte ne permet pas encore de favoriser l'élaboration de solutions proactives et durables. C'est-à-dire, avec la capacité de s'adapter aux changements sociotechnologiques et à l'évolution des besoins en matière de recrutement, tout en apportant des réponses acceptables du point de vue éthique et applicables aux situations similaires, qui perdureront dans le temps.

Enfin, ce que l'utilisation du web vient changer pour plusieurs auteurs, c'est «[...] la nature des risques encourus et la capacité du chercheur à évaluer ces derniers» (Jean, 2015 : 14). Par exemple, dans le cadre de la recherche en biomédecine, alors que les risques d'atteinte à l'intégrité des personnes dans les approches de recherches étaient plutôt physiologiques, c'est pour la vie privée que la recherche en ligne peut représenter une menace (Kraut et coll., 2004). Si la recherche en sciences sociales risque rarement de porter atteinte à l'intégrité physique, les problèmes de confidentialité et de vie privée avec lesquels elle a l'habitude de composer sont décuplés sur le web.

Dans cette perspective, ce ne sont pas les problèmes éthiques, mais plutôt les défis éthiques qui sont différents lorsque le recrutement se fait en ligne (Mann, 2003). Sur la base de la littérature et de l'expérience que nous en avons, un des premiers défis à relever sera d'opérer un changement dans les mentalités, qui devra commencer par considérer que les dimensions éthiques du recrutement en ligne demeurent intrinsèquement liées aux utilisateurs/rices, aux conditions d'utilisation de l'outil et aux caractéristiques des plateformes et des

9. Traduction libre: « [...]there is still considerable debate about the ethics of Internet research - not least because the Internet is still in a formative phase and new phenomena continue to emerge. In this chapter, we will discuss some of the major issues that have been debated and give some indication of how to go about addressing them. » (Fielding, Lee et Blank, 2008 : 21)

10. Traduction libre: «There is, however, growing recognition that ethical issues cannot be fully predicted and planned for, and that conducting ethical $\mathrm{HCl}$ research may require a more subtle and flexible approach than the strategies advocated by ethics review boards ». (Fielding, Lee et Blank, $2008: 21$ ) 
réseaux sociaux exploités (Bouffard, 2017). Par conséquent, les mesures réglementaires visant à encadrer le recrutement en ligne devront tenir compte du fait que les réseaux sociaux n'atteignent pas les mêmes catégories de personnes, n'ont pas tous les mêmes fonctionnalités, ne permettent pas de faire les mêmes types de sollicitation ou d'interactions et ont des niveaux de confidentialité qui diffèrent. Ce qui exigera la contribution d'expert(e)s de la programmation et une connaissance approfondie des particularités des réseaux sociaux. II faudra aussi garder en tête que la validité et l'applicabilité d'une norme ou d'une ligne directrice éthique pour la recherche en ligne sera toujours tributaire des connaissances qu'en auront les personnes, les expert(e)s ou les décideur(euse)s qui les élaboreront.

Dans ce contexte, les trois recherches dont il a été question dans ce chapitre nous ont amenées à réfléchir sur les défis à relever par rapport à l'équité dans la représentativité et dans l'accès au bénéfice de recherche, à la confidentialité et à la protection de la vie privée, au respect de la personne et de l'intégrité de ses identités, ainsi qu'à l'importance d'une approche collaborative et co-constructive avec les CER.

\section{Équité, représentativité et accès au bénéfice de recherche}

L'idée d'utiliser les réseaux sociaux est venue d'un besoin de trouver une alternative aux difficultés de recrutement. Toutefois, le recrutement en ligne exige beaucoup de souplesse et une certaine expertise des technologies web pour être en mesure de s'adapter aux différents contextes de recherche. Comme nous l'avons vu, nos premières expériences ont nécessité certains ajustements. Aujourd'hui, lorsque le web ou les réseaux sociaux correspondent aux contextes et aux phénomènes que nous voulons étudier et qu'ils permettent de rejoindre les populations recherchées, nous priorisons d'emblée le recrutement et même la recherche en ligne.

Par contre, comme nous l'avons fait pour les trois études présentées dans ce chapitre, afin d'éviter certains biais (supra), nous ne nous restreignons pas au recrutement en ligne

Au niveau éthique, la diversification des modes de recrutement permet de réduire les risques que certaines personnes ou communautés soient exclues ou sous-représentées. Pour le recrutement en ligne, il peut s'agir de personnes peu familières avec Internet, qui n'y ont pas accès, ou qui ont un trop faible niveau de littératie. La mise à l'écart ou un accès limité à une partie de la population d'intérêt pourrait donner une image tronquée du phénomène à l'étude en ne tenant pas compte des problèmes, des préoccupations et des positions de ces personnes. Ce qui aurait pour conséquence de les marginaliser encore plus, en les empêchant de bénéficier des mesures ou des solutions issues des résultats d'une étude qui aurait pu leur être utile, ou de tirer un plus grand bénéfice de leur participation à la recherche (Bouffard et coll., 2017). La flexibilité dans les modes de recrutement tend ainsi à répondre au principe de justice (EPTC 2, 2014), principalement pour ce qui concerne l'équité dans la représentativité et dans l'accès au bénéfice de la recherche, de même qu'en évitant, dans certains cas, de contribuer à renforcer les divisions sociales déjà en place (Mann, 2003).

Au niveau scientifique, l'utilisation exclusive du recrutement en ligne risque ainsi d'avoir un impact sur la crédibilité des résultats. La diversité des participant(e)s est essentielle pour les devis qualitatifs exploratoires, dont la fonction est de générer des données qui permettront d’obtenir le portrait le plus complet possible d'un phénomène nouveau ou peu étudié (Corbière et Larivière, 2014). Dépendamment des objectifs de recherche, l'absence de certain(e)s acteurs/trices clés peut affecter la qualité des données en ne permettant pas de couvrir l'ensemble des enjeux propres à une problématique. La validité et la transférabilité des résultats obtenus seraient aussi mises en doute, ces derniers se limitant aux individus recrutés. Comme le mentionne Pittenger (2003), les chercheurs/euses ont la responsabilité éthique de veiller à ce que la recherche qu'ils effectuent soit 
de haute qualité et que les conclusions tirées soient déduites à partir des données recueillies (Pittenberg, 2003). La qualité éthique d’un projet de recherche est étroitement liée à la qualité scientifique de ses retombées.

Pour la recherche que nous avons menée auprès des femmes enceintes, par exemple, il était essentiel d'inclure le plus de catégories de participantes possible si nous voulions réellement connaître le type d'informations qu'elles souhaiteraient avoir sur le génome du fœetus qu'elles portent. Cette condition est encore plus importante lorsque nos recherches visent à produire des données qui pourraient guider les médecins, les patient(e) s et les décideurs politiques dans leurs prises de décision concernant les pratiques, les tests, les services et les politiques de santé à prioriser.

\section{Confidentialité et protection de la vie privée}

Les risques d'atteinte à la vie privée sont beaucoup plus élevés en ligne qu'en personne, par téléphone ou par la poste (Bouffard, 2017). Les réseaux sociaux comme Facebook, par exemple, regorgent d'informations personnelles et de photos accessibles lorsque le profil des participant(e)s n'est pas en mode privé. Par conséquent, il faut donner toute son importance au «[...] caractère perçu de la privacité ou de la publicité d'un espace de communication, les internautes ayant tendance à considérer comme "intime" un média qui facilite l'expression d'émotions» (Barnes, 2004 : 206-207). D’un point de vue éthique, les chercheurs/euses doivent demeurer vigilants, car il sera toujours difficile de distinguer ce qui appartient à la sphère du public et ce qui appartient à la sphère du privé, «[...] une distinction mise à mal par les nouvelles formes d’interaction médiatisée par ordinateur et leur mise en visibilité par le web» (Latzko-Toth et Proulx, 2013:32; Pastinelli, 2011). Nous avons d'ailleurs eu à composer avec ce type de problème lors des projets de recherche sur les perceptions des adolescent(e)s atteint(e)s sur la NF1 et sur les points de vue des femmes enceintes concernant les tests génomiques prénataux.

Pour la recherche réalisée en collaboration avec les adolescent(e)s atteints de NF1, l'assistante de recherche responsable du projet avait créé un compte à l'usage exclusif du projet de recherche. En ayant la possibilité de lire les propos et les échanges tenus sur les groupes Facebook en lien avec la NF1 dont elle était membre, ainsi que de ses amis Facebook, elle avait accès à des informations personnelles qu'elle n'aurait pas obtenues avec des techniques conventionnelles. Les problèmes éthiques sont de deux ordres ici. D'une part, lorsque les utilisateurs/trices échangeaient sur des sujets intimes, ils/elles n’étaient pas nécessairement conscient(e) s qu'une assistante de recherche était membre du groupe. Même si c'était clairement indiqué sur notre page, rien ne garantissait qu'ils l'avaient consultée. Ce problème est d'autant plus important que les communautés virtuelles ne s'attendent pas à ce que leurs réseaux fassent l'objet d'une recherche (Chen et coll., 2004; Eysenbach et Till, 2001; McKee et Porter, 2009; Sveningson, 2004; Zimmer, 2010). D'autre part, que peut-on faire avec les informations obtenues par défaut? Peuvent-elles être utilisées indirectement afin d'améliorer les connaissances sur le contexte ou s'agit-il d'informations confidentielles? Dans quelle mesure l'information sur Internet peut être considérée comme étant intentionnellement publique (Odwazny, 2017)?

Dans le cadre de l'étude menée auprès des femmes enceintes, l'étudiante avait décidé d'utiliser son compte Facebook personnel pour favoriser une approche personnalisée. Toutefois, après les entrevues, deux participantes désirant perpétuer la relation en dehors du cadre de la recherche lui ont fait parvenir des demandes d'amitié Facebook, ce qui impliquait qu'elles auraient aussi accès aux informations du profil de l'étudiante. Si ces demandes témoignent de la force du lien de confiance établi pendant l'entrevue, nous ne pouvions que nous interroger sur le bien-fondé scientifique et éthique d'une telle proximité pour ce type d'étude. 
Pour les travaux sur le ciHHV-6, les profils des professionnel(le)s et des chercheurs/euses étaient publics et accessibles à tous les membres des réseaux Linkedln et ResearchGate. Il était même possible de leur écrire directement en passant par le réseau. Dans la mesure où le message d'invitation était professionnel et que les participant(e)s potentiel(le)s n'avaient qu'à cliquer sur l'hyperlien menant à l'entrevue, leur anonymat et leur vie privée n'étaient pas compromis. Le problème était de conserver l'anonymat une fois les résultats publiés (EPTC2, 2014). En raison de leur nombre limité, il aurait pu être possible d'identifier certain(e)s participant(e)s, en croisant les propos ou les données sociodémographiques avec les informations disponibles sur Linkedln, sur ResearchGate, lors d'évènements scientifiques et même à partir des informations sur le web où dans les bases de données comme Google Scholar et Pubmed.

Peu importe le type de recherche, des mesures minimales ont pu être prises pour éviter les problèmes dont il vient d'être question ou pour en limiter les conséquences. D’abord, afin de ne pas porter atteinte à la vie privée des participant(e)s à nos recherches, nous évitions de consulter leurs profils Facebook même s'ils n'étaient pas tous en mode privé. Par contre, en devenant membre de groupes tels que les groupes Facebook et en acceptant des demandes d'amitiés, il devenait impossible de faire complètement abstraction des informations accessibles sur les réseaux sociaux à partir desquels nous recrutions. Dans ces conditions, les informations accessibles sur les réseaux sociaux, et surtout les informations personnelles, doivent être considérées pour ce qu'elles sont, c'est-à-dire, des fragments de réalité virtuelle qui doivent être mis en contexte en gardant en tête que «[...] les formes identitaires projetées sur le web s'adressent à des publics différents et ont des visées multiples» (Cardon, 2011 : 142). Pour respecter cette réalité, autant pour des raisons éthiques que par rigueur scientifique, aucune des informations auxquelles nous avons eu accès sur les pages et les groupes Facebook n’a été volontairement utilisée à des fins de recherche ou dans les publications. Et ce, tout en ayant conscience qu'elles auraient nécessairement eu un impact plus ou moins grand sur nos analyses.

Ensuite, considérant que les études dont il est question ici exigent de développer une relation de proximité et un haut niveau de confiance, certaines questions éthiques se posent non seulement en regard de la protection de la vie privée des participant(e)s, mais aussi de celle des chercheurs/euses. L'utilisation d'un compte personnel pour le recrutement risque de placer chercheurs/euses et participant(e)s dans des situations délicates, comme ce fut le cas lorsque l'étudiante a reçu les demandes d'amitiés. Accepter pouvait permettre une intrusion dans la vie privée de l'étudiante qui outrepassait les limites de la recherche. Refuser pouvait leur laisser croire que la relation n'avait été qu'utilitaire. Considérant la nature du projet, le petit nombre de personnes ayant fait cette demande et les informations délicates et intimes que les participantes avaient livrées, par souci de réciprocité et pour ne pas altérer leur confiance, il a été décidé d'accepter leur demande d'amitié. Si recruter via les réseaux sociaux implique d'être prêts à rendre certaines informations personnelles accessibles, cette ouverture se limite aux besoins de la recherche. D’autant plus que la création d'un compte spécifique au projet permet d'informer les personnes qui ont fait des demandes d'amitiés sur les avancées et les résultats de l'étude, qu'ils/ elles y aient participé ou non.

Certaines fonctions Facebook permettent aussi de masquer le suivi des activités des profils des participant(e) s. On limite ainsi les informations qui nous parviennent en évitant qu'elles s'affichent en continu sur notre fil d'actualité. II est aussi possible de modifier nos paramètres de confidentialité pour faire en sorte que les participant(e)s aient un accès limité à nos informations personnelles et à nos activités.

Enfin, tel que recommandé par l'Énoncé de politique des trois Conseils, en raison des spécificités ou du petit nombre de personnes ou de professionnel(le)s concerné(e)s par nos recherches, il était nécessaire de «[...] prendre des mesures raisonnables pour prévenir l'identification par mégarde de personnes ou de groupes 
dans des publications ou lorsqu'ils utilisent d'autres moyens de diffusion» (EPTC2, 2014: 68). Même si la cueillette des données des trois études qui ont fait l'objet de ce chapitre garantissait l'anonymat, certains extraits d'entrevue, des données identificatoires, ou certains résultats pouvaient permettre de reconnaître la personne, le lieu ou l'institution dont il était question. Sans compter que, le regroupement de fragments publiés sur le web, il est facile de retracer un individu, ainsi que les endroits où ils ont été partagés. Afin d'éviter d'isoler les éléments qui pourraient permettre d'identifier les participant(e)s à la source de certaines informations, nous avons pris soin de changer l'angle d'approche ou de regrouper certains thèmes et sous-thèmes dans la présentation des résultats.

\section{Respect de la personne et de son intégrité}

Pour Ess (2006), ce qui est véritablement nouveau avec la recherche en ligne, c'est l'apparition de nouvelles formes d'identité et de communautés (Ess, 2006).

En effet, la réussite des plateformes relationnelles du web 2.0 doit beaucoup au fait que les personnes y exposent différents traits de leur identité. Ce phénomène renvoie à deux dynamiques des processus d'individualisation observables dans les sociétés contemporaines : un processus de subjectivation, qui conduit les personnes à extérioriser leur identité dans des signes qui témoignent moins d'un statut incorporé et acquis que d'une capacité à faire (écrire, photographier, créer...); et un processus de simulation, qui les conduit à endosser une diversité de rôles exprimant des facettes multiples, et plus ou moins réalistes, de leur personnalité (Cardon, 2011 : 142).

Même dans le contexte de réseaux sociaux professionnels, la vigilance est de mise. Les informations qu'on y trouve, comme le rapporte Cardon pour l'ensemble des réseaux sociaux, sont «[...] loin d'être des données objectives, attestées, vérifiables et calculables, le patchwork désordonné et proliférant de signes identitaires produits sur les réseaux sociaux est tissé de jeux, de parodies, de pastiches, d'allusions et d'exagérations» (Cardon, $2011:$ 142).

Dans de telles conditions, les précautions éthiques que les chercheurs/euses doivent prendre pour protéger les identités en ligne ne sont pas nécessairement les mêmes que celles élaborées pour le modèle traditionnel des sujets humains (Ess, 2006). Il peut aussi s'avérer difficile de vérifier l'identité des participant(e)s et de leur porter l'assistance en cas de besoin (British Psychological Society, 2007). Avec l'usage des pseudonymes et des identités multiples, quoi que ce soit possible, il est beaucoup plus compliqué de connaître l'identité réelle de la personne avec laquelle on échange (British Psychological Society, 2007; Côté, 2012).

Également, la mise à distance induite par les réseaux sociaux et la création d'identités différentes «[...] peut dissoudre notre sens des responsabilités» (Chardel et Reber, 2011 : 152). D’ailleurs, ces auteurs rappellent la mise en garde de Lévinas «[...] contre ce risque latent de la perte des visages ou d'une proximité qui est nécessaire au développement d'une certaine "conscience d'autrui"» (Chardel et Reber, 2011:153; Levinas, 1961). Cette réflexion nous place devant un paradoxe éthique dont les conséquences ne sont pas négligeables. D’une part, le respect de la personne, de son intégrité et de sa vie privée impose de ne pas aller consulter les profils ou les informations personnelles que les participant(e)s publient sur les réseaux sociaux. D’autre part, ne pas consulter les profils vient exacerber l'effet de distance que peuvent provoquer les interactions en ligne, alors que le contraire donne réalité à la personne devant nous, peu importent ses stratégies identitaires. 


\section{Travail collaboratif avec le comité d'éthique}

Enfin, pour relever les défis éthiques du recrutement en ligne, il est essentiel que les chercheurs/euses et les membres des CER travaillent ensemble. Pourtant, de nombreux CER ne sont pas encore familiers avec la recherche sur Internet et en savent peu sur les meilleures mesures à mettre en place pour protéger tous les acteurs/trices concerné(e)s (Mckee et Porter, 2009). Pour Mckee et Porter (2009), l'éthique devrait être considérée comme un processus continu de réflexion, d'analyse et d'action et, comme chaque communauté est unique, ils recommandent l'utilisation d'approches heuristiques plutôt que l'adoption de règles éthiques rigoureuses. Ainsi, contrairement à Jean (2015) et Kraut et coll. (2004) qui soutiennent que le web vient changer la nature des risques encourus, Odwazny (2014) soutient que, comme les principes et les règlements éthiques pertinents sont les mêmes pour la recherche conventionnelle et pour la recherche en ligne, cette dernière ne pause pas de problèmes éthiques de nature différente. Ce serait plutôt le degré d'intensité des risques qui serait différent.

Pour ce qui nous concerne, c'est cette façon de procéder et de percevoir les risques inhérents à la recherche en ligne qui a été approuvée par notre CER. À chaque étape, au fil des problèmes qui ont émergé et surtout des propositions qu'on leur faisait, les membres de nos CER ont fait preuve d'une ouverture exceptionnelle. Nos échanges ont permis des avancées réflexives et constructives qui ont mené à l'élaboration de protocoles adaptés au type de recherche que nous faisions, mais surtout au type de participant(e)s avec qui nous interagissions sur le web.

\section{Conclusion}

Dans ce chapitre nous avons présenté les difficultés que nous avons rencontrées en recourant à trois différents types de recrutement à partir des réseaux sociaux. Ces expériences, ainsi que le cheminement réalisé en collaboration avec les membres de notre CER, nous ont permis d'identifier certaines conditions préalables pour être en mesure de nous conformer aux exigences de l'éthique de la recherche et de dégager certains défis éthiques à relever.

Au niveau des conditions préalables pour les équipes de recherche:

1. disposer des moyens logistiques et financiers, ainsi que de l'expertise nécessaire, pour assurer une interface fonctionnelle et des mesures de sécurité aptes à protéger l'anonymat et la vie privée des participant(e)s et des chercheurs/euses (Bouffard et coll., 2017);

2. avoir un certain niveau de connaissances de la population à recruter et de ses habitudes sur le web, pour identifier les réseaux, groupes, pages et sites susceptibles de nous donner accès aux catégories de participant(e)s pertinentes;

3. consulter les admisistrateurs/trices, afin de connaître le fonctionnement et la culture des groupes auxquels on s'adresse;

4. tenir compte de leurs réserves et de leurs recommandations concernant les modes de recrutement et de collecte de données qu'on envisage de réaliser permettra d'éviter plusieurs écueils éthiques (Chen et coll., 2004); et,

5. assurer la correspondance et la flexibilité de l'approche méthodologique choisie par rapport aux objectifs de recherche et, au besoin, à un changement de stratégie de recrutement. 
Cinq défis éthiques ont particulièrement retenu notre attention :

1. Disposer d'outils informatiques soignés et adaptés à la population, à la problématique, au contexte et aux objectifs de la recherche (Couper et coll., 2007). Les problèmes que nous avons rencontrés dans le cadre de la recherche avec les femmes enceintes et avec le recrutement des adolescent(e)s et des expert(e)s du ciHHV-6 nous rappellent à quel point il est important d'utiliser les bons outils et les bons réseaux pour rejoindre les participant(e)s escompté(e)s;

2. Établir un rapport de confiance avec les participant(e)s dès le début, parce qu'un bon rapport fait partie des stratégies à adopter pour tenter de remédier aux problèmes éthiques qui peuvent survenir dans le contexte de la recherche en ligne (Ess, 2006; Mann et Stewart, 2000);

3. Anticiper et évaluer les risques de préjudices potentiels pour les participant(e)s et les chercheurs/euses avant de commencer l'étude, ainsi qu'au moment de la rédaction et de la diffusion des résultats;

4. Établir la marche à suivre pour tenter de distinguer ce qui relève de la vie publique et de la vie privée. Pour Zimmer (2010), le recueil et la conservation d'informations personnelles sur les médias sociaux dans le but de les rendre accessibles pour des recherches ultérieures représente :

[...] un affront à la dignité humaine et à la capacité des internautes à contrôler la circulation de leurs informations personnelles. II [Zimmer] soutient qu'on ne peut comparer l'information publiquement disponible sur les réseaux sociaux à l'information disponible par observation sur la place publique puisqu'une grande quantité de renseignements personnels peut être extraite du web, des renseignements plus ardus à obtenir par l'observation dans le monde réel (Côté, 2012: 36).

L'ÉPTC 2 rappelle aussi que «[la] vie privée est respectée si la personne a la possibilité d'exercer un certain contrôle sur l'usage de ses renseignements personnels en donnant ou en refusant son consentement à la collecte, à l'utilisation ou à la divulgation d'informations à son sujet [...]» (ÉPTC 2, 2014 : 62).

5. Respecter la personne et son intégrité, en tenant compte de ses stratégies identitaires:

[Sur le web] le/la chercheur/euse est invité(e) à dépasser les conditions d'utilisation des sites et la protection conférée par l'usage des pseudonymes. Il se préoccupera du contexte, c'est-à-dire de la perspective des internautes quant à la sensibilité du sujet dans le cadre de son protocole de recherche, de la présence de renseignements identificatoires et de leurs vulnérabilités (Côté, 2012 : 39).

Nous sommes parfaitement conscient(e)s que ces défis sont loin de former une liste exhaustive. Par contre, lorsque les précautions adéquates sont prises, les recherches en ligne ne comportent pas plus de risques pour les humains que les recherches comparables faisant appel à d'autres formes de recrutement et de collecte de données. Chercheurs/euses, participant(e)s, membres des CER et sociétés, c'est ensemble qu'il faut trouver un équilibre entre l'importance des dommages potentiels aux participant(e)s et les avantages de la recherche pour l'individu et la société en général (Fielding et coll., 2008). Et ce, en passant par la mise en commun de nos expériences sur le terrain et non seulement par une attitude prospective basée sur nos craintes.

Enfin, considérant la mouvance du développement web, pour faire face aux enjeux éthiques soulevés par le recrutement en ligne, il est essentiel de ne pas attendre qu'une éthique institutionnalisée vienne normaliser nos pratiques. Rien ne nous empêche d'adopter une posture éthique basée sur la réciprocité. S'abstenir de lire les informations personnelles ou de regarder les photos et publications des participant(e)s, même s'ils sont facilement accessibles, gérer les paramètres de confidentialité pour en minimiser l'accès et ne pas utiliser pour la recherche les renseignements qu'il est impossible d'ignorer en sont quelques exemples. II semble aussi essentiel d'accepter les demandes d'amitié par esprit de réciprocité et d'équité. II faut aussi envisager d'adapter nos styles de rédaction et, parfois même, de ne pas divulguer certains résultats s'ils risquent d'entraîner la 
réidentification de participant(e)s. Une posture éthique délibérée, en phase avec une règle peu originale, mais fondamentale, qui implique de traiter les autres comme nous voudrions être traité(e)s.

\begin{tabular}{|c|c|c|c|c|}
\hline \multirow[t]{2}{*}{ Tableau $1:$} & \multicolumn{4}{|c|}{ Caractéristiques des projets de recherche } \\
\hline & POPULATION CIBLE & $\begin{array}{l}\text { COLLECTE DES } \\
\text { DONNÉES }\end{array}$ & RECRUTEMENT INITIAL & $\begin{array}{l}\text { RECRUTEMENT EN } \\
\text { LIGNE }\end{array}$ \\
\hline \multicolumn{5}{|l|}{ PROJETS } \\
\hline $\begin{array}{l}\text { Projet no. } \mathbf{1} \text { : } \\
\text { Perceptions d'adoles- } \\
\text { cent(e)s canadien(ne)s } \\
\text { atteint(e)s de NF1 }\end{array}$ & $\begin{array}{l}\text { Adolescent(e)s cana- } \\
\text { dien(ne)s (15 à } 19 \text { ans) } \\
\text { atteint(e)s de NF1 }\end{array}$ & $\begin{array}{l}\text { Entrevues écrites } \\
\text { asynchrones en ligne }\end{array}$ & $\begin{array}{l}\text { Médecins collaborateurs } \\
\text { Associations }\end{array}$ & $\begin{array}{l}\text { Groupes FB } \\
\text { Pages FB } \\
\text { Publications } \\
\text { commanditées }\end{array}$ \\
\hline $\begin{array}{l}\text { Projet no. } \mathbf{2} \text { : } \\
\text { Besoins et points de vue } \\
\text { de femmes enceintes } \\
\text { estriennes, concernant les } \\
\text { nouveaux tests géno- } \\
\text { miques prénataux }\end{array}$ & $\begin{array}{l}\text { Femmes enceintes } \\
\text { (18 à } 34 \text { ans), sans risque } \\
\text { accru d'avoir un enfant } \\
\text { avec maladie génétique }\end{array}$ & Entrevues semi-dirigées & $\begin{array}{l}\text { Infirmières (Clinique de } \\
\text { prélèvement) } \\
\text { Médecins d'une clinique } \\
\text { estrienne } \\
\text { Affiches }\end{array}$ & $\begin{array}{l}\text { Groupes Facebook } \\
\text { Pages Facebook }\end{array}$ \\
\hline $\begin{array}{l}\text { Projet no. } 3 \text { : } \\
\text { Préoccupations de cher- } \\
\text { cheurs/euses et méde- } \\
\text { cins, quant aux effets du } \\
\text { ciHHV-6 sur la santé }\end{array}$ & Expert(e)s du ciHHV-6 & $\begin{array}{l}\text { Entrevues écrites } \\
\text { asynchrones en ligne }\end{array}$ & $\begin{array}{l}\text { Fondation HHV-6 } \\
\text { Auteurs d'articles } \\
\text { scientifiques }\end{array}$ & $\begin{array}{l}\text { Linkedln } \\
\text { ResearchGate }\end{array}$ \\
\hline
\end{tabular}




\begin{tabular}{|c|c|c|}
\hline Tableau 2 & \multicolumn{2}{|c|}{ Avantages et inconvénients de Facebook, LinkedIn et ResearchGate } \\
\hline & AVANTAGES & INCONVÉNIENTS \\
\hline \multicolumn{3}{|l|}{ PLATEFORME } \\
\hline Facebook & $\begin{array}{l}\text { Accès à un grand nombre de participant(e)s poten- } \\
\text { tiel(le)s } \\
\text { Accès à une grande variété de participant(e)s poten- } \\
\text { tiel(le)s } \\
\text { Possibilité d'atteindre une population difficile d'accès } \\
\text { Contact rapide et direct (sans intermédiaire) } \\
\text { Processus familier } \\
\text { Possibilité pour les participant(e)s de réfléchir et de } \\
\text { poser des questions grâce aux contacts faits à distance } \\
\text { Réassurance et diminution des craintes associées au } \\
\text { contexte de la recherche } \\
\text { Contexte idéal pour les sujets sensibles et intimes } \\
\text { Respect de l'anonymat } \\
\text { Crédibilité renforcée lors de la création d'un compte } \\
\text { utilisé pour la recherche } \\
\text { Coût abordable }\end{array}$ & $\begin{array}{l}\text { Biais de recrutement } \\
\text { Risque de recruter des individus qui ne correspondent } \\
\text { pas aux critères d'inclusion } \\
\text { Identité plus difficile à vérifier } \\
\text { Risque de sous-représentation ou d'exclusion } \\
\text { Discrimination } \\
\text { Questionnement sur la crédibilité professionnelle en } \\
\text { utilisant un compte personnel } \\
\text { Design et contenu de qualité pour présenter le projet de } \\
\text { recherche } \\
\text { Mise à jours des publications nécessaires } \\
\text { Présence constante sur les réseaux sociaux ciblés } \\
\text { Expertise en informatique et en programmation néces- } \\
\text { saire }\end{array}$ \\
\hline $\begin{array}{l}\text { LinkedIn \& } \\
\text { ResearchGate }\end{array}$ & $\begin{array}{l}\text { Accès à une population plus difficile à recruter } \\
\text { Validation de l'identité des participant(e)s plus fiable } \\
\text { Contact rapide et facilité } \\
\text { Contexte professionnel }\end{array}$ & $\begin{array}{l}\text { Utilisation plutôt restreinte à l’Amérique du Nord } \\
\text { Performance médiocre pour le recrutement internatio- } \\
\text { nal } \\
\text { Langues variées } \\
\text { Limité aux membres }\end{array}$ \\
\hline
\end{tabular}


Figure 1 : Symptômes possibles de la Neurofibromatose de type $1^{\star \star}$ Peut varier entre les individus

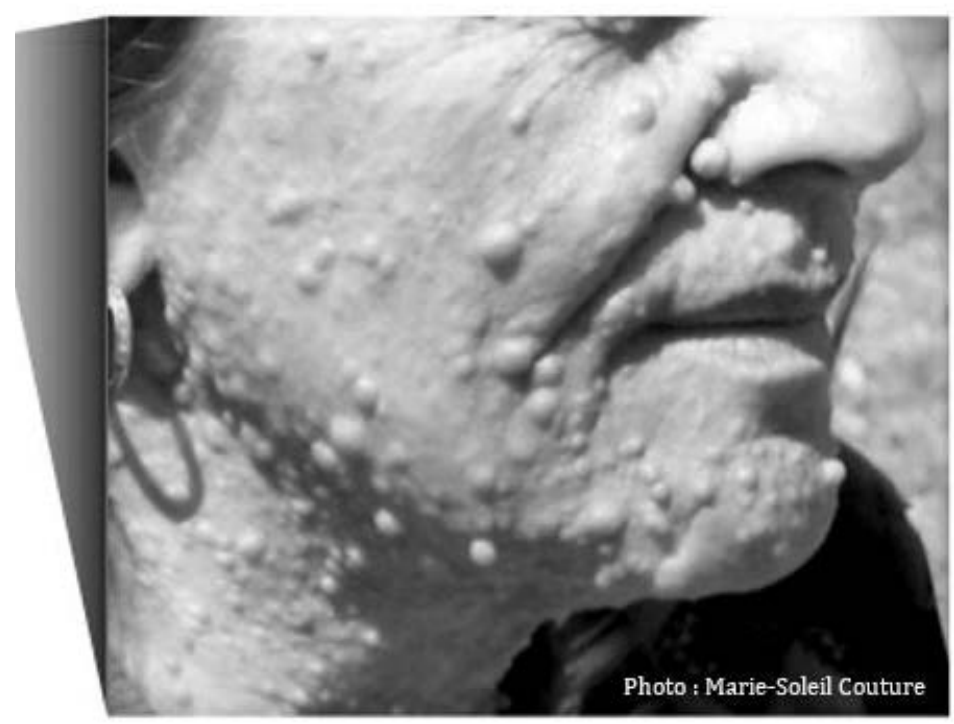

Neurofibromes
Taches café-au-lait sur la peau
Plexiformes
Nodules de Lisch
Taches de rousseur
Difformités osseuses
Difficultés d'apprentissage
Petite stature
Anomalies cardiaques
Gliomes optiques
Relations sociales difficiles
Effet sur le statut socioéconomique




\section{Bibliographie}

Anadón, M. (2006). La recherche dite «qualitative»: de la dynamique de son évolution aux acquis indéniables et aux questionnements présents. Recherches qualitatives. 26 (1), 5-31.

Barnes, S.B. (2004). Issues of attribution and identification in online social research. Online social research: Methods, issues, and ethics. 203-222.

Bouffard, C. (2017, mai). Pour une information et un consentement véritables. Conférence prononcée dans le cadre des Rencontres Hippocrate; Bordeaux, France. Repéré à https://www.leh.fr/evenement/rencontres-hippocrate

Bouffard, C., Duplain-Laferrière F., Noël V., Lapointe G. Bouffard G., Marin A. et Drouin R. (2017, septembre) Les CER et le recrutement en ligne, Présentation. $6^{\mathrm{e}}$ Colloque sur l'éthique de la recherche et l'intégrité scientifique en recherche sur la santé humaine, Montréal.

Bowen, G.A. (2008). Naturalistic inquiry and the saturation concept: a research note. Qualitative research. $8(1), 137-52$.

British Psychological Society. (2007). Report of the working party on conducting research on the Internet: guidelines for ethical practice in psychological research online. Repéré à http://www.bps.org.uk/sites/default/ files/documents/conducting_research_on_the_internet-guidelines_for_ethical_practice_in_psychological_research_online.pdf

Buchanan, E.A. et Hvizdak, E.E. (2009). Online survey tools: Ethical and methodological concerns of human research ethics committees. Journal of Empirical Research on Human Research Ethics. 4(2), 37-48.

Cardon, D. (2011). Réseaux sociaux de l'Internet. Communications. (1), 141-148.

Conseil de recherches en sciences humaines du Canada, Conseil de recherches en sciences naturelles et en genie, Instituts recherche santé Canada. (2014). Énoncé de politique des trois Conseils : Éthique de la recherche avec des êtres humains. (cité dans le texte comme EPTC2). Repéré à http://www.ger.ethique. gc.ca/pdf/fra/eptc2-2014/EPTC_2_FINALE_Web.pdf

Corbière, M. et Larivière, N. (dir.). (2014). Méthodes qualitatives, quantitatives et mixtes : dans la recherche en sciences humaines, sociales et de la santé. (1 ere éd.). Québec, Canada : Presses de l'Université du Québec.

Côté, J. (2012). Les enjeux éthiques de l'utilisation d'internet en recherche: principales questions et pistes de solutions. Revue internationale d'éthique sociétale et gouvernementale. 14 (2). Repéré à http://ethiquepublique.revues.org/997

Couper, M.P., Kapteyn, A., Schonlau, M. et Winter, J. (2007). Noncoverage and nonresponse in an Internet survey. Social Science Research. 36(1), 131-148.

Chardel, P.-A. et Reber, B. (2011). Risques éthiques. Communications. 88 (1), 149-157.

Chen, S.-L.S., Hall, G.J. et Johns, M.D. (2004). Research paparazzi in cyberspace: The voices of the researched. Online social research: Methods, issues, and ethics. 157-175.

Chu, J.L. et Snider, C.E. (2013). Use of a social networking web site for recruiting Canadian youth for medical research. Journal of Adolescent Health. 52(6), 792-794.

Ess, C. (2002). Ethical decision-making and Internet research: Recommendations from the AolR ethics working committee. Association of Internet Researchers (AolR). Repéré à http://www.aoir.org/reports/ethics.pdf 
Ess, C. (2006). «Ethics and the use of the internet in social science research». Dans A. Joinson, K. McKenna, T. Postmes et U. Reips (dir.). Oxford Handbook of Internet Psychology (p. 487-503). Oxford et New York: Oxford University Press.

Facebook. (2017). Company Info. Repéré à https://newsroom.fb.com/company-info/

Fielding, N.G., Lee, R.M. et Blank, G. (dir.). (2008). The Sage handbook of online research methods : Sage Publications.

Fortin, M. (2010). Fondements du processus de recherche: méthodes quantitatives et qualitatives. (2éd.). Montréal : Chenelière Éducation.

Frandsen, M., Walters, J. et Ferguson, S.G. (2013). Exploring the viability of using online social media advertising as a recruitment method for smoking cessation clinical trials. Nicotine \& tobacco research. 16(2), 247-51.

Frippiat, D. et Marquis, N. (2010). Web surveys in the social sciences: An overview. Population. 65 (2), 285-311.

Institut de la statistique du Québec, (2017, 25 juillet). Étude: Naissances, décès et accroissement naturel, Estrie et ensemble du Québec, 1991, 1996, 2001, 2006 et 2011-2016. Repéré à http://www.stat.gouv.qc.ca/statistiques/profils/profil05/societe/demographie/nais_deces/nais05.htm2.

Jean, É. (2015). Les enjeux liés à la collecte de données en ligne. La Revue des Sciences de Gestion. (2), 13-21.

Kosinski, M., Matz, S.C., Gosling, S.D., Popov, V. et Stillwell, D. (2015). Facebook as a research tool for the social sciences: Opportunities, challenges, ethical considerations, and practical guidelines. American Psychologist. 70(6), 543-556.

Kraut, R., Olson, J., Banaji, M., Bruckman, A., Cohen, J. et Couper, M. (2004). Psychological research online: report of Board of Scientific Affairs' Advisory Group on the Conduct of Research on the Internet. American psychologist. 59(2), 105.

Latzko-Toth, G., Proulx, S. (2013). «Enjeux éthiques de la recherche sur le Web», dans C. Barats (dir.), Manuel d'analyse du web en Sciences Humaines et Sociales, Paris: Armand Colin, p. 32-48.

Lenhart A., Duggan M., Perrin A., Stepler R., Rainie H. et Parker K. (2015). Teens, social media and technology overview 2015. Smartphones facilitate shifts in communication landscape for teens. Washington, DC: Pew Internet \& American Life Project.Linkedln. (2017).

Levinas, E. (1961). Totalité et In fi ni. Essai sur l'extériorité. La Haye, Pays-Bas : Martinus Nijhoff.

À propos de Linkedln. (2017). Repéré à https://www.linkedin.com/about-us?trk=hp-about

Luppi, M., et al., Chromosomally Integrated HHV-6, in Human Herpesviruses HHV-6A, HHV-6B \& HHV-7 (Third Edition) L. Flamand, et al., Editors. 2014, Elsevier: Boston. p. 251-265.

Mann, C. et Stewart, F. (2000) Internet communication and qualitative research: A handbook for researching online: Sage Publictions.

Mann, C. (2003). Generating data online: Ethical concerns and challenges for the C21 researcher. Applied ethics in internet research. 31-50.

McKee, H.A. et Porter, J.E. (dir.). (2009). The ethics of Internet research: A rhetorical, case-based process. New York, Etats-Unis : Peter Lang.Pastinelli, M. (2011). L'observation participante dans les démarches d'ethnographie en ligne. Communication présentée à l'École d'été sur les méthodes de recherche en ligne en santé de l'UQAM, Montréal, Canada. Repéré à http://comsante.uqam.ca/lobservation-participante-dans-les-demarches-dethnographie-en-ligne/ 
Odwazny, L.M. (2017). The "Nonexceptionalism" of Social Media Used for Subject Recruitment. The American Journal of Bioethics. 17(3), 17-19.

Pittenger, D.J. (2003). Internet research: An opportunity to revisit classic ethical problems in behavioral research. Ethics \& Behavior. 13(1), 45-60.

ResearchGate. (2017). About us. Repéré à https://www.researchgate.net/about

Ross, M.W., Månsson, S.-A., Daneback, K., Cooper, A. et Tikkanen, R. (2005). Biases in internet sexual health samples: comparison of an internet sexuality survey and a national sexual health survey in Sweden. Social Science \& Medicine. 61(1), 245-52.

Sveningsson, M. (2004). Ethics in Internet ethnography. Dans E.A. Buchanan (dir.), Readings in virtual research ethics: Issues and controversies (p. 45-61). Milwaukee, États-Unis : Information Science Publishing.

Teitcher, J.E., Bockting, W.O., Bauermeister, J.A., Hoefer, C.J., Miner, M.H., et Klitzman, R. L. (2015). Detecting, preventing, and responding to "fraudsters" in internet research: ethics and tradeoffs. The Journal of Law, Medicine \& Ethics, 43(1), 116-133

Thoër, C. (2011). L'entrevue en ligne : spécificités d'une méthode de plus en plus utilisée pour la recherche en santé. Repéré à http://comsante.uqam.ca/l\%E2\%80\%99entrevue-en-ligne-specificitesd\%E2\%80\%99une-methode-de-plus-en-plus-utilisee-pour-la-recherche-en-sante/

Ward, K.J. (1999). The Cyber-Ethnographic (Re) Construction of Two Feminist Online Communities. Sociological Research Online. 4(1). Repéré à http://socresonline.org.uk/4/1/ward.html

Waycott, J., Munteanu, C., Davis, H., Thieme, A., Moncur, W., McNaney, R., Vines, J. et Branham, S. (2016, May). Ethical encounters in human-computer interaction. In Proceedings of the $2016 \mathrm{CHI}$ Conference Extended Abstracts on Human Factors in Computing Systems (pp. 3387-3394). ACM.

Wilson, R.E., Gosling, S.D. et Graham, L.T. (2012). A review of Facebook research in the social sciences. Perspectives on psychological science. 7(3), 203-20.

Whitehead, L.C. (2007). Methodological and ethical issues in Internet-mediated research in the field of health: an integrated review of the literature. Social Science \& Medicine. 65(4), 782-791.

Zimmer, M. (2010). "But the data is already public": on the ethics of research in Facebook. Ethics and information technology. 12(4), 313-325. 
TITRE: CONCLUSION

Auteur(s): BÉAtrice Eysermann, Michel T. Giroux et Ana Marin

PublicATION: RECRUTEMENT ET CONSENTEMENT À LA RECHERCHE : RÉALITÉS ET DÉFIS ÉTHIQUES

PAGES: $203-209$

ISBN: 978-2-7622-0357-8

Directeurs: ANA MARIN, BÉATRICE EYSERMANN ET MICHEL T. GIROUX 


\section{Conclusion}

Béatrice Eysermann, Ph. D., coordonnatrice du Comité d'éthique de la recherche sectoriel en santé des populations et première ligne, CIUSSS de la Capitale-Nationale.

Michel T. Giroux, avocat et docteur en philosophie, directeur de l'Institut de consultation et de recherche en éthique et en droit (ICRED), président du Comité d'éthique de la recherche des établissements du Centre de recherche interdisciplinaire en réadaptation du Montréal métropolitain (CRIR), président du Comité d'éthique de la recherche Biobanque Genizon chez Génome Québec, professeur associé au département de médecine sociale et préventive à la Faculté de médecine de l'Université Laval.

Ana Marin, professeure associée Université de Sherbrooke, membre des Comités d'éthique de la recherche du CHU de QuébecUniversité Laval et du CISSS Chaudière-Appalaches, conseillère en éthique CISSS Chaudière-Appalaches, membre de l'Institut de consultation et de recherche en éthique et en droit (ICRED) et membre associée de l'IDEA.

\section{Discuter et conclure}

Nous avons traité, en colloque et dans les textes publiés dans cet ouvrage, de deux sujets qui vont de pair en recherche : le recrutement et le consentement. Cependant, il nous fallait à tout prix, pour nos deux colloques, éviter de tomber dans les méandres des processus d'approbation éthique. Ce que nous voulions, c'était que les conférenciers nous livrent leur expérience, leur vécu. Nous les avons prévenus dès le départ que le politiquement correct ne nous intéressait pas et que nous voulions entendre parler du concret, de ce qui se passe sur le terrain qu'ils fréquentent. En ce sens, nous n'avons pas été déçus! Et, de ces deux journées presque enflammées, nous avons retenu quatre idées phares que nous vous livrons en conclusion : l'impact du recrutement sur un consentement libre et éclairé, la véritable pertinence du formulaire dans le processus de consentement, l'incroyable créativité des chercheurs et des étudiants, et les balises nécessaires pour un dialogue de choix entre CER et chercheurs.

\section{Réflexions pour un recrutement volontaire et éclairé}

Tout d'abord, revenons sur quelques bases. Un des documents fondamentaux pour évaluer la validité éthique d'un projet de recherche au Canada est l'EPTC2. Le consentement à la participation étant le socle incontournable de la recherche depuis le Code de Nuremberg (1947), l'EPTC2 lui consacre un chapitre entier et explore plusieurs cas de figure. On y lit notamment un lien très étroit entre le recrutement des sujets et le caractère volontaire du consentement. Ainsi :

La manière d'envisager le recrutement constitue un élément important du consentement librement accordé. Quand, comment et où aborde-t-on les participants? Et qui les recrute? Voilà des questions qui représentent des éléments importants dans le renforcement ou l'affaiblissement du caractère volontaire du consentement (EPTC 2, $2014: 36)$.

Autrement dit, le processus de recrutement peut avoir un impact réel sur le caractère éclairé du consentement.

Faut-il que celui qui contribue de sa personne à la recherche soit appelé sujet ou participant? Un de nos auteurs explique pourquoi, contrairement à ce que propose l'EPTC2, le terme sujet devrait être privilégié. Le terme sujet, pris au sens philosophique de l'être autonome et conscient, est le plus susceptible d'inspirer au chercheur le 
respect à l'égard de celui qui contribue de sa personne à son projet de recherche. Évidemment, l'essentiel, c'est que la personne visée soit abordée et renseignée d’une manière respectueuse de sa dignité.

L'approche à privilégier tente d'éviter le phénomène de la méprise thérapeutique, particulièrement susceptible de se produire lorsque le chercheur est aussi le clinicien traitant du sujet approché. Nous avons aussi constaté que certains sujets semblent prêts à accepter n'importe quoi pour guérir de leur maladie, y compris lors des essais cliniques de phase I. Cette attitude est particulièrement observable chez les personnes qui se trouvent en proie au désespoir. En revanche, l'objectivité et la bienveillance fournissent une inspiration éthique au chercheur, elles bonifient sa pratique professionnelle, et leur exercice constitue une sauvegarde pour les sujets de recherche.

Pensons aussi à la protection de la confidentialité et au respect de la vie privée dès lors qu'on approche ceux qu'on a identifiés comme sujets potentiels. Pensons aux questions qui surgissent lorsqu'il s'agit de définir ou d'identifier les «marginaux» qui ne se pensent pas comme tels, et sur la manière, alors, de les recruter. Ou bien encore, pensons aux embûches qui se posent lorsqu'on recrute les membres d'une famille dans une recherche sur une maladie génétique héréditaire.

Nous posons alors cette notion que les processus de recrutement et de demande de consentement sont à la base d'une recherche éthique. Sous-jacent à cette notion, se trouve le respect de la personne ou, autrement dit, le respect et la protection de son autonomie, surtout lorsque celle-ci peut être mise à mal par des circonstances particulières liées à l'âge ou à l'état de santé (EPTC2, 2014 : 6-8). Nous avons simplement posé la question à nos conférenciers et auteurs de ce collectif : comment ce respect de la personne s'articule-t-il autour de la nature et du contexte spécifique de chaque projet de recherche? Comment ce respect se manifeste-t-il, considérant la vulnérabilité de la population à l'étude et le lien que le chercheur doit entretenir avec les sujets potentiels?

Et, au travers de ces colloques, nous tentions aussi de voir si les deux pans incontournables que sont le recrutement et le consentement pouvaient former un seul processus continu, qui est la clé de l'intégration d'un sujet dans une recherche autant qu'il permet le développement de la recherche. Peut-on imaginer alors parler d'un recrutement volontaire et éclairé, même si la formule pourrait surprendre?

Nos questionnements ont ainsi trouvé des réponses dans les présentations, puis dans les textes de nos auteurs.

\section{Faire du formulaire de consentement un véritable outil de pédagogie et de communication}

Le formulaire de consentement est une institution en éthique de la recherche. On peut toutefois se questionner sur l'accomplissement réel de sa raison d'être, soit d'éclairer le sujet pour lui permettre de consentir ou pas à la recherche de façon informée. La question de l'usage approprié du formulaire de consentement se pose à propos de tous les projets de recherche dont la mise en œuvre suppose l'existence d'un tel formulaire. Pour certains projets de recherche, nous pouvons nous demander à quoi le sujet consent vraiment, notamment lorsque cette personne souffre d'un trouble mental.

Mais, si le consentement est le maître-mot, nous pouvons aisément observer que, trop souvent, lors des séances d'un CER, parler du consentement revient à parler du formulaire de consentement, sans jamais en dépasser la forme ni se questionner sur son niveau de littératie ou celui des sujets potentiels à qui ce document s'adresse. Dans le processus d'obtention du consentement, tout sujet potentiel est entièrement dépendant de l'équipe de recherche. Ce facteur de dépendance devient plus déterminant lorsque le niveau de littératie de la personne 
ne lui permet pas de comprendre avec une relative facilité les explications qui lui sont données. Le principe de respect de l'autonomie impose à l'équipe de recherche de fournir des explications que la personne comprend.

Débattre d'un formulaire de consentement - lequel est considéré comme le document obligatoire pour obtenir/donner une approbation éthique - ressemble davantage à un combat de virgules et de formulations plutôt qu'à un questionnement concernant son véritable impact sur la compréhension du sujet visé. Et, même si c'est une critique qu'on entend constamment dans le monde de l'éthique de la recherche, de la part des chercheurs comme des CER, l'exercice de rhétorique perdure toujours entre les deux.

Pour sortir de cette ornière, une voie se dessine : sortir du document dans lequel on s'embourbe pour aller plus loin et demander au chercheur d'expliquer son processus de demande de consentement. Telle était précisément la raison d'être de notre colloque sur le consentement. Ce faisant, nous avons pu découvrir que nos auteurs ont su utiliser le formulaire de consentement pour en faire un véritable outil de communication et de pédagogie du chercheur envers le sujet. Auprès de sujets mineurs potentiels, nous avons constaté qu'un formulaire d'assentiment peut être transformé en un document explicatif qui rejoint les enfants, d'abord par son titre qui réfère à une permission plutôt qu'à un assentiment.

Notre premier apprentissage, nous en avons eu la preuve, fut celui-là : il est possible de discuter du consentement sans se limiter au formulaire de consentement. Tout au long du colloque, il fut davantage question du processus de demande de consentement que de l'obtention d'une signature à la fin d'un formulaire écrit. II nous semble encourageant, et nous voulons le souligner, de voir à quel point le respect des sujets, dans leur contexte et avec leur vulnérabilité particulière, est au cœur des préoccupations des chercheurs et de leurs équipes, au même titre que l'avancement des connaissances scientifiques. Si nous n'en doutions pas, nous avons été abreuvés d'illustrations des plus parlantes, et nous en remercions nos collaborateurs.

\section{La créativité au cœur des processus de recrutement et de consentement}

Le chercheur amorce toujours la préparation de sa recherche par un devis dans lequel on retrouve son approche théorique, sa méthodologie et sa connaissance initiale de l'objet de son étude. Mais, en réalité, c'est sur le terrain que tout va se jouer. C'est là, en effet, que surgissent les imprévus, les surprises et les défis avec lesquels il faut pouvoir composer, faute de quoi l'intégrité même de la recherche peut être compromise. Les chercheurs en sont témoins presque à chaque fois qu'ils passent de leur projet de recherche écrit à sa mise en œuvre, par le recrutement et le processus d'obtention du consentement.

Nos conférenciers puis nos auteurs nous ont conduits directement sur le terrain de leur créativité, celle qui les a fait sortir autant de leur zone de confort que de leurs habitudes méthodologiques. Cette créativité s'est alors exprimée autant dans le processus de recrutement que dans celui du consentement. Ce collectif en recèle plusieurs exemples dignes d'être notés. Pensons notamment au recrutement des adolescents atteints de neurofibromatose 1 , un recrutement davantage désiré par les parents que par leur enfant ou bien aux trésors d'ingéniosité nécessaires au recrutement de patients atteints de traumatismes craniocérébraux et de la maladie d'Alzheimer.

Il en ressort ce constat imparable: dans tous les exemples qui composent ce collectif, la créativité des chercheurs et de leur équipe s'est exprimée à travers leur connaissance fine du terrain et des contextes, une connaissance qui s'est aussi approfondie et affinée directement sur le terrain. Certains de nos auteurs exposent comment cette créativité va de pair avec un respect accru pour les sujets à la recherche. 
Et l'apprentissage le plus fondamental est sans doute celui de la nécessité d'investir du temps, de prévoir davantage de temps pour le recrutement et pour obtenir le consentement. II s'agit de voir venir, d'éviter de se laisser surprendre, de savoir comprendre et s'adapter aux réalités du terrain de la recherche (effectuer un rappel téléphonique, se tourner vers les réseaux sociaux). Ainsi, les stratégies de recrutement de sujets autochtones dans trois communautés furent largement axées sur le lien de confiance tissé dans la durée.

\section{Poursuivre le dialogue}

Quelques années après l'invitation du MSSS au dialogue et à la confiance entre chercheurs et CER (2010), on constate qu'il reste du travail à faire, comme nous le rappellent plusieurs des auteurs de ce collectif, chercheurs et membres de CER. Les acteurs de la recherche, chercheurs et équipes, ainsi que les CER caressent les mêmes buts : l'avancement des connaissances et la protection des sujets à la recherche. Les auteurs nous invitent par contre à un juste milieu, à une approche dans laquelle réside la vertu, si on fait référence à l'éthique aristotélienne. Face à une attitude de protection parfois exagérée, qu'il s'agisse de celle des CER ou des tiers impliqués dans le recrutement, les auteurs nous invitent, à partir de leur expérience, à leur faire davantage confiance.

À partir de ce dernier constat (le besoin de poursuivre le dialogue), nous ouvrons une porte tant pour les chercheurs que pour leur CER. Le champ d'action des CER ne saurait se limiter au seul contenu des protocoles. Les chercheurs ont la responsabilité de montrer, décrire, expliquer, révéler même, leur connaissance profonde du terrain et des contextes - responsabilité qui est trop peu souvent exercée. L'idée est de faire un travail de collaboration qui appelle à l'ouverture, tant de la part des CER que des chercheurs, pour saisir en quoi, par exemple, la créativité est parfois incontournable pour mener à bien un projet.

Ainsi, à la lumière de ce que nous avons appris de nos conférenciers et de nos auteurs, nous ne pouvons conclure qu'à la nécessité d'ajuster les recherches en fonction du contexte dans lequel elles prendront corps, c'est-à-dire « chez » les sujets eux-mêmes. II faut donc que la recherche puisse s'ajuster au contexte, et non l'inverse. C'est pourquoi il nous faut insister sur le fait qu'une conversation éthique est toujours nécessaire entre les deux parties agissantes en éthique de la recherche, CER comme chercheurs. Si les CER peuvent nourrir la réflexion éthique des chercheurs de par leur expertise en éthique, les chercheurs peuvent réciproquement nourrir la réflexion des CER sur les nouvelles questions qui se posent dans la réalité de leur recherche. La confiance et l'ouverture de part et d'autre sont incontournables pour que ce dialogue ait lieu, mais cela demande de l'humilité. Mais aussi autre chose...

Car, si nous voulons que les chercheurs remportent ce défi, il faut pouvoir leur donner voix au chapitre, c'est-àdire leur donner tant l'espace de réfléchir que de publier. Nos colloques ont su nous démontrer que cet espace est absent, mais tellement nécessaire. Si, lors des colloques, les chercheurs et les étudiants ont su partager sans fard leurs préoccupations, leurs défis, leurs écueils aussi, l'interaction dans la salle a permis des débats profonds, quel que soit le domaine de recherche dans lequel on exerce. Les recherches cliniques et expérimentales et celles en sciences sociales ont partagé un même espace de discussion en se répondant les unes aux autres sur les enjeux du recrutement et du consentement. Les deux sphères se retrouvaient dans cet espace de discussion commun, permettant ainsi de découvrir que les difficultés étaient souvent semblables, parfois les mêmes, les stratégies applicables parfois transposables. Ce fut toute la richesse de nos journées. 
Que nous disent les publications dans le secteur de la recherche? Rares sont celles qui nous parlent de la méthodologie ou de l'expérience du terrain. Les publications, et c'est aussi leur raison d'être, se concentrent avant tout sur les résultats de la recherche, et non sur l'expérience du terrain en tant que telle - exception faite peut-être des anthropologues qui en ont fait leur science. Si on trouve des publications qui se penchent surtout sur l'expérience terrain, c'est parce que ledit terrain a soulevé de nombreux enjeux que les chercheurs ont voulu mettre en lumière afin de parler de leur expérience. Pourtant, il y a là toute une richesse qui mérite d'être divulguée et qui représente un matériau de choix pour les chercheurs et étudiants de toutes les disciplines dès lors qu'une participation humaine doit être sollicitée. C'est ce que nous avons voulu faire dans ce collectif, c'est-à-dire garder une trace de ces expériences de terrain dont nous avons beaucoup appris.

Puisque l'éthique de la recherche n'appartient pas aux CER ni aux conseillers en éthique, pas plus qu'aux chercheurs eux-mêmes, un espace de discussion et de partage est nécessaire pour nous enrichir réciproquement de nos réussites et de nos échecs dans le processus de recrutement et de consentement. Humblement, nous espérons y avoir contribué en organisant les deux colloques et en réalisant cette publication.

Béatrice, Michel et Ana 


\section{Bibliographie}

Conseil de recherches en sciences humaines du Canada, Conseil de recherches en sciences naturelles et en génie du Canada, Instituts de recherche en santé du Canada (décembre 2014). Énoncé de politique des trois Conseils : Éthique de la recherche avec des êtres humains. (cité dans le texte comme EPTC2) http://www.ger.ethique.gc.ca/pdf/fra/eptc2-2014/EPTC_2_FINALE_Web.pdf

Thoër, C., Millerand, F., Myles, D., Orange, V. et Gignanc O. (2012). Enjeux éthiques de la recherche sur les forums Internet portant sur l'utilisation des médicaments à des fins non médicales. Communiquer, 7. https://journals.openedition.org/communiquer/1085 